\title{
Willingness to pay for physician services in Ukraine and other central and eastern European countries : application of stated preferences techniques to the assessment of patient charges
}

Citation for published version (APA):

Danyliv, A. (2014). Willingness to pay for physician services in Ukraine and other central and eastern European countries : application of stated preferences techniques to the assessment of patient charges. [Doctoral Thesis, Maastricht University]. Maastricht University. https://doi.org/10.26481/dis.20140226ad

Document status and date:

Published: 01/01/2014

DOI:

10.26481/dis.20140226ad

Document Version:

Publisher's PDF, also known as Version of record

Please check the document version of this publication:

- A submitted manuscript is the version of the article upon submission and before peer-review. There can be important differences between the submitted version and the official published version of record. People interested in the research are advised to contact the author for the final version of the publication, or visit the DOI to the publisher's website.

- The final author version and the galley proof are versions of the publication after peer review.

- The final published version features the final layout of the paper including the volume, issue and page numbers.

Link to publication

\footnotetext{
General rights rights.

- You may freely distribute the URL identifying the publication in the public portal. please follow below link for the End User Agreement:

www.umlib.nl/taverne-license

Take down policy

If you believe that this document breaches copyright please contact us at:

repository@maastrichtuniversity.nl

providing details and we will investigate your claim.
}

Copyright and moral rights for the publications made accessible in the public portal are retained by the authors and/or other copyright owners and it is a condition of accessing publications that users recognise and abide by the legal requirements associated with these

- Users may download and print one copy of any publication from the public portal for the purpose of private study or research.

- You may not further distribute the material or use it for any profit-making activity or commercial gain

If the publication is distributed under the terms of Article 25fa of the Dutch Copyright Act, indicated by the "Taverne" license above,

Download date: 26 Apr. 2023 


\section{WILLINGNESS TO PAY FOR PHYSICIAN SERVICES IN UKRAINE AND OTHER CENTRAL AND EASTERN EUROPEAN COUNTRIES:}

APPLICATION OF STATED PREFERENCES TECHNIQUES TO THE ASSESSMENT OF PATIENT CHARGES

Andriy Danyliv 
Willingness to pay for physician services in Ukraine and other Central and Eastern European countries: Application of the stated preferences techniques to the assessment of patient charges.

(C) Andriy Danyliv, 2014

All rights reserved. No part of this publication may be reproduced, stored in a retrieval system, or transmitted in any form or by any means, electronic, mechanical, photocopying, recording or otherwise, without the written permission from the author.

ISBN: 978-90-821059-1-9

Cover design by Bohdana Vinogradova

Printed by Dom Reklamy LLC, Kharkiv, Ukaine 


\title{
WILLINGNESS TO PAY FOR PHYSICIAN SERVICES IN UKRAINE AND OTHER CENTRAL AND EASTERN EUROPEAN COUNTRIES:
}

\author{
APPLICATION OF STATED PREFERENCES TECHNIQUES \\ TO THE ASSESSMENT OF PATIENT CHARGES
}

\author{
DISSERTATION \\ to obtain the degree of Doctor at Maastricht University, \\ on the authority of the Rector Magnificus, Prof. dr. L. L. G. Soete \\ in accordance with the decision of the Board of the Deans, \\ to be defended in public \\ on Wednesday 26 February 2014, at 10:00 hours
}

by

Andriy Danyliv 


\section{Supervisor:}

Prof. dr. W.N.J. Groot

\section{Co-supervisors:}

Dr. M. Pavlova

Dr. I. Gryga (National University of Kyiv-Mohyla Academy)

\section{Assessment Committee:}

Prof. dr. S.M.A.A. Evers (chair)

Prof. dr. I. Burakovsky (National University of Kyiv-Mohyla Academy)

Prof. dr. C.D. Dirksen

Prof. dr. G.G. van Merode

Dr. M. Wynnyckyj (National University of Kyiv-Mohyla Academy)

\section{Acknowledgement of funding:}

The study is financed by the European Commission under the 7th Framework Program, Theme 8 Socio-economic Sciences and Humanities, Project ASSPRO CEE 2007 (Grant Agreement no. 217431). The views expressed in this dissertation are the sole responsibility of the author and do not necessarily reflect the views of the European Commission or its services. 


\section{Contents}

$\begin{array}{lll}\text { Chapter } 1 & \text { General introduction and background } & 7\end{array}$

Chapter 2 Preferences for physician services in Ukraine: A discrete 41 choice experiment

Chapter 3 Willingness to pay for physician services at a primary

63 contact in Ukraine: Results of a contingent valuation study

Chapter 4 Willingness to pay for physician services: Comparing 81 estimates from a discrete choice experiment and contingent valuation

Chapter 5 Willingness and ability to pay for physician services in six 97 Central and Eastern European countries

Chapter 6 Calibration of the willingness to pay for physician services in six Central and Eastern European countries: Context implications for the over- and understatement patterns.

Chapter 7 General discussion

References

Appendices

Appendix A Brief methodological report on data collection: Small scale (pilot) survey in Ukraine in 2009

Appendix B Brief methodological report on data collection: Survey from 182 six CEE countries in 2010

Appendix C Examples of the valuation tasks used in the small scale survey in Ukraine in 2009

Appendix D An example of a CV task used in the full scale survey in six 
Summary

Samenvatting

Анотація 205

Аннотация

211

Thank you words

Curriculum Vitae 219 
CHAPTER 1

General introduction and background 


\subsection{The scope of the dissertation}

In Europe, patient cost-sharing (patient charges for goods and services in the public health care system) is common for pharmaceuticals and medical devices. For other health care services, it is less common, especially in the Central and Eastern European (CEE) countries. The absence of patient cost-sharing for services in most of these countries is primarily caused by a lack of political will and ability to introduce such charges rather than based on evidence on their positive or negative effects (Tambor et al., 2011). This dissertation focuses on the assessment of patient charges for services in the public health care systems in CEE countries. In particular, the focus is on the charges for primary outpatient services, i.e. visits to a physician, including GP and medical specialist.

Designing and implementing patient cost-sharing is a complex process in any country irrespective of the level of economic development. The complexity consists in the trade-off between their effects on efficiency and equity in health care provision. The former are rather uncertain while the latter are overall negative (Sepehri and Chernomas, 2001).

As a policy tool, official patient charges for services and goods in the basic package have two main objectives: reducing excessive use of health care resources (ex-ante and ex-post moral hazard) and raising additional funds for the health care system (Drummond and Towse, 2012; Pavlova et al., 2012). At a system level, patient cost-sharing is found to have a positive impact on overall efficiency in OECD countries (Wranik, 2012). However, it has a relatively low ability to generate additional funds due to the high collection costs (Pavlova et al., 2010).

Earlier studies exploiting data from natural experiments, such as the RAND experiment held in the 1970s in the USA, suggested a very wide range of price elasticities of health care consumption (see Zweifel and Manning, 2000). Studies from Ireland (Nolan, 1993), France (Chiappori et al., 1998), Belgium (Van de Voorde at al., 2001; Cockx and Brasseur, 2003; Carrin and Van Dael, 1990; Van Doorslaer, 1984), Germany (Winkelmann, 2004; Schmitt et al., 2009; Rückert et al., 2008), and Taiwan (Chen et al., 2009) report different consumer reactions to the introduction or increase in co-payments for in- and outpatient care. Most of them find a payment increase or introduction to have a significant negative impact on service use, though opposite findings exist as well (Chiappori et al., 1998; Augurzky et al., 2006).

Another important policy objective of official patient charges for services and goods in the basic package is regulating the market, in particular dealing with informal patient payments to health care providers (Lewis, 2007; Ensor and Thompson, 2011; Gaál et al., 2010). Informal 
payments are widely spread in most CEE countries (Balabanova and McKee, 2002; Belli et al., 2004; Ensor, 2004; Gaál et al., 2010; Gaál and McKee, 2005; Szende and Culyer, 2006; Thompson and Witter, 2000). Kyrgyzstan and Cambodia are often presented as successful example of formalizing informal payments (Jakab and Kutzin, 2009; Baschieri and Falkingham, 2006; Barber et al., 2004; Akashi, 2004). But overall it is recognized that official charges cannot eradicate informal payments on their own and should be applied together with other measures (Stepurko et al., 2013).

Despite these potentially beneficial features, patient charges for services and goods in the public health care system may have adverse effects in terms of prohibiting or reducing necessary health care use for those who cannot afford to pay, impairing access and equity, and causing a double financial burden to patients in case of persistent informal payments (Aarva et al., 2009; Baji et al., 2012b; Ensor, 2004; Lewis, 2000; Thompson and Witter, 2000; Sepehri and Chernomas, 2001). Also, some studies show formal patient payments to be regressive and to have significant impoverishing effects on household wellbeing (e.g. Baji et al., 2012a; Rückert et al., 2008; Ching, 1995; Wagstaff and van Doorslaer, 1992).

The evidence about the effects of patient cost-sharing mentioned above mostly comes from the USA, Western Europe and developed countries in Africa and Asia. Virtually all these studies are based on actual expenditures or past choices of consumers in real markets, which is called the revealed preferences approach (RP). However, it is difficult to use such data for the analysis of the effects of patient charges for basic health care services in CEE countries since most of these countries provide these services officially free-of-charge. Thus, there are no data about consumer behavior under different pricing regimes. In this case, the stated preferences approach (SP) might provide the necessary information. This implies eliciting values that people attach to certain health care services, or more broadly health benefits, by asking them to state these values, e.g. in terms of willingness to pay (WTP) for the benefits, or asking them to make hypothetical choices across benefit profiles.

Although SP have found a broad application in the assessment of health care programs and benefits, their application to policy analysis, and specifically the analysis of patient payment policies, is rare. The main problem with SP is their hypothetical nature and uncertain predictive validity (Groot et al., 2006; Murphy et al., 2005). Policy-makers, who are unfamiliar with SP, may find it difficult to adopt such data in the policy-making process. Therefore, this dissertation focuses on the application of the SP techniques to the assessment of patient charges for outpatient physician services, taking the CEE countries as a case study. 


\subsection{Background}

In the last years, SP approaches are increasingly used to value benefits in health care including health outcomes and health care services (Bridges et al., 2011; Smith and Sach, 2010). This is however, accompanied by a debate about the validity of the obtained results and the possibility to make policy and allocation decisions based on them. In this section, we review the most recent applications of SP in health care in order to demonstrate in which manner these methods are usually applied, and which design and methodological aspects make them applicable to policy decisions. This also justifies the methodological choices made in this dissertation and outlines gaps in research, which motivate this dissertation.

\subsubsection{Stated preference techniques}

The two most applied SP techniques are discrete choice experiments (DCE) and the contingent valuation technique (CV). The main difference between these two techniques is the way the values are obtained. In CV, a benefit is typically valued in a holistic manner either by open- or close-ended questions. In the former case, respondents are asked to state their willingness to pay (WTP) an amount for obtaining that benefit or willingness to accept (WTA) an amount for the loss of the benefit. In the latter case, WTP or WTA is assessed in the form of yes-no or double-bound question(s), or a bidding game. The obtained values are integral (sometimes referred to as discrete), i.e. they represent a mean value for a unit of the benefit (Mogas et al., 2009). In DCE, in contrast, the value of the benefit is described through a set of its characteristics (attributes of a profile), their impact is evaluated jointly, and the decomposed effects of the attributes on the value of the benefit are obtained. Thus, the retrieved values are marginal in nature and represent the relative weight of the characteristics in the value of the benefit. The marginal rate of substitution with the price attribute, consequently, represents the marginal WTP level for a small improvement in the non-price attributes under consideration (Mogas et al., 2009). Both DCE and CV are applied in this dissertation to study WTP for physician services.

Theoretically, choice-based elicitation methods are considered to be superior to non-choicebased methods as the former better resemble the real market (Zweifel et al., 2006; Louviere et al., 2000). In earlier applications, conjoint analysis in the form of rating and ranking of the presented profiles has been applied. However, nowadays these forms are considered to be outdated and DCE (choice-based method) is overall applied. In CV applications, however, the variety of both close-ended choice-based and open non-choice-based formats still remain, 
though a general shift towards choice-based elicitation formats is observed (Smith and Sach, 2010; Klose, 1999; Diener et al., 1998).

Such enthusiasm about choice-based techniques is, however, not supported by the results of the empirical literature. Typically, WTP in a real market (actual money contributions) is considered as a benchmark for exploring the theoretical validity of WTP obtained in SP. Evidence from inside and outside health care suggests that open-ended format of SP yields WTP that is either higher (Blumenschein et al., 2001) or close to WTP in real market choices (Ryan and Watson, 2009). The choice-based format, in turn, including both DCE and CV, usually yields WTP, which is substantially higher than that obtained in open-ended elicitation formats (e.g. Whitty et al., 2013; Loh and Shapiro, 2013; Danyliv et al., 2012a). Thus, WTP from the choice based format is even further from the real WTP than the one from the openended formats. In any case, WTP obtained via SP is to a large extent affected by the design of the survey and the empirical strategies used to model preferences.

The main motivation to use SP is their ability to provide monetary values for previously unknown and non-market traded benefits. Apart from this, the actual price people pay in a real market may not represent the maximum price they would be willing to pay and thus, estimates might be far from optimal. SP, in contrast, may provide a good estimate for the reservation price. SP, in addition allow capturing passive use values, i.e. utility obtained from the mere availability of a benefit without usage. This feature is especially important in primary health care (the application area of this dissertation) where every citizen is a potential user. All these advantages lead to an increased use of SP for valuing health benefits (for reviews see Ryan et al., 2001b; Smith and Sach, 2010; Klose, 1999; Diener et al., 1998).

The problems of SP are caused by their hypothetical nature. In case of the RP approach, values are based on real consumer behavior. SP values are based on hypothetical scenarios which respondents might not be familiar with and do not require real monetary commitments form the respondents or respective behavior in future. Moreover, it is often cognitively difficult and respondents may not fully understand the specification of the benefit or risks involved. All this leads to the hypothetical bias, i.e. respondents might state the values which are not true and are different form their WTP that would occur in real market decisions. Moreover, respondents might behave strategically and give on purpose answers which are not true but correspond to some desirable patterns. For example, if they believe that high stated values increase the probability of the benefit provision at zero prices, they might overstate their WTP. On the other hand, if it seems likely that the stated price has payment policy 


\section{Chapter 1}

consequences, respondents might understate the true value. All these problems might be addressed to certain extent through the correct design of the hypothetical tasks and/or appropriate data analysis and interpretation of the obtained results.

\subsubsection{Willingness and ability to pay}

In economics, the value of a benefit is assessed through one of two competing concepts: WTP or WTA, which basically seek to obtain the reservation price of a benefit. WTP is defined as the maximum amount that an individual would accept to pay in order to obtain a benefit or avoid loss. WTA, in contrast, is the minimal compensation an individual would accept for a loss of a benefit or acquiring something undesirable. It is well recognized, that WTA estimates are in general higher than WTP estimates (Whynes and Sach, 2007). There are several possible effects explaining this discrepancy and they might apply simultaneously. The discrepancy might occur due to the fact that WTA, unlike WTP, is not constrained by the individual's budget (Brown and Gregory, 1999). Endowment effect or loss aversion explains this effect through the idea that people value more that they are entitled to than something that they can probably acquire (Thaler, 1980). Finally, losing a benefit requires its substitution, and the lack of substitutes might increase its valuation (Hanemann, 1991).

In case of measuring ordinal preferences, i.e. mapping the relative desirability of the benefits or their characteristics, the discrepancy between WTA and WTP does not make much difference and both measures might provide consistent results. However, the preferences measured in cardinal monetary terms have meaning only with respect to a budget constraint. Hence, in this case application of WTA is theoretically inconsistent. Therefore, WTA is very rarely applied in health care for valuing benefits or losses with the purpose of investment decisions. In most cases when WTA is applied, it is to obtain providers' values (e.g. Wang and Hong, 2012; Rischatsch and Zweifel, 2012) or for the purpose of comparison with WTP (e.g. Martin-Fernandez et al., 2010a). NOAA recommends the use of WTP measures for the valuation of environmental amenities with the motivation that it yields more conservative estimates (Arrow and Solow, 1993). In this dissertation, we use the WTP concept as a more theoretically consistent approach, especially because we focus on the pricing effects.

The assumption that WTP, representing a payment, is anchored on a budget constraint (in contrast to WTA) might be erroneous in its turn. Russell (1996) argued that many policy and research debates tend to rely only upon WTP assuming that it is synonymous with ability to pay, which is not true in practice. WTP, whether stated or observed in actual purchase 
behavior, does not necessarily represent affordable purchase decisions. The households may apply resource mobilizing strategies, such as selling assets, sacrificing other needs, or borrowing money (Russell, 1996). Such decisions might be unaffordable in principle as they lead to long-term adverse effects on household welfare.

Actual health expenditures in many developing countries, and in CEE in particular, are shown to have impoverishing effects causing substantial assets depletion (e.g. Arsenijevic et al., 2012; Tomini et al., 2012b; Bredenkamp et al., 2011; Liu et al., 2011; van Doorslaer et al., 2006; Damme et al., 2004; Xu et al., 2003). Thus, they should not be regarded as affordable. Moreover, individuals faced with serious economic problems and at the same time with the necessity of substantial health expenditures, may change the structure of their preferences towards health care rather than other consumption categories (Mataria et al., 2006). They might even be unable to formulate their preferences definitely (Sen, 1992). Thus, their expressed WTP and amounts they actually pay for health care services might substantially exceed what would be affordable to them under normal conditions (without adverse long-term welfare effects). Therefore, in the valuation studies, especially those informing policy decisions about patient payment policies, it is crucial to account for the ability to pay.

Separate estimates of the ability to pay and WTP, however, provide little information for policy decisions. In economics, the well-known willingness and ability to pay (WATP) concept represents the demand and, therefore, provides the needed information about consumer reactions to the price changes. In many studies, actual expenditure is assumed to represent demand, i.e. WATP, for health care services (Ching, 1995; Gertler and van der Gaag, 1990; Mocan et al., 2004). However, actual expenditure is a poor representation of the true WATP which might be higher or as discussed above even lower than the amounts spent (Russell, 1996). Some studies use the opportunity cost of the undertaken purchase decisions (e.g. Saulo et al., 2008) as an indication of ability to pay, but not WATP.

In this dissertation, we focus on eliciting WATP rather than WTP. In the SP framework, the discrepancy between WTP and ability to pay might be exacerbated by the hypothetical bias as the respondents do not consider their real budget constraints when making hypothetical choices (Loomis et al., 1994). Thus, for the purpose of eliciting WATP in CV tasks of this study it is specified that the respondent should consider his/her own conditions and express what s/he would be willing and able to pay for the valued service. In DCE, incorporation of the ability to pay is not straightforward due to the choice-based elicitation format. In this dissertation, we simply assure that the respondent considers his/her own budget by asking one 


\section{Chapter 1}

to consider one's own real conditions before making the hypothetical choice. Due to the common practice of using WTP concept, we further refer to WATP when talking about CV results only. For the DCE results and for general use, e.g. referring to its use in the literature, we still use the term WTP.

\subsubsection{Integral and marginal WTP, and study perspective}

Most of the current SP literature in health care adheres to the perspective of an investment or health care provision agenda, or providing specific information for cost-benefit analysis. That is these studies seek to quantify benefits that would be gained from a provision of a certain service or from the improvements in the quality or access characteristics of these services (e.g. Regier et al., 2012; Naik-Panvelkar et al., 2012; Kjaer et al., 2012; Glenngard et al., 2012; Gidengil et al., 2012; Corso et al., 2013; Brandt et al., 2012). Some studies, however, are rather cautious even in interpreting the WTP estimates as benefit gains and simply use them as an indication of relative preferences (e.g. Whitty et al., 2013; Sadique et al., 2013; Carroll et al., 2013; Hancock-Howard et al., 2012; Ethier et al., 2012; Dixon et al., 2012). That is they answer questions like which characteristics are most important for the patients/consumers, what is the trade-off between the characteristics, and which of the improvements may facilitate consumption. On the other hand, many studies do consider WTP obtained as an indication of the value of the real benefit and discuss possibilities of the service/benefit provision by comparing gains to its cost (e.g. Shafie \& Hassali, 2013; Loh \& Shapiro, 2013; Lang et al., 2012; Kjaer et al., 2012; Glenngard et al., 2012; Asgary, 2012) or define this WTP as an acceptable cost range (e.g. Regier et al., 2012; Naik-Panvelkar et al., 2012).

WTP yielded from SP techniques, however, can also provide information to quantify the potential consumption effects of the price arrangements, or more generally, estimating demand. Such perspective represents the specific interest of this dissertation. As discussed in section 1.2.2., it implies the stronger assumptions that stated WTP (in this case WATP) well reflects the price that people would actually be willing and able to pay in the real market. Therefore, this perspective is very rare in health care. Some papers adopt this perspective, but straight away state that these estimates might be subject to a hypothetical or strategic bias (e.g. Mattsson et al., 2013; Lynn et al., 2013). Other studies call for the development of a special framework for the payment design that incorporates also actual consumption data (e.g. Naik-Panvelkar et al., 2012; Marti, 2012). Very few studies so far have fully relied upon SP 
for estimating demand and/or discuss pricing effects (e.g. Sadique et al., 2013; Mataria et al, 2007; Ho et al., 2005; Cropper et al., 2004).

The perspective of the study determines the choice of the SP eliciting technique (DCE or CV). Given the nature of the values estimated, DCE best suits the purpose of eliciting and quantifying relative preferences for benefit attributes, while CV is best used for obtaining the holistic value of the service, good, or other benefit. However, the correspondence is not always straightforward.

Most applications of CV use holistic evaluation principles (e.g. Whitty et al., 2013; Shafie and Hassali, 2013; Loh and Shapiro, 2013; Hansen et al., 2013, Corso et al., 2013, Wang and Hong, 2012; Sogaard et al., 2012; Lang et al., 2012; Havet et al., 2012; Gyrd-Hansen and Kjaer, 2012; Ethier et al., 2012; Dixon et al., 2012; Brandt et al., 2012; Bobinac et al., 2012b; Asgray, 2012). This leads to the idea, that the choice of CV technique is driven by the aim of obtaining holistic values. However, there are applications where CV is also used for valuing certain attributes of health care services. In this case, WTP is elicited for a certain characteristic separately or a decomposed scenario is applied (e.g. Mataria et al., 2007; Hammerschmidt et al., 2004; O’Brien and Gafni, 1996). In the former case, there is a loss in the theoretical validity of the results as the consumers are believed to make choices based on a whole set of relevant characteristics, not a single one. The decomposed scenario approach produces so-called sub-additive values that cannot be simply added to obtain the holistic value, and an aggregation function is offered as a solution to this (Hammerschmidt et al., 2004).

DCE produces marginal WTP values, which allows for defining relative preferences across the set of characteristics relevant for the choice. Hence, the primary purpose of DCE should be to define relative preferences, i.e. the main choice or value drivers. This application is observed in a substantial share of current studies (e.g. Sadique et al., 2013; Prosser et al., 2013; Moia et al., 2013; Naik-Panvelkar et al., 2012; Hodgkins et al., 2012; Gidengil et al., 2012). Nonetheless, nowadays, most DCE studies aim at deriving the integral value for a valued benefit.

A simple approach to obtain the integral value is when the benefit of interest is embedded into the DCE tasks as one of the attributes of a more complex service (e.g. Hancock-Howard et al., 2012). The majority of the DCE studies, however, apply integration of marginal WTP across the changes in the attributes, which are deemed to represent the benefit (Whitty et al., 2013; Mattsson et al., 2013; Carroll et al., 2013; Regier et al., 2012; Marti, 2012; Kjaer et al., 2012; 


\section{Chapter 1}

Jendle et al., 2012; Hancock-Howard et al., 2012; Glenngard et al., 2012). This approach is however not always possible to apply as it requires comparison of a valued benefit with certain attributes to a meaningful alternative with its own level of attributes. Some researchers design such goods/services (e.g. Mattsson et al., 2013; Regier et al., 2012) or use evidence on the characteristics of available alternatives (e.g. Jendle et al., 2012). Others use the total variation change in all the attributes (e.g. Glenngard et al., 2012) or calculate a change from the 'worst' to 'best' profiles of a benefit (e.g. Carroll et al., 2013; Lancsar and Savage, 2004). In any case, integrating marginal WTP across the changes in the attributes of the benefit is subject to a scale problem (Mogas et al., 2009). In essence, this means that the marginal WTP values are true only for very small changes in attribute levels from those that are common to a respondent. Integrating across large discrete changes in attribute levels imposes a restrictive assumption that the marginal WTP is constant across the whole range of an attribute, which is erroneous from an economics perspective. In addition, this approach is criticized for not accounting for the uncertainty inherent to the choices (Lancsar and Savage, 2004). Lancsar and Savage (2004) offered to use Hicksian variation measures instead, which implies predicting latent utility functions with and without a benefit and weighting their difference on the marginal utility of income (Hicks, 1943). This approach has been very rarely applied in health care (e.g. Kjaer et al., 2012). For small changes in attributes, it is argued to reduce to the integration of marginal WTP (Ryan, 2004b; Santos Silva, 2004).

In this dissertation, we keep to the point of view that CV is best for holistic WTP/WATP measures, while DCE only provides marginal WTP estimates. We also treat marginal WTP as the representation of the relative importance of the valued service attributes which cannot be integrated to obtain holistic values. Therefore, for the purpose of studying payment effects, $\mathrm{CV}$ is applied. DCE values are discussed from the perspective of priority setting, i.e. for ranking investment options related to quality and access improvements.

\subsubsection{Health benefits}

SP studies are applied in a vast variety of clinical fields with the most popular field being infectious diseases (e.g. Sadique et al., 2013; Prosser et al., 2013; Hansen et al., 2013; Regier et al., 2012; Gidengil et al., 2012; Asgray, 2012). Other popular fields are cardiovascular diseases (e.g. Whitty et al., 2013; Moia et al., 2013; Sogaard et al., 2012), cancer (e.g. Lang et al., 2012; Havet et al., 2012; Ethier et al., 2012), women's reproductive health (e.g. Mattsson

et al., 2013; Lynn et al., 2013), mental health (e.g. Hancock-Howard et al., 2012; Glenngard 
et al., 2012), asthma (e.g. Naik-Panvelkar et al., 2012; Brandt et al., 2012), digestive system disorders (e.g. Hodgkins et al., 2012; Dixon et al., 2012). Other clinical fields of SP application include genetic disorders (e.g. Carroll et al., 2013), nephrology (e.g. Kjaer et al., 2012), glucose related problems (e.g. Jendle et al., 2012), smoking cessation (e.g. Marti, 2012). The list is however not exhaustive and these techniques can be successfully applied in many other clinical fields. There are, in contrast, applications of SP to the valuation of more integral benefits, which are not related to a specific clinical field, i.e. health state or health problem. Those are assessments of WTP per QALY (e.g. Gyrd-Hansen \& Kjaer, 2012; Bobinac et al., 2012a, 2012b), prevention or care programs to specific groups (e.g. Corso et al., 2013; Loh \& Shapiro, 2013) or for insurance packages (e.g. Shafie \& Hassali, 2013).

The range of valued benefits in these studies is also broad: models of disease management or treatment (e.g. Mattsson et al., 2013; Moia et al., 2013; Naik-Panvelkar et al., 2012; Whitty et al., 2013), specific diagnostic, treatment procedure or a drug (e.g. Lynn et al., 2013; Hansen et al., 2013; Regier et al., 2012; Marti, 2012; Jendle et al., 2012; Hodgkins et al., 2012; Havet et al., 2012; Hancock-Howard et al., 2012; Glenngard et al., 2012), screening program (e.g. Carroll et al., 2013; Dixon et al., 2012; Sogaard et al., 2012), vaccination (e.g. Sadique et al., 2013; Asgary, 2012), QALY (e.g. Prosser et al., 2013). Health benefits are not always expressed through service provision. Alternatively, benefits are evaluated through WTP for the mere availability of the services (e.g. Kjaer et al., 2012), or for the elimination or prevention of undesired health outcomes (e.g. Brandt et al., 2012; Ethier et al., 2012; Lang et al., 2012).

The presentation of the benefit under valuation is a very crucial question in the design of a SP study as it basically defines what will be valued by respondents. In CV studies, this is done in the scenario specification, while in DCE, the presentation is distributed between the scenario and attributes of the benefit. In any case, the elements that are presented to the respondent depend not only on the study purposes, but also on the relevance of the information to the respondents. Olsen and Smith (2001) classified the characteristics of the valued benefits into: health outcomes, process-utility, and non-health outcomes. Additionally, the monetary and non-monetary cost of acquiring should be specified in case of indirect valuation, i.e. choicebased techniques. Process utility refers to the quality and access characteristics of the health benefit, e.g. service, and may broadly consist of the indicators of clinical and social quality, and spatial, temporal, and psychological access (Berki and Ashcraft, 1980). In this 


\section{Chapter 1}

dissertation, we address a broad range of quality and access characteristics of physician services.

Health care services consumption is unlikely to bring satisfaction per se. Most probably, health care services are valued for their expected health outcomes, i.e. the reduction in the risk of mortality, morbidity, adverse effects or pain. Therefore, most of the valuations are focused on health outcomes (Ryan, 1999). Many studies explicitly describe direct health and related outcomes, like reduction in the risk of the disease (e.g. Regier et al., 2012; Sadique et al., 2013), decrease in symptoms or adverse effects (e.g. Brandt et al., 2012; Corso et al., 2013; Ethier et al., 2012), changes in functional status (e.g. Gyrd-Hansen \& Kjaer, 2012), days of sickness and absence from the job (e.g. Prosser et al., 2013), symptom free days (e.g. NaikPanvelkar et al., 2012), specific efficacy of the treatment (e.g. Marti, 2012; Jendle et al., 2012; Hodgkins et al., 2012; Glenngard et al., 2012). Other studies specify potential changes in the factors that may have health impacts like detection rates for screening and diagnostics (e.g. Carroll et al., 2013; Dixon et al., 2012; Lynn et al., 2013), or population coverage for vaccination (Gidengil et al., 2012). Side effects are sometimes specified additionally to the main health outcomes (e.g. Regier et al., 2012; Marti, 2012; Gidengil et al., 2012; Glenngard et al., 2012) or without the main outcomes (e.g. Moia et al., 2013).

In cancer studies, there is a practice of eliciting WTP for a hypothetical 'magical pill' that can guarantee a full remission or symptom elimination (e.g. Lang et al., 2012; Ethier et al., 2012). In these studies, however, a current state is also specified in health outcome terms as a meaningful comparator. Another interesting strategy is using the self-reported risk perception as the basis for identifying possible health outcomes of the health care service (e.g. Asgary, 2012). Some studies that compare modes of service delivery assume no differences in expected health outcomes and concentrate only on the preferences for the modes themselves (e.g. Whitty et al., 2013; Havet et al., 2012).

Still, there is some portion of the SP studies that do not specify the potential health outcomes and value services or treatment modes per se (e.g. Kjaer et al., 2012; Loh \& Shapiro, 2013; Mattsson et al., 2013; Hancock-Howard et al., 2012; Shafie \& Hassali, 2013). This approach is also justified in case of integral services that may have many different health outcomes and/or are not related to a specific health problem and/or are meaningful and familiar to the respondents. This may include but is not limited to primary care, long-term care and insurance plans. 
The focus of this dissertation on the patient payments for outpatient physician services, also favors the latter approach. First of all, in real life, health care consumers make choices under conditions of uncertainty about the properties and effects of the services. Moreover, primary and outpatient services have very complex outcomes including risk reduction, but also positive benefits from obtaining correct recommendations, referrals or prescriptions. In addition, these services are rather common for the whole population or at least everyone has his/her own prior expectations about their clinical properties. Thus, it is a convenient strategy in our research to use social expectations about the clinical effects without specifying them, concentrating more on other quality and access characteristics of the services.

\subsubsection{Application of discrete choice experiment design}

The design of the DCE involves several steps including the selection of attributes through which the valued health benefit is expressed, constructing benefit profiles, and matching them to obtain choice tasks. These decisions influence the empirical strategies for modeling choices.

As discussed earlier, the attributes of the health care services might include health and nonhealth outcomes, and process utility (Olsen and Smith, 2001). Inclusion of health and related outcomes was discussed earlier. Attributes that belong to the process utility are various and depend on the features of the health benefit provision, often referred to as quality and access attributes in case of the valuation of a service. In case professionals are involved in the provision of the benefit, the professional level is often expressed through their skills and training, or composition of the provision team (e.g. Kjaer et al., 2012; Hancock-Howard et al., 2012; Lynn et al., 2013).

Another group of factors relates to the mode and convenience of the administration of a benefit valued. In case of valuing services or therapies, this may include the description of who delivers it and how (e.g. Hancock-Howard et al., 2012), the frequency of contacts with specialists (e.g. Whitty et al., 2013; Moia et al., 2013; Naik-Panvelkar et al., 2012), the place of the service delivery (e.g. Kjaer et al., 2012; Pavlova et al., 2009). In case of valuing drug therapies, this attribute relates to the convenience of drug intake, frequency, dosing, and route of its administration, (e.g. Naik-Panvelkar et al., 2012; Jendle et al., 2012; Hodgkins et al., 2012; Glenngard et al., 2012). Additional efforts or inconveniences that the therapy might cause, can also be specified (e.g. Moia et al., 2013; Mattsson et al., 2013) as well as additional 


\section{Chapter 1}

conveniences or services may also be presented (e.g. Naik-Panvelkar et al., 2012; , Whitty et al., 2013; Kjaer et al., 2012).

An important aspect of care is the interaction between the patient and the health professional. In this regard, we find two main aspects that might be embedded in the DCE tasks. One of them is the attitude of medical staff and the way they provide information (e.g. NaikPanvelkar et al., 2012; Lynn et al., 2013; Hancock-Howard et al., 2012). Another aspect is the continuity of care, i.e. the possibility to be seen by the same or familiar specialists during the care course (e.g. Whitty et al., 2013; Hancock-Howard et al., 2012).

A special set of attributes, often used in DCE for valuing health benefits is an indication of the time cost. Time cost directly invested by the benefit users, like travel time, waiting in the facility, time of utilization etc., should be distinguished from the passive time attributes, e.g. waiting for the results or the waiting list (e.g. Carroll et al., 2013; Essers et al., 2010; Nieboer et al., 2010; Clark et al., 2009). In the latter case, health benefit users do not invest this time directly as they may perform their usual tasks while being on the waiting list. It should be noted, however, that indications of time cost are often embedded in the delivery mode and placing descriptions, e.g. a home based program requires no travel time as opposed to a hospital-based program (e.g. Whitty et al., 2013). Sometimes, time commitments required are specified explicitly (e.g. Hancock-Howard et al., 2012), especially if there is an objective to estimate the time that may be traded for other attributes (e.g. Prosser, 2013; Ethier et al., 2012).

In DCE, the number of valued attributes and their levels is limited by their feasibility. Even a low number of attributes and/or their levels result in a very high number of all possible profiles (full factorial design) and, consequently, a high number of choices. In the current literature, commonly between four (e.g. Mattsson et al., 2013; Lynn et al., 2013; Carroll et al., 2013; Regier et al., 2012; Glenngard et al., 2012) and eight (e.g. Naik-Panvelkar et al., 2012) attributes are embedded in the DCE tasks, with a tendency to fewer attributes. The attributes are usually selected on the basis of previous theoretical and empirical evidence on the importance of the attributes from prior qualitative investigations and quantitative tests.

A few solutions are applied to reduce the number of possible choice tasks: fractional factorial designs, random assignment (in particular split sample), or both. Full factorial designs are very rare and they definitely require random assignment of the small number of tasks to the respondents (e.g. Prosser et al., 2013; Kjaer et al., 2012). A more common approach is to use fractional factorial designs, which seeks to find the best trade-off between mathematical 
properties of orthogonality, balance, minimal overlap and efficiency (Huber and Zwerina, 1996). Recently, the emphasis has been shifted from orthogonality to efficiency and a number of search strategies have been developed to search the most efficient designs (Bridges et al., 2011, Olaru et al., 2011). The most prominent of them is D-optimality (Huber and Zwerina, 1996). In health care, however, only some of the empirical studies apply D-optimality (e.g. Whitty et al., 2013; Regier et al., 2012; Hancock-Howard et al., 2012). A lot of studies still rely upon orthogonal designs as we do in this dissertation (e.g. Sadique et al., 2013; Moia et al., 2013; Lynn et al., 2013; Carroll et al., 2013; Marti, 2012; Jendle et al., 2012; Glenngard et al., 2012; Gidengil et al., 2012).

The resulting number of choices in a DCE task, after factorial designs and matching, rarely directly leads to a number of choice tasks manageable by the respondents (e.g. Lynn et al., 2013; Carroll et al., 2013; Regier et al., 2012). Therefore, in case of a very high number of choice tasks, a random assignment of the tasks is applied (e.g. Prosser et al., 2013), i.e. each respondent is assigned a target number of choices randomly derived from the full design. A split sample approach (or 'block design') is a very common option if the resulting design contains a number of choices that is high and can be split into two or more sets with manageable numbers (e.g. Whitty et al., 2013; Sadique, Moia et al., 2013; Naik-Panvelkar et al., 2012; Marti, 2012; Kjaer et al., 2012; Glenngard et al., 2012). In the latter case, respondents are randomly assigned to one of the sets with the reduced number of choices. There is no conclusive evidence on which number of choices may be considered as manageable by respondents (Ryan and Gerard, 2003). Recent empirical applications in health care contain a great range of choice tasks numbers starting from four (e.g. Jendle et al., 2012). The upper limit is commonly 16 choice tasks (e.g. Moia et al., 2013; Lynn et al., 2013; Regier et al., 2012). Rarely, a higher number of choice tasks is used, i.e. up to 32 (e.g. Bijlenga et al., 2011); however, this might not be considered as feasible for a respondent.

The absolute majority of the DCE studies are designed so that a respondent makes a binary choice between two unlabeled profiles of a health benefit (e.g. Whitty et al., 2013; Sadique et al., 2013; Prosseret al., 2013; Moia et al., 2013; Mattsson et al., 2013; Lynn, et al., 2013; Carroll et al., 2013; Naik-Panvelkar et al., 2012; Jendle et al., 2012; Hancock-Howard et al., 2012; Glenngard et al., 2012; Gidengilet al., 2012). In this specification, empirical estimation and interpretation is much easier by the application of standard regression models for a binary response variable. However, in real situations, consumers consider a larger number of options. Therefore, to make it more realistic, some studies design a choice set with more than two 
options (e.g. Kjaer et al., 2012). Moreover, where there is the explicit aim of estimating a preferred option (e.g. home-based vs clinic-based care, place of delivery, team vs single specialist consultation), these options might be labeled (e.g. Whitty et al., 2013; Kjaer et al., 2012). This reduces the number of attributes to be varied. However, the preference of the main interest might be also embedded in the choice design as an attribute.

Forcing a respondent to make a definite choice between the presented options is believed to inflate WTP estimates obtained from DCE (Ryan and Skåtun, 2004). A solution to this is the introduction of the opt-out option, i.e. the choice of none of the presented alternatives or the status-quo (Lancsar and Louviere, 2008; Ryan and Skåtun, 2004). Despite its advantages, the opt-out option is rarely applied (e.g. Regier et al., 2012; Marti, 2012). It also slightly increases the complexity of the analytical modeling, which probably can explain the relative unpopularity of this approach. Another challenge is that the opt-out option cannot always be meaningfully expressed in terms of the attributes, which disables its inclusion in the conjoint analysis.

There has been a development in another technique called the best-worst scaling method, which might be regarded as an extension of the traditional DCE, though in some versions it might be seen as a separate elicitation method (Flynn, 2010; Flynn et al., 2007). Originally, it required the presence of the attribute that has the most and the least importance (Finn and Louviere, 1992). It was developed to the profile valuing case, where a respondent chooses the most and least attractive attribute within a presented profile (e.g. Günther et al., 2010). The closest to the traditional DCE, is the multi-profile case where a respondent needs to choose the most and least attractive profiles from the set based on the profile attribute levels (Louviere et al., 2008). However, best-worst scaling method has not found many applications in the health care field so far, though it is actively promoted in the theoretical literature mentioned above.

Empirical strategies for modeling DCE data require an application of the models for discrete outcome measures, and the exact choice depends on the number of choice options. In a binary response specification, the choice is limited to either logit models (e.g. Sadique et al., 2013; Mattsson et al., 2013; Lynn et al., 2013; Regier et al., 2012; Naik-Panvelkar et al., 2012; Kjaer et al., 2012) or probit ones (e.g. Prosser et al., 2013; Moia et al., 2013). The latter is believed to be better as it does not suffer from the IIA (independence of irrelevant alternatives) assumption (Hanley et al., 2003). However, it is applied more rarely, probably due to the higher computational and interpretational complexity. In case of more than two 
choice alternatives, regression analysis extends to multinomial (ususally logit) specification (e.g. Regier et al., 2012; Kjaer et al., 2012). Alternatively, nested (usually logit) specifications are sometimes considered when the opt-out option is present and the decision process is treated as nested (e.g. Marti, 2012). In any of these cases, a model should account for the hierarchical structure of the data as several responses are obtained from one respondent. This is mostly done by extending the model to a fixed/random effect or random parameter specification.

A relatively new promising approach, which is believed to better capture the heterogeneity of the preferences by splitting observed relations into several classes, is latent class modeling (Louviere, 2006). Its applications in DCE studies are growing in number (e.g. Whitty et al., 2013; Carroll et al., 2013). However, to our knowledge none of these applications in health care have accounted simultaneously for hierarchical data structure. Nonetheless, there are advances in the estimation procedures that allow estimating latent class models with random parameters (e.g. Erichsen and Brockhoff, 2004).

\subsubsection{Application of the contingent valuation design}

In a CV study, one of the major design decisions is the choice between elicitation formats, which can be open-ended, via payment cards, one bound discrete choice questions (referendum question yes-or-no), double bound discrete choice, or bidding game. Each of these formats has its own shortcomings. The open-ended format is believed to suffer from strategic and hypothetical bias. The payment card format is subject to a range bias, while the bidding game - to a starting point bias. The discrete choice format is argued to be the best option which overcomes the mentioned biases, and is also theoretically sounder, as it closely resembles choices in real markets, and avoids erratic answers to impact the overall WTP value. This is largely due to the seminal publication in environmental economics by NOAA panel (Arrow and Solow, 1993). On the other hand, the discrete choice format might suffer from “yea-saying” (Blamey et al., 1999) which implies that respondents respond positively to a bid which is higher than their true WTP.

Smith and Sach (2010) showed that in earlier applications, open-ended and payment card formats were most frequent, and with the development of the method to 2005 the number of other formats has risen substantially gradually superseding the use of open-ended format. By this review, in 2000-2005, the payment card and dichotomous choice were the most applied formats. In recent years, this tendency seems to be remaining. A lot of emphasis is now on the 
bidding games and double-bounded dichotomous choice (e.g. Whitty et al., 2013; Shafie \& Hassali, 2013; Hansen et al., 2013; Havet et al., 2012; Ethier et al., 2012; Corso et al., 2013; Loh \& Shapiro, 2013; Lang et al., 2012; Wang \& Hong, 2012). Simple dichotomous choice is applied rarer than the double-bound one (e.g. Asgary, 2012; Gyrd-Hansen \& Kjaer, 2012). Still a lot of applications rely upon payment cards (e.g. Bobinac et al., 2012b; Brandt et al., 2012; Dixon et al., 2012; Gyrd-Hansen \& Kjaer, 2012). The purely open-ended elicitation technique is mostly applied now as a complement to other formats in order to either retrieve more precise estimates after the answer has been framed or for comparison reasons (e.g. Bobinac et al., 2012b; Loh \& Shapiro, 2013). In this dissertation, we apply an open-ended format with payment-cards to obtain conservative WTP estimates from the CV. Conservative WTP estimates are preferred because of our focus on policy assessment.

Empirical strategies for modeling WTP obtained from CV data are various due to different elicitation formats. Nonetheless, linear regression with log transformation of WTP for the open-ended, payment card, or bidding format remains one of the most popular approaches (e.g. Bobinac et al., 2012b; Brandt et al., 2012; Dixon et al., 2012; Hansen et al., 2013; Shafie \& Hassali, 2013). It requires that an exact WTP measure is retrieved from the CV task. If WTP is treated as an ordered outcome, another popular approach is to use an interval regression (e.g. Corso et al., 2013; Ethier et al., 2012; Lang et al., 2012). For the choice format CV, i.e. dichotomous, double bound, a logistic specification is applied (e.g. Asgary, 2012; Gyrd-Hansen \& Kjaer, 2012; Loh \& Shapiro, 2013).

The necessity to deal adequately with zero answers (Strazzera et al., 2003) arises from the fact that often there are spikes at zero and the decision whether to pay and how much to pay may be driven by different factors. The simple approach which may be combined with the empirical strategies described above is excluding zeros from the analysis (e.g. Brandt et al., 2012; Shafie \& Hassali, 2013). Alternatively, the independence of the decisions about paying and choosing the level of WTP is presumed by modeling them separately (Donaldson et al., 1998). Another approach is the application of the family of tobit regressions, i.e. regressions for the censored outcomes. Type I tobit models do not account for the predictors of the decision whether to pay, but model the level of positive WTP conditional on non-zero answering. Type II tobit models, including heckman type models, in contrast, seek to find the predictors of both decision stages (e.g. Havet et al., 2012). Double hurdle models have a similar logic, but also allow for distinguishing between true and protest answers in the first stage decision (Dalmau-Matarrodona, 2001). 
Survival analysis is applied relatively rarely to CV data in health care (e.g. Wang \& Hong, 2012; Mataria et al., 2007), though it has been extensively used in environmental economics (Carson et al, 2003; Carson et al., 1994; Hanemann et al., 1991). In this approach, 'survival' in relation to the WTP level instead of time is estimated, thus, enabling calculations of the proportions of people who are willing to pay certain amounts with respect to their characteristics. This approach basically provides information on the demand shape and shifters if stated WTP is assumed to represent real WTP. In this dissertation, survival analysis of the WTP estimates is presented.

\subsubsection{Other aspects of SP studies}

Recently there has been some work done in the direction of joint data analysis, i.e. SP and RP (Adamowicz et al., 1994), and CV together with DCE (e.g. Mogas et al., 2009; Adamowicz et al., 1998). As the focus of this dissertation is on SP, we touch the question of the latter combination. The main motivation of combining the two is not to lose information specific to each of the methods. As Mogas et al. (2009) states, CV cannot provide information on each and every possible combination of the attributes of the benefit, but only for a certain limited number. Therefore, they offered using CV values as an indication of basic WTP and adjust for changes of the attributes by using results of DCE. This approach to our knowledge has not been applied in health care. Another way is to make a joint estimation on combined DCE and CV data applying a scale parameter representing difference in elicitation methods (Adamowicz et al., 1998). This requires that data in both elicitation methods have the same discrete choice format.

Most of the applications of SP in health care focus on a benefit in a certain country, or even settlement area. Some studies compare cross-country contexts. In many cases, the comparison is made for similar countries in the similar level of development (e.g. Hodgkins et al., 2012) or from the same macro-region (e.g. Glenngard et al., 2012). Few studies, however, have attempted to compare countries which are substantially different in terms of culture and economy (e.g. Corso et al., 2013). This evidence suggests that values are not transferrable across different cultural and economic contexts, though might be rather similar for the countries with similar contexts (Glenngard et al., 2012). This study focuses on the CEE countries, which had similar socio-economic and political context in the $80^{\text {th }}$ of the previous century and has had different development trajectories ever since. Therefore, CEE countries 


\section{Chapter 1}

represent a suitable basis for cross-country comparisons of WTP for health care services. Country context is presented in the Section 1.3.

Concluding, the application of SP in health care has taken various patterns and is growing in number in recent years. Researchers have to make decisions on a number of aspects in the study design, i.e. elicitation technique, elicitation format including choice or non-choice based, the perspective of the study and whose values to be assessed, scope of the study etc. Within each technique, specific choices have to be made with regards to the benefit representation and further data analysis. Despite growth in use, the majority of the applications still are very cautious about the interpretation of the obtained WTP values. They are very rarely addressed in the framework of demand modeling, i.e. pricing decisions or consumption effects. Most probably, this is due to the lack of trust in WTP obtained from the hypothetical tasks. In this dissertation, we attempt an application of stated WTP to the task of the assessment of patient charges for services in the basic package.

\subsection{Patient payments in Europe and country context}

\subsubsection{Patient payments in Europe and CEE}

The official cost-sharing arrangements and informal flow circulation in a country depend on the features of the health care system, but also on the socio-economic and political context (Tambor et al., 2011; Stepurko et al., 2013). European countries differ substantially in their reliance upon public and private funds in the system. In general, more prosperous economies that also spend more on health care per capita afford a higher proportion of health expenditures to be done through public funds (OECD, 2011). In less developed countries, patients bear a higher burden by paying out-of-pocket both formally and informally. However, this balance also highly depends on the institutional and political context.

According to the WHO National Health Accounts data (WHO, 2013), Western European countries are rather homogenous in the mix of private and public funds. The highest reliance on public funds, i.e. more than $80 \%$ of the total health expenditure is financed through public funds, is observed in most of the Nordic countries, Luxembourg, the Netherlands, the UK, and Monaco. France, Germany, Belgium, and Finland where social health insurance is in effect, and Austria, Italy and Spain with universal tax-based systems, cover around $75 \%$ to $80 \%$ 
through public funds. A higher financial burden is shifted to patients in Ireland, Switzerland and Portugal.

CEE countries are very diverse in the structure of their health care finance according to the WHO statistics (WHO, 2013). Most of these countries have had a common socialistic past with universal health care systems offering free services at the point of purchase. In the late $80^{\text {th }}$, all those systems, which were rather inefficient and isolated, faced the necessity of profound organizational and financial reforms (Rechel \& McKee, 2009). However, due to differences in political, social and cultural backgrounds, and economic capacity, the direction and depth of the reforms varied considerably. Such discordant transitions have led to a variety of health systems in CEE with different financing structure. Therefore, the reliance on public funds observed in CEE in 2011 varies from only about 45\% in Albania and Moldova to more than 80\% in Romania (80.2\%), Czech Republic (83.5\%), and Croatia (84.7\%) (WHO, 2013).

The economic situation in most CEE countries was rather hard at the beginning of the transition which provoked a reduction in the public financing, the unique source of health financing at that time (Rechel \& McKee, 2009). This had to be substituted by private sources, primarily by out-of-pocket expenditure. In the lack of a legislative background for official fees and public resistance to market-oriented changes, informal markets immediately spread in most of these health systems (Lewis, 2007). Attempts to formalize or implement official charges were met with a severe resistance in many of the states among public and medical staff (Lewis, 2002).

Nonetheless, due to the lack of public resources in CEE countries, patient cost-sharing for pharmaceuticals has been established in most CEE countries (Espin and Rovira, 2007). In former Soviet countries, Russia and Ukraine, pharmaceuticals in most cases are purchased out-of-pocket (Popovich et al., 2011; Lekhan et al., 2010a). However, patient charges for health care services in the basic health care package are less common, and they are not observed in countries such as Hungary, Poland, Romania, Slovakia, Serbia, and the former Soviet republics, i.e. Estonia, Lithuania, Moldova, Belarus, Ukraine, and Russia (Tambor et al., 2011).

Implementation of official charges for public health care services in CEE, but also in some countries of the Western Europe, is very controversial and usually unpopular in policymaking (Tambor et al., 2011). Few CEE countries, where patient co-payments for basic health care services were not abolished after the recent implementation are Croatia (in 2008) (Vončina et al., 2012), Czech Republic (in 2008) (Bryndová et al., 2009), Albania (in 2008) 
(Tomini et al., 2012), and Estonia (in 2010) (Thomson et al., 2010). For longer periods they existed in Bulgaria (since 2000) (Atanasova et al, 2011), Macedonia (since 1992) (Gjorgjev et al., 2008), and Slovenia (since 1993) (Albreht et al., 2009). In Hungary and Slovak Republic, co-payments were introduced in the late 2006, but after a year of existence, they were abolished due to hot public debates without consideration of their economic effects (Baji et al., 2011). In Romania, the legislative base is ready to host cost-sharing since 2006 (Vladescu et al., 2008) but the government did not press the issue until 2013 when flat co-payments were introduced for one year (Lever, 2013).

Official patient charges are a controversial issue in Western Europe as well. They are not implemented in countries such as Denmark, Malta, Spain, UK, and Greece (Tambor et al., 2011). Similarly to Hungary and Slovak Republic, co-payments for hospital services in Portugal existed since 2007 and were abolished in 2009 due to the lack of economic effect (Barros et al., 2011). Germany has implemented flat co-payments for all types of care starting in 2004, but in 2013 patient charges for physician services were discontinued due to a surplus in the statutory insurance funds. There has been a lot of research producing conflicting evidence about the presence or absence of the effect of patient charges on consumption and revenues in Germany (e.g. Augurzky et al., 2006, Rückert et al., 2008), which only proves that this is highly controversial and political issue.

Official patient charges in CEE take different forms (Tambor et al., 2011). Co-insurance schemes are implemented in Croatia (Vončina et al., 2012), Macedonia (Gjorgjev et al., 2008), and Slovenia (Albreht et al., 2009). In Latvia, co-insurance is implemented in combination with flat rates per visit or admission (Mitenbergs et al., 2012). Flat rate copayments are applied in Bulgaria (Atanasova et al., 2011), and Czech Republic (Bryndová et al., 2009), and were also tried out Hungary and Slovak Republic (Baji et al., 2011). Most of the CEE countries also have a 'ceiling' for annual expenditures to protect patients from overspending. Most often, formal patient charges are levied for both the out- and inpatient services (Tambor et al., 2011), like in Bulgaria (Atanasova et al., 2011), Croatia (Vončina et al., 2012), Macedonia (Gjorgjev et al., 2008), Czech Republic (Bryndová et al., 2009), and Slovenia (Albreht et al., 2009). Less often, only outpatient services are subject to cost-sharing, like e.g. in Albania (Tomini et al., 2012b). No case where only inpatient care is charged to the patients is known in CEE.

Despite the official arrangements in CEE countries or, probably, due to their opacity, informal payments continue to represent a substantial part of health care expenditure with the exception 
of the Czech Republic (Tambor et al., 2011; Lewis, 2007; Lewis, 2002). These payments are especially prevalent in the poorest post-Soviet countries (Rechel \& McKee, 2009), though they are common for most CEE countries. In many of these countries, formal and informal charges coexist (Tambor et al., 2011) causing a double burden for patients. In the former Soviet Republics, a new form - quasi-formal payments has developed (Pavlova et al., 2011; Balbanova et al., 2004; Litvak et al., 2001). It implies that the facilities officially charge patients for the services (usually for better quality services), although government regulations are either lacking or forbid this. Informal and quasi-formal charges represent a substantial threat to the sustainability of health systems as they are not accounted for. Moreover, they are shown to be rather regressive as they impair access to lower-income groups of the population (Atanasova et al., 2012; Baji et al., 2012b; Szende and Culyer, 2006; Murauskiene et al., 2012; Danyliv et al., 2012b; Lewis, 2000).

In this dissertation, we focus on assessing the effects of the patient payments for physician services in CEE countries. From a policy perspective the interest is on the potential effects of official payments. However, given the extent of informal payments in CEE, it is also important to understand the preferences between paying formally and informally. The six countries that enter the study are Bulgaria, Hungary, Lithuania, Poland, Romania, and Ukraine. At the time of data collection, official patient charges for physician services existed only in Bulgaria, were already abolished in Hungary, were about to be implemented in Romania, and were never implemented in the other countries. However, the SP framework applied in this dissertation, allows specification of the hypothetical scenarios of formal charges and makes a cross-country comparison possible. Below, we present the country context in terms of the economic development, general organization of health care financing, and patient payment patterns in these countries.

\subsubsection{Health care funding in the six CEE countries}

The six CEE countries included in the study for this dissertation, well represent the heterogeneity in the region as they greatly differ in the level of economic development and health expenditure (see Figure 1.1panel a), and social and political background. According to the World Bank (2013) data, the lowest income per capita and, consequently, lowest expenditure on health care is observed in Ukraine in 2010. Lithuania has a similar post-Soviet historical background but is a much more developed country with a substantial per capita income and health expenditure approaching Poland. Romania and Bulgaria represent post- 


\section{Chapter 1}

socialistic countries with a moderate development level and are very close in the level of per capita income and per capita total health expenditure. Poland and Hungary are the relatively highly developed CEE countries with the highest income and health expenditure among the six countries in the study (the World Bank, 2013).

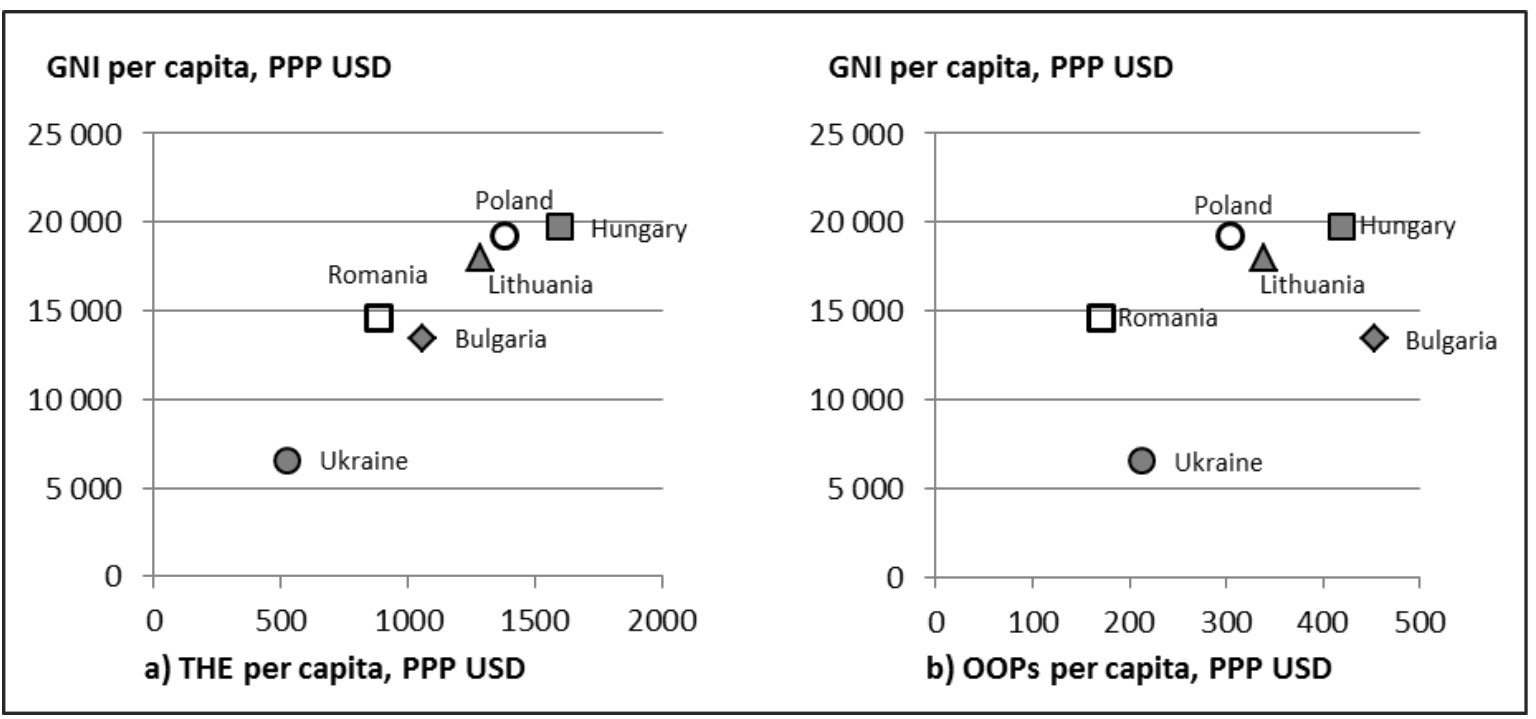

Figure 1.1 Gross national income per capita and total health expenditure per capita in the six countries in 2010, PPP USD (The World Bank, 2013)

However, the burden laid on patients in terms of health expenditure does not fully correspond to the level of the country development. If we consider out-of-pocket expenditures only (see Figure 1.1 panel b) based on the World Bank (2013) statistics, Bulgaria has the highest expenditure per capita of about 453 USD in 2010. Ukraine, where people on average spend 213 USD per year out-of-pocket, also shifts substantially upwards taking over Romania where the level of income is much higher but people spend only about 169 USD out-of-pocket per year. Poland, Lithuania, and Hungary as the most developed countries in the study, have substantial out-of-pocket expenditure per capita (304 USD, 339 USD, and 419 USD respectively).

Table 1.1 shows the structure of financial sources for the total health expenditure in the six countries in this study. All of these countries, except Ukraine, implemented social health insurance. However, all of these systems do not rely solely on the social security funds and can be called mixed systems. According to WHO data (WHO,2012), Romania provides most of the health care through public funds with $64 \%$ being covered through the social health 
insurance and the smallest share of private and out-of-pocket expenditure. A high reliance on social insurance funds is also observed in Poland (64\%), Lithuania (61\%), and also in Hungary (54\%). In these countries, patients cover moderate parts of health expenditure from their own pocket (22\%, 26.4\%, and $26.16 \%$ respectively). In Hungary, also much emphasis is laid on private health insurance, which already covers $2.5 \%$ of total health expenditure. In Bulgaria, only $38 \%$ of total health expenditure is covered by social health insurance. This is the country with the highest reliance on out-of-pocket expenditure (almost 43\%). In all these five countries, the government covers a small part of health expenditure: from $10 \%$ in Poland to $17.5 \%$ in Bulgaria. Ukraine is different in a way that here the Soviet universal system is preserved. Hence, the government spends around 56\% of the total health expenditure from the funds collected through general taxation. However, a substantial share is covered by the patients out-of-pocket (around 40.5\%).

Table 1.1 Structure of the financial sources for total health expenditure, $\%$ of total health expenditure (WHO, 2012)

\begin{tabular}{lrrrrrrrr}
\hline & \multicolumn{3}{c}{ Public expenditure } & \multicolumn{4}{c}{ Private expenditure } & $\begin{array}{c}\text { Other } \\
\text { expenditure }\end{array}$ \\
\cline { 2 - 8 } Country & $\begin{array}{c}\text { General } \\
\text { government }\end{array}$ & $\begin{array}{c}\text { Social } \\
\text { Security } \\
\text { Fund }\end{array}$ & Total & $\begin{array}{c}\text { Out-of- } \\
\text { Pocket }\end{array}$ & $\begin{array}{c}\text { Private } \\
\text { insurance }\end{array}$ & $\begin{array}{c}\text { Other } \\
\text { private }\end{array}$ & Total & \\
\hline Bulgaria & 17.57 & 38.11 & $\mathbf{5 5 . 6 9}$ & 42.88 & 0.46 & 0.97 & $\mathbf{4 4 . 3 1}$ & $\mathbf{0 . 0 0}$ \\
Hungary & 10.69 & 54.08 & $\mathbf{6 4 . 7 7}$ & 26.16 & 2.47 & 6.60 & $\mathbf{3 5 . 2 3}$ & $\mathbf{0 . 0 0}$ \\
Lithuania & 11.97 & 60.93 & $\mathbf{7 2 . 9 0}$ & 26.40 & 0.65 & 0.06 & $\mathbf{2 7 . 1 0}$ & $\mathbf{0 . 0 0}$ \\
Poland & 9.89 & 61.79 & $\mathbf{7 1 . 6 8}$ & 22.07 & 0.67 & 5.05 & $\mathbf{2 7 . 7 9}$ & $\mathbf{0 . 5 3}$ \\
Romania & 16.15 & 64.20 & $\mathbf{8 0 . 3 5}$ & 19.21 & 0.00 & 0.36 & $\mathbf{1 9 . 5 7}$ & $\mathbf{0 . 0 8}$ \\
Ukraine & 56.63 & 0.00 & $\mathbf{5 6 . 6 3}$ & 40.50 & 0.88 & 1.99 & $\mathbf{4 3 . 3 7}$ & $\mathbf{0 . 0 0}$ \\
\hline
\end{tabular}

\subsubsection{Patient payment arrangements in the six CEE countries}

The Bulgarian health care system (see Georgieva et al., 2007, Pavlova et al, 2000, Atanasova et al, 2011) started a transition from the Soviet model of centralized health care provision in 1990. Health care facilities were transferred to local and municipal authorities and many of them were privatized. Nowadays, GPs are paid through a capitation system, outpatient specialists through a fee-for-service system, and hospitals are financed according to clinical pathways with the target to establish a DRG system (Atanasova et al, 2011). A National Health Insurance Fund with 28 decentralized regional departments was established in 2000. The funds are collected via payroll contributions which are $8 \%$ of the monthly wage divided in a 60:40 ratio between employer and employee. Health care for low-income and socially 


\section{Chapter 1}

disadvantaged people is covered by the state and local budgets. Social health insurance covers a basic package, which is periodically reduced due to the lack of sufficient resources (Atanasova et al, 2011). There is concern about the high share of uninsured people consisting mostly of low-income individuals and the Roma population (Rechel \& McKee, 2009). The services outside the package require either additional insurance or direct payments. The use of services without a referral requires a full compensation from the patients. In 2000, with the purpose to contain resources and due to the introduction of social health insurance, several measures were introduced, among which were quotas for specialists' referrals and the introduction of the co-payments. Patients pay $1 \%$ of the minimum monthly salary (around $€ 1.2$ at present) for an outpatient visit and 2\% (around $€ 2.4$ ) for a day of hospitalization. However, a broad list is in effect exempting many categories of Bulgarians, i.e. unemployed, children, people with special illnesses, disabled, veterans, imprisoned, low-income people, and medical personnel from paying these charges. Informal payments are also widely spread in Bulgaria amounting to about 3.6\% of public health expenditure (Atanasova et al, 2011; van Mosseveld et al., 2008) which is about 2\% of total health care expenditure. Patients pay informally to reduce waiting time, for direct referral to specialists, and for services of better quality.

Hungary started a transition from the tax-financed system to social health insurance (Bismarck model) in 1989. Still, most of the health care facilities are owned by municipalities or the state (Baji et al., 2012b). Family doctor services are paid through capitation, outpatient specialist care is paid through a fee-for-service point system, acute inpatient services is paid through a payment system based on diagnosis-related groups (DRGs) and chronic care through per diem rates (Gaál et al., 2011). The National Health Insurance Fund covers the recurrent cost of services, while capital expenditure through local and central government funds (Baji et al., 2012b). The coverage of the population should be comprehensive; however, the insurance status of around $4 \%$ of the population is not clear. Instead of a basic package, positive and negative lists are used, with the former being non-exhaustive, but rather broad. A considerable share of out-of-pocket expenditure is attributed to co-payments and co-insurance for pharmaceuticals, but also to widely spread informal payments (Gaál et al., 2011). Patients are obliged to co-pay for pharmaceuticals, medical aids, prostheses, balneotherapy and medical recreation, dental care, long-term chronic care, hospital services; but also costsharing is required in case of a visit to a specialist without referral or if patients choose other providers than they were referred to or other services than they were prescribed. In 2006- 
2007, flat co-payments for outpatient visits and a per diem rate for inpatient care were introduced which allowed collecting an additional 0.7\% of total health expenditure in 2007 (Gaál et al., 2011). But due to fierce political debates, these co-payments were abolished at a national referendum (Baji et al., 2011). Informal payments in Hungary are tolerated by the general public and authorities and according to different estimates they may constitute from 1.5 to $4.6 \%$ of total health expenditure (€ 64.8M - € 203.6M in 2001) (Gaál et al., 2006).

Lithuania has started a transition of the health care system in 1996 and a Statutory Health Insurance Fund was introduced a year later (see Murauskiene et al., 2013; Murauskiene et al., 2012). The Health Insurance Fund at the beginning was co-financed from payroll contributions and transfers from the government with the latter being the major source of funding. However, gradually the Health Insurance Fund gained stability, though its deficit is still financed from the state budget. Co-payments are charged for pharmaceuticals, sanatoria stay, and dental services. For outpatient and inpatient services, the option of co-payments was discussed but never introduced. The national legislation declares free of charge health care in the public facilities to all citizens, though a small negative list of services which are fully charged is established (Murauskiene et al., 2010). On the other hand, the regulations are very controversial and public facilities often charge patients for the services outside the negative list and there is debate whether these charges are legal or illegal. These user charges may be considered as quasi-formal and represent a substantial share of health expenditure in Lithuania (Murauskiene et al., 2012). Informal payments also exist in Lithuania. However, estimates of their scope are lacking.

The Polish health system (see Sagan et al., 2011) also started an early transition in 1989 from the centralized system funded through general taxation to a system with prevailing social health insurance. The two Health insurance funds, general and agricultural, pool the funds and transfer them to the central National Health Fund, who is the principal payer in the system. Primary care providers are paid based on a capitation principle and inpatient care is paid based on DRGs. The system stimulates competition between providers at the regional level for contracts with local branches of the National Insurance Fund. Social health insurance covers about $98 \%$ of the population and the benefit package (positive list) is very comprehensive, at least on paper (Golinowska and Tambor, 2012). The contributions are collected via a payroll tax paid by the employee entirely. Cost-sharing is only applied to medicines, medical products, auxiliary medical devices, heath resort treatments, and certain dental services. Informal payments were widespread in the Polish health system at the 
beginning of the transition (Lewis, 2002) but were substantially reduced by the anticorruption measures implemented in 2005 and are now less spread in comparison to other CEE countries (Stepurko et al., 2011; Golinowska, 2010). Informal payments are primarily done in public hospitals, though are present in outpatient services as well. In 2009, they were estimated to account for around $1.5 \%$ of the out-of-pocket payments or $0.33 \%$ of the total health expenditure (Sagan et al., 2011; Golinowska, 2010).

The transition in Romania started in 1989 and in 1998 social health insurance was introduced (see Vladescu et al., 2008). The National Insurance Fund has 42 District Funds and the process of collection and contracting local providers is decentralized. Employees pay 6.5\% in payroll tax, and the employer pays another $7 \%$. The basic benefit package includes health care services, medicines, and medical devices. Patients have a free provider choice. Patients are entitled to co-payments for some pharmaceuticals (10\% and 50\% groups) and medical goods. Reference pricing is implemented and, thus, patients cover the cost of drugs over the reference price. In 2004, co-payments for pharmaceuticals were reduced and the list of fully reimbursed drugs was substantially extended. As mentioned earlier, there were also debates about the introduction of co-payments for health care services and inpatient admissions. The latter were recently implemented (Lever, 2013). Informal payments in Romania are a cultural issue. According to the World Bank (Belli, 2003), they account to over $40 \%$ of out-of-pocket payments, or about $7-8 \%$ of the total health expenditure.

The Ukrainian health care system has never gone through a transition and remains practically unchanged from the Soviet times (see Gryga et al., 2010; Lekhan et al., 2010a). Thus, it proclaims free-of-charge medical care for every citizen in public and communal facilities. Most of the facilities stay publicly or communally owned and are financed from the state and local budgets on a strict line-item budget principle. This is an input-based financing system, highly centralized and bureaucratic, with no sensitiveness to the real needs of the population. Private sector as well as private insurance are underdeveloped and cover less than $2 \%$ of the population mainly from high-income groups. Officially, public sector facilities may not charge patients except for a limited list of auxiliary and paramedical services. However, "charitable donations" are often required. The practice of informal payments to medical staff (under-the-table payments, bribes, gratitude) is widespread. Practically all pharmaceuticals and consumables are purchased out-of-pocket by patients. Informal payments are especially high in inpatient care. By WHO estimates, informal payments accounted for 8 to $10 \%$ of total health expenditure in 2003-2005 (Gotsadze et al., 2006). However, the true number might be 
higher as facilities often require formal charges for better services, which is illegal in principle, but tolerated by the authorities.

Thus, official patient payments have had different levels of success in European countries. In CEE countries they are a relatively new and uncommon phenomenon. In the six countries that are included in this study, the experience with official charges for outpatient health care services has been limited. That is the official charges existed very shortly in Hungary and Romania (since 2013), level of charges did not vary substantially in Bulgaria, and they were never implemented in Lithuania, Poland, and Ukraine. Instead, facilities find their way to charge quasi-formal payments which are especially substantial in Lithuania, Romania and Ukraine. Informal payments are most widespread in Ukraine and Romania, to a lesser extent in Lithuania and Hungary, and in Bulgaria and Poland least of all (Stepurko et al., 2013). Thus, patients in these countries are familiar with patient charges for physician services, though evidence on the effects of such charges is absent due to mostly limited experience with them.

\subsection{Aims and objectives of the dissertation}

The research presented in this dissertation is motivated by the lack of evidence on the potential consumption effects of patient payments for physician services in CEE countries (as outlined above). Given the absence of data on actual behavior under different payment regimes in these countries, this dissertation adopts the SP approach. The application of SP to payment policy assessment is rarely reported in the literature and this dissertation tackles the methodology concerns regarding this application. Thus, the dissertation has both methodology and application aspects.

\subsubsection{Aim}

The overall aim of this dissertation is to apply stated preferences techniques to study the willingness to pay for physician services in six Central and Eastern European countries (Bulgaria, Hungary, Lithuania, Poland, Romania, and Ukraine), and to analyze to what extent WTP estimates can be used in the assessment of patient charges for physician services. 


\subsubsection{Objectives}

To address the main aim of this dissertation, the following objectives are defined:

1. To investigate aspects of the value added of physician services by determining the marginal willingness to pay for service quality and access characteristics using a discrete choice experiment technique.

2. To study the socio-economic and demographic drivers of the willingness to pay and level of the willingness to pay for physician services using a contingent valuation technique.

3. To compare the willingness to pay estimates from the two main stated preference techniques, i.e. discrete choice experiment and contingent valuation, in terms of their consistency.

4. To use the stated willingness to pay values to define the potential demand pools at different levels of patient charges for physician services.

5. To assess the possible overestimation of the willingness to pay by respondents using calibration techniques.

6. To compare willingness to pay estimates with service cost and actual payments.

7. To discuss the implications of the willingness to pay estimates for determining feasible levels of patient charges for physician services and for the overall assessment of patient payment policies.

\subsubsection{General methodology}

The analyses in this dissertation are based on two datasets with a similar structure: a smallscale but representative survey from Ukraine, and a large-scale representative survey from six CEE countries, namely Bulgaria, Hungary, Lithuania, Poland, Romania, and Ukraine. The objectives 1 through 3 are dealt with in chapters 2 through 4 respectively, and the analyses are based on the data from the small scale survey in Ukraine. The fourth and fifth objectives are the main research questions in chapters 5 and 6 of this dissertation respectively. To tackle these objectives at a cross-country level, we use the WTP estimates from the CV tasks in a large-scale survey held in the six CEE countries.

The first dataset, the small-scale survey in Ukraine, was held in December 2009, and contains responses from 303 respondents, representative of the adult Ukrainian population. The questionnaire contains information on SP for outpatient physician services, but also about the past utilization of these services, characteristics of the respondents and their households. A 
multi-stage area probability sampling strategy is used with 110 settlements as primary units and 270 voting precincts as secondary units. For logistic reasons, the data collection was performed within a larger household survey. A waiver from a full ethical review was obtained prior to the survey. A more detailed report on the sampling and data collection issues may be found in the Appendix A.

The second dataset contains information from the survey from six CEE countries, around 1000 respondents representing the adult population in each country, 6052 respondents in total. It was held in July-August 2010. This survey contains questions similar to the small-scale survey, as well as additional questions, which are not analyzed in this dissertation. No ethical approval is needed in the six studied countries for such type of study. A more detailed report on the sampling and data collection issues may be found in the Appendix B.

\subsection{Outline of the dissertation}

The dissertation is divided into seven chapters (including this first introductory chapter), gradually addressing the objectives set in this chapter. The outline and summary of each chapter is presented below.

Chapter 2 provides evidence on the preferences of Ukrainian consumers for health care improvements, which can help to design reforms that reflect societal priorities. In particular, chapter 2 aims to elicit and to place monetary values on public preferences for outpatient physician services in Ukraine. The DCE data from a small-scale survey from Ukraine are analyzed using a random effect logit model with interactions. Respondents passed 16 choice tasks between two physician profiles represented through seven quality and access characteristics (specialization of a physician, state of the medical equipment, maintenance of the office, attitude of the medical staff, travel time, waiting time, and formal vs informal type of payment), and a cost attribute. The marginal WTP for quality and access improvements is assessed.

In Chapter 3 we analyze the potential and feasibility of official patient charges for public health care services in Ukraine by studying the patterns of fee acceptability, ability and WTP for public health care across population groups. We use CV data collected in the small-scale survey in Ukraine. The respondents were asked to value four profiles of a physician by stating their maximum WTP for a visit to such physician. The profiles varied so that two separate 


\section{Chapter 1}

effects could be estimated: specialization of a physician, and the joint effect of improvement in the state of the medical equipment, maintenance, and waiting time. First the payment cards were presented, followed by an open-ended question about the exact amount. If a respondent reported being unwilling to pay a reason was elicited. Three decision points were separated: objection to pay, inability to pay, and level of positive non-zero WTP. These decisions were studied for relations with quality profiles of the services, and socio-demographic characteristics of the respondents and their households.

As explained above as well as in Chapter 4, DCE and CV are often applied to value health care benefits. However, whether the two techniques yield converging WTP estimates is not studied well. Thus, chapter 4 aims to compare at a disaggregated level the WTP estimates for physician services obtained from a DCE and from a CV study applied in the small-scale survey in Ukraine (the two studies presented in chapter 2 and 3). We study the consistency between the estimates and whether there are systematic differences between the two types of estimates.

As outlined above and in Chapter 5, patient charges for health care services are implemented in high-income countries to reduce unnecessary service use. However, evidence on their potential effects in Central and Eastern Europe is lacking. Thus, in chapter 5, we provide evidence on the potential impact of patient charges on the consumption of specialized physician services in the six CEE countries included in this dissertation: Bulgaria, Hungary, Lithuania, Poland, Romania, and Ukraine. We apply a semi-parametric survival analysis to the stated WATP in order to identify potential demand pools, i.e. shares of population willing and able to pay a certain fee in case they need a service, and we also calculate price, income and age semi-elasticities. Data are collected through national surveys held in 2010 among representative samples of about 1000 respondents in each country.

Chapter 6 is devoted to the assessment of proper levels of WATP for specialized physician services which accounts for possible hypothetical bias. The analysis is based on the same data as in Chapter 5, i.e. CV data from six CEE countries: Bulgaria, Hungary, Lithuania, Poland, Romania, and Ukraine. The WATP level is modeled with a stochastic frontier regression. Two possible bias directions are tested: over- (positive inefficiency term) and understatement (negative inefficiency term). The WATP level is then calibrated based on individual predictions of the inefficiency scores. The raw and calibrated WATP levels are compared to actual expenditure by annual predictions for expenditure if respondents were actually paying amounts equal to their WATP. 
Chapter 7 presents the discussion of the key findings outlined in previous chapters and relates them to the objectives of the dissertation. We discuss the main methodological implications for the application of SP to valuing health benefits, especially in terms of deriving policy recommendations. Policy implications relating to future patient payment policies in Bulgaria, Hungary, Lithuania, Poland, Romania, and Ukraine are also discussed. 



\section{CHAPTER 2}

\section{Preferences for physician services in Ukraine:}

\section{A discrete choice experiment}

Based upon:

Danyliv, A., Pavlova, M., Gryga, I., \& Groot, W. (2014). Preferences for physician services in Ukraine: a discrete choice experiment. The International Journal of Health Planning and Management, published online. DOI: 10.1002/hpm.2239 


\section{Abstract}

Background: Structural, organizational, and financial reforms of the health care system are major questions on the policy agenda of the Ukrainian government today. Therefore, it is important to know the social preferences regarding health care provision, in order to design reforms that reflect societal priorities.

Objective: To elicit and to place a monetary value on the public preferences for outpatient physician services in Ukraine.

Methods: a DCE technique is applied to a sample of 303 respondents, planned as being representative of the Ukrainian population but containing a slight over-presentation of certain age-gender groups. Each respondent answered 16 binary choice questions. Out of four empirical models estimated the one with best statistical fit, the mixed effect logit with interaction terms, was chosen for interpretation. The importance of the quality and access attributes was assessed by calculating the marginal WTP.

Results: At the sample level, no preference is found for paying formally or informally, neither for visiting GP or medical specialist. However, people with a higher education demonstrate a preference for visiting a medical specialist, while disabled people prefer a GP. The preference for paying formally is rather strong for people with a medical background. The most important quality factor is the attitude of medical staff followed by the state of medical equipment, maintenance of the physician's office, travelling and waiting time. Marginal WTP for each of these factors is rather high.

Conclusions: In investment decisions, priority should be given to interpersonal aspects of outpatient care, followed by clinical quality. Social quality and access are important as well, but their improvement brings fewer social gains. Measures should be taken to formalize the informal payment channels and to strengthen the gate-keeping role of primary care. 


\subsection{Introduction}

The reform of the health care system is a major question on the policy agenda of the Ukrainian government. Low service quality, unsustainable budget financing (Vynogradov, 2007; Karamyshev et al., 2006), informal patient payments (Balabanova et al., 2004; Lekhan et al., 2004; Litvak et al., 2001), and an underdeveloped primary care system (Barmina, 2009) are some of the most pressing problems in the Ukrainian health care sector. Nevertheless, since obtaining independence in 1991 (after the collapse of the Soviet Union), all attempts of health care reforms have failed. So far, the efforts to introduce social health insurance and formal patient payment schemes have been rejected in the Parliament due to a lack of political will under the pretext of a violation of the Constitution of Ukraine (Ukraïna, 1996, pt. 2, art. XLIX), which guarantees 'free-of-charge' medical care to every citizen. Thus, an adequate market mechanism in the Ukrainian health care sector is still absent. The introduction of general practitioners in the primary care in 2006 was imposed offhandedly by means of a short training of questionable quality for medical specialists. Still, the number of general practitioners is insufficient.

To cope with the health care problems however, substantial organizational, structural and financial reforms in the health care sector are required. The current comprehensive medical reform was planned to be implemented in 2011-2014 and is piloted in four large regions of Ukraine (Committee on Economic Reforms, 2010; MOH, 2010; Law of Ukraine, 2011). Briefly, it implies a clear division of the levels of care (primary, secondary, tertiary, and emergency), strengthening primary care networks, and a reorganization of health care facilities in accordance with the needs of the population. Health care needs, however, will continue to be defined by the local health authorities. There is also the intention to separate payers and providers by transferring ownership of facilities from the state to the communal level and setting a fixed amount for reimbursement through an annual contract between providers and local health authorities.

Thus, despite the fact that the obsolete centralized mechanisms of health care planning and funding will be retained, there is a clear intention to reorganize health care provision so that it is better able to meet public needs. Moreover, a system of quality indicators is to be introduced in the process of the facility performance assessment which provides an opportunity to introduce clinical indicators together with public preferences and satisfaction. From this perspective, it is important to know the social preferences for improvements in 


\section{Chapter 2}

health care provision, in order to design reforms that respond best to these societal priorities. The task of eliciting public preferences and placing a monetary value on them is especially valuable in a context of limited public budgets (Ryan, 2004c; Telser and Zweifel, 2002). The literature, however, does not offer any evidence on public preferences for health care services in Ukraine.

The aim of this chapter is to contribute to the policy discussions on health care reforms in Ukraine by providing evidence on the preferences of health care consumers. In particular, we aim to elicit public preferences for outpatient physician services (including primary care providers and medical specialists) and to estimate the relative monetary value that consumers place on quality and access improvements of these services. An additional contribution of the study is that we analyze preferences for paying formally or informally and for a first contact with a general practitioner or medical specialist.

We use data from a small-scale household survey among a representative sample of the population carried out in 2009 in Ukraine (see Appendix A for sampling details). In one part of the survey, respondents were presented with hypothetical physicians' profiles (close to 'real-life' profiles) and were asked to make choices among these profiles given a hypothetical scenario about the symptoms they experience. Thus, we apply a SP method known as DCE. Although the method is still being developed, it is firmly rooted in decision theory (Zweifel et al., 2006), which allows for a meaningful interpretation of the results.

\subsection{Research background}

In the absence of an adequate market mechanism, which is the case with physician services in Ukraine and other countries (Louviere and Lancsar, 2009), SP methods are needed to elicit public or patient preferences for a good or service. As discussed in Chapter 1, SP techniques allow modeling hypothetical behavior in other than the existing institutional context (Brau and Bruni, 2008). This is an advantage for the evaluation of the potential for health care reforms. Although most of the previous studies on preferences for physician services are based on RP techniques (Skriabikova et al., 2010), some recent studies use a SP approach, namely self-explicated attribute rating or ranking (e.g. Pavlova et al., 2003), rating or ranking conjoint analysis (e.g. Pavlova et al., 2004), CV methods (e.g. Mataria et al., 2007), or the DCE method (e.g. Cheraghi-Sohi et al., 2008; Hjelmgren and Anell, 2007; Neuman and Neuman, 2008; Vick and Scott, 1998). A review of current applications of SP techniques in 
health care is provided in section 1.2. There we also discuss their advantages and limitations. In this chapter, we apply the DCE method because it is specifically designed to estimate trade-offs between service characteristics (incl. 'non-marketed' or 'non-traded' characteristics) (Hanley et al., 2003; Louviere and Lancsar, 2009; Telser and Zweifel, 2007), which is its distinctive strength when compared to other SP approaches. From a health policy perspective, the analysis of trade-offs is important because it could help to ration health care resources and set priorities for health care service improvements, in this case the improvement of physicians services in Ukraine.

An important contribution of this study is the investigation of the effect of informal patient payments on consumer choice, which has not been addressed so far for any type of care, including physician services (see Skriabikova et al., 2010). Informal payments for health care services are reported in many countries around the world (for a review see Stepurko et al., 2010). As discussed in Chapter 1, informal payments are widely spread in the Ukrainian health care system and represent a substantial share of total health expenditure (Balabanova et al., 2004; Gotsadze, 2006; Lekhan et al., 2004; Litvak et al., 2001). The existence of informal patient payments negatively affects the efficiency and equity of health care provision (Ensor, 2004; Lewis, 2002; Szende and Cuyler, 2006; Thompson and Witter, 2000). Therefore, the mechanisms for dealing with these payments are an important policy challenge. There is a concern that the introduction of formal patient payments will not eradicate informal ones, and could result in a double financial burden on patients (Baschieri and Falkingham, 2006; Ensor and Savelyeva, 1998; Lewis, 2000). Information on whether health care consumers accept informal payments could yield relevant policy solutions. Therefore, our article highlights how Ukrainian consumers value the trade-off between formal and informal patient payments if the former were introduced.

Strengthening the gate-keeping role of primary care is a crucial question in the health care reforms in Ukraine because this role is believed to have positive effects in terms of costcontainment (González, 2010) and efficient resource allocation (Zielinski et al., 2008), though the current literature contains ambiguous evidence on this (e.g. Brekke et al., 2007; Escarce et al., 2001; Ferris et al., 2001; Linden et al., 2003). In the context of outpatient care in Ukraine, there are various types of physicians that act as general practitioners, namely district physicians, district therapeutists, and family physicians. In Ukraine, it is also possible to have direct access to a medical specialist. There is empirical evidence that strict gate-keeping that prevents direct access might lead to lower patient satisfaction, especially when there is 
distrust and little confidence in the knowledge and skills of primary care physicians (Kerr et al., 1999; Grumbach et al., 1999). Direct access to a medical specialist might be especially desirable for people with a chronic illness (Rogers et al., 2004). At the same time, services of general practitioners are often characterized by better continuity and comprehensiveness of care (Starfield, 1992), compared to medical specialists. This is because the general practitioner follows the patient through various health problems and has more complete information on the general health status of the patient. Thus, some groups of patients might prefer consulting a general practitioner first before visiting a specialist (Rogers et al., 2004; Grumbach et al., 1999). Therefore, in this study, we also keep the focus on the public preferences towards the general practitioner or direct referral to a medical specialist at the initial contact.

Although the literature contains a wide range of studies on public preferences for health care services, studies focused on the analysis of preferences especially for physician services using a DCE method, are rare. We identified four DCE studies focused on physician services held in four different settings (Cheraghi-Sohi et al., 2008; Hjelmgren and Anell, 2007; Neuman and Neuman, 2008; Vick and Scott, 1998). In three of these studies, a distinction is made between interpersonal aspects and quality aspects of care (Cheraghi-Sohi et al., 2008; Neuman and Neuman, 2008; Vick and Scott, 1998). However, conclusions about the prioritization differ. Hjelmgren and Anell (2007) added the attributes of consumer's choice and control over the care received, and found these attributes to be the most important for patients. Recent literature reviews (including reviews of studies that do not use the DCE method) offer numerous attributes of physician services that might impact patients' priorities (for a systematic review see Cheraghi-Sohi et al., 2006; Wensing et al., 1998). Again, authors mostly stress the distinction and trade-off between technical and interpersonal aspects of quality of care. Although, there is no conclusive evidence on which aspect is more important to the patients, we use these studies to set up our DCE.

\subsection{Methods}

As explained in Chapter 1, the DCE method is increasingly used in health service research and there is a growing body of literature on the methodology of DCE (for an overview see e.g. Lancsar and Louviere, 2008; Mele, 2008; Ryan, 1999; Ryan and Farrar, 2000). The design of a DCE study follows several basic steps explained in section 1.2, which we specify below. 


\subsubsection{Selection of attributes and attribute levels}

Despite the numerous relevant attributes (described in the previous section), the number of attributes and attribute levels in a DCE study should be kept at a minimum to allow for a manageable number of profiles. Even though there are methods for drawing a meaningful sub-set of profiles for DCE and still obtaining relevant results (see Street et al., 2005), the number of profiles that should be included, increases considerably with every attribute or attribute level added. In our DCE the physician profiles were described through eight quality, access, and price attributes. The attributes and their levels are presented in Table 2.1.

'Physician's specialization' refers to a general practitioner or a medical specialist. We assume that this attribute represents not only the provider's knowledge and skills but also other aspects of clinical performance that are not captured by the other attributes, such as continuity, comprehensiveness and appropriateness of care. This attribute was preferred to other indicators of clinical quality or treatment success for several reasons. In SP studies, real life choices should be mimicked to obtain valid estimates. As outlined in section 1.2, health care consumers practically never deal with such kind of information in real life, while the outcomes of these services are rather complex and can hardly be captured by a single attribute. The pre-test of the DCE questionnaire also confirmed that respondents are confused with the indicators of treatment success. Moreover, the inclusion of 'physician's specialization' attribute into DCE allows exploring if a specific preference for bypassing primary contact with a GP exists in Ukraine which is of high policy relevance (see section 2.2).

The next quality-related attribute 'state of medical equipment' reflects the technology aspects of clinical quality irrespectively of physicians' abilities and skills. We assume that this is a multi-aspect characteristic that indicates jointly the effectiveness and promptness of the treatment, as well as comfort to the patient and physician. Although the probability of success of treatment could be another relevant indicator of clinical quality, we do not include this attribute because: (1) it depends on both physicians' abilities and technological aspects of care, and thus, it does not allow studying their separate effects, (2) our pre-tests showed that such information is not readily available to health care consumers in Eastern European countries, such as Ukraine, and they are not used in decisions and (3) some individuals may have difficulties in understanding its meaning. 
Table 2.1 Attributes of physician services and their levels in DCE

\begin{tabular}{|c|c|c|c|c|c|c|c|c|c|c|c|c|c|c|c|c|c|}
\hline \multirow{2}{*}{$\begin{array}{l}\text { Attributes } \\
\text { Levels of attributes }\end{array}$} & \multirow{2}{*}{$\begin{array}{c}\text { Basic } \\
\text { profile }\end{array}$} & \multicolumn{16}{|c|}{ Alternative profiles from orthogonal array } \\
\hline & & 1 & 2 & 3 & 4 & 5 & 6 & 7 & 8 & 9 & 10 & 11 & 12 & 13 & 14 & 15 & 16 \\
\hline $\begin{array}{l}\text { Physician’s specialization } \\
\qquad \begin{array}{l}1=\text { Medical specialist } \\
0=\text { General practitioner }\end{array}\end{array}$ & $\mathbf{0}$ & 0 & 1 & 0 & 1 & 1 & 0 & 0 & 1 & 1 & 0 & 1 & 0 & 1 & 1 & 0 & 0 \\
\hline $\begin{array}{l}\text { State of the medical equipm } \\
\qquad \begin{array}{l}1=\text { Modern } \\
0=\text { Outdated }\end{array}\end{array}$ & ent & 0 & 1 & 0 & 1 & 0 & 0 & 1 & 1 & 0 & 0 & 1 & 1 & 0 & 0 & 1 & 1 \\
\hline $\begin{array}{l}\text { Maintenance of the office } \\
\qquad \begin{array}{l}1 \text { = Renovated } \\
0=\text { Old-looking }\end{array}\end{array}$ & 0 & 0 & 1 & 1 & 0 & 0 & 0 & 1 & 1 & 1 & 1 & 0 & 0 & 1 & 0 & 0 & 1 \\
\hline $\begin{array}{l}\text { Attitude of medical staff } \\
\qquad \begin{array}{l}1=\text { Polite } \\
0=\text { Arrogant }\end{array}\end{array}$ & 1 & 0 & 1 & 0 & 0 & 1 & 1 & 1 & 0 & 1 & 1 & 1 & 1 & 0 & 0 & 0 & 0 \\
\hline $\begin{array}{l}\text { Travel time to the office } \\
60 \mathrm{~min} \\
15 \mathrm{~min}\end{array}$ & 15 & 15 & 15 & 15 & 60 & 60 & 60 & 15 & 60 & 60 & 60 & 15 & 15 & 15 & 15 & 60 & 60 \\
\hline $\begin{array}{l}\text { Waiting time in front of the } \\
\text { office } \\
45 \mathrm{~min} \\
10 \mathrm{~min}\end{array}$ & 45 & 10 & 10 & 45 & 10 & 10 & 45 & 45 & 45 & 45 & 10 & 45 & 10 & 10 & 45 & 45 & 10 \\
\hline $\begin{array}{l}\text { Size of patient payment }{ }^{\mathrm{a}} \\
100 \mathrm{UAH} \\
60 \mathrm{UAH} \\
20 \mathrm{UAH}\end{array}$ & 20 & 20 & 100 & 20 & 20 & 20 & 60 & 20 & 20 & 20 & 100 & 60 & 20 & 60 & 100 & 100 & 60 \\
\hline $\begin{array}{l}\text { Form of patient payment } \\
\qquad \begin{array}{l}1=\text { Formal } \\
0=\text { Informal }\end{array}\end{array}$ & 0 & 0 & 0 & 1 & 1 & 1 & 0 & 1 & 0 & 0 & 1 & 1 & 0 & 0 & 1 & 0 & 1 \\
\hline
\end{tabular}

a Average daily exchange rate during the period of data collection is $11.5424 \mathrm{UAH}$ / $€$

In addition to clinical quality, we also include the social quality of physician services, which is not directly related to treatment outcomes but might affect consumer choices. This is done by including the attribute 'maintenance of the physician's office', which reflects the state of the facility.

The three access-related aspects, namely psychological, spatial and temporal access, are represented by three distinctive attributes that are independent of each other, namely 'attitude of medical staff', 'traveling time' and 'waiting time' respectively. The attribute 'attitude of medical staff' also indicates the interpersonal aspects of care, as well as the possibility to receive personal attention during the treatment.

In addition to quality and access, we introduce a third dimension relevant to the consumer choice of a physician, namely the price of service. For this purpose, we include 'size of patient payment' as an attribute in our DCE. As discussed at the outset of our article, we also investigate preferences for paying formally or informally and, therefore, we introduce a 
separate attribute 'form of payment' with two levels: formal and informal. The form of payment and the size of payment represent the price dimension in our DCE study.

All attributes, except the size of patient payment, have two levels. The size of patient payment is represented by three levels because during the pre-test these levels were found to be meaningful for respondents. We choose quantitative attribute levels for travelling and waiting time, and for the size of patient payments. The other attributes, are represented by two qualitative attribute levels representing mutually exclusive contrasting end-states.

\subsubsection{Specification of the DCE design}

In order to reduce the number of profiles (i.e. the full factorial of attribute levels is 384) to a manageable number, an orthogonal main-effect fractional factorial design was drawn (Orthoplan, SPSS software), which resulted in 16 scenarios. Orthogonal main-effect fractional factorial designs provide the minimal number of profiles still allowing estimation of the main effects under the assumption that interactions between attributes are absent or negligible. The design was checked for overlap, orthogonality and level balance (for definitions see Huber and Zwerina, 1996).

Due to the fact that the price attribute has 3 levels while all the other attributes have only 2, the level balance was not fully reached. The 16 profiles from the orthogonal design were matched to one fixed profile, different from those 16 profiles that represented a realistic profile for Ukraine. In this way the differences in profiles for each choice task still represent an orthogonal array. Obviously dominant choices were not excluded in order to maintain factor orthogonality. Thus, the respondents were presented with 16 choice tasks each containing the fixed profile and one alternative profile from the orthogonal design.

\subsubsection{The preference elicitation}

An example choice task is presented in Appendix C. Following the framework used by Vick and Scott (1998), the choice tasks are preceded by a scenario, where a respondent is asked to imagine that he/she experiences major or severe health symptoms. Major or severe symptoms were defined as unfamiliar symptoms, that make a person concerned whether they would pass by themselves or enhance over time, that may cause discomfort or pain up to severe pain, and may prevent a person from everyday activities. Such a scenario was invented due to the fact that during the pre-test, respondents required specification of the health problem to be able to make a choice. At the same time, during the pre-test, respondents stated that having some 


\section{Chapter 2}

minor symptoms would not be a reason for them to visit a physician and the choice tasks would not be meaningful to them.

Therefore, the current study is limited to cases where patients experience symptoms that can be described as major symptoms of an unfamiliar health problem (see Appendix C). The scenario implies that the symptoms are severe enough to force the patient to consult with a physician (i.e. the patient has already decided to visit a physician). Since the health problem described in the scenario is unfamiliar to the patient, he/she has no clear indication of which medical specialist is needed. Thus, visiting a general practitioner would be a rational option. Also, we have chosen for unfamiliar symptoms rather than a specific disease known to the respondent, because we aim to provide evidence for the improvement of physician services in Ukraine in general, and not for the improvement of physician services used by a specific group of patients. The pre-test indicated that the scenario is well understood and imaginable for the respondents. However, if the focus is on physician services provided to a specific group of patients, more concrete symptoms and diseases should be presented in the scenario.

\subsubsection{Data collection procedure}

At the development stage, the questionnaire was pre-tested on 55 respondents (a convenience sample). Data were collected in December 2009. Due to logistic reasons, the data collection was performed within a larger household survey carried out by the subcontractor (Kiev International Institute of Sociology). Therefore, we have no information about the nonresponse rate and, consequently, about the characteristics of non-respondents. For the sampling details, see Appendix A. The sample in our study included 303 respondents who agreed to participate in face-to-face structured interviews conducted by professional interviewers.

The sample was based on a three-stage random sampling procedure. First, 110 primary sampling units were randomly selected from the Ukrainian settlements (villages, towns, cities) with a probability proportional to the size of the settlement. Second, within those primary units, 207 voting precincts were randomly selected proportionally to the number of dwellings in the primary units. The voting precincts selected for the study comprised the secondary sampling units. Third, within each secondary sampling unit, 1-2 respondents were selected following a three-step procedure: 1) randomly selecting one dwelling; 2) listing and sorting all 
adults in this and consecutive dwellings by dwelling number, gender, and age ${ }^{1}$; 3 ) in case of 1 respondent per secondary sampling unit, recruiting the $3^{\text {rd }}$ adult in the sorting list, whilst in case of 2 interviews per secondary sampling unit, recruiting the $3^{\text {rd }}$ and $6^{\text {th }}$ adults in the list. The sample was intended to be representative for the adult population of Ukraine (aged 18 and older).

The questionnaire also included questions about socio-demographic characteristics such as age, gender, education, place of residence, current activities, health status, marital status, medical insurance, household income and composition. According to findings of Neuman and Neuman (2008), it was assumed that medical workers or people with medical education might express significantly different preferences due to their knowledge, background or professional belonging. Therefore, data on medical education were also collected to be able to test for the relevance of this characteristic.

\subsubsection{Statistical analysis}

A logistic regression was applied to analyze the choice of a physician. The underlying linear predictor of a choice was expressed as a function of the difference in utility $\left(\Delta U_{i}^{B-A}\right)$ that is expected to be obtained by a respondent $i$ when choosing physician B (alternative profile) over physician A (basic profile). This difference was expected to depend on differences in characteristics between physicians in a choice task $\left(\Delta X_{j}^{B-A}\right)$ and the interactions of these attribute differences with socio-demographic characteristics $\left(S_{n}\right)$.

$$
\Delta U_{i}^{B-A}=\sum_{j} \beta_{j} \Delta X_{j}^{B-A}+\sum_{j n} \gamma_{j n} \Delta X_{j}^{B-A} S_{n}
$$

The regression was tested for inclusion of random parameters and random intercept - both with a Gausian distribution. The model with the best statistical fit was used for further analysis.

The regression estimates were used to calculate the benefit (in monetary terms) gained by society with improvements of the characteristics, also called the "implicit price" or "part worth” of an attribute in monetary terms (Hanley et al., 2003; Lancsar et al., 2007; Ryan et al., 2001a), or marginal WTP for a marginal variation in an attribute. It was calculated as the

\footnotetext{
${ }^{1}$ Before selecting 'potential respondents', an interviewer enumerated and listed adult household members (i.e., 18 and older) living in a consecutive run of apartments (i.e., from lower to higher appartment numbers) until he or she had a list of occupants sorted by appartment, gender, and age.
} 


\section{Chapter 2}

marginal rate of substitution between improvement in the attribute and the increase in price which holds the utility level unchanged. In models with main effects only, this equation reduces to the ratio of the coefficient of a non-price attribute $\beta_{1}$ to the coefficient of the price attribute $\beta_{P}$. However, as discussed by Lancsar et al. (2007), inclusion of interactions will also imply the presence of socio-demographic characteristics $S_{n}$ that had significant interactions $\gamma$ with attributes under consideration in the partial derivatives. This was expressed as:

$$
M W T P=\left.\frac{\partial \Delta X_{1}}{\partial \Delta P}\right|_{\Delta U=0}=-\frac{\partial \Delta U / \partial \Delta X_{1}}{\partial \Delta U / \partial \Delta P}=-\frac{\beta_{1}+\sum_{n} \gamma_{1 n} S_{n}}{\beta_{P}+\sum_{n} \gamma_{P n} S_{n}}
$$

For marginal WTP calculations we held socio-demographic characteristics at their average sample level. Marginal WTP calculations should not be used as a presentation of potential fare levels, but only represent potential relative benefit gains/losses at the societal level.

\subsection{Results}

The descriptive statistics of socio-demographic characteristics that were included in the analysis are presented in Table A.1 in Appendix A. An overrepresentation of certain groups occurs, namely women aged between 30 and 69 and men between 50 and 59. In general men are underrepresented, especially in the age group between 60 and 69 years old. Also, the sample contains a large proportion of people with low income. About $72 \%$ of the respondents have a monthly income per person which is not higher than $1000 \mathrm{UAH}$ (approximately $€ 85$ per person at the survey time, with mean monthly income of approximately $€ 73$ per person). However, in DCE, socio-demographic features are included in the analysis through the interactions and thus, overrepresentations are accounted for. It is also to be noted that $6.6 \%$ of the respondents reported having a medical education (higher or secondary), even though not all of these respondents might work in the health care sector. Nevertheless, this group is large enough to include medical education as a variable in the analysis.

The results of the reduced regression model with the best fit are presented in Table 2.2. The model was checked for inclusion of interactions by likelihood ratio tests, and for inclusion of random intercept and random parameters by the Bayesian Information Criterion (BIC) (for a definition see Schwarz, 1978). The mixed-effect (random parameter) logit model with interaction terms shows the best fit for the data. 
Table 2.2 Results of DCE: reduced mixed effect logit model with significant ${ }^{\mathrm{a}}$ main effects and interactions

\begin{tabular}{|c|c|c|}
\hline \multirow[t]{2}{*}{ Effects } & Mean parameter estimate & $\begin{array}{c}\text { Random parameter } \\
\text { variation }\end{array}$ \\
\hline & Coeff. & Var (S.E.) \\
\hline \multicolumn{3}{|l|}{ Physician’s specialization } \\
\hline From GP to medical specialist & n.s. & \\
\hline \multicolumn{3}{|l|}{ State of the medical equipment } \\
\hline From outdated to modern & $2.205^{* * *}(0.192)$ & $0.886(0.172)$ \\
\hline \multicolumn{3}{|l|}{ Maintenance of the office } \\
\hline From old-looking to renovated & $0.672 * * *(0.172)$ & $0.319(0.318)$ \\
\hline \multicolumn{3}{|l|}{ Attitude of medical staff } \\
\hline From arrogant to polite & $3.210 * * *(0.230)$ & $0.933(0.175)$ \\
\hline \multicolumn{3}{|l|}{ Travel time to the office } \\
\hline 1 min additional & $-0.025 * * *(0.003)$ & $0.000(0.006)$ \\
\hline \multicolumn{3}{|l|}{ Waiting time in front of the office } \\
\hline 1 min additional & $-0.023 * * *(0.004)$ & $0.000(0.010)$ \\
\hline \multicolumn{3}{|l|}{ Size of patient payment ${ }^{\mathrm{a}}$} \\
\hline $1 \mathrm{UAH}^{\mathrm{a}}$ & $-0.031 * * *(0.003)$ & $0.026(0.003)$ \\
\hline \multicolumn{3}{|l|}{ Form of patient payment } \\
\hline From informal to formal & n.s. & \\
\hline Constant term & n.s. & $0.910(0.118)$ \\
\hline \multicolumn{3}{|l|}{ Physician’s specialization by: } \\
\hline younger than 20 & $1.236 * *(0.557)$ & \\
\hline assigned to any disability category & $-0.871 *(0.476)$ & \\
\hline missed more than 14 days due to illness & $0.620 * *(0.300)$ & \\
\hline high income: > 1000UAH per person & $0.552 * * *(0.185)$ & \\
\hline residing in Kyiv & $0.939 * *(0.412)$ & \\
\hline \multicolumn{3}{|l|}{ State of medical equipment by: } \\
\hline male & $-0.654 * * *(0.249)$ & \\
\hline no or primary education & $-0.608 *(0.323)$ & \\
\hline poor or very poor health & $-0.830 * *(0.344)$ & \\
\hline residing in small cities and towns & $0.885 * * *(0.326)$ & \\
\hline residing in Kyiv & $-1.277 * *(0.503)$ & \\
\hline \multicolumn{3}{|l|}{ Maintenance of the office by: } \\
\hline higher or doctoral education & $0.600 * * *(0.229)$ & \\
\hline 1 or 2 children in family & $0.463 *(0.239)$ & \\
\hline residing in rural areas & $0.716 * * *(0.218)$ & \\
\hline $30-80 \%$ of family members do not work & $-0.681 * * *(0.233)$ & \\
\hline \multicolumn{3}{|l|}{ Attitude of medical staff by: } \\
\hline older than 60 & $1.137 * *(0.515)$ & \\
\hline no or primary education & $-0.825 * *(0.366)$ & \\
\hline poor or very poor health & $-0.660 *(0.367)$ & \\
\hline residing in Kyiv & $-1.037 * *(0.514)$ & \\
\hline $30-80 \%$ of family members do not work & $-0.583 * *(0.251)$ & \\
\hline \multicolumn{3}{|l|}{ Travel time to the office by: } \\
\hline missed more than 14 days due to illness & $0.014 * *(0.007)$ & \\
\hline \multicolumn{3}{|l|}{ Waiting time in front of the office by: } \\
\hline not working & $0.017 * *(0.007)$ & \\
\hline missed more than 14 days due to illness & $0.026 * * *(0.009)$ & \\
\hline residing in middle cities & $-0.011 *(0.006)$ & \\
\hline \multicolumn{3}{|l|}{ Size of patient payment by: } \\
\hline older than 60 & $-0.023 * *(0.009)$ & \\
\hline very good or perfect health & $0.019 * *(0.008)$ & \\
\hline
\end{tabular}


Table 2.2 (continued) Results of DCE: reduced mixed effect logit model with significant ${ }^{\mathrm{a}}$ main effects and interactions

\begin{tabular}{|c|c|c|}
\hline \multirow[t]{2}{*}{ Effects } & Mean parameter estimate & $\begin{array}{c}\text { Random parameter } \\
\text { variation }\end{array}$ \\
\hline & Coeff. & Var $\quad$ (S.E.) \\
\hline \multicolumn{3}{|l|}{ Form of payment by: } \\
\hline younger than 20 & $-1.107 *(0.582)$ & \\
\hline no missed days due to illness & $0.605^{* * *}(0.170)$ & \\
\hline high income: > 1000UAH per person & $-0.385 * *(0.189)$ & \\
\hline residing in rural areas & $-0.439 * *(0.214)$ & \\
\hline residing in small cities and towns & $-0.612 * *(0.269)$ & \\
\hline \multicolumn{3}{|l|}{ Model statistics } \\
\hline Number of observations & 3189 & \\
\hline Number of groups & 220 & \\
\hline Likelihood ratio test (chi2) & $301.42 * * *$ & \\
\hline Wald test (chi2) & $436.97 * * *$ & \\
\hline Log-likelihood & -1440.99 & \\
\hline AIC & 2967.99 & \\
\hline BIC & 3228.89 & \\
\hline
\end{tabular}

For all models, including the interim estimations that are not presented, the constant term is highly insignificant, which indicates that respondents did not have any specific preference for the profiles per se and made choices based on the levels of attributes presented in the profiles. This is a positive fact because profiles in this study were designed so that they varied between choices and covered all meaningful alternatives.

Six attributes signifying the 'state of medical equipment', 'maintenance of a physician's office', 'treatment of medical staff', 'travel time', 'waiting time’ and 'patient payment' have statistically significant main effects with theoretically valid directions of relations (the signs of the coefficients). Attributes describing 'physician's specialization' and 'type of payment' have significant effects on the preferences in certain socio-demographic groups only.

Specialization of a physician does not significantly affect choices at the sample level. However, belonging to any disability category shifts preferences towards general practitioner. Capital residence and higher income are found to shift preferences towards medical specialist, and these two characteristics are associated (odds ratio 4.27, SE 2.51). Hence, it can be concluded that a higher socio-economic status represented by a higher income, and residence in the capital Kyiv is associated with a preference for a medical specialist over a general 
practitioner. Also people younger than 20 and those who missed more than 14 days due to illness in the past year are more likely to prefer a medical specialist.

The results indicate an overall significant preference (at the sample level) for modern equipment and renovated premises. As for the group differences for the state of medical equipment attribute it is observed that among men, people with no or primary education only, poor and very poor health, and residing in capital this preference diminishes, while for people residing in small cities or towns it increases. Well maintained premises is a more important choice factor for people with higher or doctoral education, having 1 or 2 children in a household, residing in rural areas, and it is less important for respondents from households with $30-80 \%$ of members not working.

The attribute 'attitude of medical staff' being the most valued factor at the sample level, is of higher importance to older people, and of less importance to low educated people, those with poor or very poor health, residing in Kyiv, and living in households with 30-80\% of members not working.

The attributes 'travel time' and 'waiting time' have the expected negative coefficients. This indicates that respondents prefer a shorter travel and waiting time. Regression coefficients indicate that travel and waiting time are less important for people that missed many days due to illness in the past year. People who are not working (but are not retired) value waiting time less, while people residing in middle sized cities place more value on waiting time reduction.

Table 2.3 Marginal WTP for a total change in quality and access attributes

\begin{tabular}{llc}
\hline Attribute & Total attribute change & $\begin{array}{c}\text { Marginal WTP, } \\
\text { UAH }{ }^{\text {a }} \text { per visit }\end{array}$ \\
\hline $\begin{array}{l}\text { Physician's specialization } \\
\text { State of the medical equipment }\end{array}$ & From GP to medical specialist & 8.93 \\
Maintenance of the office & From outdated to modern & 57.43 \\
$\begin{array}{l}\text { Attitude of the medical staff } \\
\text { Travel time to the office }\end{array}$ & From arrogant to polite & 27.26 \\
$\begin{array}{l}\text { Waiting time in front of the } \\
\text { office }\end{array}$ & From 60 min to 15 min & 86.68 \\
Form of payment & From informal to formal & 32.91 \\
\hline
\end{tabular}

${ }^{a}$ Average daily exchange rate during the period of data collection is $11.5424 \mathrm{UAH} / €$ 


\section{Chapter 2}

The price attribute, i.e. 'size of patient payment' has a negative main effect as expected, which means that patients prefer paying less to paying more. The model shows that elderly people are even more sensitive to a price increase. On the other hand, people with good or perfect health are less sensitive to the price attribute.

Though 'form of payment' did not demonstrate a significant impact as a main effect, its effects appear significant in some groups. The preference for paying formally is found for those who have no missing days due to illness in the past year. Groups that rather prefer paying informally are younger people, the high income group, residing in rural areas and in small cities or towns.

Table 2.3 represents the estimates of the marginal WTP for the total variation change in the main attributes of the physician services calculated as the MRS. These values cannot be considered as a notion of possible levels of patient payments, but they only show the monetary value that Ukrainian consumers place on the quality and access attributes, namely their change from the worst to the best state presented in choice tasks.

As indicated in Table 2.3, respondents place a value of 57.43 UAH for each visit to a physician where modern medical equipment is available instead of outdated equipment. The value of the change from fully old-looking to renovated premises is $27.26 \mathrm{UAH}$ per visit. The monetary value of the change in attitude of medical staff is the highest: $86.68 \mathrm{UAH}$ per visit to a physician. For travel and waiting time, the monetary value of a reduction of 45 minutes travel time and 35 minutes waiting time is $32.91 \mathrm{UAH}$ and $22.80 \mathrm{UAH}$ per visit respectively. Changes in physician's specialization and form of payment have a very low overall monetary value (8.93 UAH and 1.68 UAH respectively) as they appear to be significant predictors of choice only in some groups, indicating the minor importance attached to these attributes by respondents.

\subsection{Discussion and conclusion}

To make our DCE feasible, we limited the number of attributes and attribute levels. We recognize that the inclusion of other attributes and more levels could provide further detail on the preferences of Ukrainian consumers for physician services. Nevertheless, our results provide information about the relative importance of the attributes in our study, which is still relevant to policy-making. Our analysis is also limited to the scenario of major or severe 
symptoms that are unfamiliar to the patient. Therefore, our results may not hold for the case of minor and familiar symptoms or a detailed specification of a health problem. However, the impact of a certain health problem on preferences is beyond the scope of this article which aims to study preferences in general. We also analyze only binary choices between two profiles (fixed and alternative) without the possibility to opt out. Therefore, the WTP estimates might be inflated. Including an opt-out alternative may give more valid estimates because in reality patients may forgo visiting a physician despite the severe symptoms, e.g. due to an unaffordable service price. Finally, there is a slight overrepresentation of certain age and gender groups in our sample. However, the impact of these and other socio-demographic factors was controlled by their inclusion in the models with interaction. A model with interaction is used to present and discuss the main findings of the study.

\subsubsection{Comparison of the findings with similar studies}

Our results for the marginal WTP should not be interpreted as the actual amount of patient fees but as a relative monetary value of a benefit. Unfortunately, there are no published results from previous research on WTP for physician services in Ukraine. Thus, we can only compare with the results of studies carried out in other countries. The comparison of the magnitude of the economic values across studies from different countries due to contextual differences can be done only in relative terms, i.e. in relation to average household income. The most valued attribute in our study: the change in attitude from arrogant to polite, amounts to $3.69 \%$ of the average household income (about $2347.32 \mathrm{UAH}$ per household). This is, however, slightly higher than, but comparable to other DCE studies from the UK (CheraghiSohi et al., 2008), Sweden (Hjelmgren and Anell, 2007) (for national statistics on income see SCB Statistics Sweden, 2010), where the most important attributes are valued at 3\% and 2.5\% of household income respectively. This implies that, first of all, the monetary benefit estimates are plausible, and, secondly, that organizational reforms should take place as the Ukrainian population highly values the proposed quality improvements.

The structure of preferences implied by our findings differs from the studies mentioned above, which might be due to the different design of DCE, the attribute choice and construction of attribute levels. Our results are, however, consistent with those from other studies where the importance of interpersonal aspects of care (e.g. Vick and Scott, 1998; Coulter, 2005; Leon, 2003) is shown. In the systematic literature review by Wensing et al. 
(1998), interpersonal aspects of care were ranked higher than technical aspects by the percentage of studies reporting their importance.

It should be noted that the difference in preference structures in our study and in previous research could be also due to contextual differences since these studies are carried out in Western European countries, where health care systems and socio-economic context are rather different than those in Ukraine. A study from Bulgaria (Pavlova et al., 2004) based on rating conjoint method, analyzes a similar set of physician services attributes but without interpersonal aspects. In general, the structure of preferences is similar to what is found in our study: clinical quality represented by professional reputation and medical equipment is followed by facility maintenance and time costs for the visit.

\subsubsection{The preference for quality and access related attributes in Ukraine}

Thus, among the quality and access characteristics which define the choice of the physician, attitude of the staff is the most important to Ukrainian consumers. This is, however, a broader concept which also includes the attention devoted to the patient, level of information, and involvement in care. It is important to further operationalize and study preferences for these different aspects of health care in Ukraine. The second most important attribute is the state of medical equipment, which represents the clinical efficiency and safety of care in a scenario of major or severe symptoms. Improvements in waiting and traveling time, as well as in the maintenance of the physician office are still important, though they would bring much less benefit than the improvements in the interpersonal aspects and clinical quality.

Taking into account the structure of preferences, the general recommendation is that in primary care, the priority should be placed on the development of the skills of medical personnel. This especially relates to the training of the interpersonal aspects of care, such as communication with the patient, which is now frequently unsatisfactory. The development of an infrastructure (a network of facilities) is a secondary task with a much lower priority.

Elderly people value the attitude of the medical staff higher than others. This also indicates that they lack it the most. In general, the Ukrainian system is ill adapted to meet the needs of elderly people, and least of all in the rural areas (Bezrukov, 2003). Care for elderly is only regarded within the framework of geriatrics which is not separated from the medical system, whilst long-term care is absent as a category in Ukraine. Special attention should be placed on the needs of elderly and training of medical staff in addressing those needs. 
Place of residence also affects the preference for quality characteristics of physician services included in our study. In particular, dwellers of the capital city place less importance on the attitude of the medical staff and the state of medical equipment, while rural and small town dwellers value higher medical equipment and maintenance of the office. Nevertheless, the relative importance of the characteristics in these groups does not differ from those in the general population. Such patterns might be explained by substantial territorial disparities in the quality of the health care (state of the facilities, access to care), rural areas being much worse-off, especially compared to the capital (Ukrainian Helsinki Human Rights Union, 2012). This calls for measures to improve the situation in those areas to bring it up to standards.

People with a lower social (education), economic (earning members of the family) or health status appear to value the quality characteristics slightly less (i.e. excluding time costs). However, the overall preference structure in these groups does not differ dramatically from that of the general population. Our results suggest that people, who are likely to use health care services, i.e. have poor health status and elderly, place higher importance on the price levels in their choice of a physician than healthier and younger individuals. For people with poor health, this also implies paying less attention to the quality and access characteristics. Naturally, the welfare of people, who have to use these services more often, is more affected by the price of these services, compared to healthy individuals. Hence, we may expect then unhealthy people to attach more weight to the price of service. Therefore, the observed heterogeneity of the price weight can be interpreted as a sign of the theoretical validity of the DCE. It also highlights the importance of protecting frequent users, such as elderly and people with chronic conditions, from overspending on health care. Currently, Ukrainian patients spend rather substantial amounts out-of-pocket via informal and quasi-formal payments which represent a financial burden to the households, especially to low-income and sociallydisadvantaged groups (Gryga et al., 2010).

\subsubsection{Trade-off between formal and informal payments}

Our results demonstrate that non-frequent users (healthier individuals), who are rarely confronted with the health care system, prefer formal ways of payment. However, those who have to use health services frequently (unhealthier individuals) are more tolerant towards informal payments. This is expressed by their indifference towards this form of payment. Thus, tolerance of informal payments seems to be the result of the interaction between patient 
and provider due to shortcomings in the Ukrainian health system rather than a part of patients' preferences.

The risk groups who might explicitly prefer paying informally are people from rural areas and small towns, higher income groups and youth (under 20 years). In rural areas, this might be related to the substantial lack of medical personnel (Ukrainian Helsinki Human Rights Union, 2012) which impedes access to services by making patients pay for the physician's attention. Higher income group and youth might prefer informal payments for the sake of quicker access and personalized treatment. These preferences indirectly indicate a failure to provide access to the services of a satisfactory quality level, especially in rural areas.

Thus, our results suggest that Ukrainian patients do not intrinsically prefer informal payment channels but make use of them due to barriers to access and/or the absence of formal charges. Given the adverse effects of informal payments on equity in the health care system and its sustainability, measures for their eradication are necessary. General anticorruption measures in combination with public campaigns have proved to be effective in some Eastern European countries, for example Poland (Stepurko et al, 2013; Golinowska, 2010). In addition to this, some Asian countries report successful cases of formalization of informal payments (Baschieri and Falkingham, 2006; Barber et al., 2004). The choice of specific measures should match the broader policy aims.

\subsubsection{Role of gate-keeping}

In our study, we find no clear preference for the type of physician (general practitioner or specialist). This could have both positive and negative consequences for policy making. The positive aspect is that there is no motivation to avoid primary level contact and to go directly to higher and more costly levels of health care. The negative side is a potential for resistance among the part of the population that prefers direct referral. From this perspective it is important to design interventions that facilitate primary physician contacts and prevent direct access to costly specialized services, thus, strengthening a gate-keeping role of general practitioners. This can include introducing penalizing fees for direct referrals.

Potentially the group preferring direct referral consists of people with a higher socioeconomic status, which may be due to their life style, higher level of knowledge, and higher value of time. It also includes younger people and "experienced" - those who missed a lot of days due illness. In the study by González (2010), it is suggested that patients with symptoms that are chronic, inherited, recurrent or easily recognizable possess more accurate information 
about their health problem and should be given free choice of medical provider. In any case, caution should be taken in recognizing whether patient self-awareness is correct as most patients do not have medical knowledge. On the other hand, people assigned to any disability category already demonstrate a preference for visiting a general practitioner.

\subsubsection{Overall policy implications and conclusions}

In our study, we have aimed to contribute to the policy discussion about the design of health care reforms in Ukraine by providing evidence on consumer preferences for physician services. We conclude that patients place a high value on quality and access improvements. Priority should be given to the improvements in interpersonal aspects of care followed by medical safety and quality. This may be achieved by improving the training of medical staff with a special focus on communication with the patient. Investments in facilities and their networks are of lower priority, though still important.

Measures to improve quality of care for all population groups and residential areas are necessary. Especially, rural areas and small towns lack good quality services (MOH, 2010). Higher quality standards and greater equality may also be seen as a tool to diminish the widely spread informal payments.

The intention of some population groups to bypass the general practitioner is slight and, thus could be efficiently overridden by the introduction of price disincentives for direct access to a medical specialist. The policies of tackling informal payments might meet obstacles in the form of inertial involvement into them. Special attention should be paid to risk groups that explicitly prefer paying informally, i.e. people living in rural areas or small towns, people with higher income and youth. 



\section{CHAPTER 3}

Willingness to pay for physician services at a primary contact in Ukraine: Results of a contingent valuation study

Based upon:

Danyliv, A., Pavlova, M., Gryga, I., \& Groot, W. (2013). Willingness to pay for physician services at a primary contact in Ukraine: results of a contingent valuation study. BMC Health Services Research, 13(1), 208. DOI: 10.1186/1472-6963-13-208 


\section{Abstract}

Background: The existence of quasi-formal and informal payments in the Ukrainian health care system jeopardizes equity and creates barriers to access to proper care. Patient payment policies that better match patient preferences are necessary.

Objective: We analyze the potential and feasibility of official patient charges for public health care services in Ukraine by studying the patterns of fee acceptability, ability and WTP for public health care among population groups.

Methods: We used CV data collected in a small-scale survey of 303 respondents representative of the adult Ukrainian population. Three decision points were separated: objection to pay, inability to pay, and level of positive non-zero WTP. These decisions were studied for relations with quality profiles of the services, and socio-demographic characteristics of the respondents and their households.

Results: The likelihood to object to pay is mostly determined by the quality characteristics of the services. Objection to pay is not related to corresponding behavior in real life. The likelihood of being unable to pay is associated with older age, lower income, and a larger share of household members with now income. The level of positive WTP is positively related to income ( $+7 \%$ per $1000 \mathrm{UAH}$ increase in income) and is lower for people who visited a doctor but did not pay (-22\%).

Conclusions: Rather substantial WTP levels (between $0.9 \%$ and $1.9 \%$ of household income) for one visit to physician indicate a potential for official patient charges in Ukraine. User fees may cover a substantial share of personnel cost in the outpatient sector. The patterns of inability to pay support well designed exemption criteria based on age, income, and other aspects of economic status. The WTP patterns highlight the necessity for payments that are proportional to income. Other methodological and policy implications are discussed. 


\subsection{Introduction}

The Constitution of Ukraine guarantees free of charge provision of medical aid in public facilities for every citizen (Ukraïna, 1996). Thus, it practically bans all patient charges in the public sector. However, there is a mismatch between regulation and reality. This is caused by the quasi-formal charges (providers' requests to pay official charitable contributions to the health care organization which should be voluntary in nature). There are also informal (underthe-table) charges in the form of cash or in-kind gifts paid to health care providers for better services. Such unregulated charges have distortive effects on health care provision and consumption (Gryga et al., 2010; State Statistical Service of Ukraine, 2010b; Lekhan et al., 2004). Most important, they jeopardize equity in health care and create barriers to access even in the absence of official patient charges.

This 'status quo' inherited from the Semashko system established in Ukraine during the Soviet Union period, seems to currently satisfy only policy-makers who, as suggested by anecdotal evidence, receive health care abroad or in the best public facilities (TSN, 2011). The political establishment guards zealously the tax-funded free-of-charge principle of public health care provision for the sake of retaining electoral support. However, this policy conservatism is at odds with the inefficiency of resource allocation and the high level of patient dissatisfaction with the health care system (Gorshenin Institute, 2011). Ukrainian patients suffer from a lack of access to proper care (especially in rural areas) (Ministry of Health of Ukraine, 2010), long waiting lines, the reluctance of unmotivated medical staff to offer adequate care (Gryga et al., 2010), and obsolete and inefficient treatment methods (Committee on Economic Reforms, 2010). They are left with the option to seek better access and quality by means of quasi-formal and informal payments, unless they can use their personal connections to obtain the services they desire (Gryga et al., 2010 ; Pavlova et al., 2011).

The data suggest that patients in Ukraine cover a substantial part of health care expenditure out of pocket. WHO data indicate that private out-of-pocket expenditure in Ukraine amounts to around $40.5 \%$ of total health expenditures (WHO, 2012) with a very small part being administered in the private sector (State Statistical Service of Ukraine, 2010b) although the exact size of out-of-pocket payments in the private sector is unknown. This is one of the largest shares of out-of-pocket payments in Europe which contradicts the official 'free-ofcharge’ policy in Ukraine. 
Most of the out-of-pocket spending is for medical goods. These should be provided to patients for free in public health care settings, but frequently patients have to purchase them outside the setting due to the absence of these medical goods in these facilities (see data at WHO, 2012). In addition to this, evidence suggests that the share of unofficial patient charges (i.e. quasi-formal and informal charges) at public health care settings is substantial (Pavlova et al., 2011; Balbanova et al., 2004; Litvak et al., 2001). Unregulated patient charges create a considerable financial burden on households and provoke reduced and unequal access to public health care services in Ukraine (Gryga et al., 2010). This problem is recognized at the national level (e.g. Ministry of Health of Ukraine, 2010; Committee on Economic Reforms, 2010). In view of this, it is not surprising that about $50 \%$ of the Ukrainian patients state that they either have to borrow money to pay for health care or to forgo services due to patient charges (Pavlova et al., 2011).

Obviously, there is a need for a more efficient patient payment policy. Together with other anticorruption measures, official patient charges may to a certain extent reduce the need to pay unofficial charges. They may provide extra revenues for health care providers, reduce inefficient health care provision and excess demand for health care (Pavlova et al., 2010). However, the implementation of official patient charges is not straightforward. Apart from the need to establish a legal base for the implementation of such charges, the exact patient payment mechanism should be carefully designed and account for the population's needs and ability to pay.

In this study, we analyze the potential and feasibility of official patient charges for public health care services in Ukraine. We address this issue by studying the patterns of fee acceptability, ability and WTP for public health care across population groups. The analysis is based on CV data from a household survey among a small representative sample of the Ukrainian population. CV is a SP method that is widely applied in health services research to study individual WTP for a health care good (for a review see e.g. Diener et al., 1998; Klose, 1999; Smith and Sach, 2010).

We focus on fees for physician services provided to patients with major health problems. We relate acceptability, ability and WTP stated by the respondents to the specialization of the physician and to the quality/access profile of the physician. This is relevant for policy making because the role of primary care is still neglected in Ukraine as primary care providers are often bypassed by patients and the number of general practitioners (GPs) is still insufficient. At the same time, the quality of and access to care is reported to be unsatisfactory (Gorshenin 
Institute, 2011), which is frequently explained by the severe under-financing of the system (Karamyshev et al., 2006; Vynogradov, 2007). The unregulated patient payments are known to fill this financial gap. The question is whether Ukrainian patients are willing and able to pay officially for quality and access improvements. Our study can be a useful starting point for policy discussions in Ukraine as well as in other countries that face similar problems (especially other former socialist countries).

\subsection{Methods}

In this chapter, we used CV data from the same small-scale survey from Ukraine as in Chapter 2. The sample consisted of 303 respondents who agreed to participate in face-to-face interviews conducted in December 2009. It was representative of the adult Ukrainian (aged 18 and older) population. Respondents were asked to state if they would be willing to pay for a visit to a physician with quality and access characteristics expressed by four profiles. The scenario of major symptoms was selected as during the pre-test it was found that the respondents required some specification of the health condition to be able to say something about their willingness to pay for treatment. On the other hand, this scenario allowed keeping the broad focus on physician services without narrowing down to a specific disease.

In case a respondent was willing to pay for a given physician profile, a combination of payment cards and open-ended questions was applied. First, the respondent selected a payment interval from the card, then, an exact amount within the interval (open-ended question). Payment cards enabled framing respondents' answers (preventing overstatement), while exact values elicited by the open-ended questions served as a more precise indication of the maximum WTP level. As discussed in section 1.2, open-ended valuation, apart from providing more precise information, is also expected to provide more conservative estimates which we prefer in the policy analysis of patient charges. If a respondent was not willing to pay, s/he was asked to state the reason. The exact wording of the CV tasks is presented in Appendix C.

The four valuation profiles were designed in a way to estimate two separate effects on WTP for physician services: (i) the effect of a physician's specialization (general practitioner or medical specialist), and (ii) the effect of quality/access improvements (namely the joint effect of improvements in the state of medical equipment, maintenance of the physician office, and 


\section{Chapter 3}

the reduction in waiting time in front of the physician's office from 45 to 10 minutes). The four valuation tasks and the effects on WTP studied are summarized in Table 3.1.

The socio-demographic and household characteristics are summarized in Table A.1 in Appendix A. The sample does not differ substantially from the entire Ukrainian population except for a slight over-presentation of women and some specific age groups. To account for this slight overrepresentation, we included these and other socio-demographic characteristics in the analysis.

Table 3.1 Physician profiles included in the CV tasks and the effects on WTP studied

\begin{tabular}{|c|c|c|c|c|c|}
\hline \multirow[b]{2}{*}{ Attributes $^{\text {a }}$} & \multicolumn{4}{|c|}{ Physician profiles included in the CV tasks } & \multirow[b]{2}{*}{$\begin{array}{l}\text { Effects } \\
\text { on WTP }\end{array}$} \\
\hline & $\begin{array}{c}\text { GP } \\
\text { basic profile }\end{array}$ & $\begin{array}{c}\text { GP } \\
\text { improved } \\
\text { profile } \\
\end{array}$ & $\begin{array}{c}\text { Specialist } \\
\text { basic profile }\end{array}$ & $\begin{array}{c}\text { Specialist } \\
\text { improved } \\
\text { profile }\end{array}$ & \\
\hline $\begin{array}{l}\text { Physician’s } \\
\text { specialization }\end{array}$ & $\begin{array}{l}0= \\
\mathrm{GP}\end{array}$ & $\begin{array}{l}0= \\
\text { GP }\end{array}$ & $\begin{array}{c}1= \\
\text { Specialist }\end{array}$ & $\begin{array}{c}1= \\
\text { Specialist }\end{array}$ & Specialization \\
\hline $\begin{array}{l}\text { State of the medical } \\
\text { equipment }\end{array}$ & $\begin{array}{c}0= \\
\text { Outdated }\end{array}$ & $\begin{array}{c}1= \\
\text { Modern }\end{array}$ & $\begin{array}{c}0= \\
\text { Outdated }\end{array}$ & $\begin{array}{c}1= \\
\text { Modern }\end{array}$ & \\
\hline $\begin{array}{l}\text { Maintenance of } \\
\text { the physician's office }\end{array}$ & $\begin{array}{l}0= \\
\text { Old-looking }\end{array}$ & $\begin{array}{c}1= \\
\text { Renovated }\end{array}$ & $\begin{array}{l}0= \\
\text { Old-looking }\end{array}$ & $\begin{array}{c}1= \\
\text { Renovated }\end{array}$ & $\begin{array}{l}\text { Quality/access } \\
\text { improvements }\end{array}$ \\
\hline $\begin{array}{l}\text { Waiting time in front } \\
\text { of the office }\end{array}$ & $45 \min$ & $10 \mathrm{~min}$ & $45 \mathrm{~min}$ & $10 \mathrm{~min}$ & \\
\hline
\end{tabular}

${ }^{\text {a }}$ Two attributes remained constant in all profiles: attitude of the medical staff = polite, and travel time to the physician's office $=15$ min.

We calculated the proportion of respondents who were willing and unwilling to pay for physician services (with a specified reason for their unwillingness to pay - object to pay and/or inability to pay). We also estimated the mean WTP amount. For the purpose of comparison, we looked at three measures of mean WTP: (i) including all answers (both positive and zero WTP answers), (ii) excluding protest answers (i.e. statements of unwillingness to pay due to an objection to pay fees), and (iii) including only positive WTP values. From a methodology point of view, protest answers should be excluded from the analysis since those who just object to pay may not really place a zero value on the services under valuation and would be willing to pay in a real market (Boyle, 2003). It should be noted however that this issue is still debated. 
We used a three-phase modeling approach because the WTP data had been obtained using a sequence of three main questions (see Figure 3.1): first whether the respondent is willing to pay (positive or zero WTP), and second, given a positive WTP, what is the maximum WTP level, or given a zero WTP, what is the reason for the unwillingness to pay. Three main categories were used to structure the reasons for the unwillingness to pay: objection to pay, inability to pay or both. Hence, respondents who selected the last category were assigned as being both unable and objecting to pay.

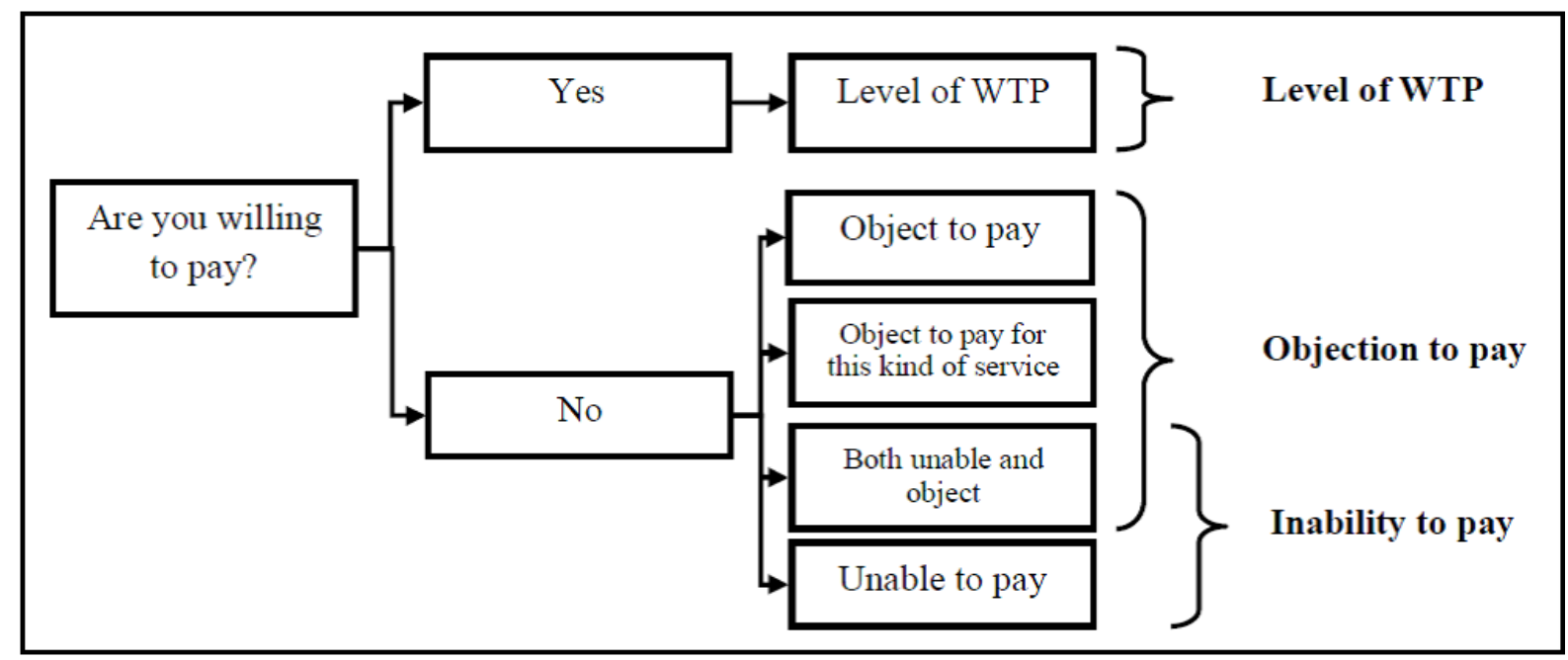

Figure 3.1. Decision sequence in the CV tasks and resulting modeling points

Thus, as presented in Figure 3.1, three decisions were separated: objection to pay, inability to pay, and the level of positive non-zero WTP. For the first model (objection to pay), the cases $(y=1)$ include those who stated that they "object to pay", "object to pay for this kind of service", or "both unable and object to pay". They were compared to those who do not object $(y=0)$, i.e. stated a zero WTP due to the inability to pay only, or a positive WTP. The second model (inability to pay) included those who stated that they either are "unable to pay" or "both unable and object" as cases $(y=1)$. They were compared to those who are not unable to pay $(y=0)$, i.e. stated a zero WTP due to objection only or a positive WTP. These two models have binary response variables; hence, we use random effect logistic regression (considering that one respondent evaluates four profiles). Denote the probability of a positive response (object to pay in the first model or being unable to pay in the second) for a respondent $i$ valuing profile $j$ as $P_{i j, y=1}$. Then, the log odds can be described as a linear combination of the 


\section{Chapter 3}

socio-demographic characteristics of the respondent and characteristics of the physician profile under valuation:

$$
\log \left(\frac{P_{i j, y=1}}{P_{i j, y=0}}\right)=\log \left(\frac{P_{i j, y=1}}{1-P_{i j, y=1}}\right)=\beta_{X} X_{j}+\beta_{S D C} \cdot S D C_{i}+\beta_{0}+\varepsilon_{i j}+v_{i},
$$

where $X_{j}$ is a set of profile $\mathrm{j}$ specific characteristics, $S D C_{i}$ are socio-demographic characteristics of the respondent $i, \varepsilon_{i j}$ is a stochastic error term and $v_{i}$ is a respondent specific random element. In this specification exponential coefficients represent the odds ratios for the change in characteristics.

$$
\exp \left(\beta_{x^{*}} \partial x\right)=\frac{p\left(Y \mid x^{*}+\partial x\right) /\left(1-p\left(Y \mid x^{*}+\partial x\right)\right)}{p\left(Y \mid x^{*}\right) /\left(1-p\left(Y \mid x^{*}\right)\right)}
$$

The third model (level of WTP) included only positive WTP levels. However, the positive WTP distribution was found to be skewed compared to the normal distribution. Therefore, in the analysis, we used a logarithmic transformation of positive WTP, which better resembled a normal distribution. The logarithm of respondent's $i$ WTP for a visit to a physician with profile $j$ can be specified as random effect linear regression (considering that one respondent evaluated 4 profiles):

$$
\log \left(W T P_{i j}\right)=\beta_{X} X_{j}+\beta_{S D C} \cdot S D C_{i}+\beta_{0}+\varepsilon_{i j}+v_{i} .
$$

In this specification, coefficients represent percentage changes in WTP in response to a unit change in the characteristics:

$$
\beta_{X, S D C}=\partial \log \left(W T P_{i j}\right) / \partial X, S D C={ }^{\partial W T P_{i j}} /\left(W T P_{i j} \cdot \partial X, S D C\right)(4)
$$

A set of socio-demographic characteristics (see Table A.1 in Appendix A) was included in the three models, as well as an indicator of the physician's specialization and an indicator of service improvement. The models were also checked for the inclusion of interactions between service characteristics and socio-demographic characteristics but this did not add to the model fit (hence, it reduced the model performance in terms of the Bayesian Information Criterion and degrees of freedom). Therefore, interactions were not included in the final models. The models were reduced to only statistically significant variables in order to see which factors had a stable effect. Both full and reduced versions are presented and discussed. 


\subsection{Results}

As displayed in Figure 3.2, a substantial part of the sample is willing to pay official fees for physician services. The share of respondents willing to pay is considerably higher (about three times higher) for physician profiles that indicate quality/access improvements than for the corresponding basic profiles. Specialization itself does not seem to have a large effect on the proportions of those who are willing or unwilling to pay. For profiles with less attractive quality/access characteristics, the dominant reason for being unwilling to pay is that people object to pay for such services. In contrast, profiles with better quality/access characteristics practically do not state this as a reason for their unwillingness to pay. Other reasons for unwillingness to pay (i.e. cannot afford, object to pay for medical services, and both) are stated relatively infrequently. However, $11-20 \%$ of the respondents say that they are unable to pay (unable or both unable and object).

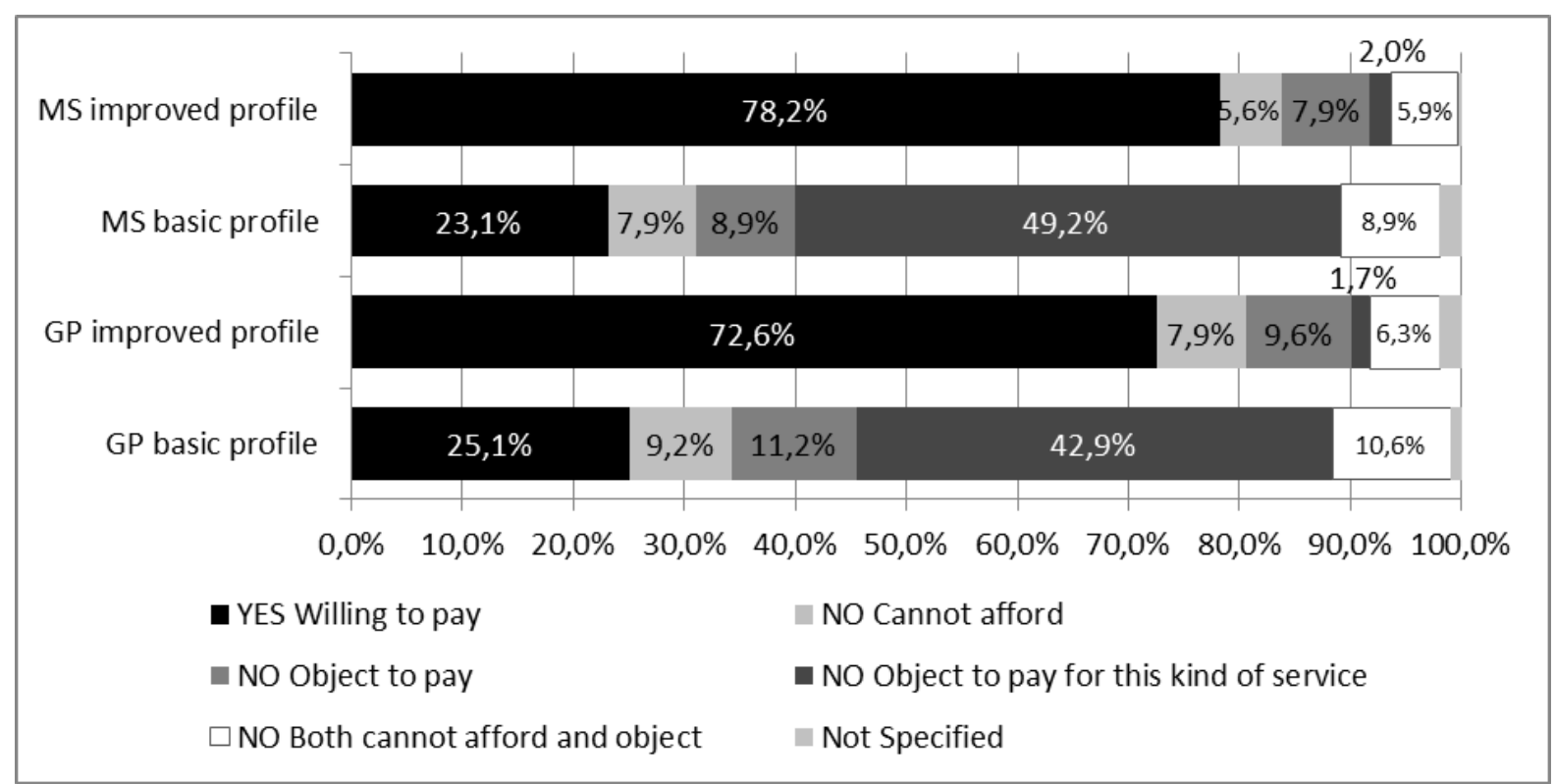

Figure 3.2. Proportion of respondents willing/unwilling to pay for physician services

Table 3.2 presents the three estimates of the mean WTP for physician services: (i) when all answers are considered (both positive and zero WTP answers), (ii) when only non-protest answers (excluding those who object to pay) are considered, and (iii) when only positive WTP answers are considered. Regardless of the type of estimate, the mean WTP is significantly higher for both a GP and a medical specialist with better characteristics than for physicians with less attractive characteristics. Due to the large number of protest zeros for the less 


\section{Chapter 3}

attractive profiles, the differences between the two mean estimates that include zero WTP answers (with and without protest answers) is substantial: 9.36 versus 20.39 UAH per visit for a GP (around $€ 0.81$ and $€ 1.80$ respectively), and 8.91 versus $22.10 \mathrm{UAH}$ per visit for a medical specialist (around $€ 0.77$ and $€ 1.91$ respectively). However, for the profiles with more attractive characteristics the difference between these two means is not so large (around $5 \mathrm{UAH}$ for both specialization modes). Specialization itself appears to have a minor impact on mean WTP although in most cases, WTP for a medical specialist is higher than that for GP services.

Table 3.2 Mean WTP for physician services

\begin{tabular}{lcccccr}
\hline \multirow{2}{*}{ Profile } & \multicolumn{5}{c}{ Willingness to pay, UAH $^{\mathbf{a}}$} \\
\cline { 2 - 7 } & $\begin{array}{l}\text { All answers } \\
\text { included } \\
\text { Mean (S.D.) }\end{array}$ & $\mathrm{N}$ & $\begin{array}{l}\text { Objection answers } \\
\text { excluded } \\
\text { Mean (S.D.) }\end{array}$ & $\begin{array}{l}\text { Only positive } \\
\text { WTP included } \\
\text { Mean (S.D.) }\end{array}$ & N \\
\hline GP basic profile & $9.36(23.47)$ & 302 & $20.79(31.44)$ & 136 & $37.21(34.06)$ & 76 \\
GP improved profile & $32.59(45.12)$ & 297 & $37.37(46.43)$ & 259 & $44.81(47.46)$ & 216 \\
Specialist basic profile & $8.91(23.13)$ & 300 & $22.10(32.23)$ & 121 & $38.20(34.39)$ & 70 \\
Specialist improved profile & $40.50(48.92)$ & 301 & $45.14(49.58)$ & 270 & $51.87(49.75)$ & 235 \\
\hline
\end{tabular}

${ }^{\mathrm{a}}$ Average daily exchange rate during the period of data collection is $11.5424 \mathrm{UAH} / €$

Table 3.3 presents the results of the three modeling processes (as described in the previous section): objection to pay, inability to pay, and level of positive WTP. Each of the modeling stages contains two versions of the model: a full model with all predictors, and a reduced model with only significant predictors. Further, we only discuss conclusions about predictors that are insensitive to the specification of the model, i.e. significant in both the full and reduced models.

Objection to pay for physician services is found to be strongly related to the quality/access characteristics of the profile. As can be seen from the significant negative coefficients, profiles with better quality/access characteristics have lower odds (80-100 lower chances) to object to pay. This is in line with the finding above that those who object to pay for physician services with less attractive quality/access characteristics constitute the larger share of the respondents who are unwilling to pay. 
Table 3.3 Results of modeling WTP: objection to pay, inability to pay, and level of positive WTP

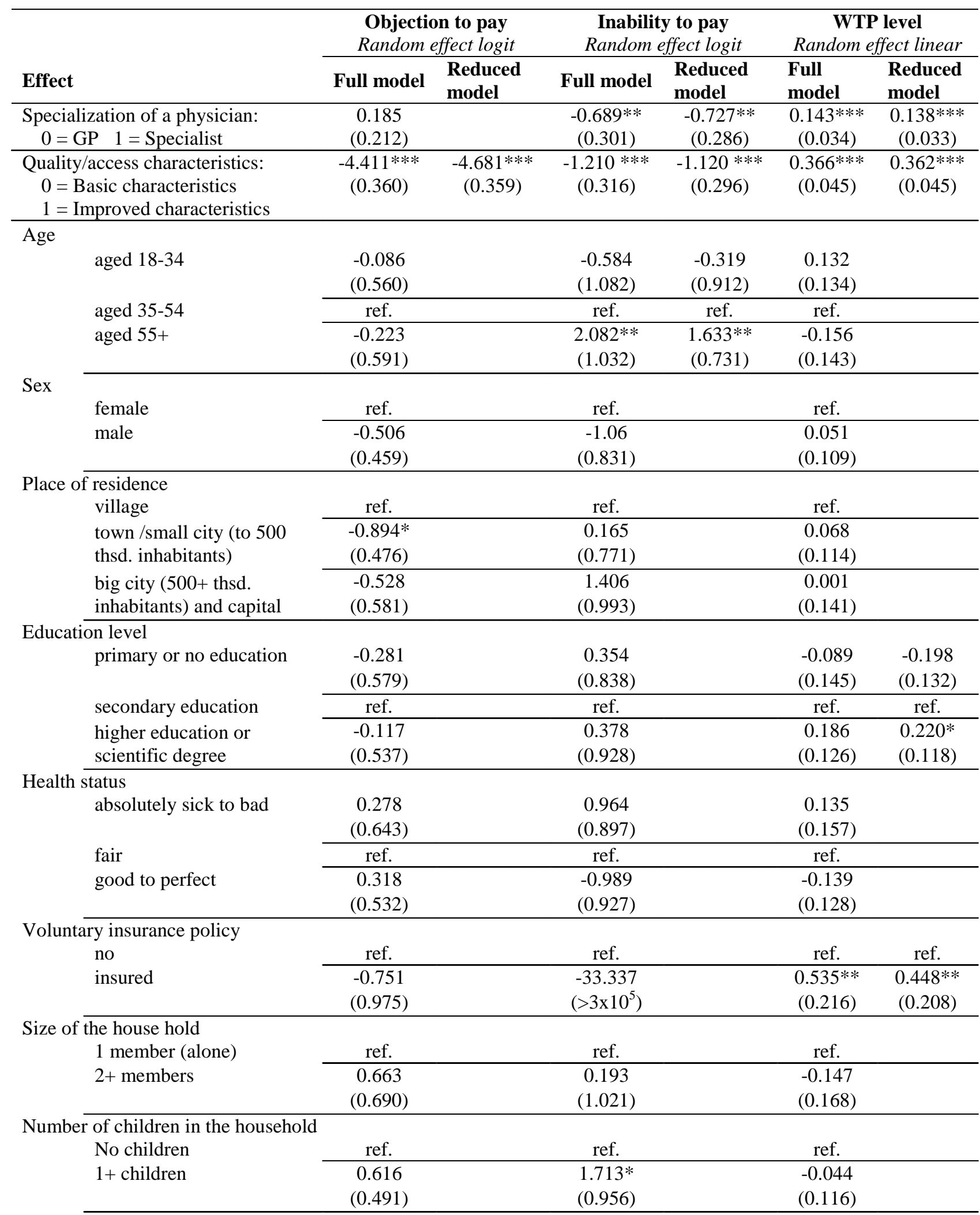

Share of household members who do not earn
half or less
more than half
ref. ref.
ref.
ref.
ref.

$\begin{array}{ccccc}-1.102^{* *} & -1.039^{* *} & 1.748^{* *} & 2.561^{* * *} & -0.016 \\ (0.488) & (0.425) & (0.800) & (0.685) & (0.115)\end{array}$

Significance: ${ }^{*} p<0.1 ;{ }^{* *} p<0.05 ;{ }^{* * *} p<0.01 ; \quad$ ref. - reference group; 
Table 3.3 (continued) Results of modeling WTP: objection to pay, inability to pay, and level of positive WTP

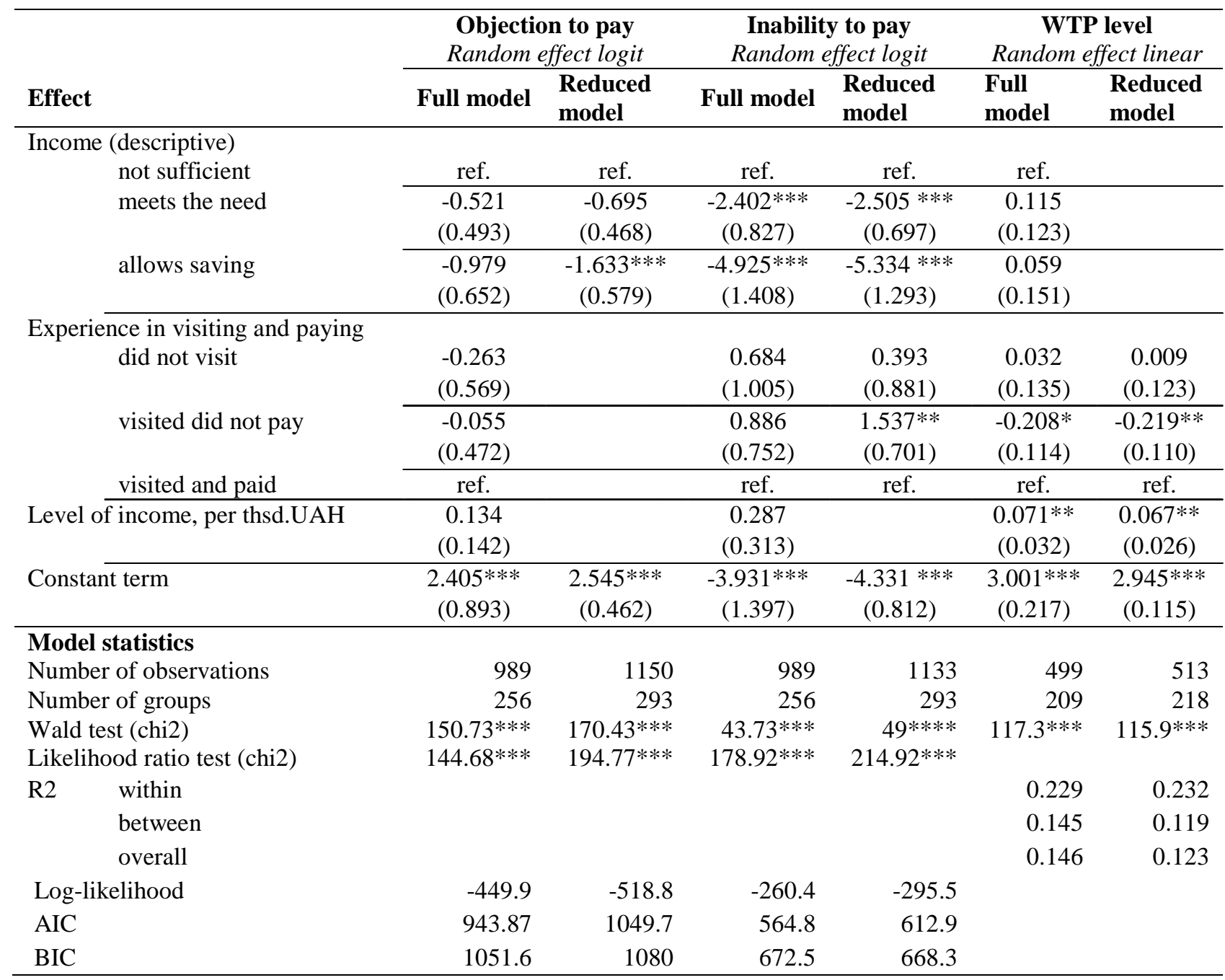

Significance: $* p<0.1 ; * * p<0.05 ; * * * p<0.01 ; \quad$ ref. - reference group

The indicators of economic status also significantly impact the probability of objecting to pay for physician services. Respondents living in households where most members (or respondents themselves) do not earn an income are less likely to object to pay. On the other hand, a high perceived household income (one that allows savings) might also reduce the inclination to object, though this effect is significant only in the reduced version of the model. An unstable effect, which disappears when the model is reduced to significant factors only, is found for the place of residence with town or small city residents being less likely to object. Other socio-demographic factors demonstrate no effect on the likelihood of objecting to pay. 
Inability to pay is related not only to the profile characteristics, but also to the specialization of a physician. The effect of the quality/access improvements is smaller compared to the model of objection to pay, but still highly significant, with more attractive profiles having 3 3.3 times lower odds to yield an inability-to-pay response. A similar effect is observed for the specialization of the physician but with a slightly lower magnitude: a medical specialist has a twice lower chance of yielding zero WTP due to inability to pay.

Akin to objection to pay, inability to pay is significantly reduced by the increase in the share of household members who earn an income and by the perceived income. However, unlike the objection to pay, respondents from households with more nonearning members are more likely to report that they are unable to pay. Perceived income is also a very strong predictor of inability to pay irrespective of the model specification, with higher income levels being associated with lower chances to report inability to pay. It is also notable that people aged 55 or more are much more likely to report being unable to pay (odds are 5 to 8 times higher than for the middle-aged). Also, people who visited a physician during the last 6 months but did not pay for this are more likely to report being unable to pay, though this effect is not stable.

The level of positive (nonzero) WTP, akin to ability to pay, is strongly and significantly related to both profile characteristics and specialization of the physician. Respondents report a higher WTP for a medical specialist (around 14\% more than for a GP) and for more attractive quality/access profiles (around 36\% higher). A strong and stable significant positive effect on WTP is observed for respondents with voluntary health insurance which is associated with a 45-53\% higher WTP. The WTP level is related to the monetary income level but not to the perceived one. Each $1000 \mathrm{UAH}$ (approximately $€ 87$ ) increase in household income is associated with around 7\% increase in the WTP level. Finally, experience in paying and visiting a physician has a significant and rather stable effect similar to the one for inability to pay. Respondents who visited a physician during the last 6 months and did not pay for this report a 20-22\% lower WTP than those who paid.

\subsection{Discussion and conclusion}

In this chapter, we have analyzed the potential and feasibility of official patient charges for public health care services in Ukraine. We addressed this issue by studying the patterns of fee acceptability, ability and WTP for physician services across population groups. The interpretation of the results should be done with some caution due to the limitations of the 
study. The results might be affected to some extent by the small sample size. Therefore, we focus our conclusions on strong main relations that are not sensitive to the model specification. Estimations based on bigger samples might show more detailed variations of acceptability, ability and WTP for physician services across population groups.

Another limitation relates to the methodology. CV is known to be subject to hypothetical bias (Loomis, 2011). That is respondents might not behave in the real world in the same way as they stated in a hypothetical experiment. However, some empirical studies have shown that open-ended CV (such as that used in our study) produces effect sizes that are rather comparable to real world WTP values (e.g. Ryan and Watson, 2009). Nevertheless, we are not inclined to interpret the mean WTP as an indication of the possible service fee because this requires detailed analysis of demand behavior under different payment regimes. Our results should be interpreted in terms of the mere existence of the potential for patient co-payments and the main value drivers for the patients. They may also serve as an indication of the societal benefits obtained through consumption of services of a given quality.

In the CV task respondents were presented with the scenario of an official fee. Therefore, their WTP statements might be affected by their attitudes towards formal and informal payment practices. Not all Ukrainians are positive about paying formally (Gryga et al., 2010). Formal charges are not part of the personal communication between patient and physician. Thus, they do not necessarily add to the coverage of personnel cost (i.e. physician's income) and do not assure better quality (i.e. quality and access desired by the patient). Besides, they may be charged on top of the informal charges causing a double burden for the patient. Moreover, Ukrainians are well aware of the fact that the official salary rate in the health care sector (1 555 UAH in December 2009 or around $€ 135$ ) is one of the lowest compared to other sectors of the Ukrainian economy (State Statistical Service of Ukraine, 2010a). Patients in many cases may perceive informal payments as an act of solidarity and a necessary supplement to the miserable official salary of physicians (Stepurko et al., 2012). Taking these perceptions into account we might expect that on average the true WTP level of the respondents is higher than the ones stated in the presented CV task due to lack of trust in official financing channels.

Our results demonstrate that official patient charges have potential in Ukraine. Even when faced with less attractive characteristics, Ukrainians express a rather substantial level of WTP, although less than a quarter of them are willing to pay. However, for physician services with improved quality/access characteristics, the share of those willing to pay is more than $70 \%$ 
with an average WTP of 44.8 UAH for a visit to GP (€ 3.9) and 51.9 UAH for a medical specialist (€ 4.5). There are no reliable estimates of the cost of health care services in Ukraine due to the existence of the public funding system where facilities are financed on a line-item budget principle regardless of the number of services provided. These stated WTP levels, however, are rather substantial in comparison to the average monthly salary in the health care sector. Taking into account that primary care specialists are among the low-income medical workers, the stated WTP on average appear comparable or even higher than the official personnel costs. Thus, co-payments in primary care in Ukraine can be regarded as a policy tool for raising additional income, especially in light of the necessary quality improvements.

The introduction of co-payments in the public health sector may have various effects both in terms of consumption patterns and the official cost of the services. The effects on consumption should be subject of demand modeling studies. From the system and provider's perspective, co-payments generate additional funds that could be redistributed to achieve different goals. Our results suggest that Ukrainians place high economic value on quality and access improvements. Thus, patient charges can only be implemented together with effective investment policies targeted at improving quality and access. The probability to object to pay for these services is mostly explained by low quality/access characteristics. Additionally, the likelihood of the ability to pay and the level of positive WTP are positively and strongly related to the quality/ access profile.

Combined with the evidence that Ukrainians in general are not satisfied with the quality of care they receive (Gorshenin Institute, 2011) these findings underline the necessity of quality/access improvements in health care. A rough and conservative estimation (the difference of the mean WTP in Table 3.2, objection answers excluded) suggests that the social benefit gained from simple improvements in the state of medical equipment, maintenance of the physician office, and reduction of waiting time is $16.5 \mathrm{UAH}$ per visit to a GP and 23.0 UAH per visit to a medical specialist (in December 2009 prices). In light of the planned health care reforms, this can be regarded as an indication of the investment potential, although more robust estimates based on larger samples may provide more precise indicators. In Ukraine, increasing quality and access can not only be realized through investments in training, capital, and organizational changes, but is also tightly related to personnel remuneration. Failure to satisfy physicians' needs may provoke both resistance to official charges and double charges: formal on top of informal. 
Our results demonstrate that among the zero WTP answers, protesters (the objection motive) are not driven by economic or social barriers. The negative relation with the share of nonworking members in the household only supports this idea: it indicates that the more members depend on one's alimony and, the more responsibility one has for else's health and life. This might increase the value of health care service and, consequently, decrease the likelihood of objection to pay despite the (in)ability to pay. Moreover, reporting objection to pay does not necessarily lead to similar behavior in real life as it is not related to the payment experience in the year before the survey (i.e. chances of paying in real life are similar for those who object to pay and for those who do not object). Thus, both from a methodological and a policy perspective it is rational not to account for the preferences of pure 'protesters'.

As for the ability/inability to pay and the level of WTP, objective socio-economic barriers, such as age and economic status, apply. The relations with household income also support the theoretical validity of the construction of the models. It is notable however that inability to pay is related to the perceived income level, while the level of WTP is related to monetary income. This shows that inability is an issue of perception depending on the evaluation of one's own income level, while the level of WTP is defined by real monetary budgetary constraints. This suggests that different mechanisms underlie the two stages of the decision about WTP for the physician services and this should be accounted for in the WTP modeling.

The substantial share of the population that is unable to pay for physician services (at least $11.5 \%$ for the medical specialist with attractive characteristics) is concerning. This is in line with the extensive discussions in the literature (e.g. Tambor et al., 2011; Sepehri and Chernomas, 2001) that patient charges should be implemented together with exemption criteria related to age and income. To relate co-payment levels to the level of income might also help to reduce financial barriers to access although this is difficult to achieve in practice. A successful example is Bulgaria where patient charges are anchored on the minimum income in the country (Atanasova et al., 2011) although this does not eliminate barriers to access.

It is also worth mentioning that we observe a slight preference among Ukrainians for a direct referral to medical specialist and bypassing a GP. This is expressed through the higher likelihood of reporting inability to pay and the lower WTP level for the latter. However, this preference is practically non-existent for services with less favorable characteristics although a bit more explicated for services with more attractive quality/access characteristics. In the latter case, people are willing to sacrifice only around 7 - $8 \mathrm{UAH}$ to bypass primary contact. This indicates that price signals (such as penalizing fees for direct referral to a specialist) 
might still be necessary to discourage bypass practices if official patient charges are introduced. However, the variation of this preference across population groups should be studied in more detail in order to design an effective threshold for discouraging direct visits to a specialist without a referral.

To our knowledge WTP for physician services in Ukraine has never been studied before. Thus, testing the external validity using other studies may only be done in relative terms. In our study, WTP estimates range from $0.9 \%$ to $1.9 \%$ of household income when protest answers are excluded and from $1.6 \%$ to $2.2 \%$ when only positive values are considered. This is rather consistent for example with the results from Spain where WTP for physician outpatient services represent 2\% of household income (Martín-Fernández et al., 2010b).

Thus, the results of this chapter have implications for both the methodology of CV and for patient payment policies. From the methodological perspective, our findings suggest that that acceptability/objection to pay is mostly related to quality/access characteristics of the services and is not to socio-economic characteristics. Hence, the protesters do not seem to attach an intrinsic 'zero value' to the services, and should be excluded from the estimations of WTP. At the same time, the inability to pay and the level of WTP are related to socio-economic factors. Our results demonstrate that the potential of patient charges for physician services is promising as the level of WTP for physician services is substantial despite the quality/access profile of the services. However, if patient charges are implemented, the lower ability and WTP among vulnerable groups should be addressed by well-designed exemption criteria based on age and income and by anchoring co-payment levels on income.

Importantly, patient charges cannot be implemented without quality/access improvements in Ukraine. The social benefits that can be gained from quality improvements in medical equipment, maintenance and a reduction in waiting time (expressed through an increase in mean WTP) are rather substantial. Additionally, we find a rather weak (around 7 UAH per visit) monetary preference for direct referral. Thus, in the context of strengthening the role of primary care, differential patient charges for different service levels may be called for. 



\section{CHAPTER 4}

Willingness to pay for physician services:

Comparing estimates from a discrete choice experiment and contingent valuation

Based upon:

Danyliv, A., Pavlova, M., Gryga, I., \& Groot, W. (2012). Willingness to pay for physician services: Comparing estimates from a discrete choice experiment and contingent valuation. Society and Economy, 34(2), 339-357. DOI: 10.1556/SocEc.34.2012.2.9 


\section{Chapter 4}

\section{Abstract}

Background: DCE and CV are often applied to value health care benefits. However, whether the two techniques yield converging WTP estimates is not studied well.

Objective: This study aims to compare at a disaggregated level WTP estimates for physician services obtained from DCE and CV estimates. We study the consistency between the estimates and whether there are systematic differences between the two.

Methods: The analysis was based on data from a small-scale household survey in Ukraine, the same as in Chapter 2 and 3 of the dissertation. The sample included 303 respondents and was taken to be representative of the Ukrainian population. The respondents participated in both DCE (16 choice tasks) and CV (4 valuation scenarios) in a form of payment scale followed by open-ended questions about the exact maximum WTP.

Results: We find that DCE produces higher WTP estimates than CV does, and the estimates are not consistent across the two techniques. A difference between the WTP estimates from DCE over those derived from the CV technique is found (i) for respondents who do not discriminate well between the profiles, and (ii) for an increase in the presented attribute level changes.

Conclusions: A better convergence between the WTP estimates from two techniques may be achieved if the measures are taken to increase the relevance of the valuation tasks to the respondents, but also if certain design and empirical strategies are applied. 


\subsection{Introduction}

In estimating the economic value of health care goods and services or their attributes, two SP techniques, namely CV and DCE, play an increasingly important role. In Chapter 1, section 1.2, recent applications of those methods in health care were reviewed (for an overview see also Hanley et al., 2003; Ryan et al., 2001b). Empirical studies comparing both techniques using the same data are scarce (Bijlenga et al., 2011; Boxall et al., 1996; Hanley et al., 1998; Ryan, 2004a; Ryan and Watson, 2009; Taylor and Armour 2002; van der Pol et al., 2008). These comparative studies mostly agree that WTP estimates from DCE technique are higher than those from open-ended CV formats, and close to those from close-ended CV formats. However, most of these studies compare aggregate WTP estimates. It is therefore unclear whether the WTP estimates from the two techniques differ systematically and what factors contribute to the discrepancy (Boxall et al., 1996; Hanley et al., 1998; Ryan, 2004a; Ryan and Watson, 2009; Taylor and Armour, 2002; van der Pol et al., 2008). Moreover, these aggregate comparisons show a different extent of discrepancy.

The question of a systematic difference or even convergence of the WTP estimates from CV and DCE methods is important because if convergence can be achieved the choice of the appropriate technique would be only in convenience and design. In contrast, the absence of a systematic difference or even divergence of these techniques raises the question of which one is more valid and should be used in decision-making. As far as there is no agreement in the literature on the superiority of one of the techniques, there is still much to be done in investigating and improving their convergence. There is evidence in the literature that the DCE design and model issues such as 'opt-out' option (Ryan and Skåtun, 2004), non-linear function (Hanson et al., 2005), and price levels (Slothuus Skjoldborg and Gyrd-Hansen, 2003) affect the difference between aggregate WTP estimates from DCE and CV techniques. The discrepancy may also arise from the respondents' behavior. This has not been addressed empirically yet.

In this chapter, we aim to study empirically which factors at the disaggregate level contribute to the difference in WTP estimates for physician services obtained from DCE and CV techniques. Based on this we discuss what design aspects may improve consistency between the estimates from two techniques. The analysis is based on data from 303 respondents representing the Ukrainian population. All respondents participated in both DCE (16 choice tasks) and CV (4 valuation scenarios). 


\subsection{Background}

There are few empirical comparisons of WTP estimates from DCE and CV. Seven such studies were identified by a literature search (Bijlenga et al., 2011; Boxall et al., 1996; Hanley et al., 1998; Ryan, 2004a; Ryan and Watson, 2009; Taylor and Armour, 2002; van der Pol et al., 2008). We do not consider comparisons within the CV technique, e.g. between the discrete choice format (referendum question) and other formats of direct statements of which there are many. Also, we do not consider comparisons within the DCE technique. Five of the studies belong to the health economics field while the others come from environmental economics.

Generally, two main aspects of convergence between the WTP estimates from DCE and CV techniques are considered in these publications: (i) systematic excess, i.e. the WTP values from one technique are systematically higher than those from the other technique, at least at the range under study; and (ii) consistency, i.e. those who state higher WTP in CV are assigned higher WTP values based on DCE modeling, and vice-versa.

As for the first aspect of convergence, systematic excess, the literature suggests that WTP estimates derived from DCE would be close to those from CV in discrete choice or closeended (referendum) format (Hanley et al., 1998; Ryan, 2004a), but would be overall substantially and significantly higher than those from CV in open-ended or payment cards format (Bijlenga et al., 2011; Ryan and Watson, 2009; van der Pol et al., 2008). Only one study from environmental economics (Boxall et al., 1996) reports higher WTP estimates from CV in discrete choice format, compared to WTP from DCE, but the magnitude of the discrepancy was biased by the design of choice tasks.

The potential causes for the excess in DCE estimates of WTP are not well studied. It appears that in DCE linear specification of the utility function (Hanley et al., 1998) and absence of substitutes (Boxall et al., 1996) (e.g. 'opt-out' option) may increase and, consequently, enhance overestimation of the actual WTP mentioned above. On the other hand, the excess could be also linked to the levels of the price attribute presented in DCE, though there is a controversy in the literature if this effect takes place. For example, Ryan and Wordsworth (2000) find no evidence that the levels of the price attribute have an impact on the magnitude of WTP in DCE, while the study by Slothuus Skjoldborg and Gyrd-Hansen (2003) proves the opposite. In any case, the potential effect of price levels should be accounted for in comparative studies such as ours. 
The second aspect of convergence between the WTP estimates from DCE and CV techniques, consistency, has been studied in few papers so far (Bijlenga et al., 2011; van der Pol et al., 2008). This can be partially explained by the fact that it requires disaggregate comparisons, where both WTP estimates are available for each respondent. We identified two studies that apply open-ended format to CV and compare it to DCE (Bijlenga et al., 2011; van der Pol et al., 2008). Both studies find a high level of consistency between WTPs from DCE and CV. Van der Pol et al. (2008) derive this conclusion based on the observation that residuals from the CV model are positively interacted with the price attribute in the DCE model, while Bijlenga et al. (2011) simply find a strong positive linear relationship between the predicted WTP from DCE and the stated WTP from CV, and good concordance between coefficients in CV and DCE models. It should be noted that while the latter study applies a heavy CV design maximally matching the DCE questions, the former is based on one CV task only revealing WTP for an 'ideal package'. Nevertheless, these findings would make us expect that there is an adequate consistency between the WTP estimates from the two techniques - CV and DCE.

Another aspect of convergence between the WTP estimates from DCE and CV techniques, which has been studied only sporadically yet, is related to the determinants of systematic differences between these WTP estimates, i.e. which factors define the discrepancy between the estimates. However, we consider this to be a key aspect because knowledge of the sources of the discrepancies may indicate the direction of potential improvements in the validity of both methods. From this, we may assume that a more realistic framing such as the introduction of a non-linear utility function, introduction of the 'opt-out' option, and meaningful price levels (and other attributes) may bring WTP estimates from DCE closer to WTP estimates from open-ended CV formats, and possibly, to the actual WTP levels (Lancsar and Louvierre, 2008; Lancsar et al., 2007). However, discrepancy can also emerge from strategic behavior of respondents rather than from the technical design of the tasks. This source of discrepancy can be captured by socio-demographic characteristics of the respondents to check if discrepancy occurs in a specific socio-demographic group, but it could also be independent of socio-demographic features e.g. due to a specific perception of the presented tasks. In this chapter, we offer a way to approach the latter issue.

As discussed in Chapter 1, closed-ended choice-based formats of the CV study are seen as theoretically valid (e.g. Carson, 2000). In contrast, empirical studies, as was already discussed, reveal that the open-ended format of CV provides WTP estimates that better reproduce the actual maximum WTP in a real market (e.g. Frew et al., 2003; Ryan and 


\section{Chapter 4}

Watson, 2009). Close-ended (binary choice) and bidding format WTP estimates are higher (Frew et al., 2003; Whynes et al., 2005) but closer to those from DCE. Such patterns are explained by different incentives for preference revelation in open- and close-ended formats, while in choice-based CV and DCE, these incentives are similar. Thus, it can be assumed that choice-based CV and DCE provide inflated results compared to actual choices. Therefore, in this chapter we are particularly concerned with what defines the difference in WTP estimates from techniques with incompatible incentives: open-ended CV and DCE.

\subsection{Methods}

In previous chapters, i.e. Chapter 2 and 3 DCE and CV data from a small-scale household survey were analyzed. In this chapter we exploited the same data from 303 respondents representing adult Ukrainian population. Details about sampling strategy are presented in the Appendix A. Sixteen DCE tasks preceded four CV questions in the questionnaire. For both techniques, physician profiles were created based on six quality and access attributes, described in Chapter 2 in detail. Specialization of the physician and state of medical equipment represented some aspects of perceived clinical quality which patients may know or have some expectations about before making the real choice. Social quality was represented by maintenance of the physician office and attitude of the medical staff. Finally travel and waiting time were the two aspects of access (or cost of time). The DCE task additionally included two attributes of cost: size and type of payment (formal or informal), while the CV task required direct statement of WTP for a visit to a physician represented through other six attributes.

The DCE design was described in Chapter 2 in detail. In brief, it implied that each respondent should pass 16 choice tasks. The example of the DCE task may be found in Appendix C. The question of a maximum plausible number of choice tasks is disputable. As discussed in Chapter 1, some recent studies apply as much as 32 choice tasks (e.g. Bijlenga et al., 2011), while others argue that lower numbers, i.e. 12 or 16 are only manageable (e.g. Van der Pol et al., 2008; Ryan et al., 2001). Due to the fact that the price attribute has 3 levels while all the other attributes have only 2, the level balance was not fully reached.

CV contained four valuation tasks asking for a direct statement of WTP for four physician profiles: a general practitioner with some basic characteristics (basic GP profile), a general practitioner with the best possible characteristics (best GP profile), a medical specialist with 
some basic characteristics (basic specialist profile), and a medical specialist with the best possible characteristics (best specialist profile) (see Table 4.1). The detailed description of the CV design may be found in Chapter 3 and the example of the task - in Appendix C.

We keep to the view that results of DCE modeling in our study allows estimating WTP for joint or separate changes in physician's attribute. Therefore, in order to become comparable to WTP estimates from DCE, we transformed the direct WTP statements from the CV task for the four profiles into three differences in stated WTP (henceforth designated as $W T P_{C V}^{\Delta}$ ), For this purpose, the basic GP profile was chosen as a basis and WTP for this profile stated by a respondent was compared to WTP stated by the same respondents for: (1) the best GP profile, (2) the basic specialist profile, (3) the best specialist profile. The transformations described above are presented in Table 4.1. Such transformations allowed estimating separately two effects. First, the effect of the change in the specialization of a physician could be estimated, and second, the effect of the joint change in three quality factors: from outdated to modern equipment, from bad to good maintenance of the physician's office, and from long (45 minutes) to short (10 minutes) waiting time. Two other factors - attitude of the medical staff and travel time, did not vary in the CV task.

Table 4.1 Attributes of physician services for CV and the attribute differences for changes in profile

\begin{tabular}{|c|c|c|c|c|c|c|c|c|}
\hline \multirow{2}{*}{ Attribute } & \multirow{2}{*}{$\begin{array}{l}\text { Possible levels of } \\
\text { attributes (from } \\
\text { DCE) }\end{array}$} & \multicolumn{4}{|c|}{ CV profiles } & \multicolumn{3}{|c|}{$\begin{array}{c}\text { Differences for } \\
\text { empirical modeling }\end{array}$} \\
\hline & & $\begin{array}{c}\text { GP } \\
\text { basic }\end{array}$ & $\begin{array}{l}\text { GP } \\
\text { best }\end{array}$ & $\begin{array}{c}\text { MS } \\
\text { basic }\end{array}$ & $\begin{array}{l}\text { MS } \\
\text { best }\end{array}$ & $\begin{array}{l}\text { GP0- } \\
\text { GP1 }\end{array}$ & $\begin{array}{l}\text { GP0- } \\
\text { MS0 }\end{array}$ & $\begin{array}{l}\text { GP0- } \\
\text { MS1 }\end{array}$ \\
\hline $\begin{array}{l}\text { Physician's } \\
\text { specialization }\end{array}$ & $\begin{array}{l}1=\text { Medical specialist } \\
0=\text { General } \\
\text { practitioner }\end{array}$ & 0 & 0 & 1 & 1 & 0 & +1 & +1 \\
\hline $\begin{array}{l}\text { State of the medical } \\
\text { equipment }\end{array}$ & $\begin{array}{l}1=\text { Modern } \\
0=\text { Outdated }\end{array}$ & 0 & 1 & 0 & 1 & +1 & 0 & +1 \\
\hline $\begin{array}{l}\text { Maintenance of the } \\
\text { physician's office }\end{array}$ & $\begin{array}{l}1=\text { Renovated } \\
0=\text { Old-looking }\end{array}$ & 0 & 1 & 0 & 1 & +1 & 0 & +1 \\
\hline $\begin{array}{l}\text { Attitude of the } \\
\text { medical staff }\end{array}$ & $\begin{array}{l}1=\text { Polite } \\
0=\text { Arrogant }\end{array}$ & 1 & 1 & 1 & 1 & 0 & 0 & 0 \\
\hline $\begin{array}{l}\text { Travel time to the } \\
\text { physician's office }\end{array}$ & $\begin{array}{l}60 \min \\
15 \min \end{array}$ & 15 & 15 & 15 & 15 & 0 & 0 & 0 \\
\hline $\begin{array}{l}\text { Waiting time in front } \\
\text { of the physician's } \\
\text { office }\end{array}$ & $\begin{array}{l}45 \min \\
10 \mathrm{~min}\end{array}$ & 45 & 10 & 45 & 10 & -35 & 0 & -35 \\
\hline
\end{tabular}

The respondent's $W T P_{C V}^{\Delta}$ for changes in physician profiles are compared to the marginal WTP for the same profile changes predicted for that respondent based on the results of DCE 


\section{Chapter 4}

modeling (henceforth designated as $W T P_{D C E}^{\Delta}$ ). Results of the DCE modeling used for the estimation of $W T P_{D C E}^{\Delta}$, were presented above in the Chapter 2 (see Table 2.2). By using a random parameter logit specification, we address unobserved heterogeneity of tastes by including random coefficients. The observed taste heterogeneity was addressed by the inclusion of interactions between attributes and socio-demographic characteristics. Predictions were done for each observation based on the formula for marginal WTP which accounted for changes in service attributes and for socio-demographic characteristics of a respondent as well (Lancsar et al., 2007). Our analysis was carried out at a disaggregate level.

The question of the systematic excess of the WTP estimate from one technique over the other was approached by the comparison of stated $W T P_{C V}^{\Delta}$ and predicted $W T P_{D C E}^{\Delta}$ based on a paired t-test. In addition to this, consistency between them was assessed by applying Pearson's correlations and linear regression. A significantly positive relation indicates that techniques produce consistent WTP estimates. Eventually, determinants of systematic difference between $W T P_{C V}^{\Delta}$ and $W T P_{D C E}^{\Delta}$ estimates (designated as $\Delta W T P^{\Delta}=W T P_{D C E}^{\Delta}-W T P_{C V}^{\Delta}$ ) were analyzed by regressing this difference on three groups of factors, representing potential sources of discrepancy: socio-demographic features of respondents, the changes in the attribute levels appearing in the profile changes under valuation, and the difference in the stated $W T P_{C V}^{\Delta}$.

Socio-demographic characteristics indicated if there were certain population characteristics that affected the difference in the WTP measures. Level changes in the attributes were introduced to indicate if the discrepancy was related to the design of the profiles. Finally, the introduction of the difference $W T P_{C V}^{\Delta}$ was motivated by the assumption that irrelevant or zero value changes in the profiles, presented by zero or low values of this factor, should result in less consistent behavior of respondents in DCE tasks with no possibility to opt out.

\subsection{Results}

The comparison of WTP estimates from the two techniques (i.e. between individual $W T P_{C V}^{\Delta}$ and $W T P_{D C E}^{\Delta}$ for all three changes in profile: from basic GP to best GP, basic specialist and best specialist profile respectively) with paired t-test shows that DCE produces overall higher estimates than CV. The correlation between these two estimates is positive but low and rises dramatically when outliers (7\% of cases where $W T P_{D C E}^{\Delta}$ is higher than $200 \mathrm{UAH}$ ) are 
excluded. In particular, the correlation coefficient increases from 0.196 to 0.416 . However, if we estimate the correlation coefficient of each change in profile separately, this relation disappears as the three new correlation coefficients are very low (Pearson's correlation coefficients are between 0.024 and 0.076). Therefore, the evidence of consistency between the two estimates is rather weak. Hence, DCE in our case produces systematically higher WTP estimates than CV does; however, these estimates are not consistent between each other.

Graphically it was noticed that there is a relation - very close to linear - between the excess $\Delta W T P^{\Delta}$ and the difference $W T P_{C V}^{\Delta}$, which becomes rather strong when the three changes in the profiles are considered separately and outliers are excluded (Pearson's correlation coefficient ranges from $-0,489$ to $-0,784)$. The analysis of the determinants of the systematic differences (in this case excess) of $W T P_{D C E}^{\Delta}$ and $W T P_{C V}^{\Delta}$ estimates (i.e. $\triangle W T P^{\Delta}$ ) is summarized in Table 4.2. As can be seen in Table 4.2, the regression analysis included indicators for the profile change (change in specialization and quality improvements), sociodemographic characteristics, and interactions of $W T P_{C V}^{\Delta}$ with these characteristics. After testing the model for inclusion of different groups of factors, it is concluded that most of the variation in the excess of $W T P_{D C E}^{\Delta}$ over $W T P_{C V}^{\Delta}$ can be explained by the joint effect of the change in $W T P_{C V}^{\Delta}$, the change of the specialization of the physician and quality improvements in the profile (model 1: R2=0.721). Attribute changes only, such as change of the specialization of the physician and quality improvements in the profile, explain substantially less of $\Delta W T P^{\Delta}$ variation (model 2: R2=0.419). Further adding socio-demographics and interactions of $W T P_{C V}^{\Delta}$ with all the other factors, improves slightly the model fit (model 3: $\mathrm{R} 2=0.886)$.

From the results in Table 4.2, it can be concluded that $1 \mathrm{UAH}$ increase in the $W T P_{C V}^{\Delta}$ reduces the discrepancy in WTP estimates between the two techniques approximately by the same value (coefficient is around -1). A change in specialization from GP to medical specialist increases this discrepancy by around $10 \mathrm{UAH}$, while the presence of a joint improvement in the 3 quality attributes, namely medical equipment, maintenance of the office and reduced waiting time, increases this discrepancy by 105.85 - 107.67 UAH. 


\section{Chapter 4}

Table 4.2 Regression of the discrepancies of WTP estimates over quality changes and effects of socio-demographic characteristics.

\begin{tabular}{|c|c|c|c|c|}
\hline & Model 1 & Model 2 & Model 3 & Model 4 \\
\hline Effect & $\begin{array}{l}\text { Only } 3 \\
\text { factors }\end{array}$ & $\begin{array}{l}\text { Only } \\
\text { WTP }^{\mathrm{D}} \mathrm{cv}\end{array}$ & $\begin{array}{c}\text { Only } \\
\text { attribute } \\
\text { changes }\end{array}$ & $\begin{array}{l}\text { Model full- } \\
\text { reduced }\end{array}$ \\
\hline $\mathrm{WTP}^{\mathrm{D}} \mathrm{CV}$ & $\begin{array}{r}-0.993 * * * \\
(0.043)\end{array}$ & $\begin{array}{r}-0.282 * * * \\
(0.071)\end{array}$ & & $\begin{array}{r}-1.010 * * * \\
(0.084)\end{array}$ \\
\hline $\begin{array}{l}\text { Attribute changes: } \\
\text { dx1 (GP to MS) }\end{array}$ & $\begin{array}{r}10.311 * * * \\
(3.083)\end{array}$ & & - & $\begin{array}{r}10.359 * * * \\
(2.061)\end{array}$ \\
\hline dx2 (3 attributes improvement) & $\begin{array}{r}105.858 * * * \\
(3.351)\end{array}$ & & $\begin{array}{r}69.475 * * * \\
(3.704)\end{array}$ & $\begin{array}{r}107.670 * * * \\
(2.310)\end{array}$ \\
\hline Constant term & $\begin{array}{l}-0.797 \\
(3.720)\end{array}$ & $\begin{array}{r}61.526 * * * \\
(2.628)\end{array}$ & $\begin{array}{r}11.670 * * * \\
(2.973)\end{array}$ & $\begin{array}{c}0.096^{* *} \\
(4.420)\end{array}$ \\
\hline
\end{tabular}

\section{Socio-demographic characteristics}

age (younger than 20)

sex (male)

education (higher or doctoral)

activity (not working)

activity (retired)

health (very good or perfect)

health (poor and very poor)

disabled

private insurance

1-2 children

higher income (>1000UAH)

residing in rural area

residing in small city

residing in middle city

residing in capital (Kyiv)

$30-80 \%$ of household not working

$80-100 \%$ of household not working 
Table 4.2 (continued) Regression of the discrepancies of WTP estimates over quality changes and effects of socio-demographic characteristics.

\begin{tabular}{|c|c|c|c|c|}
\hline & Model 1 & Model 2 & Model 3 & Model 4 \\
\hline Effect & $\begin{array}{l}\text { Only } 3 \\
\text { factors }\end{array}$ & $\begin{array}{c}\text { Only } \\
\mathrm{WTP}^{\mathrm{D}} \mathrm{cV}\end{array}$ & $\begin{array}{c}\text { Only } \\
\text { attribute } \\
\text { changes }\end{array}$ & $\begin{array}{l}\text { Model full- } \\
\text { reduced }\end{array}$ \\
\hline \multicolumn{5}{|l|}{ Interactions } \\
\hline \multicolumn{5}{|l|}{$\mathrm{WTP}^{\mathrm{D}} \mathrm{cv}$ by } \\
\hline activity (retired) & & & & $\begin{array}{r}-0.263^{* *} \\
(0.125)\end{array}$ \\
\hline missed more than 14 days due to ill. & & & & $\begin{array}{r}-0.349 * * * \\
(0.093)\end{array}$ \\
\hline residing in capital (Kyiv) & & & & $\begin{array}{r}-0.481 * * * \\
(0.091)\end{array}$ \\
\hline very good or perfect & & & & $\begin{array}{r}-0.419 * * * \\
(0.111)\end{array}$ \\
\hline activity (not working) & & & & $\begin{array}{r}-0.335^{* * * *} \\
(0.080)\end{array}$ \\
\hline $80-100 \%$ of household not working & & & & $\begin{array}{r}0.512 * * * \\
(0.115)\end{array}$ \\
\hline no missing days due to illness & & & & $\begin{array}{r}0.152 * * \\
(0.069)\end{array}$ \\
\hline residing in small city & & & & $\begin{array}{r}0.378 * * * \\
(0.107)\end{array}$ \\
\hline residing in middle city & & & & $\begin{array}{r}0.188 * * * \\
(0.072)\end{array}$ \\
\hline residing in rural area & & & & $\begin{array}{r}0.800 * * * \\
(0.260)\end{array}$ \\
\hline having private insurance & & & & $\begin{array}{r}-0.284^{* *} \\
(0.121)\end{array}$ \\
\hline male & & & & $\begin{array}{r}-0.131^{* *} \\
(0.066)\end{array}$ \\
\hline no or primary education & & & & $\begin{array}{r}-0.600 * * * \\
(0.120)\end{array}$ \\
\hline higher or doctoral education & & & & $\begin{array}{r}0.279 * * * \\
(0.064)\end{array}$ \\
\hline poor or very poor health & & & & $\begin{array}{r}-0.537 * * * \\
(0.140)\end{array}$ \\
\hline \multicolumn{5}{|l|}{ Model statistics } \\
\hline Number of observations & 489 & 489 & 489 & 489 \\
\hline F test & $417.04 * * *$ & $15.69 * * *$ & $351.82 * * *$ & $100.36 * * *$ \\
\hline R-squared & 0.7206 & 0.0312 & 0.4194 & 0.8858 \\
\hline Adj R-squared & 0.7189 & 0.0292 & 0.4182 & 0.8769 \\
\hline
\end{tabular}

Since the additional socio-demographic characteristics do not contribute considerably to the explanatory power of the model, we do not consider these factors in detail. Moreover, a small sample size may have caused many sporadic effects in small socio-demographic groups. Although the introduction of socio-demographic effects and interactions improves the model 


\section{Chapter 4}

fit, it does not change much the magnitude of the coefficients, and does not impact the direction or significance of the other three factors, which suggests that our results are not specific to a certain group.

\subsection{Discussion and conclusion}

The question of the comparison of WTP estimates derived from DCE and CV has been empirically studied insufficiently so far. It should be noted that there is no single view on whether WTP estimates from DCE and CV are comparable, neither there is a single vision of the correct methods of deriving and interpreting them (e.g. Lancsar and Savage, 2004). However, this study implies a technical comparison despite the nature of these estimates.

Our observation that WTP estimated from DCE is higher than the one produced by CV in payment card format is in concordance with most comparative studies (Bijlenga et al., 2011; Ryan and Watson, 2009; van der Pol et al., 2008). As was discussed in the background section, the overall excess in $W T P_{D C E}^{\Delta}$ estimates over the stated $W T P_{C V}^{\Delta}$ values can to some extent be explained by the absence of the opt-out option in the DCE tasks and linear utility specification. Therefore, the impact of these design aspects on the convergence of WTP values from the two techniques should be further considered.

The evidence for consistency between WTP estimates from CV and DCE techniques in our study is rather weak. This is in discordance with the other studies that found very high levels of consistency (Bijlenga et al., 2011; van der Pol et al., 2008). Bijlenga et al. (2011) used a CV design very similar to DCE tasks with 32 evaluated profiles, while in our study CV tasks were kept to minimum to estimate two separate effects. Van der Pol et al. (2008), in contrast to our study, used non-linear utility specification. Thus, it appears that non-linearity may also impact the consistency between WTP estimates.

Our observation that the discrepancy between predicted $W T P_{D C E}^{\Delta}$ and stated $W T P_{C V}^{\Delta}$ for the same change reduces with the increase of stated $W T P_{C V}^{\Delta}$ may be attributed to certain pattern in respondents' behavior. This implies that people stating low or zero additional WTP for improved profiles do not see added value in the changes in the presented attributes and, thus, they might either be less confident of their choices or pay less attention to the tasks and, consequently, behave less consistently in DCE tasks. This implies that such respondents might not discriminate between the profiles presented in the DCE task when attributes that are 
not relevant to them are varied. Their choices, if they are forced to choose, will be then erratic resulting in inconsistent welfare estimates.

From this perspective in the analysis of data from DCE it might be important to account only for those choice tasks from a respondent in which one can really discriminate between the presented profiles in terms of gained utility. Basically two strategies might be applied to do this: either excluding cases where a respondent does not see the relevance of the presented attribute changes, or apply an adaptive choice design. The former strategy would imply the introduction of an additional answer option allowing a respondent to state that the attributes varying in the task are not relevant to him/her. However, this strategy is problematic because, firstly, such answer option might confuse the respondent, and secondly, it might cause many drop offs and, hence, high cost of data. The latter strategy, i.e. the adaptive choice design (e.g. Johnson et al., 2003), allows incorporating the answers of all respondents into analysis as it defines as a first step which attributes are most relevant to the respondent and then builds further choice-tasks based on relevant attributes only. This approach is also subjected to difficulties with administration as it requires special software and computer dealt questionnaires, which may be impossible in many contexts. These two strategies should be considered as means of reducing the noise in data coming from respondents who are confronted with tasks that are irrelevant to them.

The discrepancy in WTP estimates from the two techniques can to a large extent be explained by the variation in profiles: mostly by quality improvements and less by a change in the specialization of a physician. This means that DCE tends to overestimate WTP more for the higher variations in attributes and less or not at all at lower levels. From a theoretical perspective such divergence might have arisen because of the linear specification of the utility function in the DCE model. Introduction of a convex utility function for price and time attributes and/or concave for other attributes (see Wassenaar et al., 2005) are expected to result in smaller effects for the higher values of positive attributes and larger effects for the expenditure (negative) attributes, which is more in line with consumer behavior theory (Lancsar et al., 2007). This should result in lower $\mathrm{WTP}_{\mathrm{DCE}}$ estimates at higher levels of attribute changes (that will affect the utility level less) leaving them practically unaffected at lower levels of attribute changes. Thus, $W T P_{D C E}^{\Delta}$ estimates might become closer in pattern to stated $W_{T P}^{\Delta}$. However, non-linear utility functions are not conventional in the DCE analysis and result in computationally slightly more complex solutions for $W T P_{D C E}^{\Delta}$ estimates. Further 


\section{Chapter 4}

research on the impact of non-linear utility functions on the convergence of the WTP estimates from two valuation techniques is necessary.

Another possible implication of the effect of attribute levels on the discrepancy between WTPs is that marginal changes rather than total variation changes in the levels of attributes for the DCE tasks are more relevant for consumer choice. Hence, they should produce $W T P_{D C E}^{\Delta}$ values closer to the difference in the directly stated $W T P_{C V}^{\Delta}$. However, it is not always possible to express other than extreme levels of the non-quantifiable attributes to the respondent (i.e. attitude can be polite or impolite, but not polite to some extent). Additionally, it might be difficult to find an average profile as in the actual market to be used as a reference and for constructing marginal changes, especially if the health care markets are uneven in terms of quality, access and price.

Finally, we do not find strong evidence for the impact of socio-demographic factors on the discrepancy between WTP estimates from DCE and CV. Some of them might affect it, but our results demonstrate that they explain not much of the discrepancy in the WTP estimates, while direct statement and the level of attribute changes in the task design appear to be the strongest predictors. This suggests that in our study, the results are not specific to a certain group but apply to the entire sample.

In conclusion, in this chapter, we did not touch the question of which technique - DCE or CV, is more valid. There is evidence in favor of both of these techniques (e.g. Ryan, 2004a; Ryan and Watson, 2009; Telser and Zweifel, 2007), though some authors may question the theoretical validity of DCE (e.g. Bijlenga et al., 2011; Taylor and Armour, 2002) and show its sensitivity to functional form (e.g. Hanley et al., 1998). Here, we aimed to study the discrepancy in WTP from the two techniques. Systematic differences would eliminate the necessity to choose which method is more valid. So far, our conclusions are: (i) DCE produces systematically higher WTP estimates than people state in CV task; (ii) there is practically no evidence for the consistency between the two estimates; (iii) the difference in WTP estimates from the two techniques mostly results from incertitude in behavior of respondents who place low value on the presented changes, and rises with the increase in the attribute changes under valuation. These conclusions are not specific to any sociodemographic group and hold for the entire sample.

From the methodological perspective, assuming open-ended/payment card CV to be a reference due to its closeness to actual choices (Frew et al., 2003; Ryan and Watson, 2009), 
measures in DCE design reducing this discrepancy should be considered. These measures might include but do not need to be restricted to: non-linear utility function specifications, introduction of an opt-out option in discrete choice tasks, introducing marginal changes rather than total variation change in those tasks where possible, and introduction of a price attribute with plausible levels. In any case, the specific effects of these measures on the convergence in $W T P_{D C E}^{\Delta}$ estimates to directly stated $W T P_{C V}^{\Delta}$ should be further investigated. The part of discrepancy that is brought up by respondents' incertitude in decision making can be addressed by discrimination between choice tasks for respondents by the relevance of the presented attributes to them. Two possible strategies discussed in this chapter are: the indifferent choice option and adaptive choice designs in DCE. 



\section{CHAPTER 5}

Willingness and ability to pay for physician services in six Central and Eastern European countries

Submitted for publication 


\section{Chapter 5}

\section{Abstract}

Background: Patient charges for health care services are implemented in high-income countries to reduce unnecessary service use and to involve consumers in health care funding. Although service use in Central and Eastern Europe (CEE) is high, patient charges for services are not common. Moreover, there is no evidence on the potential consumption effects of service charges in these countries.

Objective: In this chapter, we provide evidence on the potential impact of patient charges on the consumption of specialized physician services in six CEE countries: Bulgaria, Hungary, Lithuania, Poland, Romania, and Ukraine.

Methods: We apply a semi-parametric survival analysis to the stated WATP in order to identify potential demand pools, i.e. the shares of population willing and able to pay a certain fee in case they need a service. Subsequently, we calculate price, income and age semielasticities. Data are collected through a survey held in 2010 among representative samples of about 1000 respondents in each country.

Results: Our results suggest that median WATP in the studied countries ranges from $€ 5.15$ to $€ 12.2$ and the country ranking by WATP follows exactly the country ranking by income level. Low service charges, up to $€ 2.5$ in Bulgaria, Hungary, Lithuania and Romania, and up to $€ 5$ in Poland do not appear to drop many people out of the demand pool.

Conclusions: Low patient payments are an acceptable policy tool in case adequate exemption criteria are effectively applied. The lower payment interval should be studied in more detail for Ukraine, however. Conducting demand analysis based on SP data can provide useful information to design patient payment policies. 


\subsection{Introduction}

The implementation of patient charges for publicly-funded health care services has been often recommended to reduce unnecessary service use and thus, to enhance the efficiency of health care resource utilization (Ros et al., 2000; Rovira et al., 1998). Theoretical and empirical research suggests such charges may bring certain welfare gains but if accompanied by efficient administration and sufficient quality improvements (McPake, 1993; Pavlova et al., 2010). In Central and Eastern European (CEE) countries, where patients often pay for pharmaceuticals and medical devices, as well as informally to providers (Balabanova and McKee, 2002; Belli et al., 2004; Ensor, 2004; Gaál et al., 2010; Gaál and McKee, 2005; Szende and Culyer, 2006; Thompson and Witter, 2000), formal charges for health care services are not very common. This can be explained by the post-socialistic context and the political inability to implement these commonly unpopular measures (Tambor et al., 2011), rather than by real evidence on their potential effects. In fact, evidence on the potential impact of patient charges on the consumption of health care services in CEE countries is lacking.

This chapter focuses on the potential consumption effects of the official patient charges for specialized physician services in six CEE countries, namely Poland and Hungary (developed CEE countries), Bulgaria and Romania (less developed CEE countries), and Lithuania and Ukraine (post-Soviet countries, Ukraine being far less economically developed). The country context is explained in detail in Chapter 1. All of these countries, except for Ukraine, have implemented social health insurance systems. Ukraine has maintained the tax-based Semashko system which claimed universal coverage, where official patient charges are practically illegal except for some specific services.

Bulgaria is the only country in this group, where patient charges for outpatient services (1\% of country minimum wage per visit) effectively exist since 2000 (Atanasova et al., 2011). In the other countries, i.e. Hungary, Lithuania, Poland, and Romania, patient charges are applicable to services outside the packages covered by the social insurance. Hungary introduced official co-payments in 2007 (around $€ 1$ for a visit to a physician) (Baji et al., 2012a), but they were abolished after a national referendum less than 1 year after they were implemented. Outside the official cost-sharing arrangements, all six countries suffer from quasi-formal and informal payments (also in outpatient care). Quasi-formal charges are official charges set up by the health care establishments without clear government regulations. In the context of health care underfunding, these charges allow patients to obtain services with 
quicker access and higher quality, although this is not fully legal (Gaál et al., 2010; Gotsadze and Gaál, 2010; Murauskiene et al., 2010). Informal (under-the-table) patient payments for physician services are also widespread, especially in Ukraine and Romania, but also in the other countries (Stepurko et al., 2011). Thus, patients in all six countries are confronted with various charges when using physician services (Pavlova et al., 2012), but official fees exist only in Bulgaria.

Given the absence of official patient charges for physician services in four of the studied countries, their rather short lifetime in Hungary, and the rather uniform payments in Bulgaria, we base our analysis on SP. CV data from the six CEE countries was collected via national representative surveys in 2010. We analyzed and compare stated WATP for specialized physician services of high quality and quick access. Rather few studies rely upon SP to quantify the potential effects of user fees on health care demand (Skriabikova et al., 2010). We applied a survival analysis, similar to Mataria et al., (2007), to derive the underlying demand pool functions and, consequently, the price and other factor elasticity. In contrast to Mataria et al. (2007), we applied a semi-parametric modeling approach which better fits the data (as it allows for a non-regular shape of the hazard functions). We also make crosscountry comparisons, which has not been done in previous research.

\subsection{Methods}

\subsubsection{Data collection}

We exploited data from national representative household surveys held in July-August 2010 in the six CEE countries: Bulgaria, Hungary, Lithuania, Poland, Romania, and Ukraine. The target samples consisted of about 1000 respondents in each country. The sampling strategy was based on a multi-stage random probability method and is described in Appendix B in detail. The standardized questionnaire contained 6 main blocks, including questions about WATP and socio-demographic characteristics of the respondent and the respondent's household. The design of the contingent valuation task was similar to that in the small scale survey in Ukraine, described in Chapter 3. The main difference was that in this survey, respondents were presented with one valuation task only. In this task, respondents were presented with the scenario of major health symptoms and were asked if they would be willing and able to pay for the visit to a medical specialist provided that the services were of 
good quality and quick access, which was specified in a show card. The exact wording of the task is presented in Appendix D. Those, who were willing to pay, were first asked to choose one of the payment intervals shown on the card to frame the answer and prevent respondents from overstating their preferences. Then, respondents were asked to specify an exact amount within the interval chosen. If a respondent was not able to state the amount exactly, but could define the interval, the midpoint was filled in by the interviewer. The payment card format, as opposed to dichotomous choice, is generally known to produce more conservative estimates (closer to real WTP) and not subject to mid-point bias (Ryan et al., 2004). Exact statement (open-ended format) was added to obtain more complete information from the respondents.

\subsubsection{Model specification}

For the purpose of modeling, we assumed that the WATP level stated by a respondent reflected his/her real WATP. That meant that if a fee for a visit was lower than a person's WATP the person would stay in what we defined as a potential demand pool, while if the fee exceeded the WATP level, a person would drop out of the potential demand pool. The potential demand pool refers to the share of the population which is potentially willing and able to purchase a service in case a certain health problem occurs. Thus, this is demand conditional on having health problems. WATP in our analysis was the counterpart of the time to death variable in a classical survival analysis, and the fee level was analogous to time (further fee is denoted as $t$ ). The empirical problem was to model the hazard of dropping out of the potential demand pool at a certain fee level using information about the respondents' WATP and socio-demographic characteristics.

First, we assumed that the underlying continuous hazard function satisfied the proportional hazard properties. That is, the hazard rate $\theta$ for a person $i$ to drop out at certain fee level $t^{*}$ from the potential demand pool was proportional to certain basic hazard $\theta_{0}$ for this level, with a multiplicative factor depending on personal characteristics $X_{i}$ :

$\theta_{i}(t, X)=\theta_{o}\left(W A T P_{i}=t^{*}\right) \exp \left(\beta X_{i}\right)$

where $\beta$ is a vector of parameter coefficients.

Hazards derived from raw data behaved rather unevenly and differently across countries. Therefore, in order to better fit the data, we applied a semi-parametric specification of the empirical hazard function. For this purpose we split fee levels into a number of intervals $t_{j}$, 
where $j=1,2, \ldots J$. The continuous hazard may be transformed to obtain the function for the interval specific hazard $h_{j}$ (e.g. Hosmer et al., 2008) in a form:

$$
h_{j}(X)=1-\exp \left[-\exp (\beta X) \int_{t \in t_{j}} \theta_{0}(t) d t\right]
$$

where the integral represents the cumulated baseline hazard in the interval $j$. Denote the logarithm of this amount as $\gamma_{j}$. Then, the empirical problem may be transformed into fitting a complementary log-logit model of the interval hazard:

$$
\log \left(-\log \left[1-h_{j}(X) \mid\right)=\beta X+\gamma_{j} \quad \text { (3) }\right)^{2}
$$

\subsubsection{Two-stage approach and intervals}

In this analysis, we did not distinguish between the true zero answers and so called free-riders. However, we admitted that different drivers might underlie the decision of stating a zero or non-zero WTP and the decision about the actual level of the WTP. Therefore, we applied a two-stage modeling approach ${ }^{3}$. That is, independence of the two decision stages was assumed and we parameterized equation 3 two times for each country. The first stage equation included one constant representing the log of the baseline hazard of dropping out of the demand pool if any fee is introduced. The second stage equation contained constants specific for each of the positive payment intervals.

We used the midpoints and the ends of the intervals presented in the payment cards (that helped exact framing of the WATP statement) for the break-down of the fee scale. This determined the step of $€ 2.5$ for the fee scale intervals. However, in different countries spikes in the response frequency appeared just below and above these values, which correspond to the round numbers in local currencies. Thus, all these values should logically be assigned to one interval. Therefore we used slightly shifted bounds in order to incorporate round values around initial bound into the lower interval. Thus we obtained intervals of: $€(0-2.6)$, [2.6-6), [6-7.6), [7.6-11), [11-12.7), [12.7-25], (25 and more]. The last interval is unbounded and,

\footnotetext{
${ }^{2}$ In this specification $\gamma_{j}$ is an interval specific constant, which does not have simple direct interpretation. Observe that $\gamma_{j}=\log \left[\int_{a_{j-1}}^{a_{j}} \theta_{0}(u) d u\right]=\log \left[-\log \left(1-h_{0 j}\right)\right]$, which means that this constant represent logarithmic transformation of cumulated baseline continuous hazard in the interval, or inverse complementary log-logistic transformation of interval (discrete) baseline hazard.

${ }^{3}$ We also tested for continuous decision process specification by estimating joint models with zero fee included as a separate interval. The results differed to some extent in magnitude, but were fully consistent in relative magnitudes and directions of change, leading to similar conclusions. As the continuous specification accounts for questionable zero answers it may understate survival rates. Therefore, we present here the results of a two-stage approach application.
} 
hence, hazard rates are infinite in it (everyone, who stays in the pool till the fee $€ 25$, drops out of it anyway). Therefore, it was plausible to treat respondents expressing WATP in the last interval as censored. Consequently, there are no constants corresponding to the last interval in the models and no predictions were done based on it ${ }^{4}$.

\subsubsection{Predictions}

The derivation of the survival functions (potential demand pool functions) for each country in the study was further done based on the predicted interval hazards. The prediction of the hazard rates was done using the parameterization of equation 3. For this purpose, sociodemographic characteristics were held at average sample levels within each country. Thus, the survival rate for interval $j$ was calculated as:

$$
S_{j}=\prod_{k=1}^{j}\left(1-h_{k}\right)=\prod_{k=1}^{j}\left(1-\left(1-\exp \left(-\exp \left(\underset{\text { country }}{\mathrm{E}}[\hat{\beta} X]+\gamma_{k}\right)\right)\right)\right)
$$

\subsubsection{Price elasticity}

The price elasticity (i.e. with regards to fee level $t$ ) in the semi-parametric specification does not make much sense in its full form, because the baseline hazard is actually a non-continuous step function consisting only of the combination of interval specific constants. The percentage change in the survival rate moving from interval to interval is of interest. This can be interpreted as semi-elasticity with regard to a one interval change in price (user fee).

Thus, the semi-elasticity across intervals (moving from one interval to the next one) were written as:

$$
\partial \log \left[S\left(t_{j}, X\right)\right] / \partial t=\partial \log \left[S\left(a_{j}, X\right)\right]_{t_{j-1}}^{t_{j}}=-\exp (\beta X)\left[\exp \left(\gamma_{j}\right)\right]
$$

It is notable that the price semi-elasticity depends on population characteristics. We applied the same approach as for predicting survival functions, i.e. we used average sample predictions $\mathrm{E}_{\text {country }}(\beta X)$ for further calculations, thereby relating estimates to the current country context. For presentation purposes, we only calculated the price semi-elasticity of

\footnotetext{
${ }^{4}$ Intuitively, predictions for the last interval are obvious: interval hazard should be equal to 1 , and survival rate to $0 \%$.

${ }^{5}$ Given properties of the survival function we obtain:$$
\log \left[S\left(t_{j}, X\right)\right]=-\int_{0}^{t_{j}} \theta(t, X) d t=-\exp (\beta X) \int_{0}^{t_{j}} \theta_{o}(t) d t=-\exp (\beta X) \sum_{0}^{t_{j}} \exp \left(\gamma_{j}\right)
$$$$
\text { Substituting for difference in logarithms of survival function in equation } 5 \text { gives the presented result. }
$$ 


\section{Chapter 5}

survival when moving between neighboring intervals. Estimations between more distant intervals would not be precise.

\subsubsection{Elasticity by covariates}

We applied the same framework with regards to covariates, and estimate a semi-elasticity for them, which represents the percent change in the survival function in response to a unit change in the covariate of interest $x$. It is calculated as:

$$
\partial \log \left[S\left(t_{j}, X\right)\right] / \partial x=-\beta_{x} \exp (\beta X) \int_{0}^{t_{j}} \theta_{o}(t) d t=-\beta_{x} \exp (\beta X) \sum_{k=0}^{j} \exp \left(\gamma_{k}\right)
$$

Note that the survival (potential demand pool) elasticity with regards to $x$-s depends on the price interval, or cumulative hazard in it, as well as on the level of $x$. Additionally, it depends on the level of all other characteristics, which we set to average sample levels $\mathrm{E}_{\text {country }}(\beta X)$. Thus, for each characteristic we may obtain a different elasticity per interval.

\subsection{Results}

\subsubsection{Descriptive statistics of the data}

Descriptive statistics of the samples in the study are presented in Table B.2 in Appendix B. Their age and gender composition is reasonably close to that of the adult population in the studied countries according to national statistics. The proportion of respondents, who express a positive WATP for specialized physician services provided that they are of high quality and quick access, is rather high in all six countries. The most positive about paying for the services are Romanians and Lithuanians (81.43\% and 80.90\%), followed by Bulgarians (75.96\%). Substantially lower shares of Ukrainian and Polish respondents $(73.47 \%$ and 72.96\%) state a positive WTP. The lowest share is in Hungary (66.54\%).

Even if zero answers are accounted for, the level of the mean and median WATP in all six countries are rather substantial. Accounting for only positive values does not change the

\footnotetext{
${ }^{6}$ Semi-elasticity with regards to exponential of linear combination of characteristics $\mathrm{X}$ can be written as: $\partial \log \left[S\left(a_{j}, X\right)\right] / \partial \exp (\beta X)=-\int_{0}^{a_{j}} \theta_{j o}(t) d t$

However, $\partial \exp (\beta X)$ can be rewritten as $\beta_{x} \exp (\beta X) \partial x$ in order to relate it to change is characteristic $x$. Substituting in the previous equation we obtain equation 6 .
} 
pattern much. In Poland, the level of WATP is the highest, while Ukraine is the very last by this indicator. Hungary and Lithuania share the second place in the chart of WATP levels. However, in Hungary this level becomes substantially higher if only positive values are considered, which is due to a large number of zero answers. In Romania, the mean WATP level is higher than in Bulgaria but, they are close in median levels. Country ranking by mean and median positive WATP level follows perfectly their ranking by income, and still closely if zeros are considered (except for Hungary).

\subsubsection{WATP modeling}

The results of the two-stage modeling of the hazard rates of dropping out from the potential demand pool based on the stated WATP are presented in Table 5.1. For each country, the first column represents the hazard model conditional on having a positive WATP (second stage decision), while the second column is the hazard rate (probability) of having a zero WATP (first stage decision). The models have theoretically valid coefficients, i.e. elderly and people with lower income are expected to express lower WATP. However, the hazard function at positive fee levels for Ukraine does not pass the likelihood-ratio test against the constant only model and, hence, covariates in the model have rather imprecise or no effect on the WATP level. Baseline hazards follow a common pattern for all six countries. They are the lowest at the interval corresponding to the fee level of $€ 0-2.5$. Then, they gradually but unevenly increase. It is very common that hazards behave unstable at the higher levels of WATP due to fewer observations at those levels.

For those who state a positive WATP, age is not associated with WATP changes except for Ukraine, where the association is small in magnitude and bordering on statistical significance. Age plays a more important role for increased hazards of stating zero WATP in Romania and Ukraine, but also for Poland and Lithuania.

In Bulgaria, Hungary, Lithuania, and Romania, income is a significant factor at both decision stages. However, in Hungary, the magnitude of its effect is similar at both decision stages, while in the other three countries the effect on the hazard rate of stating a zero WATP is much higher than for the WATP level. In Poland, income defines only hazards at the first decision point (zero/positive WATP) while in Ukraine, it is not significant at any stage. Household size was introduced in order to weight the effect of total household income, and it turns out to be significant only in Poland and Hungary. 
Table 5.1 Two-stage hazard models based on stated WATP

\begin{tabular}{|c|c|c|c|c|c|c|c|}
\hline \multirow{2}{*}{ Effect } & & \multicolumn{2}{|c|}{ Bulgaria } & \multicolumn{2}{|c|}{ Hungary } & \multicolumn{2}{|c|}{ Lithuania } \\
\hline & & $\begin{array}{l}\text { At pos. } \\
\text { fee }\end{array}$ & $\begin{array}{c}\text { At zero } \\
\text { fee }\end{array}$ & $\begin{array}{l}\text { At pos. } \\
\text { fee }\end{array}$ & $\begin{array}{l}\text { At zero } \\
\text { fee }\end{array}$ & $\begin{array}{l}\text { At pos. } \\
\text { fee }\end{array}$ & $\begin{array}{l}\text { At zero } \\
\text { fee }\end{array}$ \\
\hline \multirow[t]{2}{*}{ Age } & \multirow{2}{*}{ years } & 0.005 & 0.007 & 0.003 & 0.005 & 0.005 & $0.013^{* *}$ \\
\hline & & $(0.003)$ & $(0.006)$ & $(0.003)$ & $(0.004)$ & $(0.003)$ & $(0.006)$ \\
\hline \multirow[t]{2}{*}{ Income } & \multirow[t]{2}{*}{$€$ thds. } & $-0.416 * *$ & $-2.238 * * *$ & $-0.526 * * *$ & $-0.508 * *$ & $-0.370 * * *$ & $-1.502 * * *$ \\
\hline & & $(0.199)$ & $(0.574)$ & $(0.178)$ & $(0.230)$ & $(0.104)$ & $(0.338)$ \\
\hline \multirow{2}{*}{\multicolumn{2}{|c|}{ Size of the household }} & 0.035 & 0.035 & -0.009 & 0.029 & -0.008 & 0.053 \\
\hline & & $(0.040)$ & $(0.067)$ & $(0.038)$ & $(0.050)$ & $(0.036)$ & $(0.071)$ \\
\hline \multirow[t]{3}{*}{ Sex } & \multirow{3}{*}{$\begin{array}{l}\text { male } \\
\text { female }\end{array}$} & ref. & ref. & ref. & ref. & ref. & ref. \\
\hline & & $0.150 *$ & 0.006 & $0.203 * *$ & -0.14 & -0.043 & $-0.551 * * *$ \\
\hline & & $(0.087)$ & $(0.150)$ & $(0.091)$ & $(0.115)$ & $(0.083)$ & $(0.157)$ \\
\hline \multirow[t]{5}{*}{ Residence } & \multirow[t]{2}{*}{ village } & $0.262 * *$ & -0.152 & $0.202 * *$ & 0.067 & -0.145 & 0.001 \\
\hline & & $(0.103)$ & $(0.170)$ & $(0.104)$ & $(0.135)$ & $(0.094)$ & $(0.181)$ \\
\hline & \multirow{3}{*}{$\begin{array}{l}\text { small cities } \\
\text { big cities }\end{array}$} & ref. & ref. & ref. & ref. & ref. & ref. \\
\hline & & $0.290 * * *$ & $0.346 *$ & $0.843^{* * *}$ & $0.392 * * *$ & $0.285^{* * *}$ & $0.445 * *$ \\
\hline & & $(0.112)$ & $(0.207)$ & $(0.127)$ & $(0.148)$ & $(0.099)$ & $(0.195)$ \\
\hline \multirow[t]{5}{*}{ Education } & \multirow[t]{2}{*}{ no or primary } & 0.182 & $0.595^{* * *}$ & $0.212 * *$ & 0.091 & -0.026 & 0.136 \\
\hline & & $(0.123)$ & $(0.172)$ & $(0.106)$ & $(0.136)$ & $(0.138)$ & $(0.222)$ \\
\hline & \multirow{3}{*}{$\begin{array}{l}\text { secondary } \\
\text { tertiary }\end{array}$} & ref. & ref. & ref. & ref. & ref. & ref. \\
\hline & & $-0.211^{* *}$ & $-0.707 * * *$ & 0.182 & -0.197 & -0.023 & -0.241 \\
\hline & & $(0.107)$ & $(0.269)$ & $(0.130)$ & $(0.188)$ & $(0.087)$ & $(0.174)$ \\
\hline \multirow{2}{*}{\multicolumn{2}{|c|}{$\begin{array}{l}\text { Number of family members } \\
\text { with chronic disease }\end{array}$}} & -0.012 & 0.092 & 0.107 & $-0.293 * * *$ & -0.054 & -0.072 \\
\hline & & $(0.070)$ & $(0.123)$ & $(0.073)$ & $(0.101)$ & $(0.065)$ & $(0.128)$ \\
\hline Chronic & diabetes & 0.18 & -0.241 & $-0.350 * *$ & 0.257 & $-0.406^{*}$ & 0.37 \\
\hline states & & $(0.163)$ & $(0.271)$ & $(0.163)$ & $(0.193)$ & $(0.211)$ & $(0.282)$ \\
\hline & CVD & -0.116 & 0.123 & -0.146 & 0.166 & -0.143 & 0.265 \\
\hline & & $(0.121)$ & $(0.201)$ & $(0.119)$ & $(0.150)$ & $(0.107)$ & $(0.192)$ \\
\hline & kidney, liver, & 0.006 & 0.178 & 0.305 & -0.301 & -0.02 & 0.242 \\
\hline & lung & $(0.154)$ & $(0.223)$ & $(0.187)$ & $(0.288)$ & $(0.139)$ & $(0.219)$ \\
\hline & other chronic & 0.086 & 0.084 & -0.023 & $0.416^{* * *}$ & $0.205 * *$ & 0.005 \\
\hline & & $(0.116)$ & $(0.185)$ & $(0.127)$ & $(0.158)$ & $(0.102)$ & $(0.190)$ \\
\hline Past use & visited & $-0.278 * *$ & -0.355 & 0.143 & -0.12 & -0.047 & -0.078 \\
\hline experience & physician & $(0.138)$ & $(0.248)$ & $(0.124)$ & $(0.143)$ & $(0.097)$ & $(0.189)$ \\
\hline & paid officially & $0.290 * *$ & 0.125 & -0.129 & $-0.642 * *$ & $-0.220 * *$ & -0.285 \\
\hline & & $(0.116)$ & $(0.201)$ & $(0.141)$ & $(0.282)$ & $(0.099)$ & $(0.207)$ \\
\hline & paid & 0.117 & $-0.564^{*}$ & -0.146 & $-1.065^{* * *}$ & 0.038 & $0.372 *$ \\
\hline & informally & $(0.159)$ & $(0.334)$ & $(0.117)$ & $(0.227)$ & $(0.117)$ & $(0.216)$ \\
\hline Interval & $€[0+]$ & & $-0.977 * *$ & & $-0.525^{*}$ & & $-1.499 * * *$ \\
\hline constant & & 0.000 & $(0.456)$ & 0.000 & $(0.283)$ & 0.000 & $(0.396)$ \\
\hline & $€\left(0-2.5^{+}\right]$ & $-2.282 * * *$ & & $-3.112 * * *$ & & $-2.883 * * *$ & \\
\hline & & $(0.269)$ & & $(0.276)$ & & $(0.249)$ & \\
\hline & $€[2.6-5+]$ & $-1.953 * * *$ & & $-2.059 * * *$ & & $-1.481 * * *$ & \\
\hline & & $(0.266)$ & & $(0.248)$ & & $(0.214)$ & \\
\hline & $€[6-7.5+]$ & $-1.126 * * *$ & & $-1.474 * * *$ & & $-1.401 * * *$ & \\
\hline & & $(0.257)$ & & $(0.243)$ & & $(0.217)$ & \\
\hline & $€[7.6-10+]$ & -0.346 & & $-1.101 * * *$ & & $-0.833^{* * *}$ & \\
\hline & & $(0.256)$ & & $(0.243)$ & & $(0.214)$ & \\
\hline & $€[11-12.5+]$ & $-0.520^{*}$ & & $-1.776 * * *$ & & $-0.694 * * *$ & \\
\hline & & $(0.278)$ & & $(0.271)$ & & $(0.219)$ & \\
\hline & $€[12.7-25]$ & $0.663 * *$ & & -0.315 & & -0.113 & \\
\hline & & $(0.299)$ & & $(0.245)$ & & $(0.222)$ & \\
\hline Number of c & ervations & 2,061 & 817 & 2,326 & 934 & 2,974 & 951 \\
\hline Log-likeliho & & $-1,055.77$ & -394.04 & $-1,092.67$ & -560.73 & $-1,424.86$ & -426.34 \\
\hline Wald test (c & & $706.89 * * *$ & $102.86 * * *$ & $859.59 * * *$ & $89.32 * * *$ & $1069.81^{* * *}$ & $75.90 * * *$ \\
\hline Likelihood I & o test (chi2) & $45.87 * * *$ & $98.16^{* * *}$ & $67.53 * * *$ & $85.82 * * *$ & $44.24 * * *$ & $60.4^{* * *}$ \\
\hline
\end{tabular}

Significance: $* p<0.1 ; * * p<0.05 ;{ }^{* * *} p<0.01$; $\quad$ ref. - reference group $\quad$ (continued on the next page) 
Table 5.1 (continued) Two-stage hazard models based on stated WATP

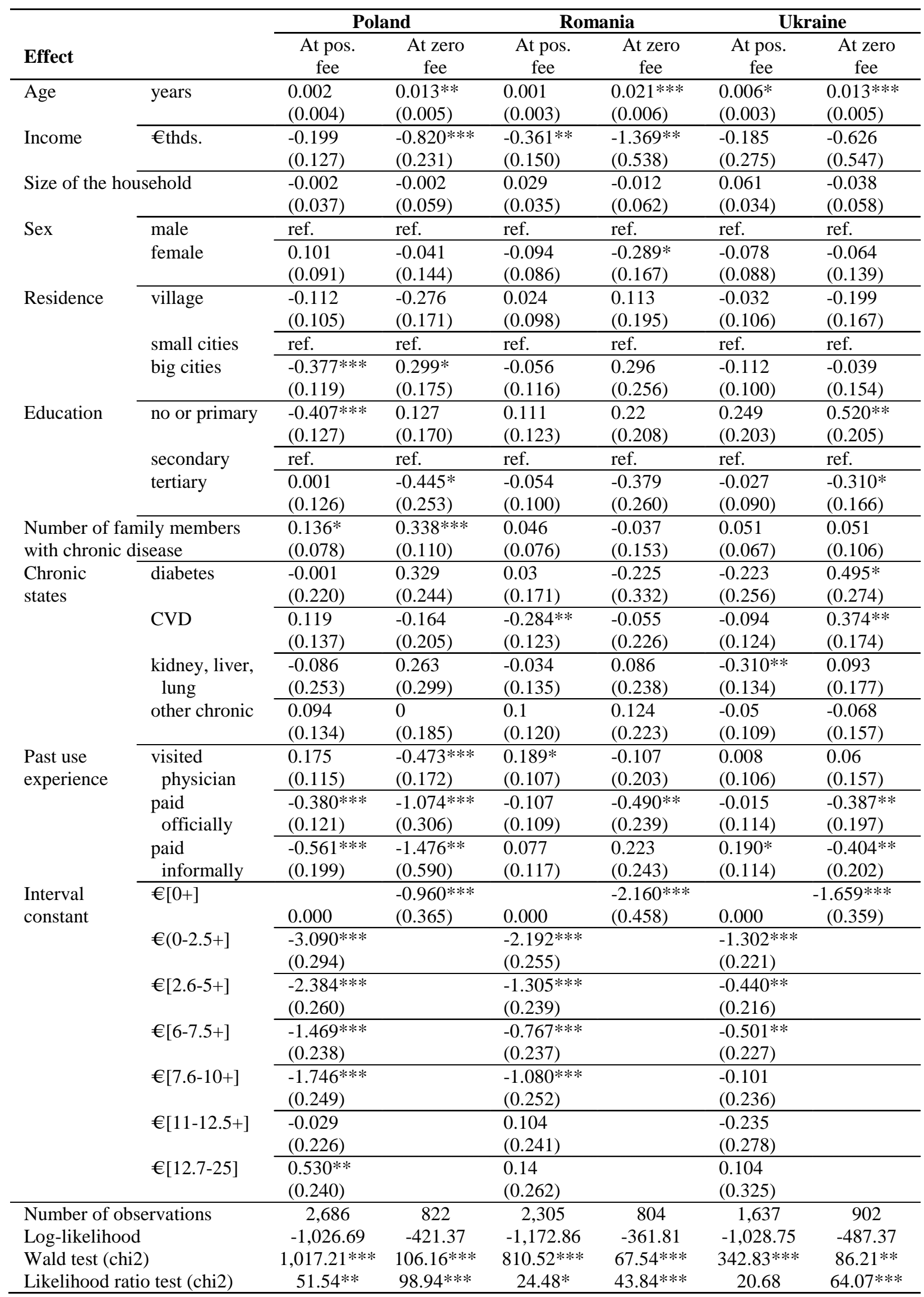

Significance: $* p<0.1$; ${ }^{* *} p<0.05$; $* * * p<0.01$; $\quad$ ref. - reference group 
Place of residence is an important demand pool factor mostly at positive WATP levels, but also at the first stage decision, in Bulgaria, Hungary, Lithuania, and Poland. Level of education demonstrates a differential effect in different countries. It is, however, most important for the first stage decision in Bulgaria, less in Ukraine, where a higher education level is associated with higher chances of having a positive WATP.

Chronic diseases affect the valuation of specialized physician services. Having diabetes in Lithuania and Hungary, cardio-vascular disease (CVD) in Romania, and kidney, liver, or lung problems in Ukraine are associated with higher WATP values and, thus, lower hazards of dropping out of the potential demand pool at any fee level. These effects are expected. However, having another chronic disease is associated with lower WATP levels in Lithuania, and with higher chances of stating zero WATP in Hungary. CVD is also associated with higher chances of zero WATP in Ukraine. These effects are not directly expected. However, they might be caused by interfering income effects. This implies that people with these disease have a lower income and, hence, express lower WATP levels.

Finally, past use and payment experiences have differential effects throughout the studied countries. Those who visited a physician during the past year have a higher chance of stating a positive WATP in Poland while in Bulgaria they express a lower WATP. In Romania physician visits increase WATP. Past experience in paying either officially or informally increases the chances of having zero WATP in Hungary, Poland, and Ukraine. In Poland this experience is also associated with a lower WATP and, thus, a higher chance to drop out of the demand pool at any fee level. Lithuanians state a higher WATP if they have experience in paying officially in the last year, while in Bulgaria the effect is the opposite. Opposite effects of informal payment experience are observed in these countries: in Bulgaria, it is associated with higher chances of positive WATP while in Lithuania - with lower chances. In Romania, official payment experience is associated with higher probability of stating positive WATP.

Figure 5.1 presents the survival models (potential demand pools) built based on the two-stage modeling framework. Panel a) represents potential demand pool functions conditional not only on having health problems, but also on being positive about paying, while in panel b) the demand pool is unconditional of zero or positive WATP, but is still conditional on having health problems that make one go to a medical specialist. The zero fee interval in panel b) represents some very small increases in fee levels, i.e. increases close to zero. The survival rate at this interval represents the mean predicted proportion of respondents who state they are 
willing to pay any fee. Both approaches produce survival rates close to the raw proportions from the data with a slightly overstated survival rates in the zero interval.

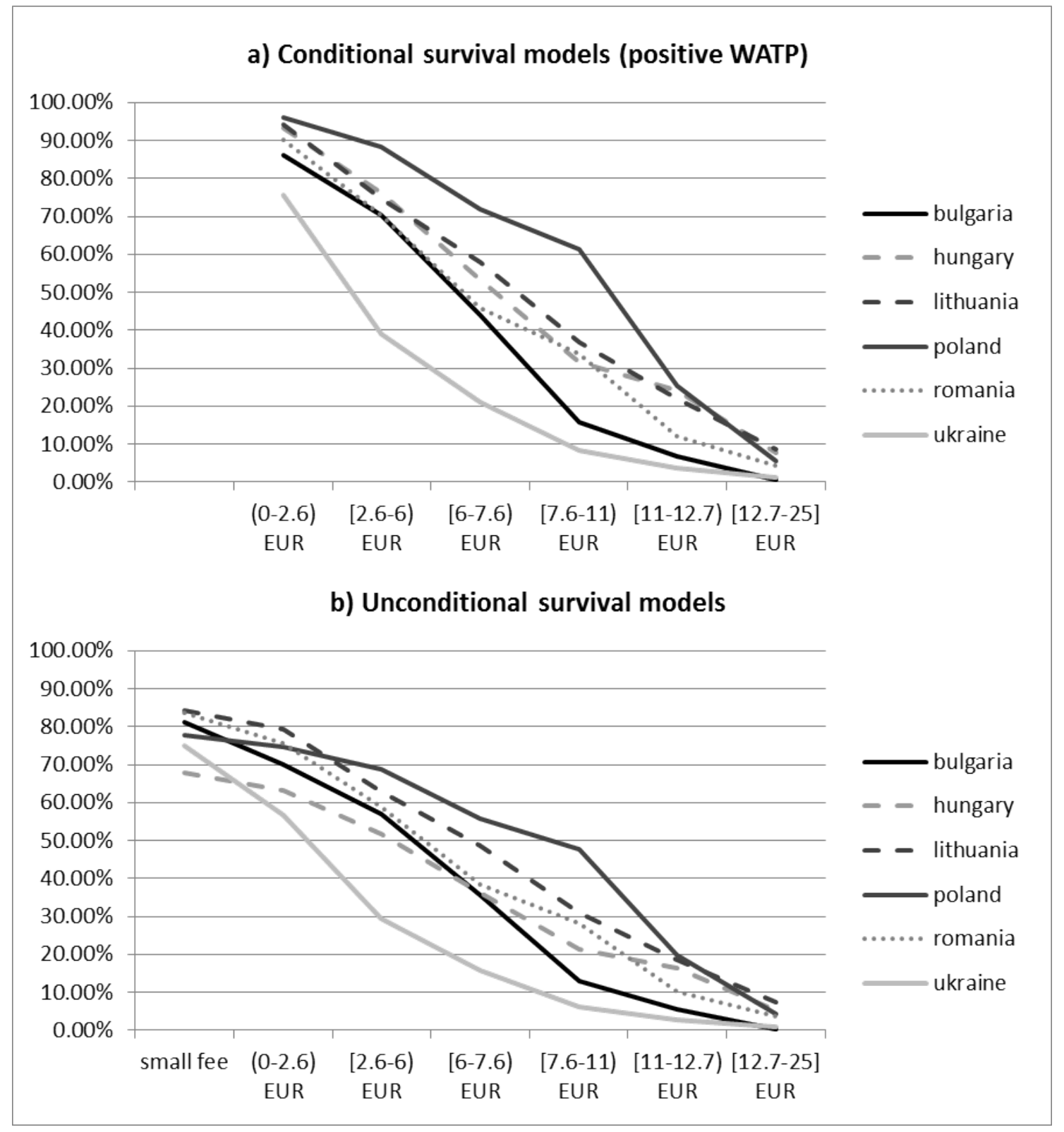

Figure 5.1 WATP survival models (potential demand pools) based on two-stage approach to hazard modeling.

Because of the high proportion of respondents stating that they are not willing or able to pay for specialized physician services, the unconditional potential demand pool in panel b) for Poland and Hungary is shifted downward especially at lower fee levels. We find that this 
underestimates the real demand pool potential in these countries. Therefore, we consider the conditional demand pools as the ones that better reflect reality.

Poland differs from other countries by having the highest potential demand pool function, while Ukraine has the lowest. Hungary, Lithuania, and Romania have rather close potential demand pool dynamics. Finally, the demand pool in Bulgaria is close to the middle for the six countries at the lower fee levels, and is the most elastic between 5 and $€ 10$ fee. After $€ 10$ it becomes less elastic and approaches the one in Ukraine after $€ 12.5$ fee.

\subsubsection{Demand pool elasticities}

The calculated values for the price, income and age semi-elasticities of the demand pool are presented in Table 5.2. At the lowest fee interval, i.e. between $€ 0$ and $€ 2.5$, the demand pool is relatively inelastic in most countries. Most of the drop out at such fee level is in Ukraine (27.8\%), Bulgaria (14.7\%) and Romania (10.3\%). The least elastic demand pool at this fee interval is observed in Poland and Lithuania, a little bit more in Hungary. The increase to the next fee interval, i.e. between $€ 2.5$ and $€ 5$, causes a very large drop from the demand pool in Ukraine (65.9\%). In Bulgaria, Hungary, Lithuania, and Romania, this fee level causes dropouts at the level of around 20-25\%, which can be already considered as rather substantial. Only in Poland a fee increase to this interval does not cause many respondents to drop-out as the semi-elasticity for moving to this interval is around $-8.3 \%$. Up till $€ 10$, Poland has the least elastic demand pool function among the six countries. However, a further fee increase of $€ 5$ makes the demand pools in all countries more elastic and causes substantial drop-outs, especially in Ukraine and Bulgaria.

The income semi-elasticity estimates show that the demand pool is rather weakly affected by income at low fee levels for respondents with positive WATP. This is especially true for Poland, but also for Lithuania, Hungary and Romania. In Poland, income continues to have the weakest effect through all fee levels. For other countries the income elasticity of potential demand follows a similar pattern in all fee intervals up to $€ 7.5$. The most responsive to income is the survival function in Bulgaria starting from a $€ 7.5$ fee, and in Hungary in the interval $€ 6-€ 10$. In Romania, the income elasticity starts to increase rapidly after a $€ 10$ fee. In Ukraine and Lithuania, the income elasticity increase remains relatively stable throughout all fee intervals which may also be interpreted as the weak impact of income on the WATP statement. At the first stage decision (i.e. stating zero or positive WATP), the biggest effect of 
income is observed in Bulgaria (46.9\%), less in Lithuania (25.88\%) and Romania (24.4\%), even less in Hungary (19.78\%) and Ukraine (18.08\%).

Table 5.2 Price and other factor semi-elasticity of potential demand pools

\begin{tabular}{|c|c|c|c|c|c|c|c|}
\hline & & \multicolumn{6}{|c|}{ Semi-elasticity } \\
\hline & & Bulgaria & Hungary & Lithuania & Poland & Romania & Ukraine \\
\hline \multicolumn{8}{|l|}{ From } \\
\hline No fee & Small fee & $-21.0 \%$ & $-38.9 \%$ & $-17.2 \%$ & $-25.0 \%$ & $-17.8 \%$ & $-28.9 \%$ \\
\hline Small fee & $€(0-2.5+]$ & $-14.7 \%$ & $-7.0 \%$ & $-5.8 \%$ & $-4.1 \%$ & $-10.3 \%$ & $-27.8 \%$ \\
\hline$€(0-2.5+]$ & $€[2.6-5+]$ & $-20.4 \%$ & $-20.0 \%$ & $-23.5 \%$ & $-8.3 \%$ & $-24.9 \%$ & $-65.9 \%$ \\
\hline$€[2.6-5+]$ & $€[6-7.5+]$ & $-46.7 \%$ & $-35.9 \%$ & $-25.5 \%$ & $-20.8 \%$ & $-42.7 \%$ & $-62.0 \%$ \\
\hline$€[6-7.5+]$ & $€[7.6-10+]$ & $-102.0 \%$ & $-52.2 \%$ & $-45.0 \%$ & $-15.7 \%$ & $-31.2 \%$ & $-92.5 \%$ \\
\hline$€[7.6-10+]$ & $€[11-12.5+]$ & $-85.7 \%$ & $-26.6 \%$ & $-51.7 \%$ & $-87.6 \%$ & $-101.9 \%$ & $-80.9 \%$ \\
\hline$€[11-12.5+]$ & $€[12.7-25]$ & $-279.7 \%$ & $-114.6 \%$ & $-92.5 \%$ & $-153.2 \%$ & $-105.7 \%$ & $-113.6 \%$ \\
\hline \multicolumn{8}{|c|}{ Income semi-elasticity (by $€$ 1000) } \\
\hline \multirow[t]{7}{*}{ At: } & Small fee & $46.9 \%$ & $19.8 \%$ & $25.9 \%$ & $20.5 \%$ & $24.4 \%$ & $18.1 \%$ \\
\hline & $€(0-2.5+]$ & $6.1 \%$ & $3.7 \%$ & $2.1 \%$ & $0.8 \%$ & $3.7 \%$ & $5.2 \%$ \\
\hline & $€[2.6-5+]$ & $14.6 \%$ & $14.2 \%$ & $10.9 \%$ & $2.5 \%$ & $12.7 \%$ & $17.3 \%$ \\
\hline & $€[6-7.5+]$ & $34.1 \%$ & $33.1 \%$ & $20.3 \%$ & $6.6 \%$ & $28.1 \%$ & $28.8 \%$ \\
\hline & $€[7.6-10+]$ & $76.5 \%$ & $60.6 \%$ & $36.9 \%$ & $9.7 \%$ & $39.4 \%$ & $45.9 \%$ \\
\hline & $€[11-12.5+]$ & $112.1 \%$ & $74.6 \%$ & $56.1 \%$ & $27.2 \%$ & $76.2 \%$ & $60.9 \%$ \\
\hline & $€[12.7-25]$ & $228.5 \%$ & $134.8 \%$ & $90.3 \%$ & $57.7 \%$ & $114.3 \%$ & $81.9 \%$ \\
\hline \multicolumn{8}{|c|}{ AGE semi-elasticity (by 10 years) } \\
\hline \multirow[t]{7}{*}{ At: } & Small fee & $-1.5 \%$ & $-1.9 \%$ & $-2.2 \%$ & $-3.3 \%$ & $-3.7 \%$ & $-3.8 \%$ \\
\hline & $€(0-2.5+]$ & $-0.7 \%$ & $-0.2 \%$ & $-0.3 \%$ & $-0.1 \%$ & $-0.1 \%$ & $-1.7 \%$ \\
\hline & $€[2.6-5+]$ & $-1.8 \%$ & $-0.8 \%$ & $-1.5 \%$ & $-0.2 \%$ & $-0.4 \%$ & $-5.6 \%$ \\
\hline & $€[6-7.5+]$ & $-4.1 \%$ & $-1.9 \%$ & $-2.7 \%$ & $-0.7 \%$ & $-0.8 \%$ & $-9.3 \%$ \\
\hline & $€[7.6-10+]$ & $-9.2 \%$ & $-3.5 \%$ & $-5.0 \%$ & $-1.0 \%$ & $-1.1 \%$ & $-14.9 \%$ \\
\hline & $€[11-12.5+]$ & $-13.5 \%$ & $-4.3 \%$ & $-7.6 \%$ & $-2.7 \%$ & $-2.1 \%$ & $-19.8 \%$ \\
\hline & $€[12.7-25]$ & $-27.5 \%$ & $-7.7 \%$ & $-12.2 \%$ & $-5.8 \%$ & $-3.2 \%$ & $-26.6 \%$ \\
\hline
\end{tabular}

Age has a rather limited effect on the demand pool at low fee levels. Table 5.2 provides semielasticities of the demand pool by 10 year changes in age. The magnitude of the estimates is rather moderate at all fee levels, except for the highest. The highest effect on the demand pool has age in Ukraine, and to a lesser extent in Bulgaria. Overall, at lower fee intervals (up to $€ 5)$ age differences do not cause many disparities in health care service consumption in all countries under consideration except for Ukraine.

\subsection{Discussion and conclusion}

In this study we exploit a stated WATP measure to study the potential impact of the different patient payment levels on the consumption ability of specialized physician services in six CEE countries. For this purpose a semi-parametric survival analysis was applied. This was 
suggested in a study by Mataria et al. (2007) who used a more restrictive parametric approach. Results of our study apply to the integrated WATP for a visit to a physician, not to separate quality and access characteristics as in mentioned study. They are also limited to the specialists' services with high quality and quick access in cases of major symptoms. The former focus bridges the idea of revenue collection with necessary investments in quality and access of the services. The major symptom scenario only indicates that in case of minor symptoms, the consumption might be lower as more people would prefer alternative (no or self-treatment).

We observe rather substantial levels of WATP in the countries which are comparable to the cost of the services (although the notion of actual service cost is absent in most of these countries). For Bulgaria, Lithuania, and Romania, we use tariffs established in contracts between the National Health Insurance Funds and Provider representatives ${ }^{7}$ for 2012 and bring them to the August 2010 values applying consumer price indexes using national statistics $^{8}$. In Bulgaria, patients additionally pay a co-payment of around $€ 1$ (Atanasova et al. 2011), and thus, the full (time-adjusted) cost for society of an outpatient visit to a specialist would be $€ 9.20$ for the $1^{\text {st }}$ visit and $€ 5.32$ for the follow-ups. In Lithuania, the time adjusted cost of the similar visit varies between $€ 10.30$ and $€ 12.63$ depending on the specialty. In Romania, the basic tariff for examination is around $€ 7.2$, though it varies substantially between specialties and types of providers and in private facilities starts from $€ 14$. For Hungary, we use 2009 cost estimates by Gulácsi et al. (2012), which is approximately $€ 6.8$ for a visit to a medical specialist when figures are adjusted to August $2010^{9}$. For Poland, we use informal sources, such as internet forums, which provide information that in 2011 the cost of a visit (time-adjusted ${ }^{10}$ ) to a medical specialist for uninsured people varied across different regions between $€ 12.25$ (50 zloty) and $€ 17.15$ (70 zloty) with $€ 14.7$ (60 zloty) being the average. For Ukraine, no data about cost of services are available.

Comparing the indicators of the actual cost of services to mean and median WATP levels (zero answers not considered), it can be said that only in Hungary and Romania, people on

\footnotetext{
${ }^{7}$ Data extracted from the official websites of National Insurance Funds of Bulgaria (National Health Insurance Fund of Bulgaria, 2011), Lithuania (Vilnius Territorial Insurance Fund, 2012), and Romania (National Health Insurance Fund of Romania, 2011).

${ }^{8}$ Consumer Price Index for these countries is obtained from National statistics services of Bulgaria (National Statistical Institute of Bulgaria, 2012), Lithuania (Statistics Lithuania, 2012); for Romania we exploit World Bank data (The World Bank, 2012)

${ }^{9}$ CPI for Hungary extracted from OECD data (OECD, 2012b)

${ }^{10}$ CPI for Poland extracted from OECD data (OECD, 2012b)
} 
average value specialists' services substantially higher than their cost to society in the public system. In Bulgaria, the same applies to follow-up visits, while cost of the $1^{\text {st }}$ visit is slightly higher than Bulgarians are willing and able to pay on average for it. In Lithuania and Poland, mean WATP levels lie somewhere in the middle of the range of service cost for these countries, though median values do not even cover the minimal cost. Given that in this study we estimate rough average WATP without service specification, it appears that in these countries cost of services is maximally approached to the socially acceptable levels. This comparison indicates that there is some potential for the implementation of user fees for physician services in the countries.

The survival analysis describes the patterns of the population shares staying in the potential demand pool after a certain fee level and their differences across population groups. In general, the location of the demand curves and their elasticity is consistent with theoretical expectations: i.e. they follow the country ranking by income rather well (The World Bank, 2013). The only exception is the Hungarian unconditional demand curve, which is low due to the high levels of zero answers.

An unresolved question in the analysis is whether zero answers represent true opt-outs when patient fees are introduced. In principle, a true zero WATP is different from protest answers (Mitchell and Carson, 1989; Shackley and Donaldson, 2002). That is if patient payments are implemented, those who state zero WATP will not necessarily forgo services. In this study, we do not make a distinction between protest and true zero WATP answer. Thus, we include all zero answers in the analysis. Hence, unconditional demand curves might be too conservative, i.e. overestimate the share of drop-outs at the first stage: introduction of any fee, and consequently at all other fee levels.

If the fee levels are increased to the actual service cost, this will lead to many drop-outs. In our models, 70-71\% of Bulgarians are willing and able to pay the cost of the follow-up visit, and only $15-18 \%$ - for the first visit. In Hungary, around 54\% of the population will stay in the potential demand pool, in Lithuania - only 24\%, in Poland - between 5.5\% and 25.5\%, depending on the exact level. Cost-sharing is the more feasible option in this case. For example, the current co-payment of $€ 1$ corresponds to $86.5 \%$ of individuals being willing and able to pay in our model (though the level might be higher as the payment interval $€ 0-2.5$ is rather broad).

It should be noted that the demand curves obtained in our analysis, do not give a direct answer to the question of what level of user fees or co-payments is considered to be optimal. This 
depends not only on the potential demand curves, but also on the marginal cost of the services and their quality improvements, as well as on the policy aims. Overall, it is a question of the best trade-off between coverage and quality which maximizes welfare gains (McPake, 1993). The overall framework for conducting such analysis is lacking. However, our potential demand curves provide a good indication of the population groups and shares that will be most problematic and will require exemption/reduction mechanisms at each fee level.

Our results are not fully comparable to the results of other studies due to the semi-parametric specification. We were able to estimate semi-elasticities of the survival (potential demand pool) functions. Translation of the semi-elasticity into a full elasticity (by dividing them by the percentage change in the fee level using the mid-point as a basis) does not provide precise point estimates as the fee scale was initially split into discrete intervals. Nonetheless, such imprecise calculations show that the price elasticity in the lowest payment interval $€ 0-2.5$ varies from -0.08 for Poland to -0.57 in Ukraine. In the second payment interval, the estimates range from -0.5 for Poland to -3.95 for Ukraine. For other countries, the elasticities are in the order of -1.2 to -1.5 . In the fee intervals higher than $€ 5$, the price elasticity increases to a much higher level. Thus, for low fee levels (up to $€ 5$ ) our results for the five CEE countries (excluding Ukraine) are rather consistent with the previous literature based on cross-sectional data and experimental designs in developed countries (Manning et al., 1988; Newhouse and Group, 1993). The Ukrainian population is much more responsive to a fee introduction/increase in the indicated interval, which is comparable to the demand elasticity found in the less developed countries (Ching, 1995; Gertler and van der Gaag, 1990; Mocan et al., 2004).

The socio-demographic differences that we observe are rather consistent with the theoretical expectations and with the previous literature. First of all, income has an expected positive impact on the demand in five of the studied countries. The only country where income has no effect is Ukraine. We explain this by the historical and institutional peculiarities of Ukraine. The well-established payment practices (informal and quasi-formal payments) (Gryga et al., 2010) might eliminate some variation in expressed WATP levels due to common ideas in society about a fair price. We explain the tendency among elderly people to report zero WATP more often by ideological reasons. Some surveys in post-communist countries reveal that nowadays there is a substantial share of people who are nostalgic about the USSR, and this is substantially higher among older groups (Survey, 2011). These ideas are explained by the conviction that social security for older people was more extensive in the USSR, including 
free-of-charge health care. On the other hand, the absence of this effect in Bulgaria and Hungary might be related to the fact that people in these countries have already had experience with official co-payments and found that it turned out not to be harmful. The observed demand differences across place of residence which are observed in Hungary, but also in Bulgaria, Lithuania, and Poland are in line with the theoretical and empirical literature suggesting price discrimination by geographical area (McPake, 1993). However, the level and direction of this effect is to be cautiously approached as higher values expressed by dwellers of small cities in Bulgaria, Hungary, and Lithuania might actually be related to the relatively more difficult access to these services than in urban areas. This is the case in most countries despite the level of development (World Health Organization, 2008). Other sociodemographic factors have differential effects on the potential demand and their explanation requires a more detailed analysis of the systems features, which is out of scope of this study.

In this study we have shown how the stated WATP can be applied to the analysis of user charges in the context of a lack of actual experience with user charges. We do not provide a tool for the choice of the optimal fee level and we do not touch other normative questions regarding patient payments. There are concerns that together with informal payments they might impose a double burden on consumers and impair equity (Lewis, 2000). On the other hand, official charges together with anticorruption measures prove to be rather efficient in suppressing informal practices (Golinowska and Sowa, 2006; Lewis, 2006). We believe that an official patient payment policy that accounts for population WATP is likely to reduce the burden and inequality imposed by informal practices.

Although SP are argued to be a sound and valid source of information for price decisions in primary health care (Foreit and Fleischman Foreit, 2003; Mataria et al., 2007), they may be subject to hypothetical bias, i.e. may significantly overstate the W(A)TP derived from real market choices (Liljas and Blumenschein, 2000; Ryan and Watson, 2009). Statistical calibration techniques, like stochastic frontier models (e.g. Harder et al., 2006; Hofler and List, 2004), have yielded promising results in environmental and agricultural economics. Incorporating similar methods into the survival analysis of W(A)TP data on health care services might provide better estimates of the potential effects of user fees.

It should be also mentioned that the generic approach with semi-parametric estimations to cross-country data is subject to scale problems. For example, in our data many respondents drop out from the demand pool in the very first fee interval in Ukraine, but a rather small share does so in Poland. At the same time, in the payment interval from $€ 10.0$ to $€ 12.5$ the 


\section{Chapter 5}

situation is the opposite. This means that for the sake of policy-making, it would be more efficient to analyze in more detail the lower payment intervals in lower income countries, leaving out the higher ones, and doing the opposite for higher income countries. The payment cards should be presented to the respondents accordingly. This calls for additional singlecountry studies using country-specific measurement scales. 


\section{CHAPTER 6}

Calibration of the willingness to pay for physician services in six Central and Eastern European countries:

Context implications for the over- and understatement patterns

Submitted for publication 


\section{Chapter 6}

\section{Abstract}

Background: WTP/WATP obtained from SP techniques may be subject to hypothetical or strategic bias. This may lead to either over- or understatement of the true WTP/WATP.

Objective: In this study, we applied a stochastic frontier model to data on WATP for physician services in six CEE countries in order to test for strategic responses to WATP questions.

Methods: The CV data were collected in nationally representative surveys in Bulgaria, Hungary, Lithuania, Poland, Romania, and Ukraine in 2010.

Results: Understatement appears to be present in Bulgaria, where official co-payments are in effect. In the four countries, where co-payments for physician services have never been implemented, the general pattern suggests overstatement. Finally, in Hungary, where official co-payments existed for one year but were abolished afterwards, there is a slight overstatement but this is not statistically significant.

Conclusions: These findings suggest that over- or understatement may be related to the probability of a price changes as a consequence of the study. 


\subsection{Introduction}

$\mathrm{CV}$ is an increasingly used SP technique for the valuation of health care benefits (Smith and Sach, 2010; Klose, 1999; Diener et al., 1998). In a CV study, a specific health state or health care intervention is valued by an individual in terms of the stated WTP/WATP for that health state or intervention within a hypothetical scenario. Stated preference data have some important advantages over actual behavior data. Firstly, SP data can indicate the value that consumers attach to non-market goods, which is the case for most public goods. This is also the case for health care services that are either covered by collective health insurance policies or are publicly funded. Hence, the users of such services either meet no charges or pay charges that are lower than actual services costs. Additionally, SP data capture information about the value consumers attach to a market good, which can be different than the price that they pay for it. It also provides information about the value non-users attach to the use or the availability of goods, which cannot be deduced from actual behavior data (i.e. from information on the price users pay for the good).

Nevertheless, the results of CV are rarely applied to policy decisions about patient charges in health care. This is, most probably, because of the uncertain predictive validity of the stated WATP measures stemming from the hypothetical nature of the valuation tasks as discussed in Chapter 1. That is, WATP derived from a CV task is subject to a hypothetical bias, i.e. respondents may state WATP which is different from their true WATP if a real commitment is to be made (Loomis, 2011; Harrison and Rutström, 2008). Most empirical literature, largely from environmental economics, shows that CV produces overstated estimates of the actual WATP (Harrison and Rutström, 2008; Loomis, 2011; List and Gallet, 2001; Murphy et al., 2005). However, there are also indications that people might understate their real WATP (e.g. Walraven, 1996).

Both effects described above are based on theoretical mechanisms which basically arise from incentive incompatibility and, consequently, strategic behavior of respondents. On the one hand, if a respondent believes that the actual price will be based upon the stated WATP, this may create an incentive to understate their true WATP (Lunander, 1998; Samuelson, 1954). On the other hand, if respondents think that the higher stated WATP helps to ensure public provision of the good (i.e. the stated WATP is used to stress the importance that individual attach to a good or service), they may have an incentive to overstate their WATP. Hoehn and Randall (1987) argue that in case respondents believe that the information they provide will 


\section{Chapter 6}

influence policy decisions overstatement is likely to occur, while if it requires real contributions understatement is more likely. In both cases, it is expected that respondents believe that the survey results might influence real policies, either the policy of the public provision of the good or the policy of the pricing of the good. The causes and mechanisms of the hypothetical bias are not sufficiently studied yet (Mitani and Flores, 2010).

The approaches to deal with hypothetical bias can be distinguished into ex-ante methods, i.e. increasing the realism of the CV tasks, and ex-post techniques, where econometric techniques are used to correct the gathered data (Loomis, 2011). The latter methods in their early attempts implied trivial scaling of stated WATP by a certain ratio. The magnitude of the ratio was usually based upon empirical studies that compared the stated WATP to experimental or actual behavior (Fox et al, 1998). However, these studies did not provide conclusive evidence. For example, a meta-analysis by List and Gallet (2001) suggests a mean calibration ratio of 3, while the one by Murphy et al. (2005) suggests 2.6. In both reviews the range of the calibration ratios is very high: from less than 1 to more than 10 . Moreover, it is not likely that the estimates of these ratios are transferrable across studies and across interventions. Another approach to the ex-post correction for selection bias in $\mathrm{CV}$ studies is to estimate a bias function using socio-demographic characteristics (Blackburn et al., 1994; Mansfiled 1998). However, this only corrects for differences in stated WATP across individuals with different characteristics and does not solve the incentivizing problem. Studies that apply this approach also find that bias functions are not transferable across benefits.

An alternative approach to ex-post calibration is to apply a stochastic frontier analysis (SFA) to WATP data (Goldar and Misra, 2001; Hofler and List, 2004). The application of this calibration method to CV data has started relatively recently and until now there have been no application of SFA to correct for hypothetical bias in WATP estimates in the health economics field. In a SFA, the stated WATP is allowed to have an additional disturbance term with non-zero expectation. The linear predictor is assumed to represent the true WATP level, while the one-sided error term is thought to capture any potential bias. The advantage of this method is that it does not require additional data, only those gathered in the CV survey itself. The methodological assumption is about the direction of the additional error term: it is either positive if people generally overstate or negative if they generally understate their true WATP. It is a convention to assume overstatement in most applications; however, as discussed above, there are no grounds not to test for potential understatement as well. 
In this chapter, we apply a stochastic frontier model to data on WATP for physician services in six CEE countries in order to test for strategic responses to WATP questions. We relate the observed over- and understatement patterns derived from the estimates of the one-sided error terms to the country context in order to give an indication about the transferability of the results. We also compare both raw and calibrated WATP to the actual expenditures by respondents in the year prior to the CV study.

Our analysis is based on CV data collected in a national-representative survey in July-August 2010 in Bulgaria, Hungary, Lithuania, Poland, Romania, and Ukraine (as in the previous chapter). These countries present a relevant context for a cross-country comparison given the past similarities in their health care sectors during the communist period, as well as the different pace of health care reforms during the post-communist era. Moreover, Bulgaria is the only country in the study where patients are exposed to formal patient copayments for physician visits (1\% of minimum salary in the country per visit), and Hungary is the country with a one year experience of formal patient copayments for physician services (copayments were abolished in 2008). Therefore, this country set provides the possibility to explore hypothetical bias patterns not only in relation to socio-economic context, but also in relation to the naturalism of the monetary commitments.

\subsection{Methods}

We used the same data as in Chapter 5, i.e. from national representative household surveys held in July-August 2010 in the six CEE countries: Bulgaria, Hungary, Lithuania, Poland, Romania, and Ukraine. The target samples consisted of about 1000 respondents in each country. The sampling strategy is discussed in detail in Appendix B. For more details about the questionnaire see the previous chapter and Appendix D for the exact wording of the CV task. WATP was elicited via payment intervals followed by the exact statement within the selected interval.

As discussed above, despite the extensive empirical evidence that in $\mathrm{CV}$, respondents tend to overstate WATP, in situations where respondents perceive that fees might apply to them, WATP might be understated (Hoehn and Randall, 1987; Foster et al., 1997). In this study, both assumptions were tested. For this purpose, two stochastic frontier estimations were applied to WATP data: one with an additional half-normal disturbance term with a positive expectation, another with similar disturbance but a negative expectation. 


\section{Chapter 6}

The empirical stochastic frontier model is an extension of a linear model. It has the form of:

$$
\ln \left(W A T P_{i}\right)=\sum \beta X+\varepsilon_{i} \pm u_{i}
$$

where $\exp \left(\sum \beta X+\varepsilon_{i}\right)$ was assumed to be an expression of the true underlying WATP with stochastic error term $\varepsilon_{i} \sim N\left(0 ; \sigma_{\varepsilon}\right)$. Each observation may have an additional systematic deviation with expectation of $\pm u_{i}$, and $\exp \left( \pm u_{i}\right)$ is an inefficiency scale parameter, or the level of over- or understatement respectively (depending on the chosen sign). We assumed a half-normal distribution for systematic deviation, such that $\pm u_{i} \sim\left|N\left(0 ; \sigma_{u}\right)\right|$.

Both technically and theoretically, only one assumption about either a positive or a negative inefficiency term may hold. We applied a simple test (Hofler and List, 2004) for the skewness of residuals from the linear predictor to adopt the correct assumption. Hence, for each country only one model which corresponded to the appropriate skewness direction was selected. Based on this model, inefficiency scores were predicted for each respondent: $\left(\exp \left(+u_{i}\right)\right)$ for over- and $\left(\exp \left(-u_{i}\right)\right)$ for understatement models. Then, the stated individual WATP was

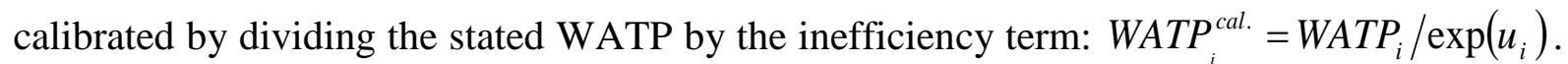
Thus, the stated WATP was scaled up in case of understated WATP or down in case of overstated WATP.

In case of a half-normal distribution, the zero expectation of the disturbance does not lead to a zero mean of the individual disturbances, as the whole density of the distribution is situated on either side of zero. In this case, another parameter is of interest $\sigma_{u}$ as it actually represents the thickness of the one tail of the systematic disturbance. Thus, a higher $\sigma_{u}$ actually represents a larger extent of under- or overstatement and, hence, leads to a higher mean disturbance and higher mean inefficiency scale $\overline{\exp \left( \pm u_{i}\right)}$.

Eventually, we compared both raw and scaled WATP measures to actual expenditure on physician services. This was done to see if the calibration improved the consistency of WATP with actual expenditure levels. However, WATP were measured per visit of a physician of high quality and with quick access, while the actual expenditure was for physician services of common quality and was measured on an annual basis. Therefore, due to these differences in measurement bases, we calculated predicted expenditure by multiplying the individuals' number of visits by his/her raw and calibrated WATP, i.e. what one would have spent if one had paid one's own WATP for each visit. Then we compared the aggregate means of the 
predicted and actual expenditures qualitatively, but also on an individual level quantitatively. The latter was done by means of Spearman rank correlation of the predicted expenditure to actual total (formal and informal expenditure, the latter being a characteristic feature of patient payments in the CEE countries included in the analysis). We checked for correlations with informal expenditures as we expected the informal payments to depend more on WATP of the consumers than the formal one.

\subsection{Results}

Descriptive characteristics of the samples are presented in Table B.2 in Appendix B. The samples well represent the adult population in the countries under study according to their national statistics. Country ranking by both household income and income per person is as follows: Polish respondents are the richest, followed by Hungarian, and then by the Lithuanian respondents. Respondents from the other three countries have substantially lower income in the following descending order: Romania, Bulgaria, and Ukraine. This ranking well represents the country ranking by macro-indicators of GNI per capita (The World Bank, 2013), except for the fact that Hungary has a slightly higher macro-indicator than Poland. The Polish sample is the youngest (average age: 44.12 years), slightly older are the Hungarian and Lithuanian samples on average (circa 46 years), then the Romanian and Ukrainian samples (circa 48 years), and the average age in the Bulgarian sample is the highest (50.4 years). Other sample characteristics are presented for reference as well (see Table B.2 in Appendix B).

Table B.2 (Appendix B) also presents the past experiences with physician visits and payments for them, as well as the patterns of WATP for the services of medical specialists of good quality and quick access in case of major health problems. Ukrainian respondents report the lowest level of service consumption with an average of 1.98 visits to a medical specialist per year. The levels of service consumption in Romania (3.24 visits), Lithuania (3.67 visits) and Poland (3.78 visits) are rather similar. The highest consumption level is observed in Hungary (5.26 visits) followed by Bulgaria (4.48 visits).

As indicated in Table B.2 (Appendix B), the lowest share of those who are not willing to pay is observed in Romania and Lithuania, as well as in Bulgaria. In Poland and Ukraine around $26 \%$ respondents are not willing to pay, while the highest unwillingness is observed in Hungary - around 33\%. In Bulgaria and Romania, the unwillingness is mostly explained by the inability to pay, in Lithuania and Ukraine slightly less emphasis is on inability than on 


\section{Chapter 6}

objection to pay, while in Hungary and Poland both reasons (unable and object to pay) are nearly equally prevalent.

The mean WATP (positive values only) is the highest in Poland (€ 15.11 for a visit), followed by WATP in Hungary of $€ 13.91$ per visit. Lithuanians and Romanians express similar WATP for a visit to a medical specialist: $€ 11.95$ and $€ 11.16$ respectively. Bulgarian respondents on average are willing to pay $€ 8.79$, while Ukrainian respondents have the lowest WATP: $€ 6.77$ (see Table B.2 in Appendix B). It should be noted that WATP levels follow closely the country ranking by average income reported by the respondents discussed above. The only outstanding case is Romania where WATP is relatively high approaching the level of Lithuania while income is substantially lower and very close to that in Bulgaria.

The results of the stochastic frontier analysis are presented in Table 6.1 together with the OLS results for comparison. The check of the skewness of the residuals from the OLS estimation shows that in Bulgaria, the understatement pattern is likely to be prevalent (negative skewness), while in the other five countries the overstatement pattern is more likely (positive skewness). In Bulgaria, the model based on the understatement assumption provides a rather significant one-sided variance. In Hungary, the positive-sided (overstatement) disturbance is present, but the test of its variance against zero shows a rather high probability of type-1 error (p-value is 0.19). This basically means that the larger share of the stated WATP is concentrated symmetrically around the linear predictor of the true WATP, while the density of understatements is comparatively low. In other countries, i.e. Lithuania, Poland, Romania, and Ukraine, the variance of the positive-sided disturbance differs from zero.

The results of the stochastic frontier modeling in terms of the predictors are rather consistent with those from the OLS estimation. Only some coefficients change in their level of significance when the inefficiency term is added, and only if they are not highly significant. As expected, the main predictor for the WATP level is income with a positive coefficient. The coefficients of $\ln$ (income) represent the income elasticity of WATP, which is low (less than 1) in all countries. The lowest income elasticity is in Poland (0.115), then in Romania (0.145), Bulgaria (0.175), Lithuania (0.166), and it is the highest in Hungary (0.241). In Ukraine, WATP appears to be rather homogeneous among income groups. In Hungary, males are willing to pay on average $13 \%$ more than women. Place of residence also plays role in Bulgaria and Hungary. In Bulgaria, small city dwellers are willing to pay more per visit than the rest of the population, while in Hungary and Lithuania, big city dwellers have a lower WATP than the others. 
Table 6.1 Stochastic frontier estimation of the WATP level for physician services

\begin{tabular}{|c|c|c|c|c|c|c|c|}
\hline \multirow[b]{2}{*}{ Effect } & & \multicolumn{2}{|c|}{ Bulgaria } & \multicolumn{2}{|c|}{ Hungary } & \multicolumn{2}{|c|}{ Lithuania } \\
\hline & & OLS & $\begin{array}{l}\text { Frontier } \\
\text { (underst.) }\end{array}$ & OLS & $\begin{array}{l}\text { Frontier } \\
\text { (overst.) }\end{array}$ & OLS & $\begin{array}{l}\text { Frontier } \\
\text { (overst.) }\end{array}$ \\
\hline \multicolumn{2}{|l|}{$\overline{\text { Ln(age) }}$} & $\begin{array}{l}-0.024 \\
(0.082)\end{array}$ & $\begin{array}{l}-0.025 \\
(0.080)\end{array}$ & $\begin{array}{l}-0.012 \\
(0.081)\end{array}$ & $\begin{array}{l}-0.027 \\
(0.076)\end{array}$ & $\begin{array}{l}-0.089 \\
(0.078)\end{array}$ & $\begin{array}{l}-0.09 \\
(0.075)\end{array}$ \\
\hline \multicolumn{2}{|l|}{ Ln(income) } & $\begin{array}{l}0.175^{* * *} \\
(0.047)\end{array}$ & $\begin{array}{l}0.175^{* * * *} \\
(0.047)\end{array}$ & $\begin{array}{l}0.240 * * * \\
(0.069)\end{array}$ & $\begin{array}{l}0.241^{* * * *} \\
(0.065)\end{array}$ & $\begin{array}{l}0.174 * * * \\
(0.036)\end{array}$ & $\begin{array}{l}0.166 * * * \\
(0.036)\end{array}$ \\
\hline \multicolumn{2}{|c|}{ Ln(household size) } & $\begin{array}{l}-0.109 * \\
(0.060)\end{array}$ & $\begin{array}{l}-0.109 * \\
(0.058)\end{array}$ & $\begin{array}{l}-0.121 * * \\
(0.068)\end{array}$ & $\begin{array}{l}-0.087 \\
(0.065)\end{array}$ & $\begin{array}{l}-0.115 * \\
(0.055)\end{array}$ & $\begin{array}{l}-0.045 \\
(0.053)\end{array}$ \\
\hline Sex & $\begin{array}{l}\text { female } \\
\text { male }\end{array}$ & $\begin{array}{l}\text { ref. } \\
0.061 \\
(0.046) \\
\end{array}$ & $\begin{array}{l}\text { ref. } \\
0.066 \\
(0.045) \\
\end{array}$ & $\begin{array}{l}\text { ref. } \\
0.131^{* *} \\
(0.062) \\
\end{array}$ & $\begin{array}{l}\text { ref. } \\
0.133^{* *} \\
(0.059) \\
\end{array}$ & $\begin{array}{l}\text { ref. } \\
-0.031 \\
(0.049) \\
\end{array}$ & $\begin{array}{l}\text { ref. } \\
-0.033 \\
(0.047) \\
\end{array}$ \\
\hline \multirow[t]{2}{*}{ Residence } & $\begin{array}{l}\text { village } \\
\text { small city }\end{array}$ & $\begin{array}{l}\text { ref. } \\
0.234^{* * *} \\
(0.055)\end{array}$ & $\begin{array}{l}\text { ref. } \\
0.203^{* * *} \\
(0.055)\end{array}$ & $\begin{array}{l}\text { ref. } \\
0.085 \\
(0.071)\end{array}$ & $\begin{array}{l}\text { ref. } \\
0.057 \\
(0.067)\end{array}$ & $\begin{array}{l}\text { ref. } \\
-0.023 \\
(0.057)\end{array}$ & $\begin{array}{l}\text { ref. } \\
0.033 \\
(0.056)\end{array}$ \\
\hline & big city & $\begin{array}{l}0.062 \\
(0.069)\end{array}$ & $\begin{array}{l}0.034 \\
(0.068)\end{array}$ & $\begin{array}{l}-0.470^{* * *} \\
(0.097)\end{array}$ & $\begin{array}{l}-0.484^{* * *} \\
(0.093)\end{array}$ & $\begin{array}{l}-0.225^{* * *} \\
(0.067)\end{array}$ & $\begin{array}{l}-0.179 * * * \\
(0.066)\end{array}$ \\
\hline \multirow[t]{3}{*}{ Education } & \multirow{2}{*}{$\begin{array}{l}\text { no or primary } \\
\text { secondary }\end{array}$} & ref. & ref. & ref. & ref. & ref. & ref. \\
\hline & & $\begin{array}{l}0.065 \\
(0.067) \\
\end{array}$ & $\begin{array}{l}0.061 \\
(0.066) \\
\end{array}$ & $\begin{array}{l}0.116 \\
(0.072) \\
\end{array}$ & $\begin{array}{l}0.11 \\
(0.068)\end{array}$ & $\begin{array}{l}-0.002 \\
(0.083) \\
\end{array}$ & $\begin{array}{l}-0.018 \\
(0.080) \\
\end{array}$ \\
\hline & high+ & $\begin{array}{l}0.159 * \\
(0.081)\end{array}$ & $\begin{array}{l}0.136 * \\
(0.079)\end{array}$ & $\begin{array}{l}0.03 \\
(0.093)\end{array}$ & $\begin{array}{l}0.035 \\
(0.088) \\
\end{array}$ & $\begin{array}{l}0.024 \\
(0.084) \\
\end{array}$ & $\begin{array}{l}0.002 \\
(0.081) \\
\end{array}$ \\
\hline \multirow[t]{3}{*}{ Health status } & $\begin{array}{l}\text { very bad } \\
\text { fair }\end{array}$ & $\begin{array}{l}\text { ref. } \\
0.018 \\
(0.084) \\
\end{array}$ & $\begin{array}{l}\text { ref. } \\
-0.021 \\
(0.083) \\
\end{array}$ & $\begin{array}{l}\text { ref. } \\
0.077 \\
(0.108) \\
\end{array}$ & $\begin{array}{l}\text { ref. } \\
0.183^{*} \\
(0.103) \\
\end{array}$ & $\begin{array}{l}\text { ref. } \\
-0.054 \\
(0.091) \\
\end{array}$ & $\begin{array}{l}\text { ref. } \\
-0.012 \\
(0.089) \\
\end{array}$ \\
\hline & good & $\begin{array}{l}0.018 \\
(0.084)\end{array}$ & $\begin{array}{l}-0.03 \\
(0.084)\end{array}$ & $\begin{array}{l}0.145 \\
(0.110)\end{array}$ & $\begin{array}{l}0.244^{* *} \\
(0.105)\end{array}$ & $\begin{array}{l}-0.012 \\
(0.098)\end{array}$ & $\begin{array}{l}0.019 \\
(0.096)\end{array}$ \\
\hline & perfect & $\begin{array}{l}0.065 \\
(0.092)\end{array}$ & $\begin{array}{l}-0.014 \\
(0.093) \\
\end{array}$ & $\begin{array}{l}0.136 \\
(0.118)\end{array}$ & $\begin{array}{l}0.236 * * \\
(0.113)\end{array}$ & $\begin{array}{l}-0.051 \\
(0.110) \\
\end{array}$ & $\begin{array}{l}0.009 \\
(0.109) \\
\end{array}$ \\
\hline \multicolumn{2}{|l|}{ Constant } & $\begin{array}{l}0.924^{* *} \\
(0.438) \\
\end{array}$ & $\begin{array}{l}1.479 * * * \\
(0.450)\end{array}$ & $\begin{array}{l}0.702 \\
(0.497) \\
\end{array}$ & $\begin{array}{l}-0.01 \\
(0.467)\end{array}$ & $\begin{array}{l}1.639 * * * \\
(0.386) \\
\end{array}$ & $\begin{array}{l}1.050^{* * *} \\
(0.374)\end{array}$ \\
\hline \multicolumn{8}{|l|}{$\overline{L n}(\operatorname{sig} 2(\varepsilon))$} \\
\hline \multicolumn{2}{|l|}{ Constant } & & $\begin{array}{l}-1.570 * * * \\
(0.142)\end{array}$ & & $\begin{array}{l}-1.421^{* * *} \\
(0.148)\end{array}$ & & $\begin{array}{l}-1.503^{* * *} \\
(0.117)\end{array}$ \\
\hline \multicolumn{8}{|c|}{ Ln(sig2(u)): inefficiency } \\
\hline Service user & & & $\begin{array}{l}0.507 * \\
(0.267)\end{array}$ & & $\begin{array}{l}-0.062 \\
(0.198)\end{array}$ & & $\begin{array}{l}0.089 \\
(0.169)\end{array}$ \\
\hline Constant & & & $\begin{array}{l}-1.502^{* * *} \\
(0.390) \\
\end{array}$ & & $\begin{array}{l}-0.146 \\
(0.219)\end{array}$ & & $\begin{array}{l}-0.701^{* * *} \\
(0.198) \\
\end{array}$ \\
\hline \multicolumn{8}{|c|}{ Model statistics } \\
\hline \multirow{2}{*}{\multicolumn{2}{|c|}{$\begin{array}{l}\text { Number of observations } \\
\text { F-test } \\
\text { Likelihood ratio test (chi2) } \\
\mathrm{R}^{2}\end{array}$}} & $\begin{array}{l}661 \\
6.37 * * *\end{array}$ & 654 & $\begin{array}{l}616 \\
5.98 * * *\end{array}$ & 613 & $\begin{array}{l}777 \\
3.50 \text { *** }\end{array}$ & 777 \\
\hline & & 0.0974 & $52.79 * * *$ & 0.0983 & $72.32 * * *$ & 0.0479 & $40.41^{* * *}$ \\
\hline Adj. $R^{2}$ & & 0.0821 & & 0.0818 & & 0.0342 & \\
\hline Log-likelih & ood & -574.80 & -563.13 & -688.91 & -671.33 & -766.31 & -753.46 \\
\hline AIC & & 1173.61 & 1156.25 & 1401.8 & 1372.72 & 1556.61 & 1536.92 \\
\hline $\mathrm{BIC}$ & & 1227.53 & 1223.5 & 1454.9 & 1438.91 & 1612.48 & 1606.75 \\
\hline
\end{tabular}


Table 6.1 (continued) Stochastic frontier estimation of the WATP level for physician services

\begin{tabular}{|c|c|c|c|c|c|c|c|}
\hline \multirow[b]{2}{*}{ Effect } & & \multicolumn{2}{|c|}{ Poland } & \multicolumn{2}{|c|}{ Romania } & \multicolumn{2}{|c|}{ Ukraine } \\
\hline & & OLS & $\begin{array}{l}\text { Frontier } \\
\text { (overst.) }\end{array}$ & OLS & $\begin{array}{l}\text { Frontier } \\
\text { (overst.) }\end{array}$ & OLS & $\begin{array}{l}\text { Frontier } \\
\text { (overst.) }\end{array}$ \\
\hline \multicolumn{2}{|l|}{$\begin{array}{l}\text { Ln(age) } \\
\text { (age }\end{array}$} & $\begin{array}{l}-0.078 \\
(0.089)\end{array}$ & $\begin{array}{l}-0.098 \\
(0.087)\end{array}$ & $\begin{array}{l}-0.082 \\
(0.091)\end{array}$ & $\begin{array}{l}-0.07 \\
(0.087)\end{array}$ & $\begin{array}{l}-0.055 \\
(0.081)\end{array}$ & $\begin{array}{l}-0.053 \\
(0.079) \\
\end{array}$ \\
\hline \multicolumn{2}{|l|}{ Ln(income) } & $\begin{array}{l}0.120^{* *} \\
(0.058)\end{array}$ & $\begin{array}{l}0.115^{* *} \\
(0.057)\end{array}$ & $\begin{array}{l}0.136 * * * \\
(0.040)\end{array}$ & $\begin{array}{l}0.145^{* * *} \\
(0.041)\end{array}$ & $\begin{array}{l}-0.009 \\
(0.049)\end{array}$ & $\begin{array}{l}-0.019 \\
(0.049) \\
\end{array}$ \\
\hline \multicolumn{2}{|c|}{ Ln(household size) } & $\begin{array}{l}-0.031 \\
(0.064)\end{array}$ & $\begin{array}{c}-0.031 \\
(0.063)\end{array}$ & $\begin{array}{c}-0.052 \\
(0.059)\end{array}$ & $\begin{array}{c}-0.01 \\
(0.056)\end{array}$ & $\begin{array}{c}-0.053 \\
(0.062)\end{array}$ & $\begin{array}{c}0.094 \\
(0.061)\end{array}$ \\
\hline Sex & $\begin{array}{l}\text { female } \\
\text { male }\end{array}$ & $\begin{array}{l}\text { ref. } \\
0.066 \\
(0.055)\end{array}$ & $\begin{array}{l}\text { ref. } \\
0.048 \\
(0.054) \\
\end{array}$ & $\begin{array}{l}\text { ref. } \\
-0.069 \\
(0.055) \\
\end{array}$ & $\begin{array}{l}\text { ref. } \\
-0.103^{*} \\
(0.053)\end{array}$ & $\begin{array}{l}\text { ref. } \\
-0.013 \\
(0.055) \\
\end{array}$ & $\begin{array}{l}\text { ref. } \\
-0.007 \\
(0.054) \\
\end{array}$ \\
\hline \multirow[t]{2}{*}{ Residence } & $\begin{array}{l}\text { village } \\
\text { small city }\end{array}$ & $\begin{array}{l}\text { ref. } \\
-0.115^{*} \\
(0.063)\end{array}$ & $\begin{array}{l}\text { ref. } \\
-0.124^{* *} \\
(0.062) \\
\end{array}$ & $\begin{array}{l}\text { ref. } \\
-0.017 \\
(0.062)\end{array}$ & $\begin{array}{l}\text { ref. } \\
-0.052 \\
(0.058)\end{array}$ & $\begin{array}{l}\text { ref. } \\
-0.03 \\
(0.066)\end{array}$ & $\begin{array}{l}\text { ref. } \\
-0.042 \\
(0.065)\end{array}$ \\
\hline & big city & $\begin{array}{l}0.114 \\
(0.075)\end{array}$ & $\begin{array}{l}0.101 \\
(0.073) \\
\end{array}$ & $\begin{array}{l}0.028 \\
(0.077) \\
\end{array}$ & $\begin{array}{l}0.032 \\
(0.073) \\
\end{array}$ & $\begin{array}{l}0.042 \\
(0.067) \\
\end{array}$ & $\begin{array}{l}0.034 \\
(0.066)\end{array}$ \\
\hline \multirow[t]{2}{*}{ Education } & $\begin{array}{l}\text { no or primary } \\
\text { secondary }\end{array}$ & $\begin{array}{l}\text { ref. } \\
-0.142^{* *} \\
(0.072)\end{array}$ & $\begin{array}{l}\text { ref. } \\
-0.120^{*} \\
(0.070)\end{array}$ & $\begin{array}{l}\text { ref. } \\
0.013 \\
(0.079)\end{array}$ & $\begin{array}{l}\text { ref. } \\
0.028 \\
(0.075)\end{array}$ & $\begin{array}{l}\text { ref. } \\
0.144 \\
(0.128)\end{array}$ & $\begin{array}{l}\text { ref. } \\
0.137 \\
(0.126)\end{array}$ \\
\hline & high+ & $\begin{array}{l}-0.134 \\
(0.096) \\
\end{array}$ & $\begin{array}{l}-0.103 \\
(0.094) \\
\end{array}$ & $\begin{array}{l}0.067 \\
(0.093) \\
\end{array}$ & $\begin{array}{l}0.12 \\
(0.089) \\
\end{array}$ & $\begin{array}{l}0.172 \\
(0.134) \\
\end{array}$ & $\begin{array}{l}0.175 \\
(0.132) \\
\end{array}$ \\
\hline \multirow[t]{3}{*}{ Health status } & $\begin{array}{l}\text { very bad } \\
\text { fair }\end{array}$ & $\begin{array}{l}\text { ref. } \\
-0.062 \\
(0.102)\end{array}$ & $\begin{array}{l}\text { ref. } \\
-0.085 \\
(0.099)\end{array}$ & $\begin{array}{l}\text { ref. } \\
-0.024 \\
(0.089)\end{array}$ & $\begin{array}{l}\text { ref. } \\
0.016 \\
(0.082)\end{array}$ & $\begin{array}{l}\text { ref. } \\
-0.046 \\
(0.073)\end{array}$ & $\begin{array}{l}\text { ref. } \\
-0.029 \\
(0.074)\end{array}$ \\
\hline & good & $\begin{array}{l}0.04 \\
(0.105)\end{array}$ & $\begin{array}{l}0.023 \\
(0.101)\end{array}$ & $\begin{array}{l}0.026 \\
(0.092)\end{array}$ & $\begin{array}{l}0.049 \\
(0.085)\end{array}$ & $\begin{array}{l}0.031 \\
(0.093)\end{array}$ & $\begin{array}{l}0.055 \\
(0.095)\end{array}$ \\
\hline & perfect & $\begin{array}{l}0.017 \\
(0.117)\end{array}$ & $\begin{array}{l}-0.015 \\
(0.115)\end{array}$ & $\begin{array}{l}-0.026 \\
(0.117)\end{array}$ & $\begin{array}{l}-0.018 \\
(0.110)\end{array}$ & $\begin{array}{l}0.107 \\
(0.133)\end{array}$ & $\begin{array}{l}0.142 \\
(0.132)\end{array}$ \\
\hline \multicolumn{2}{|l|}{ Constant } & $\begin{array}{l}2.105^{* * * *} \\
(0.492) \\
\end{array}$ & $\begin{array}{l}1.741^{* * * *} \\
(0.481)\end{array}$ & $\begin{array}{l}1.668 * * * \\
(0.422) \\
\end{array}$ & $\begin{array}{l}0.919 * * \\
(0.410)\end{array}$ & $\begin{array}{l}1.684^{* * *} \\
(0.429)\end{array}$ & $\begin{array}{l}1.232 * * * \\
(0.427)\end{array}$ \\
\hline \multicolumn{2}{|l|}{ Constant } & & $\begin{array}{l}-1.294^{* * *} \\
(0.132)\end{array}$ & & $\begin{array}{l}-1.593^{* * *} \\
(0.136)\end{array}$ & & $\begin{array}{l}-1.224^{* * *} \\
(0.142)\end{array}$ \\
\hline \multicolumn{2}{|c|}{ Service user } & & $\begin{array}{l}-0.197 \\
(0.227)\end{array}$ & & $\begin{array}{l}-0.319 * * \\
(0.150)\end{array}$ & & $\begin{array}{l}0.092 \\
(0.202)\end{array}$ \\
\hline \multicolumn{2}{|l|}{ Constant } & & $\begin{array}{l}-0.732^{* *} \\
(0.298) \\
\end{array}$ & & $\begin{array}{l}-0.135 \\
(0.157) \\
\end{array}$ & & $\begin{array}{l}-1.034^{* * *} \\
(0.335)\end{array}$ \\
\hline \multicolumn{8}{|c|}{ Model statistics } \\
\hline \multirow{2}{*}{\multicolumn{2}{|c|}{$\begin{array}{l}\text { Number of observations } \\
\text { F-test } \\
\text { Wald test (chi2) }\end{array}$}} & $\begin{array}{l}614 \\
2.52^{* * *}\end{array}$ & 614 & $\begin{array}{l}694 \\
2.49^{* * *}\end{array}$ & 692 & $\begin{array}{l}660 \\
1.15\end{array}$ & 658 \\
\hline & & & $27.72 * * *$ & & $37.48 * * *$ & & 13.90 \\
\hline $\begin{array}{l}\mathrm{R}^{2} \\
\text { Adj. } \mathrm{R}^{2}\end{array}$ & & $\begin{array}{l}0.0440 \\
0.0266\end{array}$ & & $\begin{array}{l}0.0387 \\
0.0231\end{array}$ & & $\begin{array}{l}0.0191 \\
0.0025\end{array}$ & \\
\hline Log-likelih & & -610.37 & -605.84 & -725.15 & -702.63 & -660.05 & -654.35 \\
\hline AIC & & 1244.74 & 1241.68 & 1474.3 & 1435.25 & 1344.10 & 1338.71 \\
\hline BIC & & 1297.78 & 1307.98 & 1528.81 & 1503.35 & 1398.01 & 1406.04 \\
\hline
\end{tabular}


Other characteristics have some but not a very strong effect. In Ukraine, WATP is practically not related to any of the explanatory characteristics, i.e. is homogeneous across population groups. Parameterization of the inefficiency term (under- or overstatement) shows that in Bulgaria users of services are more likely to understate their WATP, while in Romania they are less likely to overstate it. In other four countries, the inefficiency term is not related to the health care use or payment experience.

The average ratios of over- or understatement are presented in Table 6.2. Among Bulgarian respondents the average understatement ratio is 0.67 . In the other five countries, respondents tend to overstate with the mean overstatement ranging from 1.7 to 2.5. The difference in mean inefficiency scores is significant (by Scheffe comparisons) except for those in Romania and Hungary, and in Ukraine and Poland. Table 6.2 also contains the estimates for the mean WATP on the raw scale and mean calibrated WATP which account for the over- and understatement.

Table 6.2 Calibrated WTP for physician services using inefficiency scores

\begin{tabular}{|c|c|c|c|c|c|c|c|c|c|c|}
\hline \multirow{3}{*}{ Country } & \multirow{3}{*}{$\begin{array}{c}\text { Mean } \\
\text { ineffici } \\
\text { ency } \\
\text { score }\end{array}$} & \multirow{2}{*}{\multicolumn{2}{|c|}{$\begin{array}{c}\text { Mean WATP } \\
\text { (positive), } €\end{array}$}} & \multirow{2}{*}{\multicolumn{2}{|c|}{$\begin{array}{c}\text { Average predicted } \\
\text { expenditure } \\
\left.\text { (WATP }_{\mathrm{i}}^{*} \text { visits }_{\mathrm{i}}\right), €\end{array}$}} & \multirow{3}{*}{$\begin{array}{l}\text { Average } \\
\text { actual } \\
\text { expendit } \\
\text { ure, } €\end{array}$} & \multicolumn{4}{|c|}{ Spearman rank correlation } \\
\hline & & & & & & & \multicolumn{2}{|c|}{$\begin{array}{c}\text { Predicted and } \\
\text { total actual } \\
\text { expenditure }\end{array}$} & \multicolumn{2}{|c|}{$\begin{array}{l}\text { Predicted and } \\
\text { informal } \\
\text { actual } \\
\text { expenditure }\end{array}$} \\
\hline & & $\begin{array}{l}\text { WATP } \\
\text { raw }\end{array}$ & $\begin{array}{l}\text { WATP } \\
\text { scaled }\end{array}$ & $\begin{array}{c}\text { WATP } \\
\text { raw }\end{array}$ & $\begin{array}{l}\text { WATP } \\
\text { scaled }\end{array}$ & & $\begin{array}{l}\text { WATP } \\
\text { raw }\end{array}$ & $\begin{array}{l}\text { WATP } \\
\text { scaled }\end{array}$ & $\begin{array}{c}\text { WATP } \\
\text { raw }\end{array}$ & $\begin{array}{l}\text { WATP } \\
\text { scaled } \\
\end{array}$ \\
\hline Bulgaria & $\begin{array}{c}0.67 \\
(0.00)\end{array}$ & $\begin{array}{c}8.79 \\
(0.25)\end{array}$ & $\begin{array}{l}12.38 \\
(0.30)\end{array}$ & $\begin{array}{l}35.84 \\
(3.79)\end{array}$ & $\begin{array}{l}52.62 \\
(4.85)\end{array}$ & $\begin{array}{l}16.73 \\
(2.48)\end{array}$ & $\begin{array}{c}0.55 \\
(0.00)\end{array}$ & $\begin{array}{c}0.55 \\
(0.00)\end{array}$ & $\begin{array}{c}0.21 \\
(0.00)\end{array}$ & $\begin{array}{c}0.21 \\
(0.00)\end{array}$ \\
\hline Hungary & $\begin{array}{c}2.50 \\
(0.09)\end{array}$ & $\begin{array}{l}13.91 \\
(0.83)\end{array}$ & $\begin{array}{c}4.76 \\
(0.07)\end{array}$ & $\begin{array}{l}81.61 \\
(9.89)\end{array}$ & $\begin{array}{l}25.81 \\
(1.23)\end{array}$ & $\begin{array}{l}16.83 \\
(1.91)\end{array}$ & $\begin{array}{c}0.45 \\
(0.00)\end{array}$ & $\begin{array}{c}0.44 \\
(0.00)\end{array}$ & $\begin{array}{c}0.41 \\
(0.00)\end{array}$ & $\begin{array}{c}0.40 \\
(0.00)\end{array}$ \\
\hline Lithuania & $\begin{array}{c}2.01 \\
(0.04)\end{array}$ & $\begin{array}{l}11.95 \\
(0.50)\end{array}$ & $\begin{array}{c}5.23 \\
(0.07)\end{array}$ & $\begin{array}{l}43.65 \\
(5.29)\end{array}$ & $\begin{array}{l}\mathbf{1 7 . 8 7} \\
(1.02)\end{array}$ & $\begin{array}{l}\mathbf{2 6 . 9 8} \\
(3.09)\end{array}$ & $\begin{array}{c}0.49 \\
(0.00)\end{array}$ & $\begin{array}{c}0.50 \\
(0.00)\end{array}$ & $\begin{array}{c}0.33 \\
(0.00)\end{array}$ & $\begin{array}{c}0.34 \\
(0.00)\end{array}$ \\
\hline Poland & $\begin{array}{c}1.82 \\
(0.03)\end{array}$ & $\begin{array}{l}15.11 \\
(0.54)\end{array}$ & $\begin{array}{c}7.41 \\
(0.13)\end{array}$ & $\begin{array}{l}58.65 \\
(6.62)\end{array}$ & $\begin{array}{l}28.91 \\
(1.92)\end{array}$ & $\begin{array}{l}14.36 \\
(1.56)\end{array}$ & $\begin{array}{c}0.43 \\
(0.00)\end{array}$ & $\begin{array}{c}0.43 \\
(0.00)\end{array}$ & $\begin{array}{c}0.25 \\
(0.00)\end{array}$ & $\begin{array}{c}0.26 \\
(0.00)\end{array}$ \\
\hline Romania & $\begin{array}{c}2.31 \\
(0.06)\end{array}$ & $\begin{array}{l}11.16 \\
(0.50)\end{array}$ & $\begin{array}{c}4.25 \\
(0.05)\end{array}$ & $\begin{array}{l}34.59 \\
(3.55)\end{array}$ & $\begin{array}{l}13.77 \\
(0.79)\end{array}$ & $\begin{array}{l}\mathbf{4 8 . 9 2} \\
(8.76)\end{array}$ & $\begin{array}{c}0.52 \\
(0.00)\end{array}$ & $\begin{array}{c}0.53 \\
(0.00)\end{array}$ & $\begin{array}{c}0.37 \\
(0.00)\end{array}$ & $\begin{array}{c}0.38 \\
(0.00)\end{array}$ \\
\hline Ukraine & $\begin{array}{c}1.76 \\
(0.02)\end{array}$ & $\begin{array}{c}6.77 \\
(0.29)\end{array}$ & $\begin{array}{c}3.34 \\
(0.06)\end{array}$ & $\begin{array}{l}15.13 \\
(3.77)\end{array}$ & $\begin{array}{c}\mathbf{6 . 4 2} \\
(0.82)\end{array}$ & $\begin{array}{l}19.92 \\
(2.43)\end{array}$ & $\begin{array}{c}0.61 \\
(0.00)\end{array}$ & $\begin{array}{c}0.62 \\
(0.00)\end{array}$ & $\begin{array}{c}0.47 \\
(0.00)\end{array}$ & $\begin{array}{c}0.48 \\
(0.00)\end{array}$ \\
\hline
\end{tabular}

Comparison of WATP with the actual expenditures on physician services is also presented in Table 6.2. In case of Hungary, Lithuania, and Poland, the calibration makes the mean predicted expenditure more similar to actual expenditure, while in Bulgaria, Romania, and Ukraine it sets them more apart. At the individual level, we also checked for improvements of the correlation (Spearman rank) between predicted and actual total and informal expenditure. 


\section{Chapter 6}

Again, calibration does not lead to improvement in the consistency between them. Thus, these comparisons do not provide evidence for the validation of either of the WATP measures: neither raw, nor calibrated.

\subsection{Discussion and conclusion}

WATP calibration in the policy-making context is typically used to obtain more conservative estimates (Arrow and Solow, 1993). From this perspective, substantiation of the understatement model is not desirable as it only inflates the results. In such framework, we should have disregarded the understatement hypotheses, and the model for Bulgaria would simply resort to OLS estimates and no to the WATP calibration. However, we do not see grounds to disregard the possibility of understatement.

The models appear to satisfy theoretical expectations. Income is the strongest positive predictor of the WATP level in both OLS and SFA in five countries, except Ukraine. In addition to income, age and place of residence are also recognized as the main WATP drivers in the literature, and are therefore proposed as key price discrimination criteria (McPake, 1993). We, however, do not find any difference across age, but place of residence does play a role in Bulgaria (higher WATP in small city dwellers than in rural areas), Poland (lower WATP in small city dwellers than in rural area), and in Hungary and Lithuania (higher WATP in rural areas than in big city dwellers). Ukraine is a special case as none of the predictors is significant. We explain this by a well-established practice of informal charges (Gryga et al., 2010), which shapes the perception of commonly accepted payment levels and, consequently, the stated WATP levels.

In our study, where the same survey instrument was applied to the same physician services in different countries, a great variety is observed, including qualitative differences (overstatement and understatement). Thus our results demonstrate that the hypothetical bias correction mechanisms are context specific and, most probably, are not transferrable across countries. The range of the country average observed calibration ratios falls between 0.67 and 2.5. Within the countries these ratios also have a big range, though their overall variation is small. The great variety of calibration ratios is in line with the earlier studies (for reviews see List and Gallet, 2001; Murphy et al., 2005). The mentioned reviews suggest that on average the calibration (overstatement) ratios are in the order between 2 and 3 which is comparable to what we observe. 
We lack evidence to explain the quantitative differences at a cross-national level (i.e. the magnitude of the mean bias); however, we find that qualitative difference might be related to the realism of price changes as a consequence of the study. This aspect is strongest in Bulgaria, where patients do currently pay for physician services. Moreover, in the beginning of 2010, before the survey was held, there were discussions about a new law on mandatory health insurance which could bring possible new patient changes (Radu, 2010). Simultaneously, in this country we observe the significant left skewness of WATP data towards smaller values, and, consequently, the understatement assumption is supported.

In the four countries which have no experience with official patient charges for physician services in the public sector (Lithuania, Poland, Romania) or they are even illegal (Ukraine), right skewness, i.e. overstatement, is found to be significant. In Hungary, WATP is somewhat skewed to the right which results in an insignificant average overstatement of WATP. We again explain the tendency to overstate by the low realism of the consequential price arrangements, as the official copayments were rejected during a national population referendum in Hungary (Edelényi et al., 2008). The fact that this overstatement is not significant in Hungary might be related to the fact that in Hungary, people have already had experience with the official fee implementation, which makes the realism of the potential price arrangements higher as opposed to other four countries. On the other hand, since the reintroduction of these charges is not an issue in Hungary (neither during the survey nor at the moment), Hungarians have no specific reason to understate their WATP.

Additionally, we find within country support for the idea that understatement is related to the perceived risk of the payment policy changes. This is the association of higher understatement levels in Bulgaria and lower overstatement in Romania with the higher service use. For a service user, in case of fee implementation, the real monetary commitments are more likely to occur than for a non-user. Hence, the perceived risks should be higher. Consequently, users are expected to understate their WATP.

If the assumptions of the stochastic frontier model hold, the evidence described above brings together theories about the nature of the strategic behavior (Hoehn and Randall, 1987; Carson and Groves, 2007). Thus, the strategic answering patterns might be seen as the result of a trade-off a respondent makes between the desirability of the introduction of quality gains and the likelihood of pricing decisions as a result of the survey. In the countries where copayments are not introduced or limited, their introduction is an unlikely political action. Hence, respondents, who believe that the likelihood that their responses have consequences 


\section{Chapter 6}

for pricing policies is low but the desire for quality improvements is high, might tend to overstate their true WATP. In countries where co-payments exist, the likelihood of the consequential pricing arrangements is higher. This might prevent respondents from overstating their WATP and provoke concealing their real WATP.

Our results provide empirical confirmation of the arguments of institutional economists and economic sociologists that economic action is not fully rational but it is embedded in the system of social relations (Granovetter, 1985) and, hence, is affected by institutional arrangements. Such findings bring the idea that there is much more heterogeneity in stated WATP than that which could be explained by observed factors, and part of it is related to the perceived risk of subsequent payment changes. One of the strategies to address this, applied in the present chapter, is to assume that there is a portion of 'true' WATP answers and try to retrieve it by allowing over- and/or understatement patterns as measured by the one-sided error term in the stochastic frontier analysis.

In reality, assuming that respondents might have only one direction of strategic answering might be too restrictive. A possible solution is to search for 'true WATP' in the middle of the data, that is, allow simultaneous over- and understatement residuals in one model. This is possible by the application of the two-tiered stochastic frontier (Polachek and Yoon, 1996, 1987; Groot and van den Brink, 2007; Hofler and List, 2004; Tomini et al., 2012a). Up till recently, the two-tiered estimator was limited to the case of two exponentially distributed inefficiency terms (Polachek and Yoon, 1996), which is restrictive and had convergence problems in application to our data. More flexible distributions of inefficiency terms (e.g. Tsionas, 2012) might make two-tiered stochastic frontier estimation more applicable to WATP data. Additionally, it is possible that there are several groups with their own "true WATP' and, respectively, their own strategic/hypothetical bias pattern. In our data we observe several peaks which might indicate this. In this case, the relatively new latent class stochastic models (finite mixture models) are a good solution for accommodating this heterogeneity (Orea and Kumbhakar, 2004; Greene, 2005). 
CHAPTER 7

General discussion 


\subsection{Introduction}

Generally, there is a lack of evidence from CEE countries about the effects of official patient charges on health care consumption due to limited experience with such payments. In this situation, as outlined in Chapter 1, SP techniques are a useful tool to explore these effects and to provide a base for informed decisions about the patient payment policies in a country. However, SP techniques, due to their hypothetical nature, are rarely trusted as a reliable source of information about the true preferences and WTP, especially for policy decisions.

While empirical studies are needed to demonstrate the level of applicability of SP techniques in policy analysis, this research area remains rather underexplored. Therefore, this dissertation is focused on the application of SP techniques to the assessment of patient payments, specifically patient payments for basic physician services in CEE countries. The dissertation aim is formulated as follows: to apply SP techniques to study WTP for physician services in six CEE countries (Bulgaria, Hungary, Lithuania, Poland, Romania, and Ukraine), and to analyze to what extent WTP estimates can be used in the assessment of patient charges for physician services.

To achieve this aim, the dissertation presents several empirical analyses. The analyses are based on two datasets: first, from a small-scale survey in Ukraine; second, from a larger survey held in 2010 in six CEE countries, namely Bulgaria, Hungary, Lithuania, Poland, Romania, and Ukraine. Chapter 2 through 4 are devoted to the analysis of data from two SP techniques (DCE and CV defined in Chapter 1), collected in the small-scale survey in Ukraine. Chapters 5 and 6 exploit CV data from the six CEE countries to build potential demand pools and discover the patterns of hypothetical/strategic bias.

In this chapter, we discuss the main findings of the analyses presented in the dissertation as well as their policy and methodology implications. The next section outlines six statements, which summarize the main findings of the dissertation, and elaborates on these statements. The specificities of the research designs and further research strategies are also discussed in this section. The chapter concludes by a general discussion and policy recommendations.

\subsection{Statement discussion}

The six statements presented in this section are divided in two main blocks (three statements in each block). In the first block, the methodological implications of our findings related to 
the use of SP techniques for the assessment of patient payment policies are discussed. The second block of statements relates to the policy implications of our findings for the patient payment policies in the six CEE countries included in the analyses, as well as for the CEE region in general.

\subsubsection{The use of the stated preference techniques for the assessment of patient payments}

A major part of the findings presented in this dissertation are related to the use of two SP techniques, i.e. DCE and CV, for valuing physician services with the aim to assess the patient charges for these services. In particular, we analyze the consistency between the two techniques, the variation in the answering patterns across socio-demographic groups and countries, and the decisions mechanisms employed by respondents. Three statements are formulated based on the results of these analyses.

\section{The consistency between the discrete choice experiment and contingent valuation increases with the importance respondents attach to the presented task.}

In the dissertation, we compare stated WTP estimates obtained from the application of DCE and CV (see Chapter 3) at a disaggregate level (i.e. the comparison of the two WTP estimates per respondent). The DCE and CV data used for the comparison are collected in the same survey (a small-scale survey in Ukraine aiming at the valuation of the physician services). The findings suggest that DCE produces substantially higher estimates than CV. This is in line with previous studies that report disaggregated comparisons between the two techniques (Bijlenga et al., 2011; Ryan and Watson, 2009; van der Pol et al., 2008).

However, unlike previous studies, we find that there is no consistent discrepancy between the two WTP estimates at the disaggregate level, i.e. there is no correlation between the WTP estimates from the two techniques. Our findings suggest that the discrepancy between the two estimates falls with the increase in the stated WTP level, i.e. they converge. This leads to a conclusion that respondents who place a higher value on the changes presented in the valuation tasks (DCE or CV task) discriminate better between the DCE profiles and, hence, are more consistent in their responses. In contrast, those who place a low or zero WTP on the changes, do not discriminate well between the DCE profiles and, consequently, are less confident in their responses or pay less attention to the tasks. 


\section{Chapter 7}

Thus, the strategy to improve the consistency between the WTP estimates obtained from the two SP techniques is to increase the importance of the valuation tasks presented to the respondents (e.g. adaptive choice designs) or to elicit the certainty of the choices made by respondents. Previous literature from different fields of economics has shown that the relevance and completeness of information provided to the respondent affects the stated WTP (e.g. Akcura, 2011; Kartman et al., 1996; Krupnick and Cropper, 1992); however, its effects on the consistency between the two SP techniques has not been studied so far.

Another observation in this dissertation is that WTP estimates are more consistent if smaller changes in the service attributes are assessed. We denote this as a scope effect which implies that the magnitude and variation in the discrepancy between WTP obtained from DCE and $\mathrm{CV}$ are higher if large or total changes rather than marginal changes in quality and access attributes are assessed. Louviere et al. (2010) describe this effect in terms of "the respondent's certainty in their decision” which varies with the scope of the changes valued.

One way is to account for such effect by the application of certain analytical strategies. For example, the application of a non-linear utility specification (Lancsar and Louvierre, 2008; Lancsar et al., 2007; Hanson et al., 2005) might attenuate the scope effect as it allows for diminishing marginal utility and, hence, allows for lower marginal WTP for higher gains in quality and access. Another possibility to account for the differential uncertainty in the individual values is to apply Generalized Mixed Models (e.g. Fiebig et al., 2010), which allow modeling explicitly the heterogeneity in the error scale (its variance) across individuals. A more ambitious way is to apply design strategies that diminish the uncertainty in the respondent's valuation. This will imply presentation of small rather than total or large changes in the attribute values which are most meaningful to a respondent. This is, however, not always possible, especially for qualitative attributes.

It should be noted, however, that our conclusion about higher discrepancy between the two WTP estimates for a bigger scope of attribute changes under assessment, is based on observing changes in WTP for a limited number of profiles (two attributes by two levels). Thus, the relation we observe is discrete and limited. For a more precise assessment of the scope effect on the WTP consistency, the survey design would require the inclusion of a wider range of attribute levels in the valuation tasks, which might also require fewer attributes under consideration. 
At this stage, our conclusions should be interpreted more as a signal for further research on the discrepancy between DCE and CV rather than an indication of exact ways of conducting DCE or CV studies. As suggested by our results, there is room for improving the consistency between DCE and CV. But the strategies to achieve this goal, discussed above, should be studied in detail for their direct effect on the consistency. This would imply the application of a controlled experiment with different groups of respondents being assigned to different versions of the valuation tasks. So far, DCE appears to be unsuitable for a cardinal paradigm (integrating marginal WTP across quality changes to obtain holistic value), neither in policy assessment nor even in cost-benefit analysis, as it is frequently done nowadays. Improving consistency in the WTP estimates by means of design and methodological aspects is a key to understanding the potential of both techniques for policy analysis.

\section{Stated willingness to pay is influenced by the perceived consequences for future policy and by past respondents' experiences with payments and use of care.}

In $\mathrm{CV}$, hypothetical or strategic bias arises when the respondents understate or overstate their true WTP. The empirical strategy for detecting these patterns is a stochastic frontier estimation where the skewness of WTP data is treated as a representation of overstatement (upward skewness) or understatement (downward skewness). In Chapter 6 of this dissertation, we show that the application of the stochastic frontier model of WTP to assess the patterns of hypothetical/strategic bias leads to different conclusions in different countries. Consequently, this bias correction mechanism is not transferrable across countries.

We identify three factors which can explain the variation in the skewness of WTP across countries: the perceived impact on actual policy, the relevance and desirability of the valued changes, and the past experiences with payments. These factors provide an interpretation of our intra-country and cross-country findings.

In countries, where obligatory patient charges are widespread and price increases are considered by the government, respondents might conceal their 'true WTP' due to fear of the consequential price increase. In particular, we found that in Bulgaria where obligatory patient charges for basic services are widely applied and the increase in their levels is under discussion (Radu, 2010; Atanasova et al, 2011), there is a downward skewness and the majority of the WTP answers are concentrated around the upper bound. Moreover, frequent users in Bulgaria, who are prone to pay and thus are most affected by charge increases, are even more likely to have a downward skewness in WTP (understated 'true WTP') compared 


\section{Chapter 7}

to other Bulgarian respondents. Thus, despite the urgent need of quality and access improvements in the public health care sector (Atanasova et al., 2011), the high likelihood of an actual price increase makes some of the respondents understate their 'true' WTP.

In countries where actual price increases are unlikely to happen (obligatory patient charges are limited and their extension is not under discussion), the desire for improvements might lead to overstatement of the 'true WTP'. In Hungary, where obligatory patient charges for basic services were in effect for one year but were abolished (Baji et al., 2011), and the reintroduction is prohibited for a certain period of time, the majority of the WTP answers are rather symmetrically distributed around the 'true WTP'. In the rest of the countries in the study, i.e. Lithuania, Poland, Romania, and Ukraine, where obligatory patient charges for basic services were an unlikely policy action or were limited at the moment of data collection, upward skewness (overstatement of 'true WTP') is observed.

Thus, the strategic answering patterns (under- and overstatement) might be seen as the result of a trade-off a respondent makes between the desirability of changes described in the valuation task (i.e. quality and access improvements) and the likelihood of an actual price increase as a consequence of the survey perceived by the respondent (Hoehn and Randall, 1987). At the same time, the desirability of changes described in the valuation task (i.e. quality and access improvements) might be valued differently in different countries depending on the actual need of such changes (lower in countries where changes are less needed and higher in countries where changes are more needed).

Our findings suggest that there is much more heterogeneity in stated WTP than that which could be explained by observed factors, and part of it is related to the perceived likelihood of payment changes as a consequence of the survey. The application of a stochastic frontier model is a good strategy to discover strategic answering patterns assuming some portion of true WTP in the data. However, such estimations might be improved by the application of a two-tiered stochastic frontier model where simultaneously over- and understatement are allowed. Combining latent class models with stochastic frontier estimation may also allow distinguishing latent population groups with own over- and understatement patterns. From a policy-making perspective, when conservative WTP estimates are sought, downward calibration after estimating stochastic frontier may provide valuable information. Upward calibration, in contrast, should be approached with caution and is not suitable in this framework. As explained for the case of Bulgaria, upward calibration can be used when WTP data are used for prioritizing government investments but not for pricing decisions. 


\section{Different mechanisms underlie the decision to be willing to pay and the level of willingness to pay.}

Based on data from Ukraine (see Chapter 4), we found that different mechanisms drive the decision about being willing to pay and the decision about the level of WTP. The level of WTP, once the decision to be willing to pay is taken, is mostly explained by economic status, i.e. level of monetary income. In the decision to be unwilling to pay, two separate motivations may be distinguished: inability and objection. Inability is related to the perceived income level (despite its monetary value) while the likelihood of objection is not related to the observed economic characteristics of the respondents. We also observe that a person stating inability to pay is less likely to pay in real life for a visit to a physician while stated objection to pay is not related to the respective behavior in real life.

Our results support the current practice in the CV literature of dealing with the decision on being willing to pay and the stated WTP level as separate decision steps. They are also in line with econometric considerations about biased results of the simple Tobit I specification (e.g. Yoo et al., 2001) where only the second stage decision, i.e. level of WTP, is explicitly modeled. Our results suggest that the model specification should allow for the possibility of different factors explaining different decision steps, which is most often done in the CV literature. The first stage decision might be seen as a perceptional issue, while to define the level of WTP, respondents most likely anchor on real budgetary constraint and needs.

The distinction between true zeros and protesters in CV studies is an important analytical choice affecting the results (Boyle, 2003). The question about the motivation for being unable to pay helps in making this distinction. Absence of a relation between stated objection to pay and making payment in real life makes it clear that this motivation indicates that the zero answer is more likely to be a 'protest' and in real life a person would not necessarily attach zero value to the service offered. Reporting inability to pay, in contrast, is associated with not paying for the services consumed in real life (with odds ratio to those who paid of 4.65). Thus, the concentration of 'true' zeros among those who sate inability is much higher. However, for none of the reported motivations (i.e. inability and objection), can an exact conclusion about 'truthfulness' of the zero WTP be made.

Given these results, a conservative strategy for dealing with zero answers would be excluding only pure protesters, i.e. those who object only, from the analysis of the WTP level. In this case, a relatively small part of true zeros among those who object will be ignored, but a small 
part of protesters of those who state inability will be included. The resulting trade-off between omitting true zeros and including protesters is not clear. Further research could focus on design strategies to derive 'true scores' or 'true functions' which represent or predict the propensity of being true zero WTP for the answers stating unwillingness to pay depending on motivation and other covariates. This will require study designs where stated WTP questions would be followed by real life experiments.

\subsubsection{Policy implications for the design of patient payments in Central and Eastern Europe}

This subsection presents the main findings of this dissertation with regards to the policy implications for the CEE countries formulated based on the application of the SP techniques to the assessment of patient payments for the physician services in these countries. We show that unregulated patient payments represent a burden in the CEE countries and argue that with proper policy designs, official patient payments might be an acceptable policy tool.

Current unregulated out-of-pocket payments for physician services represent a substantial financial burden to patients in Central and Eastern European countries.

In Chapter 6, of this dissertation, a comparison of the actual annual expenditure by individuals on outpatient physician services to WTP for such services of high quality and quick access was made. It was shown that in Romania and Ukraine, where informal charges are mostly spread, people spend more on the physician services of common quality than their stated willingness and ability to pay for high quality service. When downward calibration of WTP accounting for possible overstatement is applied, the same is also observed in Lithuania.

This evidence brings up the idea that current unregulated informal and quasi-formal charges that are prevalent in the three countries mentioned above (Murauskiene et al., 2012; Belli, 2003; Danyliv et al., 2012b), are a substantial financial burden to the public. It is recognized that these payments impair access and are regressive as they impose more burden to the lower income groups (Atanasova et al., 2012; Baji et al., 2012b; Szende and Culyer, 2006; Murauskiene et al., 2012; Danyliv et al., 2012b; Lewis, 2000).

It should be noted, however, that in case of Lithuania, Romania and Ukraine, we talk about informal and quasi-formal charges, which are not regulated by the government. These charges do not account for the population's ability to pay and make no formal exemptions for vulnerable groups, thus, being highly regressive in theory and in practice (Szende and Culyer, 
2006; Lewis, 2007). Moreover, out-of-pocket payments might also impose a higher burden for groups with higher health care needs (Golinowska and Tambor, 2012) and, thus, unregulated charges without upper limits may have impoverishing effects in case of major health problems.

Thus, current out-of-pocket patient payments which are not regulated are a burden to patients in CEE countries, especially to low-income and vulnerable groups. They are non-transparent (patients usually do not know what amount they have to pay after service provision) and do not account for patients socio-economic status. Measures to formalize health expenditure should become a policy priority, especially in Lithuania, Romania, and Ukraine, but also in the CEE region in general. Introduction of the official charges (Jakab and Kutzin, 2009; Baschieri and Falkingham, 2006; Barber et al, 2004; Akashi et al., 2004) and severe anticorruption measures (Golinowska, 2010) might be seen as a response to these payments.

Under certain conditions patient payments are an acceptable policy tool in Central and Eastern European countries.

Substantial shares of the population in the six CEE countries included in the study are willing to pay for physician services provided that the services are of adequate quality and access. WTP ranges from $66.5 \%$ in Hungary to $81.43 \%$ in Romania (see Chapter 5). The level of WTP for the physician services is also substantial in all six countries. WTP levels in this study may be more precisely considered as WATP due to the wording of the tasks. Even with this correction, the mean and median levels of WATP represent rather high amounts when compared to the indication of the service cost. They are higher than the cost of services in Hungary, Romania, and Ukraine, nearly equal to service cost in Bulgaria, and equal to a rather substantial part of the service cost in Poland and Lithuania. Even if downward calibration of the stated WATP is applied (see Chapter 6) the mean WATP still represents not less than $50 \%$ of the service cost.

The amount of patient charges for basic services is usually set so that it does not cover the cost of the service, but only some share. Given that the level of WATP is comparable to at least half of the service cost, patient payments might have a good potential in all six countries in the study. The demand pool modeling (see Chapter 5) also demonstrates that low copayments of up to $€ 2.5$ should not represent a high burden in the five countries, especially if exemptions for vulnerable groups are designed. Ukrainian consumers would react more 


\section{Chapter 7}

abruptly to the introduction of the small fee of up to $€ 2.5$ and, hence, a more sensitive study tool should be applied to better elicit the preferences within this payment interval.

All types of WTP modeling applied in this dissertation, i.e. direct WTP (Chapter 4), survival analysis (Chapter 5), and stochastic frontier modeling (Chapter 6), yield similar conclusions about socio-demographic and economic drivers of WTP. WTP and, consequently demand pools, are related to household income in five countries, but less in Ukraine. There, the wellestablished payment practices (informal and quasi-formal payments) (Gryga et al., 2010) perhaps eliminate some variation in stated WTP due to common ideas in society about a fair price. In Ukraine, elderly people are less likely to be willing to pay and this tendency might be explained by the remnants of the communist views in this group as they still feel nostalgic about the old times of free social benefits (Survey, 2011). The observed demand differences across place of residence, which are observed in Hungary, but also in Bulgaria, Lithuania, and Poland are in line with the theoretical and empirical literature suggesting price discrimination by geographical area (McPake, 1993).

Thus, the WTP data in all six countries suggest that payments might be seen as an acceptable policy tool in case adequate polices for protecting vulnerable groups are implemented, informal charges are eradicated, and feasible fee levels are established. The vulnerable groups subject to fee exemption might be based on household income level in Bulgaria, Lithuania, Hungary, Poland, and Romania. In Ukraine, age should also be seen as an exemption criterion. A regional (place of residence) principle for fee segregation is harder to apply. The higher values expressed by dwellers of small cities in Bulgaria, Hungary, and Lithuania might actually be related to the relatively more difficult access to these services than in urban areas. This is the case in most countries despite the level of development (World Health Organization, 2008).

Special attention in the patient payment policy should be devoted to the fee for direct referrals avoiding primary physician contact (GP or her counterparts). Based on DCE and CV data from Ukraine (Chapter 2 and 3), it is shown that there is no explicit preference in the population for addressing a GP or a medical specialist directly. However, groups with a higher socio-economic status might prefer direct referral. Given the positive effect of the gatekeeping in terms of system efficiency (González, 2010; Zielinski et al., 2008; Starfield, 1992; Schwenkglenks et al., 2006), it might be a good strategy to impose penalizing fees for direct referrals, as it is in most CEE countries. With a very slight preference towards direct referral 
(around $€ 0.6$ per visit in Ukraine) the fee that will efficiently reduce this practice should not be high.

A rather substantial challenge for the introduction of official patient charges is the existence of informal payments. There is a debate in the literature whether formal charges might substitute informal payments (Jakab and Kutzin, 2009; Baschieri and Falkingham, 2006; Barber et al, 2004; Akashi et al., 2004) or whether they will impose a double burden on a patient with regressive outcomes (e.g. Atanasova et al., 2012; Baji et al., 2012a; Rückert et al., 2008; Wagstaff and van Doorslaer, 1992). Based on the analysis of Ukrainian data in this dissertation (Chapter 2), it is expected that there might also be a problem on the patient's side in switching to formal charges as there is no explicit preference for paying formally over paying informally. This may transform into resistance to the introduction of official charges as patients might continue to pay informally if this is required by health care providers. The new payments may be considered not as an alternative, but as additional compulsory levies. More than that, official charges might be perceived as detached from personal communication with the physician and unlike informal payments not assuring quicker access and better quality of care. The positive side is that no reverse preference is observed and this is a signal that public attitudes can be changed. Therefore, the implementation of official charges should be supported by active measures to build social resistance towards informal payments, which is absent now.

People in Central and Eastern European countries are willing to pay for quality and access, so patient payment policies should be tied to quality and access improvements.

WTP for physician services is driven by the quality level of the services. Based on CV data from Ukraine, we outline in Chapter 4 that people place a high economic value on quality and access improvements. Thus, patient charges should be implemented together with effective investment policies targeted at improving quality and access. The probability to object to pay for these services is mostly explained by low quality/access characteristics. Additionally, the likelihood of the ability to pay and the level of positive WTP are positively and strongly related to the quality/access profile. Combined with the evidence that Ukrainians in general are not satisfied with the quality of care they receive (Gorshenin Institute, 2011), these findings underline the necessity of quality/access improvements in health care and patient charges may generate additional funds for these purposes. 


\section{Chapter 7}

Societal benefits (expressed through the increase in WTP) gained from quality improvements in the state of medical equipment, maintenance of the physician office, and reduction of waiting time is $16.5 \mathrm{UAH}$ ( $€ 1.43$ ) per visit to a GP and $23.0 \mathrm{UAH}$ ( $€ 1.99$ ) per visit to a medical specialist (for December 2009). This can be regarded as the investment potential in the increased quality of the services. The average salary in the heath sector in 2009 was $€ 135$, and salaries constitute between $75 \%$ and $80 \%$ of the public health facilities. By indirect evidence the cost of service does not exceed $€ 3-€ 5$ per physician consultation (reimbursement tariff for private insurance companies by Tverdokhlib and Horshkov, 2011). Thus, it is clear that even these, moderate in absolute numbers, investment amounts make a substantial share of the current cost of services.

Quality improvements in Ukraine may not be achieved with infrastructural changes only (i.e. capital investments, organizational changes, better training). Physicians are the main quality agents in this case, but they are severely underpaid. This is one of the main reasons of the current widespread informal payments aimed to compensate for the lack of remuneration and assure better quality and quicker access to care. Thus, the official remuneration should be considered as one of the main quality investment components. Failure to account for physician needs in this process may cause resistance to changes and a continuation of selective care based on informal contributions.

In Chapter 2, the relative importance of the quality and access characteristics of physician services in Ukraine is elicited through DCE. Apart from clinical excellence, which is the main quality indicator per se, the social quality and access characteristics play an important role in the choice of a physician. Above all, the most highly valued characteristic relates to the interpersonal aspects of care. A number of studies from other countries also find interpersonal aspects the most important value component (e.g. Vick and Scott, 1998; Coulter, 1995; Leon, 2003; review by Wensing et al., 1998).

In our study, the interpersonal aspects were represented by the attribute 'attitude of the medical staff'. However, this is a broader concept which implies the need for attention, information, and involvement in care. The preference for these aspects is even more prominent among elderly people. All these issues are fully neglected now in the care provision process in Ukraine, and in public facilities patients are often treated arrogantly, not to mention completeness of the information and involvement in decisions (Gryga et al., 2010). Elderly people in Ukraine lack special care programs designed for their needs, and most often are treated as 'close to dying' and thus as such that do not require much attention (Bezrukov, 
2003). Even informal payments cannot always assure a sufficient level of attention due to the lack of regulation (e.g. Vian et al., 2006). Thus, in the investment agenda, the training of medical staff in communicational skills should be given priority, especially for those specialists who provide care for elderly. Ideally, standards of care should establish minimal requirements to communication with patients. Feedback procedures should also enable that patients realize their rights to decent treatment and involvement in the decisions about their own health.

Still, people highly value the clinical efficiency and safety of care, which was represented by the state of medical equipment. Preferences for good maintenance of the physician's office and reduced travel and waiting time are approximately of the same magnitude. Therefore, policy interventions aimed to improve access and state of medical facilities should be given the same priority, and be ranked lower compared to improvements in interpersonal aspects of care and medical technology. The societal benefits gained from the improvement of some of these characteristics were mentioned above and may be considered as investment potential. Residents of rural areas lack most of these and, hence, place a higher value on wellmaintained premises which underlines the necessity of redistribution of capital investment to rural areas.

\subsection{Final conclusion}

This dissertation has shown, as anticipated in objective 7 of this dissertation, that despite the limitations discussed in Chapter 1, SP techniques provide useful information to policy makers which may help them in designing patient payment policies that correspond well to public preferences. This is especially valuable in CEE countries with a lack of experience with actual patient payments as there no data are available on actual reactions to official payment regimes. Values obtained via DCE could be used for the estimation of relative preferences, i.e. marginal WTP indicates only the relative importance of the quality and access characteristics. CV may be used for a holistic assessment of WTP, but still it is not a direct indication of the actual fee as it might be subject to hypothetical bias. On the other hand, in the absence of actual behavior data, CV measures might be used as an indication of the reservation price and, hence, for measuring possible consumer reactions to the fee variations. Country specific CV instruments may better account for meaningful payment differences, which are also related to the income level and disparities in the country. 


\section{Chapter 7}

In this dissertation, we addressed three methodological issues of the application of SP to the assessment of patient payment policies for physician services, i.e. consistency between the DCE and CV estimates of WTP (objective 3), the respondents' decision process (the outcome of addressing objective 2), and the strategic answering patterns (objective 5). We also discussed three policy-related aspects based on our findings, i.e. the burden of current unregulated payments given the level of quality of the services (objective 6), the potential consumption reaction to the introduction of the patient charges (objective 4 and partly objective 2), and public preferences for quality and access improvements of the physician services (objective 1).

The question of the correspondence and consistency between WTP estimates obtained from DCE and CV methods was addressed. Lack of consistency between them implies that obtaining holistic values of the services by integrating the marginal WTP across changes in attributes might be an erroneous approach, which nonetheless seems to be taken for granted by some researchers as was shown in Chapter 1. On the other hand, the consistency between the two approaches may be increased by enhancing the relevance of the valuation tasks to respondents, i.e. relevant attributes, meaningful attribute levels. The development of such strategies (e.g. accounting for valuation certainty, adaptive choice designs etc.) with thorough assessment of their impact on the consistency might eventually lead to a rather clear link between marginal and holistic WTP.

In terms of modeling holistic WTP obtained in a CV task, the decision about stating positive WTP and about choosing its level are clearly separate decisions. Moreover, there might be a way to address the problem of distinction between the true zeros and protesters. In this dissertation, the distinction between the objection and inability motives was done and the latter seems more likely to be true zeros than the former. Further, techniques allowing for the assessment of the 'true' zero propensities based on the stated motivation are necessary in order to bring CV estimates closer to the real-life WTP. Simply ignoring the problem of true zeros might lead to biased estimates.

The results of this dissertation also reveal that the strategic answering patterns in SP are related to the country context in terms of experience with and potential consequences for patient payments for the services under valuation. In a policy-making context, conservative WTP estimates may be obtained by the application of downward calibration techniques, e.g. based on stochastic frontier estimation. In countries where potential price consequences are likely, respondents might be concealing their true WTP. However, upward calibration is 
unlikely to be adopted by policymakers. Inclusion of latent classes in the stochastic frontier estimation might separate WTP heterogeneity due to socio-demographic differences from the one due to strategic answering.

Form a policy perspective, the results of this dissertation confirm that the current payment practices for physician services, which include unregulated informal and quasi-formal payments, represent a financial challenge to the CEE countries. In countries where they are most prevalent, there is a mismatch between public preferences for physician services and expenditure on them. That is the current level of quality and access does not correspond to the level of the charges born by patients. Therefore, measures targeted at both: a reduction of the informal charges and an increase in the quality of the services, would be beneficial.

The decision on the acceptance of official charges (co-payments) for physician services is a multidimensional issue and involves broader considerations than just the results of modeling stated WTP. We attempted to show what would happen if they were accepted in CEE countries, based on the stated WTP. Our results demonstrate a potential consumption reaction to the introduction of certain levels of charges and the difference in the speed of this reaction in population groups. We find that on average rather substantial shares of the population in CEE countries are willing to pay, and by conservative estimates, the average WTP covers at least half of the service cost in these countries. The least harmful but efficiency enhancing strategy would be charges for self-referrals to specialists (avoiding the GP). In countries with well-established informal payment practices, however, formal charges would meet opposition unless public opinion, which tolerates informal payments at present, is changed.

Finally, this dissertation makes a point of the necessity and structure of quality and access improvements of physician services in Ukraine. People are willing to pay for quality and access improvements in these services and the amounts represent a substantial part of the service cost. Notably, the highest value component in the service provision is the interpersonal aspects of care and, hence they should get most attention in the development of health care reform strategies. Nowadays however, most of the attention of Ukrainian policy makers is concentrated on infrastructural issues, such as maintenance and equipment of facilities (e.g. $\mathrm{MOH}, 2010$ ) while the attitude of medical staff to patients is neglected. The results of this dissertation suggest that the prioritization should be changed or, at least, interpersonal aspects should appear on the agenda (not only in Ukraine but also in other CEE countries). 


\section{Chapter 7}

Our study demonstrates the way SP may be used to study potential effects of patient charges on health care consumption in non-existent markets. Patient charges for physician services, though ignored and dreaded in CEE countries, may be an efficient policy tool in light that people in these countries are ready and able to pay substantial amounts formally if the services are provided with adequate quality and quick access. The charges should be however supplemented with adequate exemption mechanisms to secure access to essential health care services. 


\section{References}

Aarva, P., Ilchenko, I., Gorobets, P., \& Rogacheva, A. (2009). Formal and informal payments in health care facilities in two Russian cities, Tyumen and Lipetsk. Health Policy and Planning, 24(5), 395-405.

Adamowicz, W., Boxall, P., Williams, M., \& Louviere, J. (1998). Stated preference approaches for measuring passive use values: choice experiments and contingent valuation. American Journal of Agricultural Economics, 80(1), 64-75.

Adamowicz, W., Louviere, J., \& Williams, M. (1994). Combining revealed and stated preference methods for valuing environmental amenities. Journal of Environmental Economics and Management, 26(3), 271-292.

Akashi, H., Yamada, T., Huot, E., Kanal, K., \& Sugimoto, T. (2004). User fees at a public hospital in Cambodia: effects on hospital performance and provider attitudes. Social Science \& Medicine, 58(3), 553-564.

Akcura, E. (2011). Information Effects in Valuation of Electricity and Water Service Attributes Using Contingent Valuation. (Working paper No. 1156). Retrieved from Cambridge Working Papers in Economics, Faculty of Economics, University of Cambridge: https://www.repository.cam.ac.uk/handle/1810/242026

Albreht, T., Turk, E., Toth, M., Ceglar, J., Marn, S., Pribaković Brinovec, R., \& Schäfer, M. (2009). Slovenia: Health system review. Health Systems in Transition, 11(3), 1-168.

Arrow, K., Solow, R., Portney, P., Leamer, E., Radner, R., \& Schuman, H. (1993, January 11). Report of the NOAA panel on contingent valuation. Washington, DC: National Oceanic and Atmospheric Administration.

Arsenijevic, J., Pavlova, M., \& Groot, W. (2012). Measuring the catastrophic and impoverishing effect of household health care spending in Serbia. Social Science \& Medicine, 78, 17-25.

Asgary, A. (2012). Assessing households' willingness to pay for an immediate pandemic influenza vaccination programme. Scandinavian Journal of Public Health, 40(5), 412417. 


\section{References}

Atanasova, E., Pavlova, M., Moutafova, E., Rechel, B., \& Groot, W. (2012). Out-of-pocket payments for health care services in Bulgaria: financial burden and barrier to access. The European Journal of Public Health. Doi: 10.1093/eurpub/cks169.

Atanasova, E., Pavlova, M., Velickovski, R., Nikov, B., Moutafova, E., \& Groot, W. (2011). What have 10 years of health insurance reforms brought about in Bulgaria? Re-appraising the Health Insurance Act of 1998. Health Policy, 102(2-3), 263-269.

Augurzky, B., Bauer, T. K., \& Schaffner, S. (2006). Copayments in the German Health System-Do They Work? (RWI Discussion Paper No. 43). iHEA 2007 6th World Congress: Explorations in HealthEconomics Paper. DOI: 10.2139/ssrn.928914

Baji, P., Pavlova, M., Gulacsi, L., \& Groot, W. (2012a). Changes in Equity in Out-of-pocket Payments during the Period of Health Care Reforms: Evidence from Hungary. International Journal for Equity in Health, 11(1), 1-11.

Baji, P., Pavlova, M., Gulácsi, L., Zsófia, H. C., \& Groot, W. (2012b). Informal payments for healthcare services and short-term effects of the introduction of visit fee on these payments in Hungary. The International Journal of Health Planning and Management, 27(1), 63-79.

Baji, P., Pavlova, M., Gulácsi, L., \& Groot, W. (2011). User fees for public health care services in Hungary: Expectations, experience, and acceptability from the perspectives of different stakeholders. Health Policy, 102(2), 255-262.

Balabanova, D., \& McKee, M. (2002). Understanding informal payments for health care: the example of Bulgaria. Health Policy, 62(3), 243-273.

Balabanova, D., McKee, M., Pomerleau, J., Rose, R., \& Haerpfer, C. (2004). Health service utilization in the former Soviet Union: evidence from eight countries. Health Services Research, 39(6p2), 1927-1950.

Barber, S., Bonnet, F., \& Bekedam, H. (2004). Formalizing under-the-table payments to control out-of-pocket hospital expenditures in Cambodia. Health Policy and Planning, 19(4), 199-208.

Barmina, G. (2009). A step towards effective medical care - development of the primary care. (in Ukrainian) Apteka.ua. Retrieved from http://www.apteka.ua/article/8421

Barros, P. P., Machado, S. R., \& Simões, J. d. A. (2011). Portugal Health system review. Health Systems in Transition, 13(4). 
Baschieri, A., \& Falkingham, J. (2006). Formalizing informal payments: the progress of health reform in Kyrgyzstan. Central Asian Survey, 25(4), 441-460.

Belli, P. (2003). Formal and informal household spending on health: a multicountry study in Central and Eastern Europe. (Final Report) Harvard School of Public Health: Cambridge.

Belli, P., Gotsadze, G., \& Shahriari, H. (2004). Out-of-pocket and informal payments in health sector: evidence from Georgia. Health Policy, 70(1), 109-123.

Berki, S. E., \& Ashcraft, M. L. (1980). HMO enrollment: who joins what and why: a review of the literature. The Milbank Memorial Fund Quarterly. Health and Society, 58(4), 588632.

Bezrukov, V.V. (2003). Case-Study Ukraine. In J. Brodsky, J. Habib, \& M. Hirschfeld (Eds.), Long-term Care in Developing Countries: Ten Country Case Studies. World Health Organization.

Bijlenga, D., Bonsel, G. J., \& Birnie, E. (2011). Eliciting willingness to pay in obstetrics: comparing a direct and an indirect valuation method for complex health outcomes. Health Economics, 20(11), 1392-1406.

Blackburn, M., Harrison, G. W., \& Rutström, E. E. (1994). Statistical bias functions and informative hypothetical surveys. American Journal of Agricultural Economics, 76(5), 1084-1088.

Blamey, R., Bennett, J. W., \& Morrison, M. (1999). Yea-saying in contingent valuation surveys. Land Economics, 75(1), 126-141.

Blumenschein, K., Johannesson, M., Yokoyama, K., \& Freeman, P. (2001). Hypothetical versus real willingness to pay in the health care sector: results from a field experiment. Value in Health, 4(2), 79-79.

Bobinac, A., van Exel, N. J., Rutten, F. F., \& Brouwer, W. B. (2012a). Get more, pay more? An elaborate test of construct validity of willingness to pay per QALY estimates obtained through contingent valuation. Journal of Health Economics, 31(1), 158-168.

Bobinac, A., van Exel, N. J., Rutten, F. F., \& Brouwer, W. B. (2012b). Valuing qaly gains by applying a societal perspective. Health Economics,22(10), 1272-1281. 


\section{References}

Boxall, P. C., Adamowicz, W. L., Swait, J., Williams, M., \& Louviere, J. (1996). A comparison of stated preference methods for environmental valuation. Ecological Economics, 18(3), 243-253.

Boyle, K. (2003). Contingent Valuation in Practice. In P. Champ, K. Boyle \& T. Brown (Eds.), A Primer on Nonmarket Valuation (Vol. 3, pp. 111-169): Springer Netherlands.

Brandt, S., Vasquez Lavin, F., \& Hanemann, M. (2012). Contingent valuation scenarios for chronic illnesses: the case of childhood asthma. Value in Health, 15(8), 1077-1083.

Brau, R., \& Lippi Bruni, M. (2008). Eliciting the demand for long-term care coverage: a discrete choice modelling analysis. Health Economics, 17(3), 411-433.

Bredenkamp, C., Mendola, M., \& Gragnolati, M. (2011). Catastrophic and impoverishing effects of health expenditure: new evidence from the Western Balkans. Health Policy and Planning, 26(4), 349-356.

Brekke, K. R., Nuscheler, R., \& Straume, O. R. (2007). Gatekeeping in health care. Journal of Health Economics, 26(1), 149-170.

Bridges, J. F., Hauber, A. B., Marshall, D., Lloyd, A., Prosser, L. A., Regier, D. A., Johnson, R., \& Mauskopf, J. (2011). Conjoint analysis applications in health - a checklist: A report of the ISPOR good research practices for conjoint analysis task force. Value in Health, 14(4), 403-413.

Brown, T. C., \& Gregory, R. (1999). Why the WTA-WTP disparity matters. Ecological Economics, 28(3), 323-335.

Bryndová, L., Pavloková, K., Roubal, T., Rokosová, M., \& Gaskins, M. (2009). Czech Republic. Health system review. Health Systems in Transition, 11(1), 1-122.

Carrin, G., \& van Dael, J. (1990). An empirical model of the demand for health care in Belgium. In G. Duru \& J. H. P. Paelinck (Eds.), Econometrics of Health Care (Vol. 20, pp. 59-78): Springer Netherlands.

Carroll, F. E., Al-Janabi, H., Flynn, T., \& Montgomery, A. A. (2013). Women and their partners' preferences for Down's syndrome screening tests: a discrete choice experiment. Prenatal Diagnosis, 33(5), 449-456.

Carson, R., \& Groves, T. (2007). Incentive and informational properties of preference questions. Environmental and Resource Economics, 37(1), 181-210. 
Carson, R. T. (2000). Contingent valuation: a user's guide. Environmental Science \& Technology, 34(8), 1413-1418.

Carson, R. T., Mitchell, R. C., Hanemann, M., Kopp, R. J., Presser, S., \& Ruud, P. A. (2003). Contingent valuation and lost passive use: damages from the Exxon Valdez oil spill. Environmental and Resource Economics, 25(3), 257-286.

Carson, R. T., Wilks, L., \& Imber, D. (1994). Valuing the preservation of Australia's Kakadu conservation zone. Oxford Economic Papers, 46, 727-749.

Chen, L. C., Schafheutle, E. I., \& Noyce, P. R. (2009). The impact of nonreferral outpatient co-payment on medical care utilization and expenditures in Taiwan. Research in Social \& Administrative Pharmacy, 5(3), 211-224.

Cheraghi-Sohi, S., Hole, A. R., Mead, N., McDonald, R., Whalley, D., Bower, P., \& Roland, M. (2008). What patients want from primary care consultations: a discrete choice experiment to identify patients' priorities. The Annals of Family Medicine, 6(2), 107-115.

Cheraghi-Sohi, S., Bower, P., Mead, N., McDonald, R., Whalley, D., \& Roland, M. (2006). What are the key attributes of primary care for patients? Building a conceptual 'map'of patient preferences. Health Expectations, 9(3), 275-284.

Cherkin, D., Grothaus, L., \& Wagner, E. (1992). Is magnitude of co-payment effect related to income? Using census data for health services research. Social Science \& Meicine, 34(1), $33-41$.

Chestnut, L. G., Keller, L. R., Lambert, W. E., \& Rowe, R. D. (1996). Measuring heart patients' willingness to pay for changes in angina symptoms. Medical Decision Making : An International Journal of the Society for Medical Decision Making, 16(1), 65-77.

Chiappori, P. A., Durand, F., \& Geoffard, P. Y. (1998). Moral Hazard and the Demand for Physician Services: First Lessons from a French Natural Experiment. European Economic Review, 42(3-5), 499-511.

Ching, P. (1995). User fees, demand for children's health care and access accross income groups: The Philippine case. Social Science \& Medicine, 41(1), 37-46.

Clark, M., Moro, D., \& Szczepura, A. (2009). Balancing patient preferences and clinical needs: Community versus hospital based care for patients with suspected DVT. Health Policy, 90(2-3), 313-319. 


\section{References}

Cockx, B., \& Brasseur, C. (2003). The demand for physician services. Journal of Health Economics, 22(6), 881-913.

Committee on Economic Reforms at the President of Ukraine. 2010. Wealthy society, competitive economy, efficient state. Program of economic reforms for 2010-2014. (in Ukrainian). Retrieved from www.president.gov.ua/docs/Programa_reform FINAL_1.pdf.

Corso, P. S., Ingels, J. B., \& Roldos, M. I. (2013). A comparison of willingness to pay to prevent child maltreatment deaths in ecuador and the United States. International Journal of Environmental Research and Public Health, 10(4), 1342-1355.

Coulter, A. (1995). Shifting the balance from secondary to primary care. British Medical Journal, 311(7018), 1447-1448.

Cropper, M. L., Haile, M., Lampietti, J., Poulos, C., \& Whittington, D. (2004). The demand for a malaria vaccine: evidence from Ethiopia. Journal of Development Economics, 75(1), 303-318.

Dalmau-Matarrodona, E. (2001). Alternative approaches to obtain optimal bid values in contingent valuation studies and to model protest zeros. Estimating the determinants of individuals' willingness to pay for home care services in day case surgery. Health Economics, 10(2), 101-118.

Damme, W. V., Leemput, L. V., Hardeman, W., \& Meessen, B. (2004). Out-of-pocket health expenditure and debt in poor households: evidence from Cambodia. Tropical Medicine \& International Health, 9(2), 273-280.

Danyliv, A., Pavlova, M., Gryga, I., \& Groot, W. (2013). Willingness to pay for physician services at a primary contact in Ukraine: Results of a contingent valuation study. BMC Health Services Research, 13(1), 208.

Danyliv, A., Pavlova, M., Gryga, I., \& Groot, W. (2012a). Willingness to pay for physician services: Comparing estimates from a discrete choice experiment and contingent valuation. Society and Economy, 34(2), 339-357.

Danyliv, A., Stepurko, T., Gryga, I., Pavlova, M., \& Groot, W. (2012b). Is there a place for the patient in the Ukrainian health care system? Patient payment policies and investment priorities in health care in Ukraine. Society and Economy, 34(2), 273-291. 
Delcheva, E., Balabanova, D., \& McKee, M. (1997). Under-the-counter payments for health care: Evidence from Bulgaria. Health Policy, 42(2), 89-100.

Diener, A., O'Brien, B., \& Gafni, A. (1998). Health care contingent valuation studies: a review and classification of the literature. Health Economics, 7(4), 313-326.

Dixon, S., Shackley, P., Bonham, J., \& Ibbotson, R. (2012). Putting a value on the avoidance of false positive results when screening for inherited metabolic disease in the newborn. Journal of Inherited Metabolic Disease, 35(1), 169-176.

Donaldson, C., Jones, A. M., Mapp, T. J., \& Olson, J. A. (1998). Limited dependent variables in willingness to pay studies: applications in health care. Applied Economics, 30(5), 667677.

Doorslaer van, E., O'Donnell, O., Rannan-Eliya, R. P., Somanathan, A., Adhikari, S. R., Garg, C. C., Harbianto, D., Herin, A., Huq, M., Ibragimova, S., Karan, A., Ng, CW., Pande, B, Rachelis, R., Tao, S., Tin, K., Tisayaticom, K., Trisnantoro, L., Vasavid, C., \& Zhao, Y. (2006). Effect of payments for health care on poverty estimates in 11 countries in Asia: an analysis of household survey data. The Lancet, 368(9544), 1357-1364, Doi: 10.1016/S0140-6736(06)69560-3.

Doorslaer van, E. (1984). The effects of cost sharing on the demand for prescription drugs in Belgium. Acta Hospitalia, 24(3), 69-81.

Drummond, M., \& Towse, A. (2012). Is it time to reconsider the role of patient co-payments for pharmaceuticals in Europe? The European Journal of Health Economics, 13(1), 1-5.

Edelényi, M., Tóth, A., \& Neumann, L. (2008). Majority vote 'yes’ in referendum to abolish medical and higher education fees. EIROnline, 4. Retrieved from http://www.eurofound.europa.eu/eiro/2008/04/articles/hu0804029i.htm.

Ensor, T. (2004). Informal payments for health care in transition economies. Social Science \& Medicine, 58(2), 237-246.

Ensor, T., \& Savelyeva, L. (1998). Informal payments for health care in the Former Soviet Union: some evidence from Kazakstan. Health Policy and Planning, 13(1), 41-49.

Ensor, T., \& Thompson, R. (2011). Unofficial payments in low-and middle-income countries. In A. Jones (Eds.), The Elgar Companion to Health Economics, Edward Elgar Publishing Limited: UK, 154-163. 


\section{References}

Erichsen, L., \& Brockhoff, P. B. (2004). An application of latent class random coefficient regression. Advances in Decision Sciences, 8(4), 247-260.

Escarce, J. J., Kapur, K., Joyce, G. F., \& Van Vorst, K. A. (2001). Medical care expenditures under gatekeeper and point-of-service arrangements. Health Services Research, 36(6 Pt 1), 1037.

Espin, J., \& Rovira, J. (2007). Analysis of differences and commonalities in pricing and reimbursement systems in Europe. (Final Report). Brussels: DG Enterprise and Industry of the European Commission. Retrieved from http://ec.europa.eu/enterprise/sectors/healthcare/files/docs/study_pricing_2007/andalusia n_school_public_health_report_pricing_2007_en.pdf

Essers, B. A., Dirksen, C. D., Prins, M. H., \& Neumann, H. A. (2010). Assessing the public's preference for surgical treatment of primary basal cell carcinoma: A discrete-choice experiment in the south of the Netherlands. Dermatologic Surgery, 36(12), 1950-1955.

Ethier, M. C., Regier, D. A., Tomlinson, D., Judd, P., Doyle, J., Gassas, A., Naqvi, A., \&. Sung, L. (2012). Perspectives toward oral mucositis prevention from parents and health care professionals in pediatric cancer. Support Care Cancer, 20(8), 1771-1777.

Falkingham, J. (2004). Poverty, out-of-pocket payments and access to health care: evidence from Tajikistan. Social Science \& Medicine, 58(2), 247-258.

Ferris, T. G., Chang, Y., Blumenthal, D., \& Pearson, S. D. (2001). Leaving gatekeeping behind-effects of opening access to specialists for adults in a health maintenance organization. New England Journal of Medicine, 345(18), 1312-1317.

Fiebig, D. G., Keane, M. P., Louviere, J., \& Wasi, N. (2010). The generalized multinomial logit model: Accounting for scale and coefficient heterogeneity. Marketing Science, 29(3), 393-421.

Finn, A., \& Louviere, J. J. (1992). Determining the appropriate response to evidence of public concern: the case of food safety. Journal of Public Policy \& Marketing, 11(2), 12-25.

Flynn, T. N. (2010). Valuing citizen and patient preferences in health: recent developments in three types of best-worst scaling. Expert Review of Pharmacoeconomics \& Outcomes Research, 10(3), 259-267. 
Flynn, T. N., Louviere, J. J., Peters, T. J., \& Coast, J. (2007). Best-worst scaling: what it can do for health care research and how to do it. Journal of Health Economics, 26(1), 171189.

Foreit, J. R., \& Fleischman Foreit, K. G. F. (2003). The reliability and validity of willingness to pay surveys for reproductive health pricing decisions in developping countries. Health Policy, 63(1), 37-47.

Foster, V., Bateman, I. J., \& Harley, D. (1997). Real and hypothetical willingness to pay for environmental preservation: A non-experimental comparison. Journal of Agricultural Economics, 48(1-3), 123-137.

Fox, J. A., Shogren, J. F., Hayes, D. J., \& Kliebenstein, J. B. (1998). CVM-X: Calibrating contingent values with experimental auction markets. American Journal of Agricultural Economics, 80(3), 455-465.

Frew, E. J., Whynes, D. K., \& Wolstenholme, J. L. (2003). Eliciting willingness to pay: comparing closed-ended with open-ended and payment scale formats. Medical Decision Making, 23(2), 150-159.

Gaál, P., Evetovits, T., \& McKee, M. (2006). Informal payment for health care: evidence from Hungary. Health Policy, 77(1), 86-102.

Gaál, P., Jakab, M., \& Shishkin, S. (2010). Strategies to Address Informal Payments for Health Care. In J. Kutzin, C. Cashin \& M. Jakab (Eds.), Implementing Health Financing Reform. Lessons from Countries in Transition. Copenhagen: European Observatory on Health Systems and Policies / World Health Organization Regional Office for Europe.

Gaál, P., \& McKee, M. (2005). Fee-for-service or donation? Hungarian perspectives on informal payment for health care. Social Science \& Medicine, 60(7), 1445-1457.

Gaál, P., Szigeti, S., Csere, M., Gaskins, M., \& Panteli, D. (2011). Hungary Health system review. Health Systems in Transition, 13(5), 1-266.

Georgieva, L., Salchev, P., Dimitrova, R., Dimova, A., \& Avdeeva, O. (2007). Bulgaria Health system review. Health Systems in Transition, 9(1), 1-156.

Gertler, P., \& van der Gaag, J. (1990). The willingness to pay for medical care: evidence from two developing countries. Published for the World Bank [by] Johns Hopkins University Press. 


\section{References}

Gidengil, C., Lieu, T. A., Payne, K., Rusinak, D., Messonnier, M., \& Prosser, L. A. (2012). Parental and societal values for the risks and benefits of childhood combination vaccines. Vaccine, 30(23), 3445-3452.

Gjorgjev, D., Bacanovic, A., Cicevalieva, S., Sulevski, Z., \& Grosse-Tebe, S. (2008). The former Yugoslav Republic of Macedonia: Health system review. Health Sysytems in Transition, 8(2), 1-98.

Glenngard, A. H., Hjelmgren, J., Thomsen, P. H., \& Tvedten, T. (2012). Patient preferences and willingness-to-pay for ADHD treatment with stimulants using discrete choice experiment (DCE) in Sweden, Denmark and Norway. Nordic Journal of Psychiatry, 67(5), 351-359.

Goldar, B., \& Misra, S. (2001). Valuation of environmental goods: correcting for bias in contingent valuation studies based on willingness-to-accept. American Journal of Agricultural Economics, 83(1), 150-156.

Golinowska, S. (2010). Informal payments in health care. Perspective and evidence form Poland. Zeszyty Naukowe Ochrony Zdrowia. Zdrowie Publiczne i Zarzadzanie [Public Health and Management], 8(1), 12-28.

Golinowska, S., \& Sowa, A. (2006, 19-21 January). Investing in Health Institutions in Transition Countries. Paper presented at the Seventh Annual Global Development Conference "Institutions and Development: At the Nexus of Global Change”, St. Petersburg.

Golinowska, S., \& Tambor, M. (2012). Out-of-pocket payments on health in Poland: Size, tendency and willingness to pay. Society and Economy, 34(2), 253-271.

González, P. (2010). Gatekeeping versus direct-access when patient information matters. Health economics, 19(6), 730-754.

Gorshenin Institute. (2011). 80\% of Ukrainians are not satisfied with the quality of medical care. (in Ukrainian). Unian. Retrieved from http://health.unian.net/ukr/detail/224066

Gotsadze, G., Chechulin, Y., Galayda, V., Lekhan, V., Chanfreau, C., \& Dmytraczenko, T. (2006). Ukraine National Health Accounts 2003-2004 (Vol. 1 and 2).

Gotsadze, G., \& Gaal, P. (2010). Coverage Decisions: Benefit Entitlements and Patient Cost Sharing. In J. Kutzin, C. Cashin \& M. Jakab (Eds.), Implementing Health Financing 
Reform Lessons from Countries in Transition. Copenhagen: European Observatory on Health Systems and Policies/World Health Organization Regional Office for Europe.

Granovetter, M. (1985). Economic action and social structure: the problem of embeddedness. American journal of sociology, 481-510.

Greene, W. (2005). Reconsidering heterogeneity in panel data estimators of the stochastic frontier model. Journal of Econometrics, 126(2), 269-303.

Groot, W., \& van den Brink, H. M. (2007). Optimism, pessimism and the compensating income variation of cardiovascular disease: A two-tiered quality of life stochastic frontier model. Social Science \& Medicine, 65(7), 1479-1489.

Groot, W., Van Den Brink, H. M., \& Plug, E. (2004). Money for health: The equivalent variation of cardiovascular diseases. Health Economics, 13(9), 859-872.

Grumbach, K., Selby, J. V., Damberg, C., Bindman, A. B., Quesenberry Jr, C., Truman, A., \& Uratsu, C. (1999). Resolving the gatekeeper conundrum. The Journal of the American Medical Association, 282(3), 261-266.

Gryga, I., Stepurko, T., Danyliv, A., Gryga, M., Lynnyk, O., Pavlova, M. I., \& Groot, W. N. J. (2010). Attitudes towards patient payments in Ukraine: Is there a place for official patient charges? Zeszyty Naukowe Ochrony Zdrowia. Zdrowie Publiczne i Zarzadzanie [Public Health and Management], 8(1), 69-78.

Gulácsi, L., Boncz, I., Baji, P., \& Péntek, M. (2012). Költségszámítás [Costing]. In L. Gulácsi (Ed.), Egészség-gazdaságtan és technológiaelemzés. [Health Economics and Technology Assessment] (pp. 135-190). Budapest: Medicina Könyvkiadó Zrt.

Günther, O. H., Kürstein, B., Riedel-Heller, S. G., \& König, H. H. (2010). The role of monetary and nonmonetary incentives on the choice of practice establishment: a stated preference study of young physicians in Germany. Health Services Research, 45(1), 212229.

Gyrd-Hansen, D., \& Kjaer, T. (2012). Disentangling WTP per QALY data: Different analytical approaches, different answers. Health Economics, 21(3), 222-237.

Hammerschmidt, T., Zeitler, H. P., \& Leidl, R. (2004). A utility-theoretic approach to the aggregation of willingness to pay measured in decomposed scenarios: development and empirical test. Health Economics, 13(4), 345-361. 


\section{References}

Hancock-Howard, R. L., Ungar, W. J., Marshall, D., Einarson, A., \& Koren, G. (2012). Public preferences for counseling regarding antidepressant use during pregnancy: a discrete choice experiment. Birth Defects Research. Part A, Clinical and Molecular Teratology, 94(7), 532-539.

Hanemann, M., Loomis, J., \& Kanninen, B. (1991). Statistical efficiency of double-bounded dichotomous choice contingent valuation. American Journal of Agricultural Economics, 73(4), 1255-1263.

Hanemann, W. M. (1991). Willingness to pay and willingness to accept: How much can they differ? The American Economic Review, 81(3), 635-647.

Hanley, N., MacMillan, D., Wright, R. E., Bullock, C., Simpson, I., Parsisson, D., \& Crabtree, B. (1998). Contingent valuation versus choice experiments: estimating the benefits of environmentally sensitive areas in Scotland. Journal of Agricultural Economics, 49(1), 115.

Hanley, N., Ryan, M., \& Wright, R. (2003). Estimating the monetary value of health care: Lessons from environmental economics. Health Economics, 12(1), 3-16.

Hansen, K. S., Pedrazzoli, D., Mbonye, A., Clarke, S., Cundill, B., Magnussen, P., \& Yeung, S. (2013). Willingness-to-pay for a rapid malaria diagnostic test and artemisinin-based combination therapy from private drug shops in Mukono district, Uganda. Health Policy and Planning, 28(2), 185-196.

Hanson, K., McPake, B., Nakamba, P., \& Archard, L. (2005). Preferences for hospital quality in Zambia: Results from a discrete choice experiment. Health Economics, 14(7), 687701.

Harder, D. S., Jian-jun, J., Francisco, H. A., \& Sajise, A. J. U. (2006). WTP Calibration and Bias Transfer: a Stochastic Frontier Approach. Paper presented at the Third World Congress of Environmental and Resource Economists, Kyoto, Japan.

Harrison, G. W., \& Rutström, E. E. (2008). Experimental Evidence on the Existence of Hypothetical Bias in Value Elicitation Methods. In R. P. Charles \& L. S. Vernon (Eds.), Handbook of Experimental Economics Results (Vol. Volume 1, pp. 752-767): Elsevier.

Havet, N., Morelle, M., Remonnay, R., \& Carrere, M. O. (2012). Cancer patients' willingness to pay for blood transfusions at home: Results from a contingent valuation study in a French cancer network. European Juornal of Health Economics, 13(3), 289-300. 
Hensher, D. A. (2010). Hypothetical bias, choice experiments and willingness to pay. Transportation Research. Part B: Methodological, 44(6), 735-752.

Hicks, J. R. (1943). The four consumer's surpluses. The Review of Economic Studies, 11(1), $31-41$.

Hjelmgren, J., \& Anell, A. (2007). Population preferences and choice of primary care models: A discrete choice experiment in Sweden. Health Policy, 83(2-3), 314-322.

Ho, J.-J., Liu, J.-T., \& Wang, J.-D. (2005). Stated preferences for the removal of physical pain resulting from permanently disabling occupational injuries: A contingent valuation study of Taiwan. Accident Analysis \& Prevention, 37(3), 537-548.

Hodgkins, P., Swinburn, P., Solomon, D., Yen, L., Dewilde, S., \& Lloyd, A. (2012). Patient preferences for first-line oral treatment for mild-to-moderate ulcerative colitis: a discretechoice experiment. Patient, 5(1), 33-44.

Hoehn, J. P., \& Randall, A. (1987). A satisfactory benefit cost indicator from contingent valuation. Journal of Environmental Economics and Management, 14(3), 226-247.

Hofler, R. A., \& List, J. A. (2004). Valuation on the frontier: Calibrating actual and hypothetical statements of value. American Journal of Agricultural Economics, 86(1), 213-221.

Hosmer, D. W., Lemeshow, S., \& May, S. (2008). Applied Survival Analysis: John Wiley \& Sons, Inc.

Huber, J., \& Zwerina, K. (1996). The importance of utility balance in efficient choice designs. Journal of Marketing Research, 33, 307-317.

Jakab, M., \& Kutzin, J. (2009). Improving financial protection in Kyrgyzstan through reducing informal payments. Evidence from 2001-06. (Policy research paper no. 57) Geneva: World Health Organization; 2009. Retrieved from: http://www.hpac.kg/images/pdf/PRP57.eng.pdf.

Jendle, J., Torffvit, O., Ridderstrale, M., Ericsson, A., Nilsen, B., \& Bogelund, M. (2012). Willingness to pay for diabetes drug therapy in type 2 diabetes patients: Based on LEAD clinical programme results. Journal of Medical Economics, 15 Suppl 2, 1-5.

Jenkins, S. P. (2005). Survival Analysis. Retrieved from http://michau.nazwa.pl/ aska/uploads/Studenci/mag7_1.pdf 


\section{References}

Johnson, R., Huber, J., \& Bacon, L. (2003). Adaptive Choice Based Conjoint Analysis. Sawtooth Software. Research Paper Series. Retrieved from http://www.sawtoothsoftware.com/download/techpap/acbc.pdf

Karamyshev, D., Vashev, Î., Nemchenko, A., \& Volos, B. (2006). State Policy of Public Health Service Financing in Ukraine. (in Ukrainian). Har RI NADU Derzhavne budivnytstvo, 1.

Kartman, B., Stålhammar, N. O., \& Johannesson, M. (1996). Valuation of health changes with the contingent valuation method: a test of scope and question order effects. Health Economics, 5(6), 531-541.

Kerr, E. A., Hays, R. D., Mitchinson, A., Lee, M., \& Siu, A. L. (1999). The influence of gatekeeping and utilization review on patient satisfaction. Journal of General Internal Medicine, 14(5), 287-296.

Kjaer, T., Bech, M., Kronborg, C., \& Morkbak, M. R. (2012). Public preferences for establishing nephrology facilities in Greenland: Estimating willingness-to-pay using a discrete choice experiment. European Journal of Health Economics, 14(5), 739-748.

Klose, T. (1999). The contingent valuation method in health care. Health Policy, 47(2), 97123.

Koppel, A., Kahur, K., Habicht, T., Saar, P., Habicht, J., \& van Ginneken, E. (2008). Estonia: Health System Review. Health Systems in Transition, 10(1), 1-230.

Krupnick, A. J., \& Cropper, M. L. (1992). The effect of information on health risk valuations. Journal of Risk and Uncertainty, 5(1), 29-48.

Lancsar, E., \& Louviere, J. (2008). Conducting discrete choice experiments to inform healthcare decision making. Pharmacoeconomics, 26(8), 661-677.

Lancsar, E., Louviere, J., \& Flynn, T. (2007). Several methods to investigate relative attribute impact in stated preference experiments. Social Science \& Medicine, 64(8), 1738-1753.

Lancsar, E., \& Savage, E. (2004). Deriving welfare measures from discrete choice experiments: Inconsistency between current methods and random utility and welfare theory. Health Economics, 13(9), 901-907. 
Lang, H. C., Chang, K., \& Ying, Y. H. (2012). Quality of life, treatments, and patients' willingness to pay for a complete remission of cervical cancer in Taiwan. Health Economics, 21(10), 1217-1233.

Law of Ukraine (2011). About regime of reforming the health care system in the regions of Vinnytsia, Dnipropetrovsk, Donetsk, and in the Kyiv city. (in Ukrainian). Retrieved from http://zakon2.rada.gov.ua/laws/show/3612-17

Lekhan, V., Rudiy, V., \& Nolte, E. (2004). Ukraine. Health care systems in transition, 6(8), $1-128$.

Lekhan, V., Rudiy, V., \& Richardson, E. (2010a). Ukraine: Health system review. Health Systems in Transition, 12(8), 1-183.

Lekhan, V., Slabkyj, G., \& Shevchenko, M. (2010b). Health care system strategy development: Ukrainian measuring. Ukraina. (in Ukrainian). Zdorovja naciji 1, 5-23.

Leon, M. (2003). Perceptions of health care quality in Central America. International Journal for Quality in Health Care, 15(1), 67-071.

Lever, L. (2013). New co-payment for hospital patients in Romania is in place, Romaniainsider.com. Retrieved from http://www.romania-insider.com/new-co-payment-forhospital-patients-in-romania-is-in-place/78754/

Lewis, M. (2000). Who is Paying for Health Care in Eastern Europe and Central Asia? Washington, D.C.: World Bank.

Lewis, M. (2002). Informal health payments in central and eastern Europe and the former Soviet Union: issues, trends and policy implications. In E. Mossialos, A. Dixon, J. Figueras \& J. Kutzin (Eds.), Funding health care: options for Europe (pp. 184-206). Buckingham: Open University Press.

Lewis, M. (2006). Governance and Corruption in Public Health Care Systems. (Working paper No. 78). Center for Global Development.

Lewis, M. (2007). Informal Payments And The Financing Of Health Care In Developing And Transition Countries. Health Affairs, 26(4), 984-997.

Liljas, B., \& Blumenschein, K. (2000). On hypothetical bias and calibration in cost-benefit studies. Health Policy, 52(1), 53-70. 


\section{References}

Linden, M., Gothe, H., \& Ormel, J. (2003). Pathways to care and psychological problems of general practice patients in a "gate keeper" and an" open access" health care system. Social Psychiatry and Psychiatric Epidemiology, 38(12), 690-697.

List, J., \& Gallet, C. (2001). What experimental protocol influence disparities between actual and hypothetical stated values? Environmental and Resource Economics, 20(3), 241-254.

Litvak, A., Pogorilyj, V., \& Tyshchuk, M. (2001). Shade economy and future of the medical care in Ukraine. (in Ukrainian). Odessa: Vyd.TES.

Liu, Y., Rao, K., \& Hsiao, W. C. (2011). Medical expenditure and rural impoverishment in China. Journal of Health, Population and Nutrition (JHPN), 21(3), 216-222.

Loh, C. P., \& Shapiro, A. (2013). Willingness to pay for home- and community-based services for seniors in Florida. Home Health Care Services Quarterly, 32(1), 17-34.

Loomis, J. (2011). What's to know about hypothetical bias in stated preference valuation studies? Journal of Economic Surveys, 25(2), 363-370.

Loomis, J., Gonzalez-Caban, A., \& Gregory, R. (1994). Do reminders of substitutes and budget constraints influence contingent valuation estimates? Land Economics, 70(4), 499-506.

Louviere, J., Street, D., Burgess, L., Wasi, N., Islam, T., \& Marley, A. A. (2008). Modeling the choices of individual decision-makers by combining efficient choice experiment designs with extra preference information. Journal of Choice Modelling, 1(1), 128-163.

Louviere, J. J. (2006). What you don't know might hurt you: some unresolved issues in the design and analysis of discrete choice experiments. Environmental and Resource Economics, 34(1), 173-188.

Louviere, J. J., Flynn, T. N., \& Carson, R. T. (2010). Discrete choice experiments are not conjoint analysis. Journal of Choice Modelling, 3(3), 57-72.

Louviere, J. J., Hensher, D. A., \& Swait, J. D. (2000). Stated choice methods: analysis and applications. Cambridge University Press.

Louviere, J. J., \& Lancsar, E. (2009). Choice experiments in health: the good, the bad, the ugly and toward a brighter future. Health Economics, Policy and Law, 4(4), 527-546. 
Lunander, A. (1998). Inducing incentives to understate and to overstate willingness to pay within the open-ended and the dichotomous-choice elicitation formats: An experimental study. Journal of Environmental Economics and Management, 35(1), 88-102.

Lynn, F. A., Crealey, G. E., Alderdice, F. A., \& McElnay, J. C. (2013). Preferences for a third-trimester ultrasound scan in a low-risk obstetric population: A discrete choice experiment. Health Expectations, Doi: 10.1111/hex.12062. [Epub ahead of print].

Manning, W. G., Newhouse, J. P., Duan, N., Keeler, E. B., Leibowitz, A., Marquis, M. S., \& Zwanziger, J. (1988). Health Insurance and the Demand for Medical Care: Evidence from a Randomized Experiment. Santa Monica, CA: RAND Corporation.

Mansfield, C. (1999). Despairing over disparities: Explaining the difference between willingness to pay and willingness to accept. Environmental and Resource Economics, 13(2), 219-234.

Marti, J. (2012). Assessing preferences for improved smoking cessation medications: A discrete choice experiment. European Journal of Health Economics, 13(5), 533-548.

Martín-Fernández, J., del Cura-González, M. I., Gómez-Gascón, T., Oliva-Moreno, J., Domínguez-Bidagor, J., Beamud-Lagos, M., \& Pérez-Rivas, F. J. (2010a). Differences between willingness to pay and willingness to accept for visits by a family physician: A contingent valuation study. BMC Public Health, 10(1), 236, DOI: 10.1186/1471-245810-236.

Martín-Fernández, J., Gómez-Gascón, T., Oliva-Moreno, J., del Cura-González, M. I., Domínguez-Bidagor, J., Beamud-Lagos, M., \& Sanz-Cuesta, T. (2010b). Perception of the economic value of primary care services: A willingness to pay study. Health Policy, 94(3), 266-272.

Mataria, A., Donaldson, C., Luchini, S., \& Moatti, J. P. (2004). A stated preference approach to assessing health care-quality improvements in Palestine: From theoretical validity to policy implications. Journal of Health Economics, 23(6), 1285-1311.

Mataria, A., Luchini, S., Daoud, Y., \& Moatti, J. P. (2007). Demand assessment and priceelasticity estimation of quality-improved primary health care in Palestine: a contribution from the contingent valuation method. Health Economics, 16(10), 1051-1068. 


\section{References}

Mattsson, L. A., Ericsson, A., Bogelund, M., \& Maamari, R. (2013). Women's preferences toward attributes of local estrogen therapy for the treatment of vaginal atrophy. Maturitas, Doi: 10.1016/j.maturitas.2012.12.004. [Epub ahead of print].

McPake, B. (1993). User charges for health services in developing countries: A review of the economic literature. Social Science \& Medicine, 36(11), 1397-1405.

Mele, N. L. (2008). Conjoint analysis: Using a market-based research model for healthcare decision making. Nursing Research, 57(3), 220-224.

Ministry of Health of Ukraine. (2010). Project Concept of managing quality in health care for the period till 2020. (in Ukrainian). Retrieved from http://www.moz.gov.ua/ ua/portal/Pro_20110707_0.html.

Mitani, Y., \& Flores, N. E. (2010). Hypothetical bias reconsidered: payment and provision uncertainties in a threshold provision mechanism. Paper presented at the World Congress on Environmental and Resource Economics, Montreal Canada.

Mitchell, R. C., \& Carson, R. T. (1989). Using surveys to value public goods: The contingent valuation method. Washington, D.C: Resources for the Future.

Mitenbergs, U., Taube, M., Misins, J., Mikitis, E., Martinsons, A., Rurane, A., \& Quentin, W. (2012). Latvia: Health system review. Health Systems in Transition, 14(8), 1-191.

Mocan, H. N., Tekin, E., \& Zax, J. S. (2004). The Demand for Medical Care in Urban China. World Development, 32(2), 289-304.

Mogas, J., Riera, P., \& Brey, R. (2009). Combining contingent valuation and choice experiments. A forestry application in Spain. Environmental and Resource Economics, 43(4), 535-551.

Moia, M., Mantovani, L. G., Carpenedo, M., Scalone, L., Monzini, M. S., Cesana, G., \& Mannucci, P. M. (2013). Patient preferences and willingness to pay for different options of anticoagulant therapy. Internal and Emergency Medicine, 8(3), 237-243.

Mosseveld van, C., Kawiorska, D., \& De Norre, B. (2008). Health expenditure, 2003-2005. Eurostat: Data in focus. Retrieved from: http://epp.eurostat.ec.europa.eu /cache/ITY_OFFPUB/KS-QA-08-026/EN/KS-QA-08-026-EN.PDF.

Murauskiene, L., Janoniene, R., Veniute, M., van Ginneken, E., \& Karanikolos, M. (2013). Lithuania: Health system review. Health Systems in Transition, 15(2): 1-150. 
Murauskiene, L., Pavlova, M., Veniute, M., \& Groot, W. (2012). Towards a more comprehensive view on patient payments in Lithuania: New findings from a population survey. Society and Economy, 34(2), 241-251.

Murauskiene, L., Veniute, M, \& Pavlova, M. (2010). The Opinions of the Key Health Care Stakeholders towards Patient Payments in Lithuania. Zeszytu Naukowe Ochrony Zdrowia. Zdrowie Publiczne i Zarzadzanie [Public Health and Management], 8(1), 62-68.

Murphy, J., Allen, P. G., Stevens, T., \& Weatherhead, D. (2005). A meta-analysis of hypothetical bias in stated preference valuation. Environmental and Resource Economics, 30(3), 313-325.

Naik-Panvelkar, P., Armour, C., Rose, J., \& Saini, B. (2012). Patients' value of asthma services in Australian pharmacies: the way ahead for asthma care. Journal of Asthma, 49(3), 310-316.

National Health Insurance Fund of Bulgaria. (2011). Договор № РД-НС-01-3 от 30 декември 2011 г. за приемане на обеми и цени на медицинската помощ [Contract \#РД-НС-01-3 from December 30, 2011 adopting the volumes and prices of medical care]. Retrieved from http://www.nhif.bg/c/document_library/get_file?p_l $\underline{\mathrm{id}=58538 \& \text { folderId }=215182 \& \text { name=DLFE-4890.doc }}$

National Health Insurance Fund of Romania. (2011). Ordinul ministrului sanatatii si a presedintelui Casei Nationale de Asigurari de Sanatate nr. 1723/950/2011 pentru aprobarea Normelor metodologice de aplicare in anul 2012 a Contractului cadru [Order of the Minister of Health and President of the National Health Insurance no. 1723/950/2011 Methodological Norms for the application in 2012 of the framework agreement]. Retrived from http://www.cnas.ro/legislatie/noutati-legislative/ordinulministrului-sanatatii-si-a-presedintelui-casei-nationale-de-asigurari-de-sanatate-nr-1723$\underline{950-2011}$

National Statistical Institute of Bulgaria. (2012). Inflation and Consumer Price Indices Table data Retrieved from http://www.nsi.bg/otrasalen.php?otr=47, Accessed: August 21, 2012.

Neuman, S., \& Neuman, E. (2008). What women want when they need medical treatment? Retrieved from http://www.voxeu.org/article/are-hospitals-serving-patients-preferences 


\section{References}

Newhouse, J. P., \& Group, I. E. (1993). Free for All? Lessons from the RAND Health Experiment. Cambridge, Mass: Harvard University Press.

Nieboer, A. P., Koolman, X., \& Stolk, E. A. (2010). Preferences for long-term care services: willingness to pay estimates derived from a discrete choice experiment. Social Science \& Medicine, 70(9), 1317-1325.

Nolan, B. (1993). Economic incentives, health status and health services utilisation. Journal of Health Economics, 12, 151-169.

O'Brien, B., \& Gafni, A. (1996). When do the" dollars" make sense? Toward a conceptual framework for contingent valuation studies in health care. Medical Decision Making, 16(3), 288-299.

OECD. (2011). Health at a Glance: OECD Indicators: OECD. OECD. Retrieved from http://www.oecd.org/els/health-systems/49105858.pdf.

OECD. (2012a). Health at a Glance: OECD indicators: Europe 2012. OECD. Retrieved from http://www.oecd.org/els/health-systems/HealthAtAGlanceEurope2012.pdf.

OECD. (2012b). National CPI weights Retrieved August21, 2012, from http://stats.oecd.org/Index.aspx?DataSetCode=MEI_CPI_WEIGHTS\#

Olaru, D., Smith, B., \& Wang, J. (2011). Optimal discrete choice experimental designs using genetic algorithms. Paper presented at the International Choice Modelling Conference, Leeds, UK. http://www.icmconference.org.uk/index.php/icmc/ICMC2011/paper/view/390

Olsen, J. A., \& Smith, R. D. (2001). Theory versus practice: a review of 'willingness-topay'in health and health care. Health Economics, 10(1), 39-52.

Orea, L., \& Kumbhakar, S. C. (2004). Efficiency measurement using a latent class stochastic frontier model. Empirical Economics, 29(1), 169-183.

Pavlova, M., Groot, W., \& van Merode, F. (2000). Appraising the financial reform in Bulgarian public health care sector: the health insurance act of 1998. Health Policy, 53(3), 185-199.

Pavlova, M., Groot, W., \& van Merode, G. (2003). The importance of quality, access and price to health care consumers in Bulgaria: A self-explicated approach. The International Journal of Health Planning and Management, 18(4), 343-361. 
Pavlova, M., Groot, W., \& Van Merode, G. (2004). Willingness and ability of Bulgarian consumers to pay for improved public health care services. Applied Economics, 36(10), 1117-1130.

Pavlova, M., Groot, W., van Merode, G., \& Project Team. (2011). Is there a place for an increased reliance on patient charges in central and eastern european countries. Scripta Scientifica Medica, 43(7), 137-141.

Pavlova, M., Hendrix, M., Nouwens, E., Nijhuis, J., \& van Merode, G. (2009). The choice of obstetric care by low-risk pregnant women in the Netherlands: Implications for policy and management. Health Policy, 93(1), 27-34.

Pavlova, M., Tambor, M., Stepurko, T., Merode, G. G., \& Groot, W. (2012). Assessment of patient payment policy in CEE countries: From a conceptual framework to policy indicators. Society and Economy, 34(2), 193-220.

Pavlova, M., Tambor, M., van Merode, G., \& Groot, W. (2010). Are patient charges an effective policy tool? Review of theoretical and empirical evidence. Zeszyty Naukowe Ochrony Zdrowia. Zdrowie Publiczne i Zarzadzanie [Public Health and Management], 8(1), 29-36.

Pol van der, M., \& Cairns, J. (2008). Comparison of two methods of eliciting time preference for future health states. Social Science \& Medicine, 67(5), 883-889.

Pol van der, M., Shiell, A., Au, F., Johnston, D., \& Tough, S. (2008). Convergent validity between a discrete choice experiment and a direct, open-ended method: Comparison of preferred attribute levels and willingness to pay estimates. Social Science \& Medicine, 67(12), 2043-2050.

Polachek, S. W., \& Yoon, B. J. (1987). A two-tiered earnings frontier estimation of employer and employee information in the labor market. The Review of Economics and Statistics, 69(2), 296-302.

Polachek, S. W., \& Yoon, B. J. (1996). Panel estimates of a two-tiered earnings frontier. Journal of Applied Econometrics, 11(2), 169-178.

Popovich, L., Potapchik, E., Shishkin, S., Richardson, E., Vacroux, A., \& Mathivet, B. (2011). Russain Federation: Health system review. Health Systems in Transition, 13(7), $1-190$. 


\section{References}

Prosser, L. A., Payne, K., Rusinak, D., Shi, P., \& Messonnier, M. (2013). Using a discrete choice experiment to elicit time trade-off and willingness-to-pay amounts for influenza health-related Quality of Life at different ages. Pharmacoeconomics, 31(4), 305-315.

Radu, O. (2010). By summer, new law on additional mandatory health insurance in Bulgaria will be passed. 1asig.ro. Retrieved from http://insurance.1asig.ro/By-summer-new-lawon-additional-mandatory-health-insurance-in-Bulgaria-will-be-passed-article-2,3,117$\underline{30114 . h t m}$

Rechel, B., \& McKee, M. (2009). Health reform in central and eastern Europe and the former Soviet Union. The Lancet, 374(9696), 1186-1195.

Regier, D. A., Diorio, C., Ethier, M. C., Alli, A., Alexander, S., Boydell, K. M., Gassas, A., Taylor, J., Kellow, C., Mills, D., \& Sung, L. (2012). Discrete choice experiment to evaluate factors that influence preferences for antibiotic prophylaxis in pediatric oncology. PLoS One, 7(10), e47470. Doi:10.1371/journal.pone.0047470.

Rischatsch, M., \& Zweifel, P. (2012). What do physicians dislike about managed care? Evidence from a choice experiment. European Journal of Health Economics, 14(4), 601613.

Rogers, A., Kennedy, A., Nelson, E., \& Robinson, A. (2004). Patients' experiences of an open access follow up arrangement in managing inflammatory bowel disease. Quality and Safety in Health Care, 13(5), 374-378.

Ros, C. C., Groenewegen, P. P., \& Delnoij, D. M. J. (2000). All rights reserved, or can we just copy? Cost sharing arrangements and characteristics of health care systems. Health Policy, 52(1), 1-13.

Rovira, J., Mompo, C., Wildt, K. D., Schneider, M., \& Blasco, I. (1998). Cost-sharing in European union member states a system oriented framework. In R. Leidl (Ed.), Health care and its financing in the single European market. Amsterdam: IOS Press.

Rückert, I.-M., Böcken, J., \& Mielck, A. (2008). Are German patients burdened by the practice charge for physician visits ('Praxisgebuehr')? A cross sectional analysis of socioeconomic and health related factors. BMC Health Services Research, 8(1), 232.

Russell, S. (1996). Ability to pay for health care: concepts and evidence. Health Policy and Planning, 11(3), 219-237. 
Ryan, M. (1999). Using conjoint analysis to take account of patient preferences and go beyond health outcomes: an application to in vitro fertilisation. Social Science \& Medicine, 48(4), 535-546.

Ryan, M. (2004a). A comparison of stated preference methods for estimating monetary values. Health Economics, 13(3), 291-296.

Ryan, M. (2004b). Deriving welfare measures in discrete choice experiments: a comment to Lancsar and Savage (1). Health Economics, 13(9), 909-912.

Ryan, M. (2004c). Discrete choice experiments in health care: NICE should consider using them for patient centred evaluations of technologies. British Medical Journal, 328(7436), 360, Doi: 10.1136/bmj.328.7436.360.

Ryan, M., Bate, A., Eastmond, C. J., \& Ludbrook, A. (2001a). Use of discrete choice experiments to elicit preferences. Quality in Health Care, 10(Suppl 1), i55-60.

Ryan, M., \& Farrar, S. (2000). Using conjoint analysis to elicit preferences for health care. British Medical Journal, 320(7248), 1530, Doi: 10.1136/bmj.320.7248.1530.

Ryan, M., \& Gerard, K. (2003). Using discrete choice experiments to value health care programmes: current practice and future research reflections. Applied Health Economics and Health Policy, 2(1), 55-64.

Ryan, M., Scott, D., Reeves, C., Bate, A., Van Teijlingen, E., Russell, E., Napper, M., \&Robb, C. (2001b). Eliciting public preferences for healthcare: A systematic review of techniques. Health Technology Assessment (Winchester, England), 5(5), 1-186.

Ryan, M., Scott, D. A., \& Donaldson, C. (2004). Valuing health care using willingness to pay: a comparison of the payment card and dichotomous choice methods. Journal of Health Economics, 23(2), 237-258.

Ryan, M., \& Skåtun, D. (2004). Modelling non-demanders in choice experiments. Health Economics, 13(4), 397-402.

Ryan, M., \& Watson, V. (2009). Comparing welfare estimates from payment card contingent valuation and discrete choice experiments. Health Economics, 18(4), 389-401.

Ryan, M., \& Wordsworth, S. (2000). Sensitivity of willingness to pay estimates to the level of attributes in discrete choice experiments. Scottish Journal of Political Economy, 47(5), 504-524. 


\section{References}

Sadique, M. Z., Devlin, N., Edmunds, W. J., \& Parkin, D. (2013). The effect of perceived risks on the demand for vaccination: results from a discrete choice experiment. PLoS One, 8(2), e54149, Doi: 10.1371/journal.pone.0054149.

Sagan, A., Panteli, D., Borkowski, W., Dmowski, M., Domański, F., Czyżewski, M., Goryński, P., Karpacka, D., Kiersztyn, E., Kowalska, I., Księżak, M., Kuszewski, K., Leśniewska, A., Lipska, I., Maciąg, R., Madowicz, J., Mądra, A., Marek, M., Mokrzycka, A., Poznański, D., Sobczak, A., Sowada, C., Świderek, M., Terka, A., Trzeciak, P., Wiktorzak, K., Włodarczyk, C., Wojtyniak, B., Wrześniewska-Wal, I., Zelwiańska, D. \& Busse, R. (2011). Poland: Health system review. Health Systems in Transition, 13(8), 1193.

Samuelson, P. A. (1954). The Pure Theory of Public Expenditure. The Review of Economics and Statistics, 36(4), 387-389.

Santos Silva, J. (2004). Deriving welfare measures in discrete choice experiments: a comment to Lancsar and Savage (2). Health Economics, 13(9), 913-918.

Saulo, E., Forsberg, B., Premji, Z., Montgomery, S., \& Björkman, A. (2008). Willingness and ability to pay for artemisinin-based combination therapy in rural Tanzania. Malaria Journal, 7(1), 227, Doi: 10.1186/1475-2875-7-227.

SCB Statistics Sweden. (2010). Income distribution survey 2008. Retrieved from http://www.scb.se/statistik/HE/HE0103/2008A02D/HE0103_2008A02D_SM_HE21SM1 001.pdf

Schmitt, J., Kirch, W., \& Meurer, M. (2009). Effects of the introduction of the German "Praxisgebuhr" on outpatient care and treatment of patients with atopic eczema. Journal der Deutschen Dermatologischen Gesellschaft [Journal of the German Society of Dermatology], 7(10), 879-886.

Schwarz, G. (1978). Estimating the dimension of a model. The Annals of Statistics, 6(2), 461464.

Schwenkglenks, M., Preiswerk, G., Lehner, R., Weber, F., \& Szucs, T. D. (2006). Economic efficiency of gate-keeping compared with fee for service plans: A Swiss example. The Journal of Epidemiology \& Community Health, 60(1), 24-30.

Sen, A. (1992). Inequality reexamined. Clarendon Press. 
Sepehri, A., \& Chernomas, R. (2001). Are user charges efficiency- and equity-enhancing? A critical review of economic literature with particular reference to experience from developing countries. Journal of International Development, 13(2), 183-209.

Shackley, P., \& Donaldson, C. (2002). Should we use willingness to pay to elicit community preferences for health care?: New evidence from using a 'marginal' approach. Journal of Health Economics, 21(6), 971-991.

Shafie, A. A., \& Hassali, M. A. (2013). Willingness to pay for voluntary community-based health insurance: Findings from an exploratory study in the state of Penang, Malaysia. Social Science \& Medicine, 96, 272-276.

Skriabikova, O., Pavlova, M., \& Groot, W. (2010). Empirical Models of Demand for OutPatient Physician Services and Their Relevance to the Assessment of Patient Payment Policies: A Critical Review of the Literature. International Journal of Environmental Research and Public Health, 7(6), 2708-2725.

Slothuus Skjoldborg, U., \& Gyrd-Hansen, D. (2003). Conjoint analysis. The cost variable: an Achilles' heel? Health Economics, 12(6), 479-491.

Smith, R. D., \& Sach, T. H. (2010). Contingent valuation: What needs to be done? Health Economics, Policy, and Law, 5(Pt 1), 91-111.

Sogaard, R., Lindholt, J., \& Gyrd-Hansen, D. (2012). Insensitivity to scope in contingent valuation studies: reason for dismissal of valuations? Applied Health Economics \& Health Policy, 10(6), 397-405.

Starfield, B. (1992). Primary care: concept, evaluation, and policy: Oxford University Press, USA.

State Statistical Service of Ukraine. (2010a). Average salary by economic activity types in 2009. Retrieved from http://www.ukrstat.gov.ua/operativ/operativ2009/gdn/Zarp_ek_m lzpm2009_u.htm

State Statistical Service of Ukraine. (2010b). Self-appraisal of health status and level of access to certain types of health care services in 2009. (in Ukrainian). Retrieved from http://ukrstat.org/uk/operativ/operativ2010/gdn/sns/sns_2009.htm

Statistics Lithuania. (2012). Consumer price indeces. Retrieved from http://www.stat.gov.lt /en/pages/view/?id=1370 


\section{References}

Stepurko, T., Pavlova, M., Gryga, I., \& Groot, W. (2010). Empirical studies on informal patient payments for health care services: a systematic and critical review of research methods and instruments. BMC Health Services Research, 10(1), 273, Doi: 10.1186/1472-6963-10-273.

Stepurko, T., Pavlova, M., Gryga, I., \& Groot, W. (2011). Informal patient payments and public attitudes towards these payments: Evidence from six CEE countries. Scripta Scientifica Medica, 43(7), 143-146.

Stepurko, T., Pavlova, M., Gryga, I., Groot, W.(2013). Informal payments for health care services-Corruption or gratitude? A study on public attitudes, perceptions and opinions in six Central and Eastern European countries. Communist and Post-Communist Studies, 46(4), 419-431. Doi: 10.1016/j.postcomstud.2013.08.004.

Stepurko, T., Pavlova, M., Gryga, I., Murauskiene, L., \& Groot, W. (2013). Informal payments for health care services: the case of post-Soviet republics Lithuania and Ukraine. In J. Morris \& A. Polese (Eds.), The Persistence of Informal Economic Practices in Post-Socialist Societies: Palgrave Macmillan.

Stepurko, T., Pavlova, M., Levenets, O., Gryga, I., \& Groot, W. (2012). Informal patient payments in maternity hospitals in Kiev, Ukraine. The International Journal of Health Planning and Management, 28(2), e169-187.

Strazzera, E., Scarpa, R., Calia, P., Garrod, G. D., \& Willis, K. G. (2003). Modelling zero values and protest responses in contingent valuation surveys. Applied Economics, 35(2), 133-138.

Street, D. J., Burgess, L., \& Louviere, J. J. (2005). Quick and easy choice sets: constructing optimal and nearly optimal stated choice experiments. International Journal of Research in Marketing, 22(4), 459-470.

Survey: Almost half of the Ukrainians regret that USSR collapsed [in Ukrinian]. (2011). Novynar. Retrieved from http://novynar.com.ua/politics/150718

Szende, A., \& Culyer, A. J. (2006). The inequity of informal payments for health care: the case of Hungary. Health Policy, 75(3), 262-271.

Tambor, M., Pavlova, M., Woch, P., \& Groot, W. (2011). Diversity and dynamics of patient cost-sharing for physicians' and hospital services in the 27 European Union countries. The European Journal of Public Health, 21(5), 585-590. 
Taylor, S. J., \& Armour, C. L. (2002). Acceptability of willingness to pay techniques to consumers. Health Expectations, 5(4), 341-356.

Telser, H., \& Zweifel, P. (2002). Measuring willingness-to-pay for risk reduction: an application of conjoint analysis. Health Economics, 11(2), 129-139.

Telser, H., \& Zweifel, P. (2007). Validity of discrete-choice experiments evidence for health risk reduction. Applied Economics, 39(1), 69-78.

Thaler, R. (1980). Toward a positive theory of consumer choice. Journal of Economic Behavior \& Organization, 1(1), 39-60.

Thompson, R., \& Witter, S. (2000). Informal payments in transitional economies: implications for health sector reform. The International Journal of Health Planning and Management, 15(3), 1099-1751.

Thomson, S., Võrk, A., Habicht, T., Rooväli, L., Evetovits, T., \& Habicht, J. (2010). Responding to the challenge of financial sustainability in Estonia's health system. WHO Regional Office for Europe.

Tomini, S., Groot, W., \& Pavlova, M. (2012a). Paying informally in the Albanian health care sector: a two-tiered stochastic frontier model. The European Journal of Health Economics, 13(6), 777-788.

Tomini, S. M., Packard, T. G., \& Tomini, F. (2012b). Catastrophic and impoverishing effects of out-of-pocket payments for health care in Albania: evidence from Albania Living Standards Measurement Surveys 2002, 2005 and 2008. Health Policy \& Planning, 28(4), 419-428.

Tsionas, E. G. (2012). Maximum likelihood estimation of stochastic frontier models by the Fourier transform. Journal of Econometrics, 170(1), 234-248.

TSN. (2011). All Ukrainian top officials seek treatment abroad. (in Ukrainian). TSN. Retrieved from http://tsn.ua/ukrayina/vischi-ukrayinski-chinovniki-pogolovno$\underline{\text { likuyutsya-za-kordonom.html }}$

Tverdokhlib, O., \& Horshkov, Y. V. (2011). Classification of medical institutions for the tasks of health insurcance. Medical Informatics and Engineering, 2, 64-67.

Ukrainian Helsinki Human Rights Union. 2012. Annual human rights report Ukraine. Human rights in Ukraine 2011. Retrieved from http://helsinki.org.ua/index.php?id=1332408556 


\section{References}

Ukraïna. (1996). Constitution of Ukraine: Ukrainian Legal Foundation.

Vian, T., Gryboskb, K., Sinoimeric, Z., \& Halld, R. (2006). Informal payments in government health facilities in Albania: Results of a qualitative study. Social Science \& Medicine, 62(4), 877-887.

Vick, S., \& Scott, A. (1998). Agency in health care. Examining patients' preferences for attributes of the doctor-patient relationship. Journal of Health Economics, 17(5), 587605.

Vilnius Teritorial Insurance Fund. (2012). Ambulatorinių specializuotu asmens sveikatos priežiūros paslaugų kainos [Specialized outpatient personal health care service prices]. Retrieved from http://www.vilniaustlk.lt/index.php?id=45

Vladescu, C., Scintee, G., \& Olsavszsky, V. (2008). Romania: Health system Review. Health Systems in Transition, 10(3), 1-172.

Vončina, L., Strizrep, T., Bagat, M., Pezelj-Duliba, D., Pavić, N., \& Polašek, O. (2012). Croatian 2008-2010 health insurance reform: hard choices toward financial sustainability and efficiency. Croatian Medical Journal, 53(1), 66-76.

Voorde van de, C., van Doorslaer, E., \& Schokkaert, E. (2001). Effects of cost sharing on physician utilization under favourable conditions for supplier-induced demand. Health Economics, 10(5), 457-471.

Vynogradov, O. (2007). State and the problems of the financial resources of health care in Ukraine. (in Ukrainian). Economica ta Derzhava 12, 25-29.

Wagstaff, A., \& Van Doorslaer, E. (1992). Equity in the finance of health care: some international comparisons. Journal of Health Economics, 11(4), 361-387.

Walraven, G. (1996). Willingness to pay for district hospital services in rural Tanzania. Health Policy \& Planning, 11(4), 428-437.

Wang, J., \& Hong, S. H. (2012). Contingent valuation and pharmacists' acceptable levels of compensation for medication therapy management services. Research in Social and Administrative Pharmacy, Doi: 10.1016/j.sapharm.2012.02.001v [Epub ahead of print].

Wassenaar, H. J., Chen, W., Cheng, J., \& Sudjianto, A. (2005). Enhancing discrete choice demand modeling for decision-based design. Journal of Mechanical Design, 127(4), 514524. 
Wensing, M., Jung, H. P., Mainz, J., Olesen, F., \& Grol, R. (1998). A systematic review of the literature on patient priorities for general practice care. Part 1: Description of the research domain. Social Science \& Medicine, 47(10), 1573-1588.

Whitty, J. A., Stewart, S., Carrington, M. J., Calderone, A., Marwick, T., Horowitz, J. D., Krum, H., Davidson, P., Macdonald, P., Reid, C., \& Scuffham, P. A. (2013). Patient preferences and willingness-to-pay for a home or clinic based program of chronic heart failure management: findings from the which? Trial. PLoS One, 8(3), e58347. Doi:10.1371/journal.pone.0058347.

Whynes, D. K., Frew, E. J., \& Wolstenholme, J. L. (2005). Willingness-to-pay and demand curves: a comparison of results obtained using different elicitation formats. International Journal of Health Care Finance \& Economics, 5(4), 369-386.

Whynes, D. K., \& Sach, T. H. (2007). WTP and WTA: Do people think differently? Social Science \& Medicine, 65(5), 946-957.

Winkelmann, R. (2004). Co-payments for prescription drugs and the demand for doctor visits-evidence from a natural experiment. Health Economics, 13(11), 1081-1089.

Wiser, R. H. (2007). Using Contingent Valuation to Explore Willingness to Pay for Renewable Energy: A Comparison of Collective and Voluntary Payment Vehicles. Ecological Economics, 62(3-4), 419-432.

the World Bank. (2012). World development indicators: Romania. Retrieved from http://data.worldbank.org/country/romania

the World Bank. (2013). GNI per capita, PPP (current international \$), 2013. Retrieved from http://data.worldbank.org/indicator/NY.GNP.PCAP.PP.CD

World Health Organization (2013). Global Health Expenditure Database: NHA indicators. Retrieved from http://apps.who.int/nha/database/DataExplorerRegime.aspx, Accessed: 17.05.2013.

World Health Organization. (2012). Table of key indicators and sources by country: Ukraine. Retrieved from http://apps.who.int/nha/database/, Accessed: 17.05.2013.

World Health Organization. (2008). The World Health Report 2008: Primary Health Care. Now More Than Ever. Geneva: World Health Organization. 


\section{References}

Wranik, D. (2012). Healthcare policy tools as determinants of health-system efficiency: evidence from the OECD. Health EconomicsPolicy Law, 7(2), 197-226.

Yoo, S.-H., Kim, T.-Y., \& Lee, J.-K. (2001). Modeling zero response data from willingness to pay surveys: A semi-parametric estimation. Economics Letters, 71(2), 191-196.

Zielinski, A., Håkansson, A., Jurgutis, A., Ovhed, I., \& Halling, A. (2008). Differences in referral rates to specialised health care from four primary health care models in Klaipeda, Lithuania. BMC Family Practice, 9(1), 63. Doi: 10.1186/1471-2296-9-63.

Zweifel, P., \& Manning, W. G. (2000). Moral hazard and consumer incentives in health care. In A. J. Culyer \& J. P. Newhouse (Eds.), Handbook of Health Economics (Vol. 1A, pp. 409-459). Amsterdam: North-Holland.

Zweifel, P., Telser, H., \& Vaterlaus, S. (2006). Consumer resistance against regulation: the case of health care. Journal of Regulatory Economics, 29(3), 319-332. 
Appendices 


\section{Appendix A}

\section{Brief methodological report on data collection: Small scale (pilot) survey in Ukraine in 2009}

The survey was subcontracted to Kiev International Institute of Sociology (KIIS). The questionnaire was developed by the research team and adjusted according to the recommendations of KIIS. Prior to the survey, a pre-test on a convenience sample of 55 respondents was conducted. The questions were followed by a feedback from the respondents who described the problems with understanding or answering the questions. Based on the results of this pre-test, the questionnaire, stated preference tasks in particular, was revised and adjusted.

The survey was carried out in December 2009 -January 2010. The fieldwork took place on December 11-28, 2009. An informed consent was obtained from all individuals included in the study. The study instruments and methodology were reviewed by the Institutional Review Board at the National University of Kyiv-Mohyla Academy and a waiver from a full ethical review was obtained.

To get a representative all-Ukrainian sample of 300 interviews for the limited budget of the pilot survey, KIIS proposed to employ a stratified, multi-stage area probability sample of a big nationwide study. This allowed reducing transportation costs. However, information about the non-response rate and, consequently, non-respondents' characteristics was not available.

Thus, the pilot survey interviews were added to the interview chain at the secondary sampling units. The sampling methodology was developed by the sub-contractor. The sample of households from which sample persons were selected, was based on randomly sampled voting precincts within proportionally sampled settlements (e.g., cities towns, villages).

Stage 1: Selection of primary sampling units (settlements)

Ukraine is divided into 24 regions (oblasts), Kyiv and the Crimea. Each oblast is divided into counties (rayons), and one city in each region is designated as the county center. In official statistics, the rural population consists only of villages; the urban population is defined as comprising all cities and urban type villages. The sample was stratified according to whether population points were considered urban or rural. At the first stage of the sample development, 110 primary sampling units were selected by probability proportional to size 
principle. This consisted of 60 cities and towns, 13 urban type villages (PGT) and 36 villages. Nuclear infected territories around the Chernobyl and individuals permanently institutionalized in medical facilities, military quarters, and prisons were not covered in the sampling.

Stage 2: Selection of secondary sampling units (voting precincts)

Voting precincts were used as secondary sample units, because official statistics about them is accessible. They cover all territory of the country, are located more or less evenly, and have moderate size. Within each primary sampling unit, several voting precincts were randomly chosen taking into account the number of residential routes, which is proportional to the number of apartments. The number of selected voting precincts depended on the size of the ultimate clusters in the large nationwide survey. The sample of this pilot survey includes 207 secondary sampling units. 1-2 respondents were interviewed in each secondary sampling unit.

Stage 3: Household and respondent selection

A randomized-number rule was used to select the street, household and apartment that the interviewer was to visit first. Before selecting 'potential respondents', an interviewer enumerates and lists adult (i.e., 18 and older) household members living in a consecutive run of apartments (i.e., from lower to higher apartment numbers) until he or she has a list of occupants sorted by (1) apartment, (2) gender, and (3) age. After generating a list of occupants, the interviewer seeks to recruit every third occupant for the study (i.e., the $3^{\text {rd }}$ occupant is to be interviewed if the assignment for the secondary sampling unit is 1 interview, and the $3^{\text {rd }}$ and the $6^{\text {th }}$ are to be interviewed if the assignment includes 2 interviews).

Overall 303 effective interviews were conducted during the fieldwork stage. 127 interviewers took part in the survey. $10 \%$ of interviews were controlled. That included the control analysis of interviewers' diaries and control phone calls to check the fact and quality of the interviews in the selected households. As a consequence of the control, none of the questionnaires was removed from the final data file. The sample developed for the survey is representative for adult population of Ukraine - the resident population aged 18 and older; it is random at each step of selection. The summary statistics of the sample is presented in Table A.1. 
Table A.1 Socio-demographic characteristics of the sample (303 respondents)

\begin{tabular}{|c|c|c|c|c|}
\hline Characteristic & $\begin{array}{c}\text { Observation } \\
\text { s }\end{array}$ & $\begin{array}{l}\text { Percent } \\
\text { of total }\end{array}$ & $\begin{array}{l}\text { Percent of } \\
\text { non-missing }\end{array}$ & Mean(S.D.) \\
\hline \multicolumn{5}{|l|}{ Age } \\
\hline aged $18-34$ & 72 & $23.76 \%$ & $23.76 \%$ & $47.5 \quad(16.2)$ \\
\hline aged 35-54 & 127 & $41.91 \%$ & $41.91 \%$ & valid $n=303$ \\
\hline aged 55+ & 104 & $34.32 \%$ & $34.32 \%$ & \\
\hline \multicolumn{5}{|l|}{ Sex } \\
\hline Male & 98 & $32.34 \%$ & $32.34 \%$ & \\
\hline female & 205 & $67.66 \%$ & $67.66 \%$ & \\
\hline \multicolumn{5}{|l|}{ Place of residence } \\
\hline village & 122 & $40.26 \%$ & $40.26 \%$ & \\
\hline town (20-500) & 116 & $38.28 \%$ & $38.28 \%$ & \\
\hline big city $(500+)$ or capital & 65 & $21.45 \%$ & $21.45 \%$ & \\
\hline \multicolumn{5}{|l|}{ Education level } \\
\hline lower than secondary & 61 & $20.13 \%$ & $20.20 \%$ & \\
\hline secondary & 174 & $57.43 \%$ & $57.62 \%$ & \\
\hline higher or degree & 67 & $22.11 \%$ & $22.19 \%$ & \\
\hline (missing) & 1 & $0.33 \%$ & & \\
\hline \multicolumn{5}{|l|}{ Health status } \\
\hline absolutely sick to bad & 49 & $16.17 \%$ & $16.17 \%$ & \\
\hline fair & 139 & $45.87 \%$ & $45.87 \%$ & \\
\hline good to perfect & 115 & $37.95 \%$ & $37.95 \%$ & \\
\hline \multicolumn{5}{|l|}{ Voluntary insurance policy } \\
\hline no & 287 & $94.72 \%$ & $95.35 \%$ & \\
\hline yes & 14 & $4.62 \%$ & $4.65 \%$ & \\
\hline (missing) & 2 & $0.66 \%$ & & \\
\hline \multicolumn{5}{|l|}{ Size of the household } \\
\hline 1 member & 41 & $13.53 \%$ & $13.53 \%$ & $3.03 \quad(1.56)$ \\
\hline 2 or more members & 262 & $86.47 \%$ & $86.47 \%$ & valid $n=303$ \\
\hline \multicolumn{5}{|c|}{ Number of children in the household } \\
\hline no children & 181 & $59.74 \%$ & $60.33 \%$ & $0.55 \quad(0.84)$ \\
\hline 1 or more children & 119 & $39.27 \%$ & $39.67 \%$ & valid $n=300$ \\
\hline (missing) & 3 & $0.99 \%$ & & \\
\hline \multicolumn{5}{|c|}{ Share of household members who do not work or earn } \\
\hline $\begin{array}{l}\text { less or one half of family not } \\
\text { working }\end{array}$ & 166 & $54.79 \%$ & $54.97 \%$ & $0.53 \quad(0.35)$ \\
\hline more than half of family not & 136 & $44.88 \%$ & $45.03 \%$ & valid $\mathrm{n}=302$ \\
\hline (missing) & 1 & $0.33 \%$ & & \\
\hline \multicolumn{5}{|l|}{ Income (descriptive) } \\
\hline not sufficient & 105 & $34.65 \%$ & $35.71 \%$ & \\
\hline meets the need & 126 & $41.58 \%$ & $42.86 \%$ & \\
\hline allows saving & 63 & $20.79 \%$ & $21.43 \%$ & \\
\hline (missing) & 9 & $2.97 \%$ & & \\
\hline Total & 303 & $100 \%$ & $100 \%$ & \\
\hline
\end{tabular}


Table A.1 (continued) Socio-demographic characteristics of the sample (303 respondents)

\begin{tabular}{lrrrr}
\hline \multicolumn{1}{c}{ Characteristic } & $\begin{array}{c}\text { Observation } \\
\text { s }\end{array}$ & $\begin{array}{c}\text { Percentage } \\
\text { of total }\end{array}$ & $\begin{array}{c}\text { Percentage } \\
\text { of } \\
\text { non-missing }\end{array}$ & Mean(S.D.) \\
\hline Income (level, UAH) & 58 & $19.14 \%$ & $21.72 \%$ & $2346.8 \quad(1781.1)$ \\
$\quad$ 1000 UAH or less & 104 & $34.32 \%$ & $38.95 \%$ & valid n = 267 \\
from 1001 to 2000 UAH & 66 & $21.78 \%$ & $24.72 \%$ & \\
from 2001 to 4000 UAH & 39 & $12.87 \%$ & $14.61 \%$ & \\
$\quad$ 4001 UAH and more & 36 & $11.88 \%$ & & \\
$\quad$ (missing) & 55 & $18.15 \%$ & $18.97 \%$ & \\
\hline Experience in visiting and paying to a physician & 101 & $33.33 \%$ & $34.83 \%$ & \\
$\quad$ did not visit & 134 & $44.22 \%$ & $46.21 \%$ & \\
$\quad$ visited did not pay & 13 & $4.29 \%$ & & \\
$\quad$ visited and paid & 303 & $100 \%$ & $100 \%$ & \\
$\quad$ (missing) & & & & \\
\hline Total & & & & \\
\hline
\end{tabular}




\section{Appendix B}

\section{Brief methodological report on data collection: Survey from six CEE countries in $2010^{11}$}

The data collection took a form of household survey carried out in July 2010 in six CEE countries - Bulgaria, Lithuania, Hungary, Poland, Romania and Ukraine. The data collection was sub-contracted to Gallup International. The subcontractor used the survey questionnaire developed by the research team. The questionnaire and its translations were adjusted based on recommendations by the sub-contractor. The sub-contractor was responsible for the preparation of the data collection, the data-collection and the creation of the database.

The objective of the survey was to provide quantitative data on past payments for health care services, data on preferences and willingness to pay for health care improvements. The objective also was to provide data comparable across the countries. For this purpose, the questionnaire for all countries was identical, and the data collection process took place simultaneously in all six countries in a compact period of time - 20 calendar days. In all countries, the surveys were conducted based on face-to-face individual interviews. The respondents were identified using the same sampling methodology for all countries. The aim was to have 1000 effective interviews per country that present samples representativeness of the countries.

The sampling methodology was developed by the sub-contractor. It was based on a multistaged random probability method:

Stage 1: Distribution of sampling points.

The sampling points in each country were distributed proportionally to regional, urban/rural and ethnic characteristics of the population. Within each region, the cities and towns belonging to the same group were put in an alphabetical order. The cities and towns included in the survey, were selected at random from that list. The number of sampling points in the

\footnotetext{
${ }^{11}$ The survey represented the first wave of data collection held within the framework of the International Project ASSPRO CEE 2007 (Assessment of patient payment policies and projection of their efficiency, equity and quality effects: The case of Central and Eastern Europe). The Project was financed by the European Commission under FP7 Theme 8 Socio-economic Sciences and Humanities, Grant Agreement no. 217431. For details see: www.assprocee2007.com
} 
rural areas was calculated based on the ratio urban/rural population in a country. In total, there were ca. 150 sampling points per country.

Stage 2: Selection of addresses/ households.

The objective was to identify, 8-10 respondents per sampling point. To select addresses/households of potential respondents, the random route method was used. For each sampling point, a starting point and direction were determined. The household selected for the survey was every forth address on the left-hand side of the street in urban areas, turning left at intersections and, after reaching a dead end, going back to the last crossing and further proceeding at random. In a block-of-flats of up to four floors, every fifth apartment household was selected, counting from the first apartment on the left of the ground floor. In cases of unsuitable household, the interviewers approached the apartment next-door and continued doing this until reaching a suitable household. At that point, the interviews resume the standard step of every fifth apartment. In a block-of-flats of 5 floors and more, the selection is every tenth apartment. In rural areas, every fourth inhabitable house on both sides of the interviewer's route was selected. In compounds of several houses behind a common fence, the interviewer had to select the fourth one from the left (counting from the gate), or if there were less than four houses behind a common fence, then the interviewer went out of the common yard, counting the houses as if they were along the street.

Stage 3: Selection of the respondent within the household selected.

The selection of the respondent within the selected household was done by using the "last birthday” principle. In this procedure, the interviewer asked to speak to the adult member of the household who had the last birthday. The last-birthday method is based on the assumption that the assignment of birthdates is a random process and also every household member has an equal chance of being selected. Only one individual per household was interviewed.

Stage 4: Replacement of the respondent/household.

If the respondent determined on stage 3 refused or was unavailable to take part in an interview after two call backs recorded in the fieldwork report, a replacing respondent was identified following stage 2-3. The sampling procedure described above, is known as an efficient method for selecting a sample representative for the population for a particular country. It is proven by practice that the sample produced by this method does not differ significantly from the official statistical data on age, gender and other demographic parameters. The subcontractor organised and managed the interviewers' training to clarify the fieldwork standards 
and the specificities of the questionnaire. A high number of interviewers were involved in the survey to avoid the interviewer bias that might occur when one interviewer carries out many interviews.

The final number of effective interviews in the dataset, number of interviewers per country, and median duration of interview are presented in Table B.1. The duration of the interviews was as expected - on average 30 min per interview. Number of effective interviews is no less than 1000 in each country.About $10 \%$ of all interviews per country were verified (recontacted) either by telephone or in person by the sub-contractor. The verifications of the interviews confirmed that that the interviews were carried out in reality. The sub-contractor entered the data collected according to the preliminary data entry mask consulted and agreed with the research team.

Table B.1 Short summary of the results of data collection

\begin{tabular}{|l|c|c|c|c|c|c|}
\hline & Bulgaria & Hungary & Lithuania & Poland & Romania & Ukraine \\
\hline $\begin{array}{l}\text { Number of effective } \\
\text { interviews (respondents) }\end{array}$ & 1003 & 1037 & 1012 & 1000 & 1000 & 1000 \\
\hline $\begin{array}{l}\text { Total number } \\
\text { of interviewers }\end{array}$ & 108 & 130 & 84 & 70 & 100 & 111 \\
\hline $\begin{array}{l}\text { Median duration of } \\
\text { interviews (minutes) }\end{array}$ & 35.00 & 35.00 & 33.00 & 27.00 & 30.00 & 32.00 \\
\hline
\end{tabular}

The sub-contractor also performed the standard data cleanup and logical checks procedures. The research team checked the representativeness of the sample and the overall quality of the dataset. The sample characteristics related to age, gender place of residence and household income are overall comparable to the countries' national statistics. The descriptive statistics of the samples are presented in Table B.2.

Table B.2 Descriptive characteristics of the study samples

\begin{tabular}{llcccccc}
\hline & Bulgaria & Hungary & Lithuania & Poland & Romania & Ukraine \\
\hline \multicolumn{2}{l}{ Total number of the respondents } & 1003 & 1037 & 1012 & 1000 & 1000 & 1000 \\
\hline \multicolumn{2}{l}{ Categorical characteristics } & & & & & & \\
\hline Sex & male & $46.86 \%$ & $46.38 \%$ & $43.18 \%$ & $47.00 \%$ & $41.70 \%$ & $41.50 \%$ \\
& female & $53.14 \%$ & $53.62 \%$ & $56.82 \%$ & $53.00 \%$ & $58.30 \%$ & $58.50 \%$ \\
\cline { 2 - 7 } Place of & village & $29.81 \%$ & $29.22 \%$ & $33.60 \%$ & $37.00 \%$ & $43.80 \%$ & $31.50 \%$ \\
residence & town & $44.37 \%$ & $50.92 \%$ & $39.13 \%$ & $40.00 \%$ & $33.70 \%$ & $32.80 \%$ \\
& city & $25.82 \%$ & $19.86 \%$ & $27.27 \%$ & $23.00 \%$ & $22.50 \%$ & $35.70 \%$ \\
\cline { 2 - 7 } & & & & & & &
\end{tabular}


Table B.2 (continued) Descriptive characteristics of the study samples

\begin{tabular}{|c|c|c|c|c|c|c|c|c|}
\hline & & & Bulgaria & Hungary & Lithuania & Poland & Romania & Ukraine \\
\hline \multicolumn{9}{|c|}{ Categorical characteristics } \\
\hline $\begin{array}{l}\text { Education } \\
\text { level }\end{array}$ & \multicolumn{2}{|c|}{$\begin{array}{l}\leq \text { lower/secondary } \\
\text { upper secondary } \\
\geq \text { post-secondary }\end{array}$} & $\begin{array}{l}22.93 \% \\
53.84 \% \\
23.23 \%\end{array}$ & $\begin{array}{l}51.01 \% \\
32.50 \% \\
16.49 \%\end{array}$ & $\begin{array}{l}12.65 \% \\
42.89 \% \\
44.47 \%\end{array}$ & $\begin{array}{l}25.05 \% \\
61.22 \% \\
13.73 \%\end{array}$ & $\begin{array}{l}23.10 \% \\
50.70 \% \\
26.20 \%\end{array}$ & $\begin{array}{r}.30 \% \\
62.40 \% \\
30.30 \%\end{array}$ \\
\hline $\begin{array}{l}\text { Self-reported } \\
\text { health status }\end{array}$ & \multicolumn{2}{|c|}{$\begin{array}{l}\text { very bad or bad } \\
\text { Fair } \\
\text { Good } \\
\text { very good/perfect }\end{array}$} & $\begin{array}{l}13.81 \% \\
27.33 \% \\
33.13 \% \\
25.73 \%\end{array}$ & $\begin{array}{l}11.86 \% \\
27.39 \% \\
34.23 \% \\
26.52 \%\end{array}$ & $\begin{array}{r}9.29 \% \\
37.55 \% \\
33.20 \% \\
19.96 \%\end{array}$ & $\begin{array}{l}12.20 \% \\
25.90 \% \\
32.50 \% \\
29.40 \%\end{array}$ & $\begin{array}{l}15.90 \% \\
29.80 \% \\
38.40 \% \\
15.90 \%\end{array}$ & $\begin{array}{r}24.00 \% \\
49.20 \% \\
19.00 \% \\
7.80 \%\end{array}$ \\
\hline $\begin{array}{l}\text { Chronic } \\
\text { disease }\end{array}$ & \multicolumn{2}{|c|}{$\begin{array}{l}\text { any } \\
\text { diabetes } \\
\text { cardio-vascular } \\
\text { kidney,liver,lung } \\
\text { other problems }\end{array}$} & $\begin{array}{r}47.36 \% \\
8.20 \% \\
33.60 \% \\
10.10 \% \\
23.94 \%\end{array}$ & $\begin{array}{r}45.13 \% \\
10.51 \% \\
31.63 \% \\
5.59 \% \\
20.93 \%\end{array}$ & $\begin{array}{r}47.33 \% \\
4.94 \% \\
27.96 \% \\
10.67 \% \\
29.15 \%\end{array}$ & $\begin{array}{r}35.50 \% \\
6.50 \% \\
19.50 \% \\
4.50 \% \\
21.50 \%\end{array}$ & $\begin{array}{r}42.60 \% \\
7.00 \% \\
28.50 \% \\
12.70 \% \\
21.40 \%\end{array}$ & $\begin{array}{r}46.00 \% \\
3.50 \% \\
27.10 \% \\
14.50 \% \\
28.50 \%\end{array}$ \\
\hline $\begin{array}{l}\text { Perceived } \\
\text { level of } \\
\text { income }\end{array}$ & \multicolumn{2}{|c|}{$\begin{array}{l}\text { not sufficient } \\
\text { meets expenses } \\
\text { allows saving }\end{array}$} & $\begin{array}{r}36.09 \% \\
55.02 \% \\
8.89 \%\end{array}$ & $\begin{array}{l}24.61 \% \\
48.25 \% \\
27.14 \%\end{array}$ & $\begin{array}{l}23.06 \% \\
47.32 \% \\
29.62 \%\end{array}$ & $\begin{array}{l}15.50 \% \\
48.97 \% \\
35.52 \%\end{array}$ & $\begin{array}{l}38.24 \% \\
43.09 \% \\
18.67 \%\end{array}$ & $\begin{array}{l}29.11 \% \\
55.67 \% \\
15.22 \%\end{array}$ \\
\hline $\begin{array}{l}\text { Willingness to } \\
\text { pay }\end{array}$ & \multicolumn{2}{|c|}{$\begin{array}{l}\text { Willing } \\
\text { (s.e.) }\end{array}$} & $\begin{array}{r}75.96 \% \\
1.36 \%\end{array}$ & $\begin{array}{r}66.54 \% \\
1.47 \%\end{array}$ & $\begin{array}{r}80.90 \% \\
1.24 \%\end{array}$ & $\begin{array}{r}72.96 \% \\
1.41 \%\end{array}$ & $\begin{array}{r}81.43 \% \\
1.24 \%\end{array}$ & $\begin{array}{r}73.47 \% \\
1.40 \%\end{array}$ \\
\hline & \multicolumn{2}{|c|}{$\begin{array}{l}\text { Not willing } \\
\text { (s.e.) }\end{array}$} & $\begin{array}{r}24.04 \% \\
1.36 \%\end{array}$ & $\begin{array}{r}33.46 \% \\
1.47 \%\end{array}$ & $\begin{array}{r}19.10 \% \\
1.24 \%\end{array}$ & $\begin{array}{r}27.04 \% \\
1.41 \%\end{array}$ & $\begin{array}{r}18.57 \% \\
1.24 \%\end{array}$ & $\begin{array}{r}26.53 \% \\
1.40 \%\end{array}$ \\
\hline \multicolumn{9}{|c|}{ Continuous characteristics } \\
\hline \multicolumn{2}{|l|}{ Age, years } & $\begin{array}{r}\text { mean } \\
(\text { s.e. })\end{array}$ & $\begin{array}{c}50.49 \\
0.54\end{array}$ & $\begin{array}{c}46.34 \\
0.55\end{array}$ & $\begin{array}{c}46.38 \\
0.53\end{array}$ & $\begin{array}{c}44.12 \\
0.52\end{array}$ & $\begin{array}{c}48.49 \\
0.54\end{array}$ & $\begin{array}{c}48.56 \\
0.56\end{array}$ \\
\hline \multicolumn{2}{|c|}{$\begin{array}{l}\text { Monthly household } \\
\text { income, } €\end{array}$} & $\begin{array}{r}\text { mean } \\
\text { (s.e.) }\end{array}$ & $\begin{array}{c}358.01 \\
8.77\end{array}$ & $\begin{array}{c}587.10 \\
10.29\end{array}$ & $\begin{array}{c}531.40 \\
13.93\end{array}$ & $\begin{array}{c}733.16 \\
14.31\end{array}$ & $\begin{array}{c}357.34 \\
10.61\end{array}$ & $\begin{array}{c}252.66 \\
5.32\end{array}$ \\
\hline \multicolumn{2}{|c|}{$\begin{array}{l}\text { Size of the household, } \\
\text { members }\end{array}$} & $\begin{array}{r}\text { mean } \\
\text { (s.e.) }\end{array}$ & $\begin{array}{l}2.90 \\
0.04\end{array}$ & $\begin{array}{l}2.66 \\
0.04\end{array}$ & $\begin{array}{l}2.61 \\
0.04\end{array}$ & $\begin{array}{l}3.10 \\
0.05\end{array}$ & $\begin{array}{l}2.63 \\
0.04\end{array}$ & $\begin{array}{l}2.83 \\
0.04\end{array}$ \\
\hline \multicolumn{2}{|c|}{$\begin{array}{l}\text { Monthly income } \\
\text { per person, } €\end{array}$} & $\begin{array}{r}\text { mean } \\
\text { (s.e.) }\end{array}$ & $\begin{array}{c}135.87 \\
3.14\end{array}$ & $\begin{array}{c}252.15 \\
4.33\end{array}$ & $\begin{array}{c}225.73 \\
5.54\end{array}$ & $\begin{array}{c}273.81 \\
5.45\end{array}$ & $\begin{array}{c}157.82 \\
5.70\end{array}$ & $\begin{array}{c}101.02 \\
2.43\end{array}$ \\
\hline \multicolumn{2}{|c|}{ Number of children } & $\begin{array}{r}\text { mean } \\
(\text { s.e. })\end{array}$ & $\begin{array}{l}0.44 \\
0.02\end{array}$ & $\begin{array}{l}0.47 \\
0.03\end{array}$ & $\begin{array}{l}0.56 \\
0.03\end{array}$ & $\begin{array}{l}0.61 \\
0.03\end{array}$ & $\begin{array}{l}0.50 \\
0.03\end{array}$ & $\begin{array}{l}0.56 \\
0.03\end{array}$ \\
\hline \multicolumn{2}{|c|}{$\begin{array}{l}\text { Number of members } \\
\text { with chronic disease }\end{array}$} & $\begin{array}{r}\text { mean } \\
\text { (s.e.) }\end{array}$ & $\begin{array}{l}0.80 \\
0.81\end{array}$ & $\begin{array}{l}0.61 \\
0.76\end{array}$ & $\begin{array}{l}0.63 \\
0.76\end{array}$ & $\begin{array}{l}0.59 \\
0.77\end{array}$ & $\begin{array}{l}0.57 \\
0.73\end{array}$ & $\begin{array}{l}0.77 \\
0.81\end{array}$ \\
\hline \multicolumn{9}{|c|}{ Payment experience in the past year for outpatient physian services } \\
\hline \multicolumn{2}{|c|}{ Number of visits } & $\begin{array}{r}\text { mean } \\
\text { (s.e.) }\end{array}$ & $\begin{array}{l}4.48 \\
0.22\end{array}$ & $\begin{array}{l}5.26 \\
0.19\end{array}$ & $\begin{array}{l}3.67 \\
0.16\end{array}$ & $\begin{array}{l}3.78 \\
0.15\end{array}$ & $\begin{array}{l}3.24 \\
0.14\end{array}$ & $\begin{array}{l}1.98 \\
0.12\end{array}$ \\
\hline \multicolumn{2}{|c|}{$\begin{array}{l}\text { All types of payments, } \\
€\end{array}$} & $\begin{array}{r}\text { mean } \\
(\text { s.e. })\end{array}$ & $\begin{array}{c}16.81 \\
2.46\end{array}$ & $\begin{array}{c}16.85 \\
1.90\end{array}$ & $\begin{array}{c}26.98 \\
3.09\end{array}$ & $\begin{array}{c}14.35 \\
1.56\end{array}$ & $\begin{array}{c}48.92 \\
8.76\end{array}$ & $\begin{array}{c}19.91 \\
2.43\end{array}$ \\
\hline \multicolumn{2}{|c|}{ Informal payments, € } & $\begin{array}{r}\text { mean } \\
(\text { s.e. })\end{array}$ & $\begin{array}{l}3.40 \\
1.37\end{array}$ & $\begin{array}{l}9.99 \\
1.20\end{array}$ & $\begin{array}{c}11.77 \\
2.14\end{array}$ & $\begin{array}{l}3.48 \\
0.76\end{array}$ & $\begin{array}{c}14.68 \\
2.94\end{array}$ & $\begin{array}{l}6.81 \\
1.01\end{array}$ \\
\hline \multicolumn{2}{|c|}{ Official payments, $€$} & $\begin{array}{r}\text { mean } \\
\text { (s.e.) }\end{array}$ & $\begin{array}{c}13.18 \\
2.00\end{array}$ & $\begin{array}{l}6.96 \\
1.05\end{array}$ & $\begin{array}{c}15.19 \\
1.88\end{array}$ & $\begin{array}{c}10.79 \\
1.20\end{array}$ & $\begin{array}{c}33.90 \\
6.62\end{array}$ & $\begin{array}{c}13.03 \\
1.85\end{array}$ \\
\hline \multicolumn{9}{|c|}{ Willingness and ability to pay for the services of medical specialists } \\
\hline \multicolumn{2}{|c|}{ All zeros included, € } & $\begin{array}{r}\text { mean } \\
\text { (s.e.) } \\
\text { median }\end{array}$ & $\begin{array}{l}6.63 \\
0.23 \\
7.50\end{array}$ & $\begin{array}{l}9.05 \\
0.58 \\
5.26\end{array}$ & $\begin{array}{l}9.63 \\
0.43 \\
7.43\end{array}$ & $\begin{array}{c}10.97 \\
0.45 \\
9.76\end{array}$ & $\begin{array}{l}8.94 \\
0.42 \\
6.98\end{array}$ & $\begin{array}{l}4.92 \\
0.23 \\
4.12\end{array}$ \\
\hline \multicolumn{2}{|c|}{ Only positive, $€$} & $\begin{array}{r}\text { mean } \\
\text { (s.e.) } \\
\text { median }\end{array}$ & $\begin{array}{l}8.79 \\
0.25 \\
7.50\end{array}$ & $\begin{array}{c}13.91 \\
0.83 \\
10.00\end{array}$ & $\begin{array}{c}11.95 \\
0.50 \\
8.57\end{array}$ & $\begin{array}{c}15.11 \\
0.54 \\
12.20\end{array}$ & $\begin{array}{c}11.16 \\
0.50 \\
7.44\end{array}$ & $\begin{array}{l}6.77 \\
0.29 \\
5.15\end{array}$ \\
\hline
\end{tabular}




\section{Appendix C}

\section{Examples of the valuation tasks used in the small scale survey in Ukraine in 2009}

\section{An example of a discrete choice task}

Question: Imagine you have some major or severe symptoms.

[Description for the interviewer: Major or severe symptoms are those that are unfamiliar or badly familiar to you, you are anxious if they pass or aggravate, they may cause discomfort and prevent from usual activities, you may have pain up to very severe. These symptoms make you think of referral to a physician].

Given this and taking into account your real life situation, which of the physicians from each pair (A or B) would you choose if you met the following choices in the real market? Please perform all 16 choice tasks.

Characteristics that differ across two physicians are bolded.

\section{Choice \#}

\begin{tabular}{|c|c|}
\hline $\begin{array}{l}\text { Physician A } \\
\text { - } \quad \text { General practitioner [district/family } \\
\text { - } \text { doctor/internist] } \\
\text { - } \quad \text { Outdated medical equipment } \\
\text { - } \quad \text { Polite treatment of medical staff } \\
\text { - } 15 \text { min travelling to the office } \\
\text { - } \quad \mathbf{4 5} \text { min waiting in front of the office } \\
\text { - } 20 \text { UAH out of the patient's pocket } \\
\text { - } \quad \text { Informal payment }\end{array}$ & $\begin{array}{l}\text { Physician B } \\
\text { - } \quad \text { General practitioner [district/family } \\
\text { - } \text { doctor/internist] } \\
\text { - } \quad \text { Out-dated medical equipment } \\
\text { - } \quad \text { Arrogant treatment of medical staff } \\
\text { - } 15 \text { min travelling to the office } \\
\text { - } 10 \text { min waiting in front of the office } \\
\text { - } 20 \text { UAH out of the patient's pocket } \\
\text { - } \quad \text { Informal payment }\end{array}$ \\
\hline \multicolumn{2}{|c|}{$\begin{array}{l}\text { If you had to choose what physician would you choose? } \\
\square \mathrm{A} \square \mathrm{B}\end{array}$} \\
\hline
\end{tabular}

\section{An example of a contingent valuation task}

Question If the Government would invest into health care so that characteristics of the abovementioned physician would improve to the following:

- General practitioner [district doctor / internist, family doctor]

- Modern medical equipment

- Renovated physician's office

- Polite treatment of medical staff

- 15 min travelling to the office

- 10 min waiting in front of the office

would you be willing to pay official fee for each visit?

If no: What is the reason for your unwillingness to pay?

$1=$ Not able to pay

$2=$ Object to pay for this medical service

$3=$ Not able to pay and object to pay for this medical service

If yes: What is the maximum amount of money that you are willing to pay?

$1=$ Less than $20 \mathrm{UAH}$

$2=$ Between 20 and $60 \mathrm{UAH}$

$3=$ Between 60 and $100 \mathrm{UAH}$

$4=$ More than $100 \mathrm{UAH}$

How much exactly? 


\section{Appendix D}

\section{An example of a CV task used in the full scale survey in six CEE countries in 2010.}

Next questions concern your WILLINGNESS and ABILITY to pay for medical services provided by the state or included in the social health insurance package. Imagine that you could obtain these services with GOOD QUALITY and QUICK ACCESS if you pay an OFFICIAL FEE to the health care facility (e.g. polyclinic, clinic or hospital).

\section{SHOW CARD}

This card presents the meaning of good quality and quick access.

GOOD QUALITY would mean:

- Modern medical equipment

- Renovated health care facility

- Polite staff with good reputation and skills

QUICK ACCESS would mean:

- Max 30 min travelling to the health care facility

- Max 10 min waiting in front of the physician office

- Max 1 month waiting for a planned surgery

At the bottom of the card, you can also see possible fees intervals regarding physician's and hospital services. Higher fee would mean better quality and quicker access. Fees are fixed prices and are not necessarily dependent on the service costs, since a part of the costs are covered by public funds.

Q.1 In case you experience a MAJOR HEALTH PROBLEM (unfamiliar symptoms that make you concerned), will you be willing to pay an official fee for a consultation and examination by a MEDICAL SPECIALIST in order to obtain services with good quality and quick access as described in the card?

$$
\begin{array}{ll}
\text { YES } & \text {-> go to Q.2 } \\
\text { NO } & \text {-> skip Q.2 }
\end{array}
$$

Q.2 Considering the fee intervals regarding physician's services shown on the card, how much exactly are you WILLING and ABLE to pay for such visit in order to obtain services with good quality and quick access?

If the respondents states an interval, ask for an exact amount. If the respondent is not able to indicate an exact amount, fill in the midpoint of the interval.

\section{Midpoints:}

Less than 5.- Euro

From 5.- to 10.- Euro

(midpoint 2.50 Euro)

More than 10.- Euro

(midpoint 7.50 Euro)

(midpoint 12.50 Euro) 



\section{Summary}

Willingness to pay derived using stated preference techniques (i.e. valuation methods, assessing willingness to pay for certain benefits in hypothetical purchase or choice scenarios) is increasingly used for the valuation of health care benefits. The use of stated preference techniques is conditioned by their ability to provide monetary values for previously unknown or non-traded benefits for which no proxy markets are known. Another advantage is that stated preference techniques capture passive use values, i.e. utility obtained from the mere availability of a benefit without usage, which is especially relevant in health care.

Nonetheless, stated preference techniques have gained very limited space in the assessment of patient charges and their design. This might be due to uncertainty whether stated willingness to pay actually reflects the willingness to pay in real world decisions. This uncertainty is known as hypothetical bias. Moreover, the two main stated preference techniques, discrete choice experiments and contingent valuation, do not demonstrate convergence in their valuation measures.

Despite the abovementioned problems, stated preference techniques, if appropriately designed, are recognized as a valid tool for the valuation of environmental benefits. By the same token, in many health care systems, where health benefits have never been traded, stated preference techniques are the only way of assessing patients' willingness to pay. This is the case in many post-socialist Central and Eastern European countries where formal patient charges for health care services are not common.

Patient charges for physician services are advised as an efficient policy tool for the reduction of unnecessary use of services, superseding informal charges (which are widespread in Central and Eastern European countries) and to some extent as an additional source of health care funding. In Central and Eastern Europe, however, in the post-socialistic context, many countries are reluctant to undertake such unpopular measures as the introduction of formal charges for physician visits. This ignorance, however, lacks evidence from the assessment of patient charges and their effects. Most of the evidence is based on revealed preference data (i.e. data or past service utilization) and, hence, comes from Western Europe, USA, and some Asian countries, where patient charges are already effectively implemented. For Central and Eastern European countries such evidence is lacking. 
As outlined in Chapter 1, the overall aim of this dissertation is to apply stated preference techniques in order to study the willingness to pay for physician services in Central and Eastern European countries, and to analyze to what extent willingness to pay estimates can be used in the assessment of patient charges for physician services. The following countries are included in the study: Bulgaria, Hungary, Lithuania, Poland, Romania and Ukraine. These countries present a relevant context for a cross-country comparison given the past similarities in their health care sectors during the communist period, as well as the different pace of health care reforms during the post-communist era.

For the purpose of the dissertation, the following objectives are defined:

1. To investigate aspects of the value added of physician services by determining the marginal willingness to pay for service quality and access characteristics using a discrete choice experiment technique.

2. To study the socio-economic and demographic drivers of the willingness to pay and level of the willingness to pay for physician services using a contingent valuation technique.

3. To compare the willingness to pay estimates from the two main stated preferences techniques, i.e. discrete choice experiment and contingent valuation, in terms of their consistency.

4. To use the stated willingness to pay values to define the potential demand pools at different levels of patient charges for physician services.

5. To assess the possible overestimation of the willingness to pay by the respondents using calibration techniques.

6. To compare willingness to pay estimates with service cost and actual payments.

7. To discuss the implications of the willingness to pay estimates for determining feasible levels of patient charges for physician services and for the overall assessment of patient payment policies.

The above objectives are applied to physician services in the health care systems of the six Central and Eastern European countries mentioned above. In all countries, except for Ukraine, social health insurance systems have been introduced. In Ukraine, a publicly funded system (the so called Semashko system of USSR) still exists. In most of these countries there are 
patient charges for commodities (pharmaceuticals and devices). However, patient charges for basic health care services are not common. Only in Bulgaria, a relatively low fee of around $€ 1$ for a visit to a physician was introduced in 2000 and still functions. In Hungary, a small visit fee of about $€ 1$ existed for about one year in 2007 but was abolished at a national referendum in 2008. In the other four countries, formal patient charges during the time this research was conducted were applied only to the services outside the basic service package. In all countries however, quasi-formal charges set by health care providers in the absence of clear government regulations, as well as informal (under-the-table) payments exist at all levels of health care provision, including physician services.

The first three objectives of the dissertation are dealt with by an application of discrete choice experiments and contingent valuation tasks in a small scale survey in Ukraine. Chapters 2 through 4 are devoted to the analysis of the data collected in this survey. The fourth and the fifth objectives are the main research questions in Chapters 5 and 6 of this dissertation respectively. To tackle these objectives at a cross-country level, we use the willingness to pay estimates from the contingent valuation tasks in the representative surveys held in the six Central and Eastern European countries in July-August 2010. The sample in each country is representative of the adult population of the country and contains around 1000 respondents. Calibration is conducted by applying a stochastic frontier model. Potential demand pools are built by the application of a semi-parametric survival analysis to willingness to pay data. Eventually, the results of the two last chapters allow addressing the two last objectives of this dissertation. More broadly, the relevance and reliability of these results are addressed in the general discussion of the dissertation (Chapter 7).

Chapter 2 provides evidence on the preferences of Ukrainian consumers for health care improvements, which can help to design reforms that reflect societal priorities. In particular, Chapter 2 aims to elicit and to place monetary values on public preferences for outpatient physician services in Ukraine. The method of discrete choice experiment is used on a sample of 303 respondents, representative for the Ukrainian population. The random effect logit model with interactions provides the best fit for the data and is used to calculate the marginal willingness to pay for quality and access improvements. 
At a sample level, there is no clear preference to pay formally rather than informally or vice versa. We also do not find that visiting a general practitioner is preferred over direct access to a medical specialist. However, there are differences between population groups. Qualityrelated attributes of physician services appear to be important to respondents, especially the attitude of medical staff. Thus, the improvement of interpersonal aspects of outpatient care should be given priority when deciding on investments in quality improvements in Ukraine. Other aspects, i.e. social quality and access, are important as well but their improvement brings fewer social gains.

Chapter 3 focuses on the potential and feasibility of official patient charges for public health care services in Ukraine by studying the patterns of fee acceptability, ability and willingness to pay for public health care across population groups. We use contingent valuation data collected from 303 respondents representative of the adult Ukrainian population. Three decision points were separated: objection to pay, inability to pay, and level of positive nonzero willingness to pay. These decisions were studied for relations with quality profiles of the services, and socio-demographic characteristics of the respondents and their households.

The likelihood to object to pay is found to be mostly determined by quality characteristics of the services. Objection to pay is not related to respective behavior in real life. The likelihood of being unable to pay is associated with older age, a larger share of nonearning household members and a lower income. The level of positive willingness to pay is positively related to income (+7\% per $1000 \mathrm{UAH}$ increase in income) and is lower for people who visited a physician but did not pay (-22\%). The rather substantial willingness to pay levels (between $0.9 \%$ and $1.9 \%$ of household income) for one visit to a physician, indicate a potential for official patient charges in Ukraine. The patterns of inability to pay support the need of exemption criteria based on age, income, and other aspects of economic status. The willingness to pay patterns highlight the necessity for payments that are proportional to income.

As explained in Chapter 4, discrete choice experiments and contingent valuation are often applied to value health care benefits. However, whether the two techniques yield converging willingness to pay estimates is not studied well. Thus, Chapter 4 aims to compare at a disaggregated level the willingness to pay estimates for physician services obtained from a 
discrete choice experiment and from a contingent valuation study (the two studies presented in Chapter 2 and 3). We study the consistency between the estimates and whether there are systematic differences between the two types of estimates. The same as for Chapter 2 and 3, the analysis is based on data from a household survey in Ukraine that includes 303 respondents and is taken to be representative of the Ukrainian population. The respondents participated in both the discrete choice experiment (16 choice tasks) and contingent valuation (4 valuation scenarios) in the form of a payment scale followed by open-ended questions about the exact maximum willingness to pay.

We find that the discrete choice experiment produces higher willingness to pay estimates than the contingent valuation does, and the estimates are not consistent across the two techniques. A difference between the willingness to pay estimates from the discrete choice experiment over those derived from the contingent valuation technique is found (i) for respondents who do not discriminate well between the profiles, and (ii) for an increase in the presented attribute level changes. The implications for achieving better convergence between the willingness to pay estimates from the two techniques are discussed.

As outlined in Chapter 5, patient charges for health care services are implemented in developed countries to reduce unnecessary service use. However, evidence on their potential effects in Central and Eastern Europe is lacking. Thus, in Chapter 5, we provide evidence on the potential impact of patient charges on the consumption of specialized physician services in the six Central and Eastern European countries included in this dissertation: Bulgaria, Hungary, Lithuania, Poland, Romania, and Ukraine. We apply a semi-parametric survival analysis to the stated willingness and ability to pay in order to identify potential demand pools, i.e. shares of population willing and able to pay a certain fee in case they need a service, and we also calculate price, income and age semi-elasticities. Data are collected through national surveys held in 2010 among representative samples of about 1000 respondents in each of the six Central and Eastern European countries included in the analysis.

Our results suggest that median willingness and ability to pay in the studied countries ranges from $€ 5.15$ to $€ 12.2$ and the country ranking by willingness and ability to pay follows exactly the ranking by income level. Low service charges, up to $€ 2.5$ in Bulgaria, Hungary, Lithuania and Romania, and up to $€ 5$ in Poland should not cause many people to drop out of 
the demand pool. The lower payment interval should be studied in more detail for Ukraine, however, with a more sensitive scale. Official service charges together with exemption/reduction criteria are argued to be beneficial as an alternative to informal payments.

Chapter 6 is devoted to the proper assessment of skewed willingness to pay data for specialized physician services. The analysis is based on the same data as in Chapter 5, i.e. contingent valuation data from six CEE countries: Bulgaria, Hungary, Lithuania, Poland, Romania, and Ukraine. The willingness to pay levels are modeled with a stochastic frontier regression accounting for positive and negative skewness, assumed to be representing overstatement and understatement of the true level of willingness and ability to pay respectively. The willingness to pay levels are calibrated based on individual predictions of the inefficiency scores. The raw and calibrated willingness to pay levels are compared to actual expenditure by making annual predictions for expenditure if respondents were actually paying amounts equal to their willingness to pay.

Our results suggest that the skewness of willingness to pay across countries is explained by the interaction of three main factors: the perceived impact on actual policy, relevance and desirability of the valued changes, and past use of the services. In countries, where obligatory patient charges are in effect and, consequently, price increases are likely, respondents might conceal their 'true willingness to pay', especially the users. In countries where actual price increases are unlikely to happen, in contrast, the desire for improvements might lead to overstatement of the 'true willingness to pay', especially infrequent or non-users. Despite the adopted estimate, i.e. raw or calibrated, Ukrainians and Romanians actually spend more on current services than their maximal willingness to pay for services with high quality and quick access which indicates that current unregulated charges impose a substantial burden in these countries.

Chapter 7 presents the discussion of the key findings outlined in Chapter 2 to 6 . They are divided into two main parts: those related to the use of the stated preferences for the assessment of patient payments, and to the policy implications for the design of patient payments in the six Central and Eastern European countries. 
As for the use of the stated preference techniques, there is still much to be done in improving their applicability. The consistency of the willingness to pay estimates obtained from the discrete choice experiment and contingent valuation techniques might be improved by increasing the importance of the tasks to the respondents or accounting for the certainty with which the respondents make choices. Additionally, strategies for improving consistency might imply also assessment of the marginal (small) changes in the benefits or application of nonlinear utility functions. In any case, we believe, discrete choice experiment estimates should not be used for obtaining holistic measures of willingness to pay.

We also discuss that stated willingness to pay is jointly influenced by consequences for future policy perceived by the respondents, and by past respondents' experience with payments and use of care. Thus, as discussed in Chapter 6, strategic answering patterns (under- and overstatement) might be seen as the result of a trade-off a respondent makes between the desirability of changes described in the valuation task (i.e. quality and access improvements) and the likelihood of an actual price increase as a consequence of the survey perceived by the respondent. Our results suggest that there is much more heterogeneity in stated willingness to pay than that which could be explained by observed factors, and part of it is related to the perceived likelihood of payment changes as a consequence of the survey.

In this dissertation, we also discuss that different mechanisms underlie the decision to be willing to pay and the level of willingness to pay. Our results support the current practice in the contingent valuation literature of dealing with the stated willingness-to-pay level and willingness to pay as separate decision steps. We find that the question about the motivation for being unable to pay helps in making the distinction between true zeros and protesters. Thus, the conservative strategy may be excluding those who object to pay from the analysis However, those who object to pay are not necessarily 'protests', they are just more likely to be 'protests'. Thus, further research should be focused on deriving the propensity of 'protest' depending on the stated motivation for being unwilling to pay.

With regards to the policy implications of the analysis of stated willingness to pay, we argue that the current situation with unregulated out-of-pocket payments for physician services represent a substantial financial burden to the public in Central and Eastern European countries, especially in Romania and Ukraine, where informal charges are widely spread, and to some extent in Lithuania. Measures to formalize health expenditure should become a policy priority in the Central and Eastern European region in general. Informal and quasi-formal 
payments are non-transparent and do not account for patients socio-economic status, while wisely designed official patient payments would.

We argue that under certain conditions patient payments for physician services are an acceptable policy tool in Central and Eastern European countries. This conclusion is done based on the observations of rather high shares of the populations that are willing to pay, and levels of willingness to pay are comparable to the service cost or even cover them. The preconditions for the implementation of official charges include feasible payment levels, effective exemption criteria, and eradication of informal charges, as well as the provision of services with good quality and quick access. Small co-payment levels would not cause too many drop-outs from the potential demand pools in all the six Central and Eastern European countries in the study, but the exact levels should be studied with the country-specific instruments. The vulnerable groups subject to fee exemption might be based on household income level in Bulgaria, Lithuania, Hungary, Poland, and Romania.

In Ukraine, age should also be seen as an exemption criterion in addition to income. Strategies to impose penalizing fees for direct referrals should be considered in Ukraine, as it is in most Central and Eastern European countries. Informal charges might become a challenge for the introduction of official patient charges due to no preference for paying formally. However, there is no explicit preference for paying informally, which calls for active measures to build social resistance towards informal payments.

It is also discussed that willingness to pay for physician services is driven by the quality level of the services. In the Ukrainian example, it is shown that benefits gained from quality improvements are rather high and are comparable to service cost. Therefore, any patient payment policy should be tied to quality and access improvements. We also find that, apart from clinical quality and safety, patients value most the interpersonal aspects of care (attitude, information, involvement etc.), especially the elderly people. Therefore, investments in quality enhancement programs should be targeted at the training and education of medical staff, especially in the behavioral aspects, rather than at infrastructure.

In our study, we provide an example of how willingness to pay derived from stated preferences might be used in the assessment of patient charges and their design. Despite their limitations, stated preference techniques provide better and more information than national referendums on the acceptability of patient payments. Discrete choice experiments provide ordinal measures that are useful in setting priorities for investments in quality characteristics 
of the health benefits. Contingent valuation values of willingness to pay provide holistic measures which may be used as an indication of service value.

Thus, stated preferences may be used to assess the level of acceptability of official patient payments for physician services, defining feasible levels of payments and their heterogeneity across population groups, and for defining investment priorities in the quality and access characteristics of the services. Gradual implementation with constant reassessment will help to arrive at optimal policies which best address policy objectives, do not impair equity and access, and match public preferences.

Our study demonstrates the way stated preference techniques may be used to study potential effects of patient charges on health care consumption in the non-existent markets. Patient charges for basic physician services, though ignored and dreaded in Central and Eastern European countries, may be an efficient policy tool in light that people in these countries are ready and able to pay substantial amounts formally if the services are provided with adequate quality and quick access. The assurance of services of good quality and quick access, as well as the elimination of informal payments will be an essential step prior to the implementation of such charges. 



\section{Samenvatting}

\section{De bereidheid tot het betalen voor diensten van artsen in Oekraïne en andere Centraal en Oost-Europese landen: een toepassing van stated-preferences technieken om patiëntenvoorkeuren te meten.}

Om de baten van de gezondheidszorg te meten wordt er in toenemende mate gebruik gemaakt van de zogenaamde stated-preference techniek; een waarderingsmethode waarbij de betalingsbereidheid voorspeld wordt aan de hand van hypothetische situaties en scenario's. De stated-preferences hebben een aantal voordelen zoals de mogelijkheid een waarde toe te kennen aan niet-markt goederen en diensten en de waarde mee te rekenen van passieve gebruikers, niet-gebruikers en potentiële gebruikers. Desalniettemin spelen stated-preference technieken een minimale rol in het vaststellen van eigen bijdragen en het ontwerp daarvan. Dit komt door de onzekere voorspellende, theoretische en convergente validiteit van deze technieken. Echter, in veel gezondheidszorgsystemen waar gezondheidsbaten nooit vermarkt zijn geweest, zoals in veel postsocialistische Centraal en Oost-Europese landen, is het gebruik van stated-preference technieken de enige manier om de betalingsbereidheid te beoordelen.

Eigen bijdragen van patiënten voor de diensten van artsen is een efficiënte manier om onnodig gebruik van deze diensten te reduceren en kan de informele betalingen (die wijdverspreid zijn in Centraal- en Oost-Europa) vervangen en tot op zekere hoogte gezien worden als een aanvullende bron van financiering. Desondanks zijn in Centraal- en OostEuropa in de postsocialistische context veel landen niet bereid een dergelijke impopulaire maatregel te nemen, al is er geen onderzoek gedaan naar eigen bijdragen en de effecten daarvan. Veel bewijsmateriaal is gebaseerd op revealed-preference data (i.e. data betreffende het gebruik van diensten in het verleden) en komt uit West Europa, de Verenigde Staten en enkele Aziatische landen, waar eigen bijdragen al effectief geïmplementeerd zijn. Voor de Centraal en Oost-Europese landen ontbreekt dergelijk bewijs.

Zoals beschreven in hoofdstuk $\mathbf{1}$ is de algemene doelstelling van deze dissertatie het toepassen van stated-preference technieken om de betalingsbereidheid voor dienstverrichtingen van artsen te bestuderen in Centraal en Oost-Europese landen. Daarnaast wordt geanalyseerd tot op welke hoogte de schattingen van betalingsbereidheid kunnen worden gebruikt in het vaststellen van eigen bijdragen voor diensten van artsen. De landen 
Bulgarije, Hongarije, Litouwen, Polen, Roemenië en Oekraïne worden in deze dissertatie behandeld. Deze landen bieden een relevante context voor een cross-country vergelijking gezien zowel de overeenkomsten in de zorgsector tijdens de communistische periode, als de verschillen in tempo waarmee hervormingen in de zorgsector tijdens de postcommunistische periode zijn doorgevoerd.

In alle bovengenoemde landen, behalve Oekraïne, zijn sociale zorgverzekeringen geïntroduceerd. In Oekraïne is nog steeds sprake van een publiek gefinancierd systeem (het USSR Semashko systeem). In de meeste van deze landen betalen patiënten voor bepaalde artikelen (zoals farmaceutica en hulpmiddelen). Echter, betalingen voor basis gezondheidszorgdiensten zijn niet alledaags. Alleen in Bulgarije is in 2000 een relatief lage vergoeding van $€ 1$ voor een bezoek aan een arts geïntroduceerd. In Hongarije werd iets dergelijks in 2007 ingevoerd, maar in 2008 weer afgeschaft na een nationaal referendum. In de andere vier landen zijn formele betalingen door de patiënt (in de tijd dat deze dissertatie is geschreven) alleen gebruikelijk voor diensten die buiten het basis zorgpakket vallen. In alle landen echter is er sprake van quasi-formele betalingen (die vastgesteld worden door zorgverleners in de afwezigheid van nationale regelgeving), en informele (zwarte) betalingen op alle niveaus van de zorgverlening, inclusief voor de dienstverrichtingen van artsen.

In hoofdstuk 2 worden de publieke voorkeuren voor de kwaliteits- en toegangskenmerken van poliklinische diensten in Oekraïne in relatieve en monetaire termen verklaard. De methode van het discrete keuze experiment wordt gebruikt in een steekproef van 303 respondenten die representatief zijn voor de Oekraïense bevolking. Binnen de steekproef is er geen duidelijke voorkeur voor formele dan wel informele betalingen. We vinden ook geen bewijs dat een bezoek aan een huisarts te prefereren is boven directe toegang tot een medische specialist. Er zijn echter wel verschillen tussen bevolkingsgroepen. Kwaliteit-gerelateerde eigenschappen betreffende de dienstverlening van artsen schijnen voor de respondenten belangrijk te zijn, met name de houding van de medische staf. Het verbeteren van de interpersonele aspecten in de poliklinische zorg - door te investeren in training van de medische staf - moet dus prioriteit krijgen wanneer men moet beslissen over investeringen in kwaliteitsverbeteringen in Oekraïne. Andere aspecten zoals sociale kwaliteit en toegang tot zorg, zijn ook belangrijk, maar een verbetering daarvan geeft minder sociale winst.

Hoofdstuk 3 richt zicht op het potentieel en de haalbaarheid van het invoeren van officiële eigen bijdragen voor publieke gezondheidszorgdiensten in Oekraïne. Hiervoor worden patronen van de aanvaardbaarheid van vergoedingen, evenals het vermogen en de 
bereidwilligheid te betalen voor de publieke gezondheidszorg onder bevolkingsgroepen bestudeerd. Hierbij wordt gebruik gemaakt van contingente waarderingsdata die verzameld zijn onder 303 respondenten die representatief zijn voor de Oekraïense volwassen bevolking (dezelfde als die in hoofdstuk 2). Drie beslispunten werden gedefinieerd: het bezwaar tegen de betaling, het onvermogen om te betalen en het niveau van een positieve bereidheid om te betalen. De waarschijnlijkheid van bezwaar tegen betaling wordt grotendeels bepaald door de kwaliteitskenmerken van de diensten. Bezwaar tegen betaling is niet gerelateerd aan dergelijk gedrag in de werkelijkheid. De waarschijnlijkheid om niet te kunnen betalen hangt samen met een hogere leeftijd, een groter aandeel niet-verdienende leden in het huishouden en een lager inkomen. Het niveau van een positieve bereidheid om te betalen is positief gerelateerd aan het inkomen (+7\% per 1000 UAH inkomensverhoging) en is lager voor mensen die een arts hebben bezocht en niet hebben betaald (-22\%). De enigszins substantiële bereidheid tot betaling (tussen $0.9 \%$ en $1.9 \%$ van het huishoudelijk inkomen) voor één bezoek aan een arts, wijzst op een potentieel voor officiële eigen bijdragen in Oekraïne. De patronen van onmogelijkheid tot betalen ondersteunen de behoefte aan uitzonderingscriteria gebaseerd op leeftijd, inkomen, en economische status. De patronen van de bereidheid tot betalen benadrukken de noodzakelijkheid voor betalingen al naar gelang het inkomen.

Hoofdstuk 4 beoogt de geschatte bereidheid tot het betalen voor dienstverrichtingen van artsen verkregen door het discrete keuze experiment en door de contingente waarderingsmethode (de twee studies gepresenteerd in hoofdstuk 2 en 3, gebaseerd op één meting) met elkaar te vergelijken. Er wordt gekeken naar de consistentie tussen de schattingen en of er systematische verschillen zijn tussen de twee typen schattingen. Het discrete keuze experiment geeft een hogere schatting van betalingsbereidheid dan de contingente waarderingsmethode, en de schattingen zijn niet consistent tussen de twee technieken.

Het verschil tussen de schattingen van betalingsbereidheid verkregen door het discrete keuze experiment en deze verkregen door de contingente waarderingsmethode is hoger (i) voor respondenten die zich niet onderscheiden in de verschillende profielen, en (ii) voor een toename in de gepresenteerde niveauveranderingen van de eigenschappen in de profielen. De implicaties van het bereiken van een betere convergentie tussen de schattingen verkregen door de twee verschillende technieken, worden besproken.

In hoofdstuk 5 geven we bewijs betreffende de potentiële impact van eigen bijdragen op het gebruik van gespecialiseerde diensten van artsen in de zes Centraal en Oost-Europese landen die onderdeel zijn van deze studie. We passen een semi-parametrische 'survival' analyse toe 


\section{Samenvatting}

op de gestelde betalingsbereidheid en de mogelijkheid tot betalen om zodoende de potentiële 'pools van vraag' te identificeren, i.e. het aandeel van de bevolking dat bereid en in staat is een bepaalde vergoeding te betalen als er behoefte is aan een medische dienstverrichting. We berekenen ook prijs, inkomen en leeftijd semi-elasticiteit. Data zijn verzameld door middel van nationale enquêtes gehouden in 2010 onder een representatieve steekproef van ongeveer 1000 respondenten in ieder van de zes Centraal en Oost-Europese landen die deel uitmaken van de analyse. Onze resultaten suggereren dat de mediaan van de bereidheid en mogelijkheid tot betalen in de bestudeerde landen varieert van $€ 5.15$ tot $€ 12.2$, en de ranking van landen naar bereidheid en mogelijkheid tot betalen volgt precies de ranking naar inkomensniveau. Lage vergoedingen voor diensten, tot $€ 2.5$ in Bulgarije, Hongarije, Litouwen en Roemenië, en tot $€ 5$ in Polen, zullen niet veel mensen uit de pool van vraag verjagen. Het lagere betalingsinterval moet in meer detail bestudeerd worden voor Oekraïne, waarbij gebruik gemaakt moet worden van een gedetailleerdere schaal. Officiële betalingen voor diensten mét vrijstelling- of lagere eigen bijdragen voor bepaalde groepen wordt als een aantrekkelijk alternatief gezien voor informele betalingen.

Hoofdstuk 6 wordt gewijd aan een beoordeling van de asymmetrische betalingsbereidheid voor gespecialiseerde dienstverrichtingen door artsen. De analyse is gebaseerd op dezelfde data als in hoofdstuk 5, i.e. contingente waarderingsdata van zes Centraal en Oost-Europese landen: Bulgarije, Hongarije, Litouwen, Polen, Roemenië en Oekraïne. De niveaus van betalingsbereidheid worden gemodelleerd met een stochastic frontier regressie, met positieve scheefheid (overstatement) en negatieve scheefheid (understatement). De niveaus van betalingsbereidheid worden gekalibreerd, gebaseerd op individuele voorspellingen van de inefficiëntiescores.

De onbewerkte en gekalibreerde niveaus van betalingsbereidheid worden vergeleken met de daadwerkelijke uitgaven. Onze resultaten suggereren dat de scheefheid van de betalingsbereidheid dwars door de landen heen wordt verklaard door de interactie van drie hoofdfactoren: de waargenomen impact op het actuele beleid, de relevantie en wenselijkheid van de gewaardeerde veranderingen, en ervaringen met betalingen in het verleden. In landen waar verplichte betalingen effectief zijn en ten gevolge daarvan prijsverhogingen aannemelijk zijn, zouden respondenten hun werkelijke betalingsbereidheid verborgen kunnen houden. Daartegenover staan de landen waar prijsverhogingen niet waarschijnlijk zijn en waar de wens voor verbeteringen kan leiden tot een overstatement van de werkelijke betalingsbereidheid. Ongeacht de aangenomen schatting, i.e. onbewerkt of gekalibreerd, 
spenderen Oekraïners en Roemenen meer aan medische dienstenverrichtingen dan hun maximale betalingsbereidheid voor snel toegankelijke diensten van hoge kwaliteit, wat aangeeft dat de huidige ongereguleerde betalingen een substantiële drempel opwerpen in deze landen.

Hoofdstuk 7 presenteert de discussie van de belangrijkste bevindingen uit hoofdstuk 2 t/m 6 . Ze worden verdeeld in twee delen: bevindingen gerelateerd aan het gebruik van statedpreferences om eigen bijdragen te beoordelen, en gerelateerd aan the beleidsimplicaties voor het ontwerpen van eigen bijdragen in de zes Centraal en Oost-Europese landen. We beargumenteren dat de consistentie tussen het discrete keuze experiment en de contingente waarderingsmethode toeneemt met het belang dat de respondenten aan een gepresenteerde taak hechten. We bespreken ook dat de gestelde betalingsbereidheid wordt beïnvloed door zowel de waargenomen consequenties voor toekomstig beleid als door ervaringen met betalingen en het gebruik van zorg in het verleden. Onze bevindingen geven ook aan dat er verschillende mechanismes ten grondslag liggen aan de beslissing om te willen betalen en het niveau daarvan. Vanuit een beleidsperspectief bespreken we dat huidige ongereguleerde betalingen uit eigen zak voor dienstverrichtingen door artsen een substantiële drempel vormen voor patiënten in Centraal en Oost-Europese landen. We betogen dat onder bepaalde voorwaarden eigen bijdragen een acceptabele beleidstool is in Centraal en Oost-Europese landen. Tot slot geven we aan dat mensen in Centraal en Oost-Europese landen bereid zijn te betalen voor kwaliteit en toegang, en beleid over eigen bijdragen zal gebaseerd moeten worden aan de verbetering hiervan.

Ter conclusie geven we een voorbeeld hoe betalingsbereidheid afgeleid van statedpreferences gebruikt kan worden in de raming van eigen bijdragen voor dienstverrichtingen door artsen en het ontwerp daarvan: het niveau van aanvaardbaarheid van deze betalingen, het definiëren van haalbare niveaus van betalingen en de heterogeniteit onder bevolkingsgroepen, en voor de definiërende investeringsprioriteiten in de kwaliteits- en de toegangskarakteristieken van de dienstverrichtingen. Eigen bijdragen voor basiszorg, hoewel vermeden en gevreesd in Centraal en Oost-Europese landen, kunnen een efficiënte beleidsinstrument zijn in de zin van dat mensen in deze landen er klaar voor, en in staat toe, zijn formele substantiële bedragen te betalen als de diensten makkelijk toegankelijk en van hoge kwaliteit zijn. De zekerheid van diensten van goede kwaliteit en makkelijke toegankelijkheid, evenals de eliminering van informele betalingen zijn een essentiële voorwaarde voor het implementeren van eigen bijdragen. 



\section{Анотація}

Готовність сплачувати за послуги лікарів в Україні та інших країнах Центральної та Східної Свропи: Застосування висловлених уподобань для оцінювання платежів паціснтів.

Готовність сплачувати, отримана із застосуванням методів висловлених уподобань (методів оцінювання певних благ у гіпотетичних сценаріях вибору чи купівлі), все частіше використовується для оцінювання послуг охорони здоров'я. Висловлені вподобання мають низку переваг, зокрема вони дозволяють отримати оцінки товарів та послуг, що раніше були відсутні на ринку, а також отримати оцінки від пасивних i потенційних споживачів, а також тих, хто не користувався цими послугами. Однак, ці методи відіграють досить обмежену роль в оцінюванні платежів пацієнтів та плануванні їхнього дизайну. Це відбувається через невизначену достовірність та надійність оцінок, отриманих за допомогою цих методів. 3 іншого боку, в багатьох системах охорони здоров'я, де послуги ніколи не були предметом торгівлі, як, зокрема, у багатьох пост-соціалістичних країнах Центральної та Східної Європи, висловлені вподобання є єдиним можливим методом оцінювання готовності сплачувати.

Платежі пацієнтів є ефективним інтрументом політики в охороні здоров'я, здатним зменшити надмірне споживання послуг, врегулювати неофіційні платежі (дуже поширені у країнах Центральної та Східної Свропи), а також до певної міри генерувати додаткові кошти. В Центральній та Східній Свропі, однак, в пост-соціалістичному контексті, уряди більшості країн не готові впроваджувати такі непопулярні заходи, як офіційні платежі пацієнтів. Однак, наукових підстав для такої відмови практично немає. Більшість проведених досліджень базуються на виявлених вподобаннях (даних фактичного споживання), а тому походять із країн Західної Свропи, США, та деяких розвинених країн Азії, де платежі пацієнтів вже ефективно впроваджені. В країнах Центральної та Східної Європи таких досліджень не проводилося.

Як окреслено в розділі 1, загальна мета цієї дисертації - застосувати методи висловлених вподобань для оцінювання готовності сплачувати за послуги лікарів в країнах Центральної та Східної Свропи, а також проаналізувати наскільки готовність сплачувати, отримана 3 їх використанням, може бути застосована в оцінюванні 


\section{Анотація}

політики платежів пацієнтів за послуги лікарів. До цього дослідження увійшли шість країн: Болгарія, Угорщина, Литва, Польща, Румунія та Україна. Ці країни мали подібні системи охорони здоров'я в комуністичні часи, але різні траєкторії та темпи реформ у пост-комуністичний період, а тому є хорошою базою для порівняння.

В усіх країнах-учасницях цього дослідження, окрім України, було впроваджено системи соціального страхування. В Україні ж і до сьогодні діє система охорони здоров'я, фнінасована із державного бюджету (система Семашко як у СРСР). В більшості $з$ цих країн існують платежі пацієнтів за медичні товари (препарати та обладнання). Однак, платежі пацієнтів за базові послуги охорони здоров’я існують лише в Болгарії. Там, відносно невисокий платіж (близько € 1 за візит до лікаря) було впроваджено у 2000 р. В Угорщині такий невисокий платіж (близько €1 за візит) проіснував один рік у 2007 р., але був скасований за результатами всенародного референдуму у 2008 р. В інших чотирьох країнах офіційна оплата на час проведення дослідження існувала лише за послуги поза страховим пакетом. В усіх цих країнах, однак, квазі-офіційні платежі, встановлені надавачами послуг охорони здоров'я за відсутності державного регулювання, а також неофіційні платежі існують на всіх рівнях надання допомоги, включно з послугами лікарів.

У розділі 2 цієї дисертації, досліджено громадські вподобання щодо характеристик якості та доступу послуг лікарів в Україні у відносному та монетарному виразі. Для цього застосовано експеримент дискретного вибору на вибірці із 303 респондентів, що репрезентують доросле населення країни. На рівні вибірки не спостерігаємо вираженої переваги сплачувати офіційно, чи навпаки - неофіційно. Також немає чітких вподобань щодо того, відвідувати лікаря загальної практики (дільничного лікаря) чи звертатися одразу ж до вузького спеціаліста. Однак, є певна неоднорідність цих уподобань серед соціально-демографічних груп населення. Важливими при виборі лікаря є атрибути якості послуг охорони здоров'я, особливо ставлення медичного персоналу. Отже, покращення міжососбистісних аспектів первинної допомоги шляхом інвестицій в освіту та навчання медичного персоналу має стати пріоритетом у фінансуванні охорони здоров’я. Інші аспекти якості та доступу також важливі для пацієнтів, однак їхнє покращення принесе менше вигоди.

Розділ 3 зосереджений на потенціалі та можливості впровадження офіційних платежів пацієнтів за послуги лікарів шляхом вивчення їхньої прийнятності, а також здатності та готовності сплачувати за ці послуги серед груп населення. Для цього використано дані 
умовного оцінювання проведеного серед 303 респондентів, що реперзентують доросле населення країни (як у розділі 2). Виокремлено три рівні прийняття рішення: відмова сплачувати, неспроможність сплачувати, та рівень готовності сплачувати. Імовірність відмови сплачувати найбільше пов'язана 3 якісними характеристиками послуги. Відмова сплачувати в гіпотетичному завданні не пов'язана із відповідною поведінкою на реальному ринку. Імовірність бути неспроможним/ою сплачувати пов'язана зі старшим віком та нижчим економічним статусом. Рівень готовності сплачувати позитивно пов'язаний із доходом, а також є нижчим серед людей, які відвідували лікарів але не платили за це. Досить високий рівень готовності сплачувати (між 0.9\% та 1.9\% доходу домогосподарства) за візит до лікаря вказує на потенціал офіційних платежів в Україні. Картина неспроможності сплачувати вказує на необхідність критеріїв виключення, що базуватимуться на віку, доході, та інших аспектах економічного статусу. Рівні готовності сплачувати підкреслюють, що платежі повинні бути пропорційні до доходу.

Розділ 4 має на меті дезагреговане порівняння оцінок готовності сплачувати, отрманих iз експерименту дискретного вибору та умовного оцінювання (двох методів, застосованих у розділах 2 та 3 на єдиній вибірці). Для цього досліджено узгодженість між цими оцінками, а також чи є систематична різниця між ними. За нашими результатами, оцінки, отримані з експерименту дискретного вибору, є вищими, ніж 3 умовного оцінювання, але вони неузгоджені між собою. Різниця між цими оцінками росте (i) для респондентів, які не розрізняють представлені якісні профілі послуг, та (ii) зі зростанням різниці у рівнях якісних атрибутів між представленими до вибору варіантами. Наслідки цих результатів для досягнення кращої узгодженості між оцінками готовності сплачувати обговорено в цьому розділі.

У розділі 5 оцінено можливий вплив платежів пацієнтів на споживання послуг медичних спеціалістів в шести країнах Центральної та Східної Свропи, що увійшли в це дослідження: Болгарії, Угорщині, Литві, Польщі, Румунії та Україні. Для цього застосовано напівпараметричний аналіз виживання до даних із готовності та здатності сплачувати за ці послуги. Це дозволило побудувати криві потенційного попиту, що показують частки населення, яке готове та спроможне сплачувати платежі певного рівня в разі медичної потреби. Також обраховано цінові еластичності, а також еластичності за доходом та віком. Дані було зібрано за допомогою опитувань, провдених у 2010p. серед реперезентативних вибірок в шести країнах, близько 1000 


\section{Анотація}

респондентів у кожній із країн. Результати цього дослідження свідчать, що медіанна готовність та можливість сплачувати коливається від $€ 5.15$ до $€ 12.2$, а порядок країн за цим показником відповідає рівню доходів в країні. Невисокі платежі, до $€ 2.5$ в Болгарії, Угорщині, Литві та Румунії, та до $€ 5$ в Польщі не зумовлюють суттєвого зниження потенційного попиту. Нижній ціновий інтервал необхідно детальніше вивчати в Україні із застосуванням шкали, чутливої до менших змін готовності сплачувати. В цьому розділі аргументовано, що платежі пацієнтів можуть бути виграшною альтернативою неофіційним платежам в разі впровадження відповідних критеріїв виключення та пільг.

Розділ 6 присвячений належному оцінюванню скошених даних готовності сплачувати за послуги медичних спеціалістів. Аналіз базується на тих же даних, що й у розділі 5, тобто даних умовного оцінювання із шести країн Центральної та Східної Європи: Болгарії, Угорщини, Литви, Польщі, Румунії та України. Рівень готовності сплачувати оцінено із застосуванням моделі стохастичного кордону, враховуючи позитивну та негативну скошеність даних, яка трактується як відповідно завищування та занижування справжньої готовності сплачувати частиною респондентів. Рівень готовності сплачувати зважено на предбачені коефіцієнти неефективності (завищування чи занижування). Незважені та зважені оцінки порівняно із фактичними річними видатками на послуги медичних спеціалістів. Результати цього розділу свідчать, що напрям скошеності готовності сплачувати, тобто занижування чи завищування оцінок, в різних країнах пояснюється спільним впливом таких факторів: очікуваним впливом результатів опитування на реальну цінову політику, актуальності та бажаності оцінюваних якісних змін, і частотою споживання послуг. В країнах, де платежі пацієнтів діють і, відповідно, можливим є підняття цін, респонденти, особливо споживачі послуг, схильні приховувати справжній рівень готовності сплачувати. В країнах, де впровадження чи підняття цін $є$ малоймовірним, бажаність змін може призвести до завищування справжньої готвність сплачувати, особливо серед нечастих користувачів.

У Розділі 7 представлено обговорення основних результатів, описаних в попередніх розділах. Їх розділено на дві групи: ті, що стосуються використання висловлених вподобань для оцінки платежів пацієнтів, а також ті, що стосуються висновків для політики платежів пацієнтів в шести країнах Центральної та Східної Свропи. Ми доводимо, що узгодженість між оцінками готовності сплачувати, отриманими із 
екперименту дискретного вибору та умовного оцінювання, зростає зі зростанням важливості атрибутів та послуг представлених респонденту. Також ми показуємо, що рівень висловленої готовності сплачувати залежить від очікуваних наслідків опитування для політики платежів, необхідності якісних покращень, та частоти споживання. Також наші результати дозволяють говорити про те, що різні механізми лежать в основі рішення бути готовим сплачувати та визначення рівня готовності сплачувати. 3 точки зору політики, ми показуємо що теперішня ситуація 3 неврегульованими платежами $є$ суттєвим фінансовим тягарем для пацієнтів в Центральній та Східній Європі. Ми також доводимо, що за певних умов платежі пацієнтів є прийнятним інструментом політики в охороні здоров'я в цих країнах. Насамкінець, показано, що люди в країнах Центральної та Східної Свропи готові сплачувати за якість та доступ, а тому будь-які впровадження платежів повинні супроводжуватися покращенням цих характеристик.

Отже, в цій дисертації показано як готовність сплачувати, оцінена за допомогою висловлених уподобань, може бути використана для оцінювання платежів пацієнтів та аспектів їхнього дизайну: прийнятності таких платежів, визначенні рівня платежів, неоднорідності готовності сплачувати серед груп населення, визначення інвестиційних пріоритетів в якість та доступ. Платежі пацієнтів за послуги охорони здоров'я, хоча їх i уникають в країнах Центральної та Східної Європи, можуть стати ефективним інструментом політики в тому сенсі, що люди готові офіційно сплачувати суттєві кошти за послуги адекватної якості та доступу. Забезпечення такої якості і доступу, а також викорінення неофіційних платежів, буде необхідним кроком у встановлені оптимальної політики платежів пацієнтів. 



\section{Аннотация}

Готовность платить за услуги врачей в Украине и других странах Центральной и Восточной Европы: Применение выраженных преференций для оценки платежей пациентов.

Готовность платить оцененая с помощью методов выраженных предпочтений (методов оценки благ в гипотетических сценариях выбора или покупки) все чаще используется для оценивания услуг здравоохранения. Выраженные предпочтения имеют ряд преимуществ, в частности они позволяют получить оценки товаров и услуг, которых раньше на рынке не было, а также получить оценки пассивных и потенциальных пользователей этих услуг, и тех, кто не пользовался ими. Однако, эти методы играют ограниченную роль в оценке платежей пациентов и планировании их дизайна. Это просиходит по причине неопределенной достоверности и надежности оценок, полученых с помощью этих методов. С другой стороны, во многих системах здравоохранения, где услуги никогда не были предметом торговли, как, в частности, во многих пост-социалистических странах Ценральной и Восточной Европы, выраженные предпочтения являются единственным способом оценки готовности платить.

Платежи пациентов являются эффективным инструментом полити здравоохранения, который способен уменьшить чрезмерное использование услуг, уреглировать неофициальный рынок (очень распостраненный в странах Ценральной и Восточной Европы), а также в некоторой степени генерировать дополнительные стредства. В Ценральной и Восточной Европе, однако, в пост-социалистическом контексте, правительства большинства стран не готовы внедрять такие непопулярные меры, как официальные платежи пациентов. Научных обоснований для такого отказа практически нет. Большинство проведенных исследований базируются на выявленных предпочтениях (данных фактического использования), и поэтому были проведены в странах Западной Европы, США и некоторых развитых странах Азии, где платежи пациентов уже эффективно внедрены.

Как описано в разделе 1, общая цель этой дисертации - применить методы выраженных предпочтений для оценки готовности платить за услуги врачей в странах Центральной и Восточной Европы, а также проанализировать насколько готовность 
платить, полученная с примнением єтих методов, может быть использована для оценивания политики платежей пациентов за услуги врачей. В данное исследование вошли шесть стран: Болгария, Венгрия, Литва, Польша, Румыния и Украина. Эти страны имели похожие системы здравоохранения в коммунистические времена, но разные траектории и темпы реформ в пост-коммунистический период, а поэтому являют собой хорошую базу сравнения.

Во всех странах-учасницах этого исследования, кроме Украины, были внедрены системы социального страхования. В Украине же и по сей день действует система здравоохранения, финансированная из государственного бюджета (система Семашко как в СССР). В большинстве этих стран действуют платежи пациентов за медицинские товары (препарты и оборудование). Однако, платежи пациентов за базовые услуги здравоохранения действуют только в Болгарии. Там относительно невысокий платеж (около $€ 1$ за визит к врачу) был утсановлен в 2000 г. В Венгрии такой же небольшой платеж (около $€ 1$ за визит к врачу) просуществовал всего один год в 2007 г., но был отменен на всенародном реферндуме в 2008 г. В остальных четырех странах официальная оплата на время проведения исследования взималась только за услуги вне страхового пакета. Во всех этих странах, однако, квази-официальные плетжи, установленные провайдерами услуг из-за отсутсвия государственного регулирования, а также неофициальные платежи существуют на всех уровнях оказания помощи, включительно с услугами врачей.

В разделе 2 этой диссертации, исследованы общественные предпочтения касательно характерстик качества и доступа услуг врачей в Украине в относительном и монетарном выражении. Для этого применено эксперимент дискретного выбора на выборке из 303 респондентов, репрезентирующих взрослое население страны. На уровне выборки не наблюдается четко выраженных предпочтений платить официально, или наоборот - неофициально. Также нету четких предпочтений осуществлять визит к врачу общей практики (участковому врачу), либо обращаться напрямую к узкому специалисту. Однако, есть некоторая неоднородность этих предпочтений среди групп населения. Важными при выборе врача являются атрибуты качества услуг здравоохранения, особенно отношение медицинского персонала. Следовательно, улучшение межличностных аспектов первичной помощи путем инвестиций в образование и обучение медицинского пресонала должны стать приоритетом 
финансирования здравоохранения. Другие аспекты качества и доступа также важны для пациентов, но их улучшение принесет меньше выгоды обществу.

В разделе 3 рассматривается потенциал и возможности внедрения официальных платежей пациентов за услуги врачей путем исследования их приемлимости, а также способности и готовности платить за эти услуги среди групп насления. Для этого использованы данные условного оценивания проведенного среди 303 респондентов, которые репрезентируют взрослое население Уркаины (как и в разделе 2). Выделено три уровня принятия решений: отказ платить, неспособность платить, и уровень готовности платить. Вероятность отказа платить более всего связана с качестовм услуг. Отказ платить в гипотетичском задании не связан с соответствующим поведением в реальной жизни. Вероятность быть неспособным/ой платить связана со старшим возрастом и худшим экономическим статусом. Уровень готовонсти платить позитивно связан с доходом, а также является ниже среди людей, которые обращались к врачам но не платили за это. Довольно высокий уровень готовоности платить (между 0.9\% и 1.9\% от дохода домохозяйства) за визит к врачу указывает потенциал официальных платежей в Украине. Картина неспособности платить указывает на необходимость критериев исключения, котрые бы базировались на возрасте, дохде, а также других аспектах экономического статуса. Уровни готовности платить подчеркивают, что платежи пациентов должны быть пропорциональны доходу.

Раздел 4 имеет целью дезагрегированное сравнение оценок готовности платить, полученых с помощью експереимента дискретного выбора и условного оценивания (двух методов, примененных в разделах 2 и 3 на единой выборке). Для этого изучена согласованность между этими оценками, а также есть ли систематическая разница между ними. По нашим результатам, оценки эксперимента дискретного выбора выше нежели условного оценивания, но они не согласованны между собой. Разница между оценками выше (i) для респондентов, котрые не отличают представленые качественные профили услуг, и (ii) при росте разницы в уровнях качественных атрибутов между представлеными для выбора вариантами. Выводы из таких результатов для достижения лучшей согласованности между оценками готовности платить обсуждены в этом разделе.

В разделе 5 оценено возможное влияние платежей пациентов на потребление услуг медицинских специалистов в шести странах Центральной и Восточной Европы, которые вошли в исследование: Болгарии, Венгрии, Литве, Польше, Румынии и 
Украине. Для этого применен полупарметрический анализ выживаемости на данных про готовность и способность платить за эти услуги. Это позволило построить кривые потенциального спроса, котрые показывают доли населения, готовых и способных платить платежи разного уровня при медицинской необходимости. Также были посчитаны ценовые эластичности, а также эластичности по доходу и возрасту. Данные были получены с помощью опросов, проведеных в 2010г. среди репрезентативных выборок в шести странах, около 1000 респондентов в каждой из стран. Результаты этого исследования показывают, що медианная готовность платить варьируется от $€ 5.15$ до $€ 12.2$, а порядок стран за этим показателем соответсвует уровням дохода в них. Невысокие платежи до $€ 2.5$ в Болгарии, Венгрии, Литве и Румынии, и до $€ 5$ в Польше не вызовут существенного снижения потенциального спроса. Нижний ценовой интервал необходимо подробнее изучать в Украине с использованием шкалы, чувствительной к меньшим изменениям готовности платить. В этом разделе аргументировано, что платежи пациентов могут быть лучшей альтернативой неофициальным платежам при внедрении соответсвующих критериев исключения и льгот.

Раздел 6 посвящен надлежащему оцениванию скошенных данных о готовности платить за услуги медицинских специалистов. Анализ основывается на тех же данных, что и в разделе 5 , а именно данных условного оценивания из шести стран Центральной и Восточной Европы: Болгарии, Венгрии, Литвы, Польши, Румынии и Украины. Уровень готовности платить оценен с использованием модели стохастической границы, учитывая позитивную и негативную скошенность данных, которая трактуется как соответсвенно завышение и занижение действительной готовности платить частью респондентов. Уровень готовности платить взвешен на оцененные коэффициенты неэффективности (завышения или занижения). Невзвешенные и взвешенные оценки сравнены с фактическими годовыми издержками на услуги медицинских специалистов. Результаты этого раздела свидетельствуют, что скошенность готовности платить в разных странах объясняется совмесным влиянием следующих факторов: ожидаемым влиянием результатов опроса на реальную политику, актуальности и желаемости оцениваемых изменений, и частотой использования услуг. В странах, где платежи пациентов действительны, и, соответственно, возможным является поднятие цен, респонденты, особенно пользователи услуг, склонны скрывать настоящий уровень готовности платить. В странах, где внедрение или поднятие уровня платежей 
маловероятны, желаемость изменений может вызвать завышение действительной готовности платить, осбенно среди нечастых пользователей медицинских услуг.

В разделе 7 пердставлено обсуждение основных результатов, описаных в предидущих разделах. Их разделено на две группы: те, которые касаются использования выраженных предпочтений для оценки платежей пациентов, а также те, которые касаются выводов для политики платежей в шести странах Центральной и Восточной Европы. Мы показываем что согласованность между оценками готовности платить, полученых с помощью эксперимента дискретного выбора и условного оценивания, растет при увеличении важности атрибутов и услуг, представленных респонденту. Также мы показваем, что уровень выраженной готовности платить зависит от ожидаемых последствий опроса для политики платежей пациентов, необходимости качественных изменений, а также частоты пользования. Также наши результаты позволяют утверждать, что разные механизмы лежат в основе решения быть готовым платить и определением уровня готовности платить. С точки зрения политики, мы показываем, что теперешняя ситуация с неурегулированными платежами является существенным финансовым бременем для пациентов в Центральной и Восточной Европе. Мы также доказываем, что при некторых условиях платежи пациентов являются приемлимым инструментом политики здравоохранения в этих странах. Наконец, показано, что люди в странах Центральной и Восточной Европы готовы платить за качество и доступ, а поэтому любые внедрения политики платежей должны сопровождаться усовершенствованием этих характеристик.

Таким образом, в данном исследовании показано как готовность платить, оцененная с помощью вираженных предпочтений, может быть использована для оценки платежей пациентов и аспектов их дизайна: их приемлемости, определения уровня платежей, неоднородности готовности платить среди групп наседения, определения инвестиционных приоритетов в качество и доступность. Платежи пациентов за услуги здравоохранения, хотя их и избегают в странах Центральной и Восточной Европы, могут стать эффективным инструментом политики в том смысле, что люди готовы официально платить существенные суммы за доступные услуги адекватного качества. Обеспечение такого качества и доступности, а также искоренение неофициальных платежей, будет необходимым шагом при внедрении политики платежей пациентов. 



\section{Thank you words}

This dissertation is a product which was released with the support and compassion of many people. My initiation into the research community happened thanks to dr. Irena Gryga, head of the School of Public Health in Kyiv where I did my master studies. Irena was the person who saw me as a potential researcher and invited to teaching and research team of the School. I was not seriously considering an academic career when Irena offered and convinced me to join the international research project ASSPRO CEE 2007 and follow PhD track within its framework. For this belief, support and lead through the whole process, I want to sincerely thank Irena.

It is not possible to overestimate the contribution and support of my supervisors, dr. Milena Pavlova and prof. dr. Wim Groot. My transformation from a perplexed student with naïve ideas about research to an author of several research papers and a $\mathrm{PhD}$ dissertation became possible thanks to them. I am grateful to Milena for putting enormous amount of effort and time into explaining, edging ideas and reviewing my horrible writings, for correcting and directing them. I suspect that Milena has a time machine; otherwise, it would have been impossible to invest so much work in my writings with all her schedule. Meetings with Wim were always a desired milestone in the progress of this dissertation. Whenever, I was stuck or simply needed advise, I always got what I needed. I express my thanks to Wim for being an excellent supervisor and counselor.

I want to thank for the devoted time to the members of the evaluation committee: prof. dr. Silvia Evers, prof. dr. Igor Burakovsky, prof. dr. Carmen Dirksen, prof. dr. Frits van Merode, and dr. Mychailo Wynnyckyj. I appreciate your input, nice words and critical comments which served for improving this dissertation.

One of the best parts of working on this dissertation is that I met new friends. Juniors of the ASSPRO CEE 2007 project made this work easier and a lot more fun. Elka, Marzena, Petra, Tania, Jelena, Sonila, Vladimir, Reza, it was really good to work and spend time with you. Anil, Param, Florian, despite the fact that you were not in ASSPRO CEE 2007, you became a true part of our team. My special thanks to a great friend and a wonderful person, Martine, who is always willing to help and to have fun. Her contribution to this work is the Dutch translation of the Summary. 
It is easy to get lost in the formalities and the administrative issues, but Suus Koene and Brigitte Caenen never let me do so: many thanks for that. There are many people who contributed to this research by sharing their views and patiently answering the questionnaires. I appreciate your every effort. This research would not be possible without the financial support of the European Commission. I want to thank their representatives who always were interested in this study and gave insightful comments during our presentations.

At the beginning of the $\mathrm{PhD}$ track I had a wonderful opportunity to get training in the Doctoral School of the National University of Kyiv-Mohyla Academy, Ukraine. It was very useful and fascinating. I am especially proud to be part of this School as this is the first European-standard $\mathrm{PhD}$ program in Ukraine. I am grateful to all people involved into establishing the Doctoral School despite the unfriendly Ukrainian environment, especially Mychailo Wynnyckyj, and to our highly professional teachers who gave us an excellent training. I will have many happy memories of the time spend with my friends, first ever Ukrainian PhD-fellows, in and after school.

My family, wife Olya and son Orest, brought me a pleasant and desired distraction from my work, but always supported and understood me when I had to immerse myself back into work. Thank you for being with me! And many thanks to my parents, who were always supportive of my life-choices including my academic track. 


\section{Curriculum Vitae}

Andriy Danyliv was born on 26 July 1984 in Kyiv, Ukraine. He studied economics at the National University of Kyiv-Mohyla Academy in Ukraine and obtained a specialist diploma. For master studies, Andriy changed the focus towards public health and in 2007 obtained a Master of health care management at the School of Public Health, National University of Kyiv-Mohyla Academy, Ukraine (Master program supported by Maastricht University, the Netherlands).

Andriy's research experience started in 2009, when he became a junior researcher and a $\mathrm{PhD}$ student at School of Public Health (Kyiv) in the FP7 Project ASSPRO CEE 2007 funded by European Commission and coordinated by Maastricht University. The focus of the research is the application of the stated preference techniques to elicit willingness to pay for health care services.

Apart from PhD research, Andriy has 6 year experience in teaching Health Economics disciplines and Statistics. He was also involved in the research projects related to costing of the palliative care modes and gender aspects of health care in Ukraine.

At present, Andriy is a health economics postdoctoral researcher at the School of Business and Economics in the National University of Ireland, Galway. His work now is related to exploring different economic issues of the gestational diabetes mellitus and progression to type 2 diabetes, as well as economic effectiveness of the screening and prevention programs.

\section{LIST OF PUBLICATIONS}

\section{International journals}

Danyliv, A., Pavlova, M., Gryga, I., \& Groot, W. (2014). Preferences for physician services in Ukraine: a discrete-choice experiment. The International Journal of Health Planning and Management. Doi: 10.1002/hpm.2239.

Danyliv, A., Pavlova, M., Gryga, I., \& Groot, W. (2013). Willingness to Pay for Physician Services at a Primary Contact in Ukraine: Results of a Contingent Valuation Study. BMC Health Services Research, 13(1), 208. Doi:10.1186/1472-6963-13-208. 


\section{Curriculum vitae}

Danyliv, A., Pavlova, M., Gryga, I., \& Groot, W. (2012). Willingness to pay for physician services: Comparing estimates from a discrete choice experiment and contingent valuation. Society and Economy, 34(2), 339-357. Doi: 10.1556/SocEc.34.2012.2.9.

Danyliv, A., Stepurko, T., Gryga, I., Pavlova, M., \& Groot, W. (2012). Is there a place for the patient in the Ukrainian health care system? Patient payment policies and investment priorities in health care in Ukraine. Society and Economy, 34(2), 273-291. Doi: 10.1556/SocEc.34.2012.2.6.

Gryga, I., Stepurko, T., Danyliv, A., Gryga, M., Lynnyk O., Pavlova, M., \& Groot, W. (2010). Attitudes towards patient payments in Ukraine: is there a place for official patient charges? Zeszyty Naukowe Ochrony Zdrowia. Zdrowie Publiczne I Zarzadzanie, VIII (1), 69-78.

\section{Local Ukrainian journals}

Danyliv, A., Ivanova N., Pavlova, M., \& Groot, W. (2012). Vertical analysis of the demand for medical services in Ukraine. (in Ukrainian) Economic analysis, 10(1), 124-129.

Danyliv, A. (2012). Health care services market formation in Ukraine: structure of financing. (in Ukrainian) Scientific Notes (Науковi записки) of Kyiv-Mohyla Academy. Economics, 133, 49-56.

Danyliv, A., Ivanova, N. (2011). Classification of the methods of estimating demand in health care. (in Ukrainian) Scientific Notes (Науковi записки) of Kyiv-Mohyla Academy. Economics, 120, 102-106.

Danyliv, A.I., Gryga, I.M. (2007). Health care facilities financing: The case of Zolotchiv region in Kharkiv oblast. (in Ukrainian). Health Care in Ukraine. 25(1).

Ivanova N., Danyliv.A. (2006). Evaluation of business Climate of Region: Comparative Analysis of Contemporary Methods. (in Ukrainian) Scientific Notes (Наукові записки) of Kyiv-Mohyla Academy. Economics, 56, 16-22.

\section{Submitted papers}

Danyliv, A., Groot, W., Gryga, I., \& Pavlova, M. Willingness and ability to pay for physician services in six Central and Eastern European countries.

Danyliv, A., Groot, W., Gryga, I., \& Pavlova, M. Calibration of the willingness to pay for physician services in six Central and Eastern European countries: Context implications for the over- and understatement patterns.

Danyliv, A., Gillespie, P., O’Neill, C., Noctor, E, O’Dea, A., Tierney, M., McGuire, B., Glynn, L., \& Dunne, F. Health Related Quality of Life Two to Five Years After Gestational Diabetes Mellitus Within the ATLANTIC DIP Cohort. 